\title{
WestVirginiaUniversity
}

THE RESEARCH REPOSITORY @ WVU

Graduate Theses, Dissertations, and Problem Reports

2004

\section{PIC 18F452 implementation of digital filters}

Vikram A. Bose-Mullick

West Virginia University

Follow this and additional works at: https://researchrepository.wvu.edu/etd

\section{Recommended Citation}

Bose-Mullick, Vikram A., "PIC 18F452 implementation of digital filters" (2004). Graduate Theses, Dissertations, and Problem Reports. 1418.

https://researchrepository.wvu.edu/etd/1418

This Thesis is protected by copyright and/or related rights. It has been brought to you by the The Research Repository @ WVU with permission from the rights-holder(s). You are free to use this Thesis in any way that is permitted by the copyright and related rights legislation that applies to your use. For other uses you must obtain permission from the rights-holder(s) directly, unless additional rights are indicated by a Creative Commons license in the record and/ or on the work itself. This Thesis has been accepted for inclusion in WVU Graduate Theses, Dissertations, and Problem Reports collection by an authorized administrator of The Research Repository @ WVU. For more information, please contact researchrepository@mail.wvu.edu. 
PIC 18F452 IMPLEMENTATION OF DIGITAL FILTERS

\author{
Vikram A Bose-Mullick \\ Thesis Submitted to \\ the college of Engineering \\ at West Virginia University \\ in partial fulfillment of the requirements \\ for the degree of \\ Master of Science \\ in \\ Electrical Engineering
}

Powsiri Klinkhachorn, Ph.D., Committee Chairperson

Roy Nutter, Ph.D.

Robert McConnell, Ph.D.

Lane Department of Computer Science and Electrical Engineering

Morgantown, West Virginia

2004

Keywords: Microchip PIC, 18F452, FIR filter, LMS, Adaptive Filter, Noise Cancellation, Echo Cancellation, Filter Design

Copyright 2004 Vikram A Bose-Mullick 


\title{
ABSTRACT
}

\section{PIC 18f452 implementation of digital filters}

\author{
Vikram A Bose-Mullick
}

This research hopes to explore the computational limits of the PIC18f452 chip by encompassing the designing and implementation of two types of filters for the PIC 18F452 microcontroller. The main purpose of this research is to implement a floating-point least mean square (LMS) error adaptive filter and its secondary goal is a fixed-point implementation of finite impulse response (FIR) filter. FIR filters are specified via a graphical user interface (GUI) and upon demand, optimized C-language code is generated for the popular CCS PIC C-Compiler. In is the intent of this research to learn whether FIR filters can be made computationally viable on the PIC18 chips, can they run stably with reliable and repeatable performance? What is the minimum execution time possible at the processing limits of the chip? And how is filter attenuation affected when taps are scaled down from floating-point to fixed point? For the floating point LMS filter it desired to explore the relationship between sampling-rate and filter order and to develop a hardware optimized floating point library for general use. The minimum execution time for the LMS filter achieved during this research is $26.7 \mu$ s per order. The FIR filter code generation software developed during this study allows graphical specification, inspection of response curves. It ultimately presents three options for automatic code generation - program-space efficient code (uses minimum code space), data-memory efficient code (uses minimum RAM) and speed-efficient code (optimized for quickest execution), thereby allowing up to a 75 th order FIR filter with the best execution time of $800 \mathrm{~ns}$ per MAC cycle achieved at the bit-depth of 8-bit samples and 8-bit taps. The filter tap conversion from floating-point format to 8-bit fixed point reduced the attenuation by an average of $28 \%$. In general, both filters gave a strong performance with consistent, reliable and repeatable results. 


\section{DEDICATION}

Both small and large, to everyone that made a difference. Above all, I dedicate my work to my kind and loving family. 


\section{ACKNOWLEDGEMENT}

I wish to express my deepest possible thanks to Dr Powsiri Klinkhachorn my advisor and dear friend, for his excellent guidance and boundless friendship. Even though I've never received an 'A' from him, I have no regrets because I have learned more in his classes than all my 'A's put together.

A special thanks to Dr. Roy Nutter for bringing microprocessors into my depth of field through his innovative lectures, inspiring chats and for showing us the value of journals and periodicals. They contained the very first sparks that led to my intense love of the subject.

I express my deep appreciation for to Dr. McConnell from whom I learned the fundamentals for analog and digital without which nothing is possible.

Thank you gentleman for your generosity of spirit, for your infinite kindness you're your constant support and finally for making the time to serve in my committee.

A special thanks to my friend Tal Gottesman for filling in and picking up the slack during during zillions of hours that were spent putting this document together.

Finally my thanks to Braxton Lewis and my boss Dr. McCawley for all their constant help and support. 


\section{TABLE OF CONTENTS}

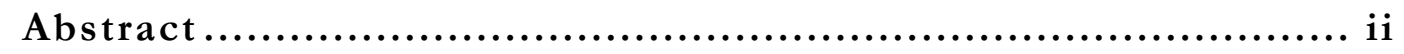

Dedication ........................................................ iii

Acknowledgements .............................................. iv

Table of contents................................................. v

List of Figures................................................ viii

List of Tables ................................................. xii

List of Symbols / Nomenclature .....................................xiii

CHAPTER 1: INTRODUCTION .............................. 1

1.1 Personal Motivation .........................................................................

1.2 Signal Processing using the PIC 18F452 ….............................2

1.3 Digital Filters Vs Analog Filters .....................................................

1.4 PIC 18 Microcontroller Family ………………………………...

1.5 Detailed Research Objectives and Contributions ...............4

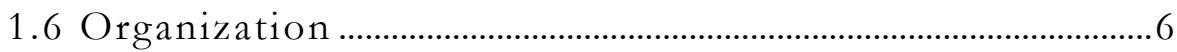

CHAPTER 2: LITERATURE SURVEY .......................... 7

2.1 Classification of Filtering Methods ............................................

2.2 Digital Filters ..................................................................................

2.3 Non-Recursive Type Digital Filters ............................................ 8

2.4 Switching type digital filters ........................................................

2.5 Adaptive Filters ............................................................................

2.6 Least Mean Squared Error (LMS) ……………………………....10

2.8 Implementation of a digital filter ..................................................12 
3.1 Finite Impulse Response (FIR) ..................................................14

3.2 Implementation Background .....................................................15

3.3 FIR Filter Implementation ..........................................................17

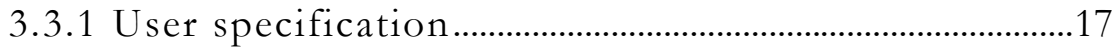

3.3.2 Filter Tap Generation ............................................................20

3.3.3 Coefficient Scaling ..................................................................22

3.3.4 Code generation........................................................................23

3.3.5 Buffering Data.......................................................................24

3.3.6 Sampling: Analog to Digital Conversion ......................35

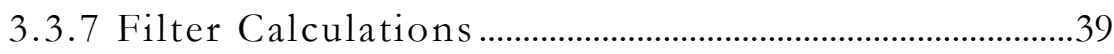

3.3.8 Implementation for Shortest Execution Time ........41

3.3.9 Implementation for Efficient RAM utilization .......51

3.3.10 Implementation for Min Program Memory ..............53

3.4 Implementation of the floating-point LMS filter .............54

3.5 The compilers floating point system ..........................................54

3.6 Floating-Point Word lengths.........................................................55

3.6.1 Algorithm developed for FP multiplication...............57

3.6.2 Calling FP Addition function in C-language ............62

3.6.3 Algorithm developed for floating-point addition ........62

3.6.4 Converting integer to floating-point format ............64

3.7 Implementation of a $4^{\text {th }}$ order LMS algorithm .....................65

3.7.1 Sampling noise and reference for LMS filter ...........66

3.7.2 Program Outline for $4^{\text {th }}$ order LMS filter .....................68

3.8 Hardware Test Circuit ......................................................................73

3.9 Detailed Schematic of the Power Supply ..................................74

3.10 Detailed Schematic: Smoothing Filter.....................................75

3.11 Detailed Schematic: Signal Conditioning Board ................76

3.12 Detailed Schematic: Signal Processing Board .....................77

3.13 Photograph of PIC18F452 based Filter ................................78 
4.1 FIR FILTER: Data Acquisition Setup ………………………....79

4.2 FIR FILTER: Real-Time Testing Results ..................................80

4.3 LMS FILTER: Test Scenario 1 ......................................................86

CHAPTER 5: AnalySis \& CONCLUSIONS ...................... 92

5.1 FIR filter performance summery .............................................92

5.2 LMS filter performance summary ……………………………...94

5.3 Conclusions and future work .........................................................95

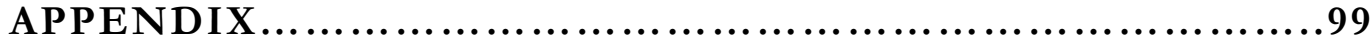

A USERS MANUAL FOR FIR FILTER DESIGN .................99

B MATLAB CODE FOR FIR FILTER DESIGN SOFTWARE..109

C CODE FOR $4^{\text {th }}$ ORDER FLOATING POINT LMS............130

D CODE FOR SWITCHED CAP CLOCK SOURCE..............143

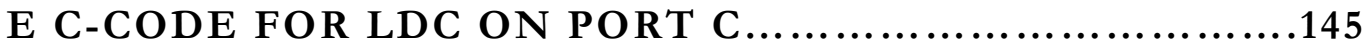

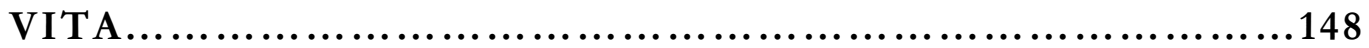




\section{LIST OF FIGURES}

Figure 1.5.1 Topology for Real-Time LMS Circuit Testing................ 4

Figure 2.1.1 Classification of Signal filtering methods ..................... 7

Figure 2.3.1 The transversal topology of the FIR filter.................... 8

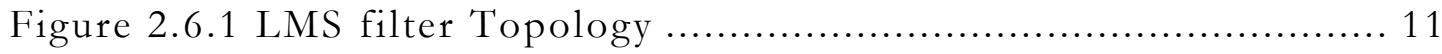

Figure 2.7.1 Standard Implementation of LMS Filter ..................... 12

Figure 2.8.1 Standard Implementation of Digital Filter.................... 13

Figure 2.8.2 Digital Signal Processing overview.......................... 13

Figure 3.1.1 FIR filter block Diagram .................................... 15

Figure 3.3 .2 FIR filter creation stages ................................. 17

Figure 3.3.2 Digital Signal Processing overview ......................... 18

Figure3.3.3 Frequency/phase review curves \& code generation ........... 19

Figure 3.3.4 Section of C-Code generated ................................. 19

Figure 3.3 .5 Intended LPF parameters ................................ 20

Figure 3.3.6 Skeleton code needed for Filter ........................... 20

Figure 3.3.7 Frequency and phase response plot ....................... 21

Figure 3.3 .8 Frequency and phase response plot......................... 21

Figure 3.3.9 Eight-Bit scaled coefficients .............................. 22

Figure 3.3.10 Circular buffer: Used to store ADC values for FIR filter. 25

Figure 3.3.11 Algorithm developed to load circular buffer................ 26

Figure 3.3.12 Step 1: Data element 0 is loaded and pointer decrements 26

Figure 3.3.13 Step 2: Data element 1 is loaded and pointer decrements 27

Figure 3.3.14 Step 3: Data element 3 is loaded and EOB is reached..... 27

Figure 3.3.15 Step 4: Element 4 is loaded and ptr is pre-decremented .. 27

Figure 3.3.16 Element 4 is unloaded from buffer and BOB is reached.. 28

Figure 3.3.17 Pointer is relocated to EOB and 3 is pulled ................ 28

Figure 3.3.18 Data element 1 is pulled................................... 29

Figure 3.3.19 Algorithm used to pull data from the circular buffer ...... 29 
Figure 3.3.20 Topology of the Double Circular Buffers .................... 30

Figure 3.3.21 Data Element 1 is loaded to both buffers................... 30

Figure 3.3.22 Data Elements $1-5$ are loaded to buffer..................... 31

Figure 3.3.23 Buffer Data Ready to be unloaded.......................... 32

Figure 3.3.24 Pointer FSR1 is relocated to same location as FSR0 ....... 32

Figure 3.3.25 FSR1 pre-increments and unloads data element $5 \ldots \ldots \ldots . . .33$

Figure 3.3.26 Algorithm for loading the adjacent circular buffers ........ 34

Figure 3.3.27 Assembly routine written to load the buffers................ 34

Figure 3.3.28 Algorithm used to unload from adjacent circular buffers. 35

Figure 3.3.29 Description of the ADCON0 Register..................... 37

Figure 3.3.30 Description of the ADCON1 Register..................... 37

Figure 3.3.31 Reading Only ADRESH will scale down to 8-bit ........... 38

Figure 3.3.32 RAM used by the FIR filtering scheme .................... 42

Figure 3.3.33 Description of the T1CON Register...................... 43

Figure 3.3.34 Word space dedicated to storing MAC result............... 45

Figure 3.3 .36 Multiply by 256 algorithm ............................... 46

Figure 3.3.37 Multiply by 128: Single Right shift of each byte ............ 46

Figure 3.3.38 Buffer setup for storing ADC samples ..................... 47

Figure 3.3.39 Buffer setup for storing Coefficients..................... 47

Figure 3.3.40 24-Bit Result store for MAC operation..................... 47

Figure 3.3.41 Multiply-Accumulate Algorithm .......................... 48

Figure 3.3.42 Initialization Routine for Fastest Execution ................. 49

Figure 4.3.43 Fastest Execution Implementation for PIC 18f452 ........ 50

Figure 4.3.44 RAM efficient Implementation for PIC 18f452............. 51

Figure 4.3.45 RAM Efficient Implementation for PIC 18f452 _........... 52

Figure 4.3.46 Minimum Program Size Implementation .................... 53

Figure 3.6.1 Assigned Word Length for Floating Point Format............ 55

Figure 3.6.2 Memory footprint of floating point numbers................. 57

Figure 3.6.3 Developed Algorithm for Multiplication .................... 58

Figure 3.6.4 Multiplication Step 1: $\mathbf{C}^{\text {sign }}=\mathbf{A}^{\operatorname{sign}}$ XOR $\mathbf{B}^{\text {sign }} \ldots \ldots \ldots \ldots \ldots \ldots$

Figure 3.6.5 Multiplication Step 2: $\mathbf{C}<$ FracL: FracH $>=A^{\text {fracH}} * B^{\text {fracH }} .59$ 


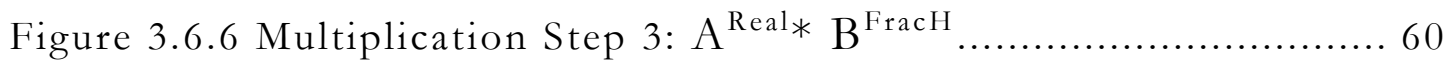

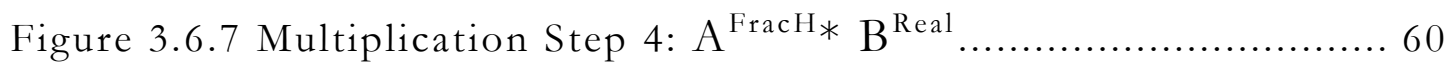

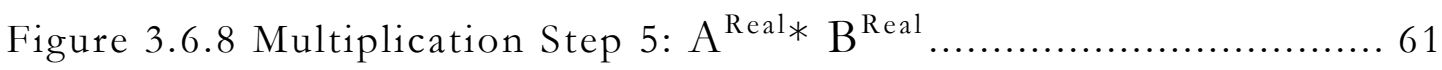

Figure 3.6.9 C-Code for floating point multiplication ..................... 61

Figure 3.6.10 C-Code for floating point addition.......................... 62

Figure 3.6.11 Developed Algorithm for Addition......................... 63

Figure 3.6.12 Developed Algorithm for Multiplication..................... 64

Figure 3.7.1 Fourth Order LMS Filter ...................................... 65

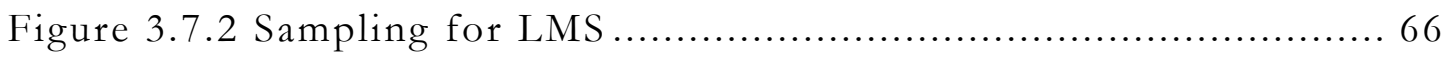

Figure 3.7.3 Configuring ADC for Sampling Two Channels ................ 67

Figure 3.7.4 Initialization Routine for LMS .............................. 68

Figure 3.7.5 Four element ADC sample bvffer for LMS ..................... 69

Figure 3.7.6 Arangements of Structs in Memory ............................ 70

Figure 3.7.8 Level 2 Flow Diagram for LMS ............................ 71

Figure 3.7.9 Level 3 Flow Diagram for LMS .............................. 72

Figure 3.8.1 Block Overview of Circuit .................................. 73

Figure 3.8.2 Power Supply Board ...................................... 74

Figure 3.8.3 Smoothing Filter for R-2R Ladder........................ 75

Figure 3.8.4 Signal Conditioning Block................................ 76

Figure 3.8.4 Block Overview of Circuit ................................. 77

Figure 3.8.5 Photograph of Test Board................................ 78

Figure 4.4.1 Basic setup for low-cost data acquisition .................... 79

Figure 4.2.1 Response Curves of Intended Filter ......................... 80

Figure 4.2.2 Test Signal: Constant Power Sweep [200Hz-4000Hz]....... 81

Figure 4.2.3 Filter Performance on PIC18F452 Chip ..................... 81

Figure 4.2.5 Measured BSF Frequency and Amplitude Response .......... 83

Figure 4.2.6 MBF 1 Filter Specifications for Band Pass filter ............. 84

Figure 4.2.7 Frequency and Amplitude response from PIC18F452 _..... 84

Figure 4.2.8 MBF 2 Filter Specifications for Band Pass filter ............. 85

Figure 4.2.9 Frequency and Amplitude response from PIC18F452 _...... 85 
Figure 4.3.1 Sampling Source A at P1 and P2 ........................... 86

Figure 4.3.2 Source $B$ is added to the scene.............................. 86

Figure 4.3.3 Approximation of Signal at p2 ........................... 87

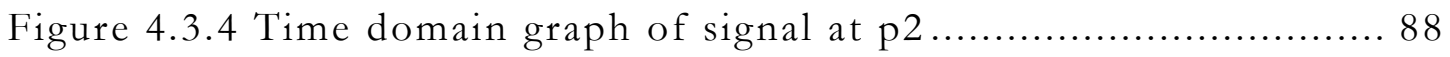

Figure 4.3 .5 Frequency domain graph of signal at p2 .................... 88

Figure 4.3.6 Real-time test topology ..................................... 89

Figure 4.3.6 Signal p2 (top) and Reference p1 sampled by ADC ......... 89

Figure 4.3.7 Signal Recovered by the PIC chop (source B: $340 \mathrm{~Hz}$ ) ..... 90

Figure 4.3.8 Signal Recovered in frequency domain (source B: $340 \mathrm{~Hz}$ ) 91

Figure 5.1 Comparison of exec speed for different implementations.... 94 


\section{LIST OF TABLES}

Table 3.2.1 Multiplication Speeds published by Manufacturer........ 16

Table 3.6.1 Floating point algorithms developed for LMS ........... 56

Table 5.1 LMS Sampling Rate vs Filter Taps.......................95 


\section{LIST OF NOMENCLATURE}

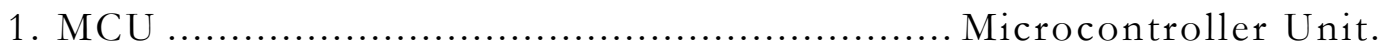

2. DSP ............................................ Digital Signal Processor.

3. ADC .......................................... Analog to Digital Converter.

4. DAC ....................................... Digital to Analog Converter.

5. PWM ............................................ Pulse Width Modulation.

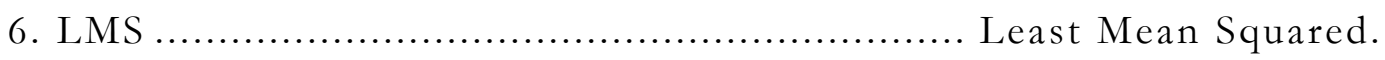

7. FIR ........................................ Finite Impulse Response.

8. IIR ....................................... Infinite Impulse Response.

9. ALU .......................................... Arithmetic Logic Unit.

10. MIPS ............................. Millions of Instructions per Second.

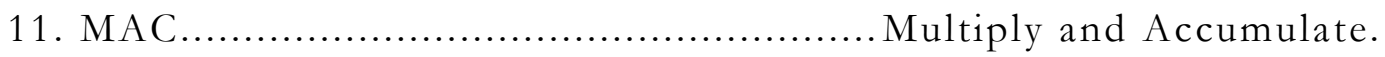

12. SMD ......................................... Surface Mount Technology.

13. RAM...............Random Access Memory/Read Write Memory.

14. EEPROM.Electrically Erasable Programmable Read Only Memory.

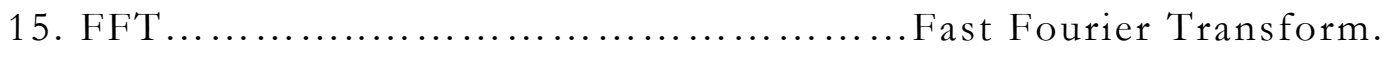

16. GUI................................. Graphical User Interface.

17. ISR .................................. Interrupt Service Routine.

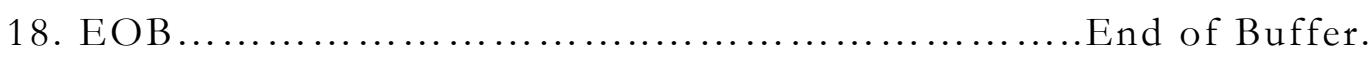

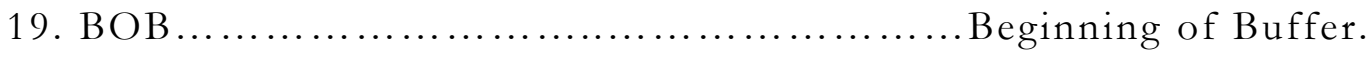

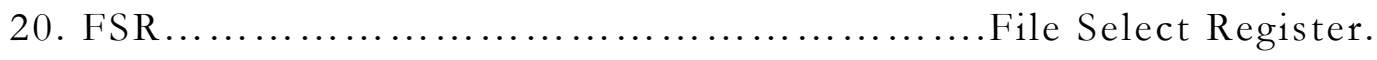




\section{CHA P TER 1: I N TRODUCTION}

\subsection{Personal Motivation}

My fascination with single chip microcontrollers began with my undergraduate days and has remained consistent ever since. In a world where minimalism is the catchword, they fit the role perfectly, being a cost effective way to elegantly solve complicated problems, thereby making so many aspects of electronics and software accessible to engineers and students as well. During my undergraduate and graduate years I participated in projects involving electronics and circuit design and always enjoyed finding new opportunities for microcontroller based solutions. With circuits getting more and more complex, filters must be installed to control noise and dealing with filters meant having to look for the right capacitors and the right resistors, op amps and repeating the same tedium all over again, especially if it was determined that a new set of filtering specifications were required. The alternative to a true digitalfilter is to use a switched capacitor filter but those are usually not as clean as analog filters and require a clock signal that adds switching noise; with an additional circuit component drawing power, occupying space and incurring an explicit monetary cost.

It is here that the Finite Impulse Response (FIR) filters step in, being an attractive alternative to using analog filters and switched capacitor digital filters. I learned about them in theory and conducted a couple of Matlab simulations before realizing that these are ideal for use with microcontroller projects. They impose no additional monetary cost upon the circuit; can be easily reconfigured by changing code, without any lag in performance with time. The challenge is to do a very efficient implementation for the PIC 18 architecture so it becomes possible for the filter to function as a supplementary application, thereby, providing 
an intuitive graphical interface that will allow anyone to easily generate these filters using a simple point and click system. However, not all noise problems can be solved by using FIR filters.

Sometimes due to the nature of the noise, especially if it is correlated, it is impossible for an ordinary (fixed-band) filter to remove it, because both the signal and noise occupy the same frequency range. For instance, if the echo of the signal was the source of noise, then the echo could not be removed simply by suppressing its frequencies, because the echo and the source have a strong correlation. In cases like these, adaptive filters are used to reduce noise. The least mean squared (LMS) error is a commonly used adaptive noise cancellation algorithm that is ideal for this purpose because it is a good compromise between computational complexity and performance.

\subsection{Signal Processing using the PIC 18F452 Microcontroller}

Microcontrollers such as PIC chips which run at speeds up to 10 MIPS (million instructions per second) are useful for gaining valuable practical experience with low bandwidth signal processing ideas. What makes them so convenient is the wealth of built in hardware, which can sample signals, perform ADC conversions and contain multiple timers for accurate timing. Moreover, there are a number of low cost compilers making the package available under $\$ 6.00$ per chip [4] and as low as $\$ 175$ for a C-Compiler and an in-circuit programmer for $\$ 75$ [2], making it feasible cost-wise as well. 


\subsection{Digital Filters Vs Analog Filters}

Digital filters have several advantages and disadvantages over their analog counterparts. The main advantage of digital filters is that they occupy no physical space as they are implemented completely in software and operate by applying a mathematical algorithm designed to produce the filtering effect. Since digital filters need no physical components i.e., capacitors and resistors, their performance does not degrade with age or respond to ambient environmental conditions. Another major advantage is that some digital filter (FIR filters) can have a unique property called linear phase response, which is critical in many communications applications. Analog filters presently, have a much greater dynamic range however, than digital filters because they are not limited by factors like sampling rate and computation speed [5].

\subsection{PIC 18 Microcontroller Family}

Microchip Technologies manufactures a popular line of micro controllers known as Peripheral Interface Controller or PIC chips. The PIC 18F452, released in May 2002, is currently one of their fastest chips [3]. At the core of this chip is an 8-bit RISC based ALU that can process 10 MIPS at $40 \mathrm{MHz}$. Its design is based on Harvard architecture, allowing it to have separate data and program memories. Its memory is divided into $32 \mathrm{~KB}$ of flash based program memory and $1.5 \mathrm{~KB}$ of volatile data memory (RAM) as well as 256 Bytes of EEPROM. PIC chips have a RISC based instruction set consisting of a small yet seminal set of instructions, most of which are single cycle, thereby making them fast executing and easy to program. Other valuable devices such as analog to digital converters, pulse width modulation, multiple timers, I/O Ports are all integrated within the same chip that also contain hardware support for several popular serial communication protocols 
such as $\mathrm{I}^{2} \mathrm{C}$, SPI and UART. Running at $40 \mathrm{MHz}$, it takes the $18 \mathrm{~F} 452$ chip 100 ns to multiply two bytes and compute a 16-bit result. The other noted feature that makes this chip viable for signal processing applications is that, it contains multiple hardware pointers that allow very fast access to data stored within the chips' RAM.

\subsection{Detailed Research Objectives and Contributions}

The main focus of this research will be to test and validate the PIC chips' ability to implement a real-time floating-point LMS based Adaptive filter, which is a very useful way to deal with noise that is too closely related to the signal for conventional band compensating filters to handle.

1. A suitable general-purpose, adaptive noise cancellation circuit will be designed, that is both cost effective and customizable to serve several different applications. The circuit will be tested using test signals generated by a PC sound card as shown in Figure 1.5.1. This will allow the modeling of different types of noise and to test various signal to noise ratios. The circuit will process the signals in real-time and the results will be measured using a data acquisition system. Analysis of the recorded data should reveal the effective noise reduction versus noise reductions predicted via simulations.

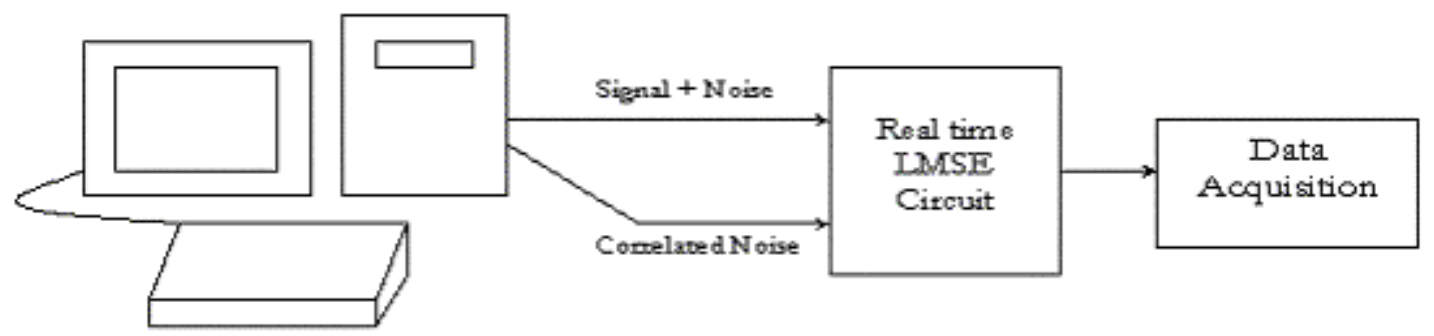

Figure 1.5.1 Topology for Real-Time LMS Circuit Testing 
2. During the course of this research, software will be developed to benefit the users of the popular CCS PIC Compiler. The software will include a modular library for PIC 18XXX with optimized floating-point math support. Although the compiler is inherently capable of handling floating-point data, it performs common floating-point operations such as addition, subtraction, and multiplication at an alarmingly slow rate rendering it unpractical for real-time applications. Therefore, another aim of this research will be to develop a modular library that will provide a faster alternative to the compilers built in floating-point system.

3. The secondary focus of this research will be to test and validate the PIC chips' ability to implement a real-time fixed-point FIR filter, which is a very practical idea, because it can be seamlessly used in countless applications where noise and the signal of interest occupy separate frequency bands.

4. A Graphical User Interface (GUI) will be developed that allow users to design various types of FIR filters, such as Low-Pass, High-Pass, Band Pass, Notch or any combination of the above, in short, multi-band filters. The user may design the filter by taking a point and click approach to specifying band-edges, attenuations, sampling rate etc. and the software will show users the respective frequency and phase response graphs. Once the user is satisfied with the filter they have designed, the software will present them with several realization options, thereby allowing them to decide whether they want the filter optimized for execution speed, or conservative RAM usage or conservative programmemory usage. Ultimately, optimized C language source code is generated that is ready to be compiled for either PIC $18 \mathrm{~F} 452$ chip or the smaller PIC 18F252 chip or easily adapted for the remaining chips in the PIC 18FXXX family by a moderately experienced programmer. Finally, the GUI will generate a diagram of the test circuit needed to install the filter code. 
5. Each type of FIR filter created by the software will be evaluated independently by applying a constant-power frequency sweep generated by a filter test program. The real-time output of the filter will be recorded by a data acquisition system and its performance will be analyzed though PC based data analysis tools such as FFT.

\subsection{Organization}

Chapter two will cover a literature review and theoretical background of existing techniques for digital filter implementation for both LMS and FIR filters. Chapter three will constitute the implementation details for both the filters. Chapter four will present results and analysis and chapter five will contain recommendation for future work. An appendix is provided that contains all codes written during this exploration and a user's manual for the filter design of the GUI. 


\section{CHA P T ER 2: L I T ERATURE S URVEY}

\subsection{Classification of Filtering Methods}

The earliest filters were analog filters. In recent years, digital filters have gained popularity due to the lowering cost of microprocessors and the increased level of convenience and flexibility offered by digital filters. Advances in technology allowed them to function at a faster speed and now they are rapidly approaching the large dynamic range of analog filters [5]. A broad classification of Digital filters is presented in Figure 2.1.1.

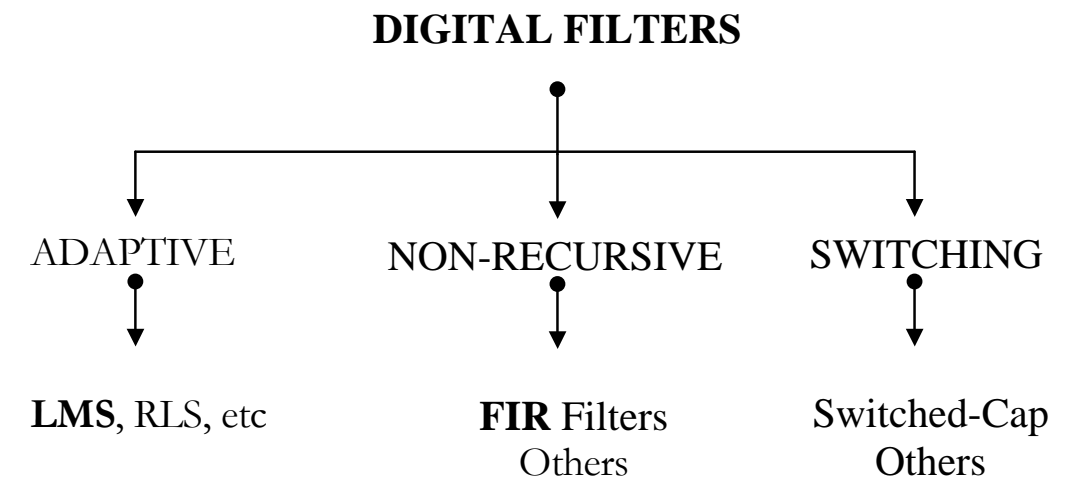

Figure 2.1.1 Classification of Signal filtering methods

\subsection{Digital Filters}

A digital filter is a discrete-time linear system that operates on an input sequence, modifies it, and produces the output sequence. The input sequence is usually obtained by digitizing a signal, thereby converting it into discrete time, with the output sequence being transformed back into an analog signal through an appropriate digital to analog process. The steadily reducing cost of portable computation is 
thereby making a direct contribution to the rise of popularity of digital filters.

\subsection{Non-Recursive Type Digital Filters}

The most commonly used Non-Recursive filter is the FIR filter. The weights of this type of digital filters are constant and are computed at design time. Since the weights remain constant, the stability of FIR filters can be guaranteed. However, they can have several topologies the transversal topology as shown in Figure 2.3.1 being the most common type and the one used for this research [9].

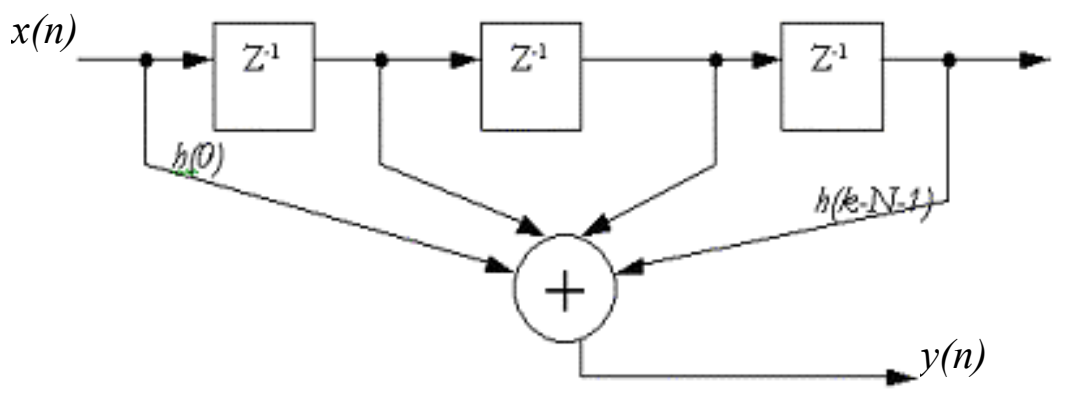

Figure 2.3.1 The transversal topology of the FIR filter

The transversal FIR filter is characterized by the following equation.

$$
y(n)=\sum_{k=0}^{N-1} h(k) * x(n-k)
$$

Where,

$x(n)$ : discrete time elements of the sampled signal

$y(n):$ is the computed output of the FIR filter

$b(k)$ : are the coefficients of the filter also knows as filter-taps 
Linear convolution of the filter coefficient with the sampled signal produces the filtering effect. Since multiplication and addition are the only mathematical operations involved with the FIR filter, this process is ideally suited for use within the PIC 18F452 microcontroller. The clear advantage of using FIR filters is the radical alteration in its frequency compensation, which can be achieved by simply providing the system with a new set of filter coefficients. Another interesting property of FIR filters is that, they are the only type of filter that can have a true linear phase response. Since this research deals exclusively with the implementation aspects of FIR filters, it is assumed that the coefficients of the filter have already been computed. For more theoretical details regarding obtaining filter coefficients refer to [7].

\subsection{Switching type digital filters}

The switched capacitor filter is a common type of switching filter. Switching type digital filters are a convenient alternative to using high order analog filters. They are packaged for convenient use and typically require a clock signal and power to operate. Most are strictly low-pass filters; others can be programmed by additional resistors, to be used as band pass and notch filters. However, this convenience comes at the expense of additional monetary cost and components and having to deal with the inescapable incurrence of switching noise [1].

\subsection{Adaptive Filters}

One of the most successful adaptive algorithms is the LMS filter developed by Widrow [14]. LMS, sometimes known as LMSE is excellent for dealing with correlated noise where noise and the signal are too much alike to be filtered using ordinary band-compensating filters such as lowpass, band-pass etc. Such filters are commonly referred to as adaptive 
filters and they are used in applications such as, echo-cancellation over communication lines, noise-cancellation, Electro-cardiogram (ECG) in pregnant mothers, suppressing machine noises in mines and countless other applications.

\subsection{Least Mean Squared Error (LMS)}

The LMS filter is based on the steepest decent algorithm where the weight vector is updated from sample to sample as follows:

$$
W_{k+1}=W_{k}-\mu \nabla_{k}
$$

where,

Wk: Is the weight vector

$\nabla_{k}: I s$ the true gradient vector

$\mu$ : Rate of convergence also referred to as learning rate

The LMS algorithm is a practical method of obtaining estimates of the filter weights $\mathrm{Wk}$ in real time. The Widrow-Hopf LMS algorithm for updating weights from sample to sample is given by:

$$
W_{k+1}=W_{k}+2 \mu e_{k} X_{k}
$$

where,

$$
e k=y_{k}-W_{K}^{T} X_{k}
$$

ek: Is the error term

$X_{k}$ : Is the correlated noise vector

LMS algorithm above does not require prior knowledge of the signal statistics, but instead uses instantaneous estimates to tune the filter. The weights obtained by the LMS algorithm only estimates, but these 
estimates improve gradually with time as the weights are adjusted and the filter adapts itself to the characteristics of the signals. Eventually, the weights converge. The condition for convergence is,

$$
0<\mu<\frac{1}{\lambda \max }
$$

where,

$\lambda$ max: Is the maximum Eigen value of covariance matrix.

The main objective in adaptive noise cancellation is to produce an optimum estimate of the correlated noise in the contaminated signal. This is done by the simultaneous sampling of two signals - one being the signal of interest to be filtered and the other being the source of correlated noise, referred to as the reference. The adaptive filter in Figure 2.6.1 uses the reference to predict the degree of contamination in the signal of interest by the process of correlation.

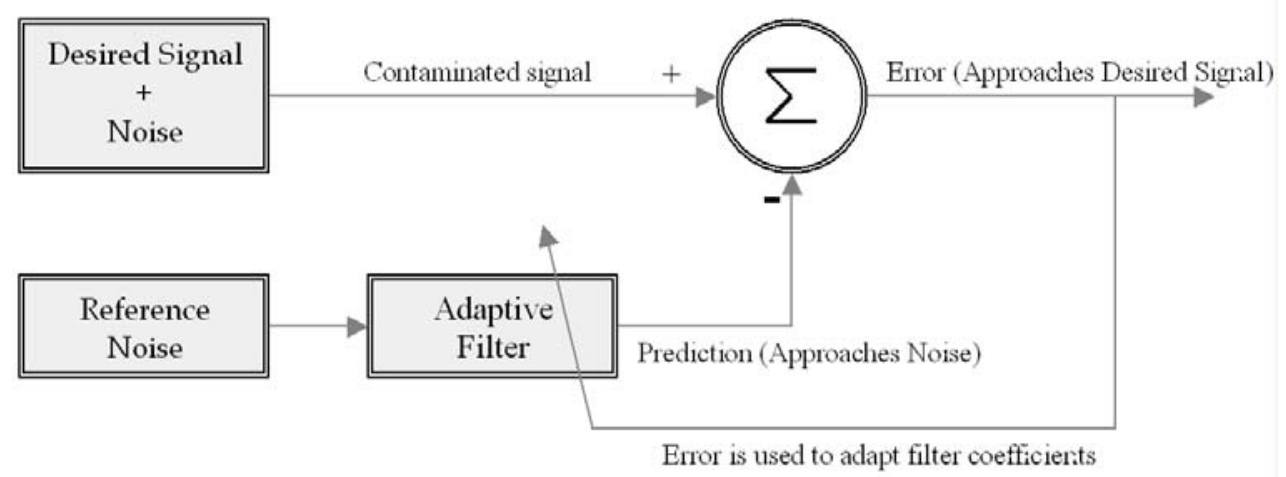

Figure 2.6.1 LMS filter Topology

The adaptive filter attempts to predict the amplitude and phase of the noise present in the contaminated signal by correlating the reference with the contaminated signal. The prediction of the adaptive filter constantly approaches the actual noise present in the contaminated 
signal. With the error signal continuously being used to tune the filter, it gradually approaches the desired signal. Figure 2.7.1 shows a flow diagram for the LMS filter algorithm.

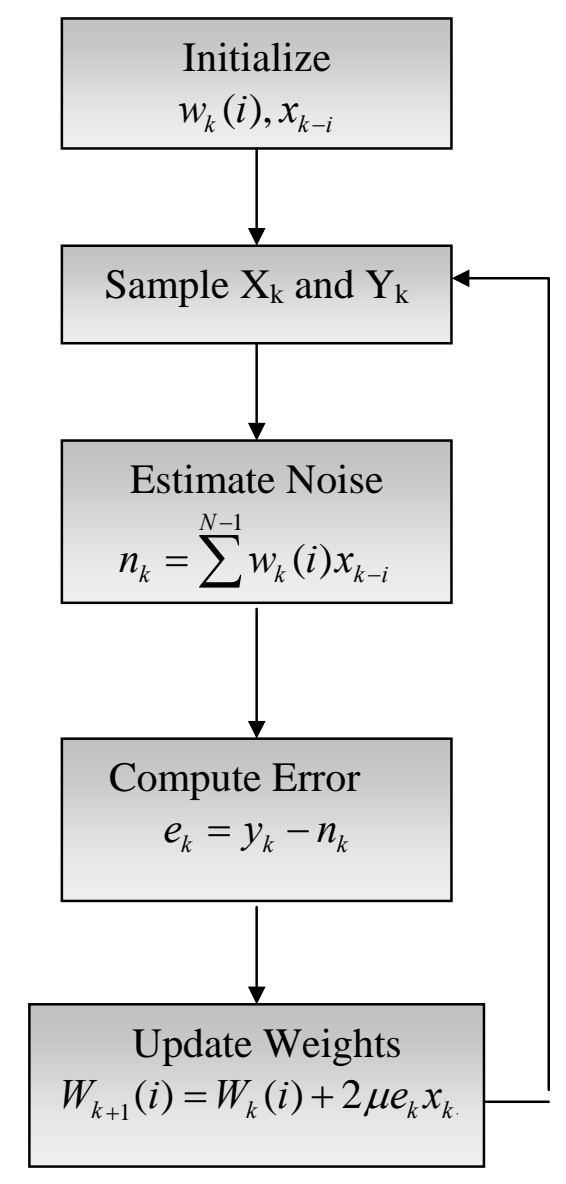

Figure 2.7.1 Standard Implementation of LMS Filter

\subsection{Implementation of a digital filter}

Digital filters are a natural choice for circuits that are interfaced to or controlled by a microcontroller. Part of the microcontroller's computing power may be dedicated to filtering the sampled input signals. Figure 2.8.1 is a block diagram for typical digital filter implementation. 


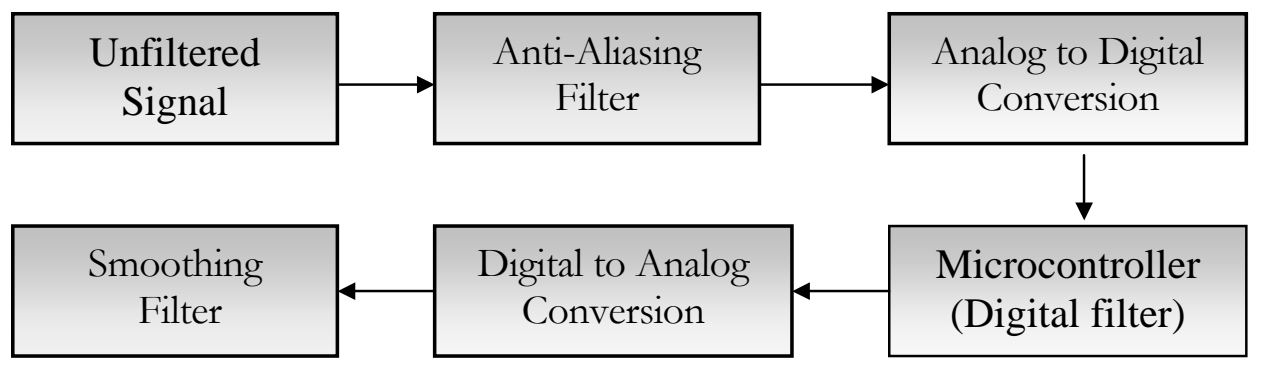

Figure 2.8.1 Standard Implementation of Digital Filter

The Anti-Aliasing filter is a low-pass filter designed with a cutoff that is at least half the sampling rate of the analog to digital converter (ADC). This is used to prevent sampling of frequencies above Nyquist rate [6]. A smoothing filter is another low-pass filter that is used to reduce the harmonic distortion resulting from the quantization process. An illustration of the described process is presented in Figure 2.8.2.

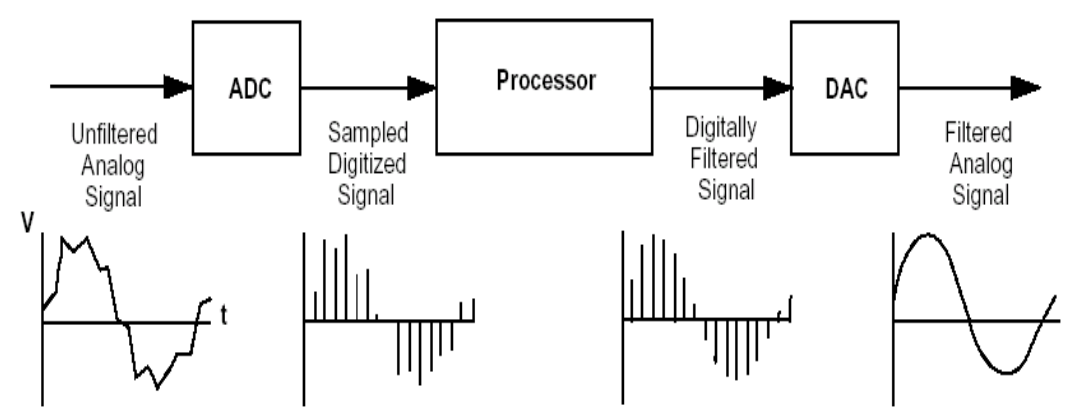

Figure 2.8.2 Digital Signal Processing overview 


\section{CHAPTER 3: IMPLEMENTATION DETAILS}

Implementation details for the fixed-point FIR filter are discussed first followed by the implementation details for the more complicated floating-point LMS filter.

\subsection{Finite Impulse Response (FIR)}

Development of a FIR filter generally involves two distinct phases. The first one is the design phase and the other is the realization phase. The design phase involves specifying filter characteristics such as bandedges, frequency-response and phase-response etc. and finally derives the filter coefficients for the intended filter. There are several ways to obtain filter coefficients. For this research the Matlab filter design toolbox was used to generate them. In FIR filters, the same hardware can be used to realize many different types of filters. It can be seamlessly reconfigured from a low-pass to band-pass to notch or a combination of all of them by simply supplying a new set of coefficients. The implementation discussed in this study is optimized for the PIC 18 F452 instruction set although it is flexible enough to be easily adapted to other inexpensive microcontrollers with similar hardware.

The software developed for building FIR filters includes a program that allows users to visually specify the filter parameters. Once the filter has been finalized, optimized code will be automatically generated for the PIC $18 \mathrm{~F} 452$ processor. Since the filter is usually used as a supplementary application, it must be designed to co-exist with a main application. The proposed implementation uses only a fraction of the microcontrollers' total computational capacity and the remaining cycles are reserved for the main application. Additionally, the implementation scheme is easy to reconfigure without making changes in hardware. 
The second phase is the realization phase. This involves the selection of an appropriate platform upon which the filter will be implemented. In this case the platform desired is the PIC 18 F452 chip. Real-time implementation involves three distinct processes - firstly the analog-todigital conversion of a signal; followed by mathematical processing by the filtering algorithm; and finally, if needed the obtained results have to be transformed back into an electrical signal using a suitable digital-toanalog conversion technique. All three processes mentioned above must be performed within a proper time constraint or the result becomes invalid. For instance, if we are sampling a signal at $4000 \mathrm{~Hz}$ then our worst-case time is $1 / 4000 \mathrm{~Hz}$ or 250 us. All filter computations must be completed within the time window of 250 us. The block diagram of the FIR filter is presented in Figure 3.1.1.

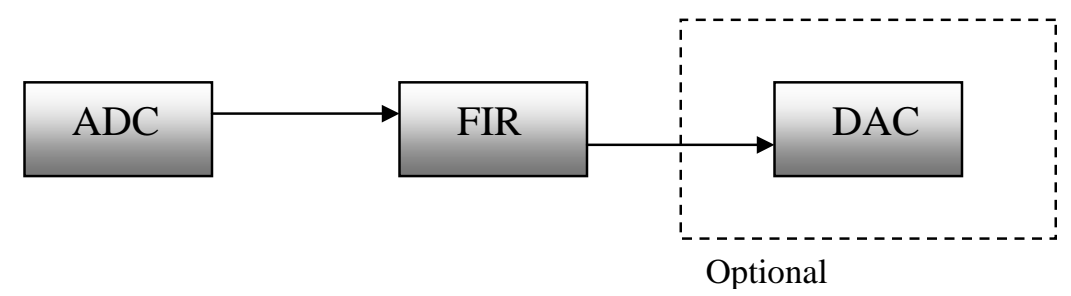

Figure 3.1.1 FIR filter block Diagram

\subsection{Implementation Background}

Three different implementation strategies are provided to the user as options, each with its advantages and drawbacks. They are minimum RAM implementation, minimum program memory implementation and minimum execution time implementation. Each will be discussed in detail in the following sections.

Implementation aims to take advantage of the PIC chip's hardware architecture and instruction sets. The PIC 18 F452 chip has certain features in its hardware that makes it a good choice for filtering 
applications. The following restrictions were used while implementing the FIR filter algorithm in order to maximize the filter throughout.

1. Multiplication operations are restricted to unsigned integer data only. The Table 3.2.1 is a summary of manufacturer published multiplicationperformance for the PIC 18F452 chip [10]. Table 3.2.1 outlines the speed gain from using the hardware multiplier and by favoring unsignedmultiplication operations instead of signed multiplication operations. Time $_{\mathrm{H}}$ is the time needed performing hardware multiplication and Time is the time needed to perform software multiplication.

Table 3.2.1 Multiplication speeds for PIC18452

\begin{tabular}{|c|c|c|c|}
\hline ROUTINE & METHOD & Time $_{\mathrm{H}} /$ Time $_{\mathrm{s}}$ & Speedup \\
\hline $8 \times 8$ Unsigned & Hardware/Software & $100 \mathrm{~ns} / 6.9 \mu \mathrm{s}$ & $6900 \%$ \\
\hline $8 \times 8$ Signed & Hardware/Software & $600 \mathrm{~ns} / 9.1 \mu \mathrm{s}$ & $1500 \%$ \\
\hline $16 \times 16$ Unsigned & Hardware/Software & $2.4 \mu \mathrm{s} / 24 \mu \mathrm{s}$ & $1000 \%$ \\
\hline $16 \times 16$ Signed & Hardware/Software & $3.6 \mu \mathrm{s} / 25.4 \mu \mathrm{s}$ & $1400 \%$ \\
\hline
\end{tabular}

2. The analog to digital converter is used with 8-bit resolution. Even though the built in ADC on the PIC chip is capable of sampling up to 10-bit resolution, the PIC memory and ALU are both 8-bit wide. It is therefore most efficient in handling 8-bit data. Hence, all filter coefficients and ADC data will be restricted to 8-bit resolution.

3. All memory references are made using indirect addressing. The PIC 18F452 chip contains three hardware pointers. FSR0, FSR1, FSR2, each being 12 Bits and capable of covering the entire RAM size for the PIC 18 family (up to 4096 bytes for PIC18f2515). By shortening the range of these pointers to 8 -bits we can gain efficiency at the expense of smaller memory coverage. The pointer space will be restricted to 8-bits to cover 
256 bytes of RAM or a single bank of RAM. This means that all our buffers and other dynamically allocated areas of RAM have to be confined to 256 bytes of memory.

\subsection{FIR Filter Implementation}

FIR filter implementation scheme on the PIC 18 F452 chip can be categorized using the following major steps shown in Figure 3.3.1.

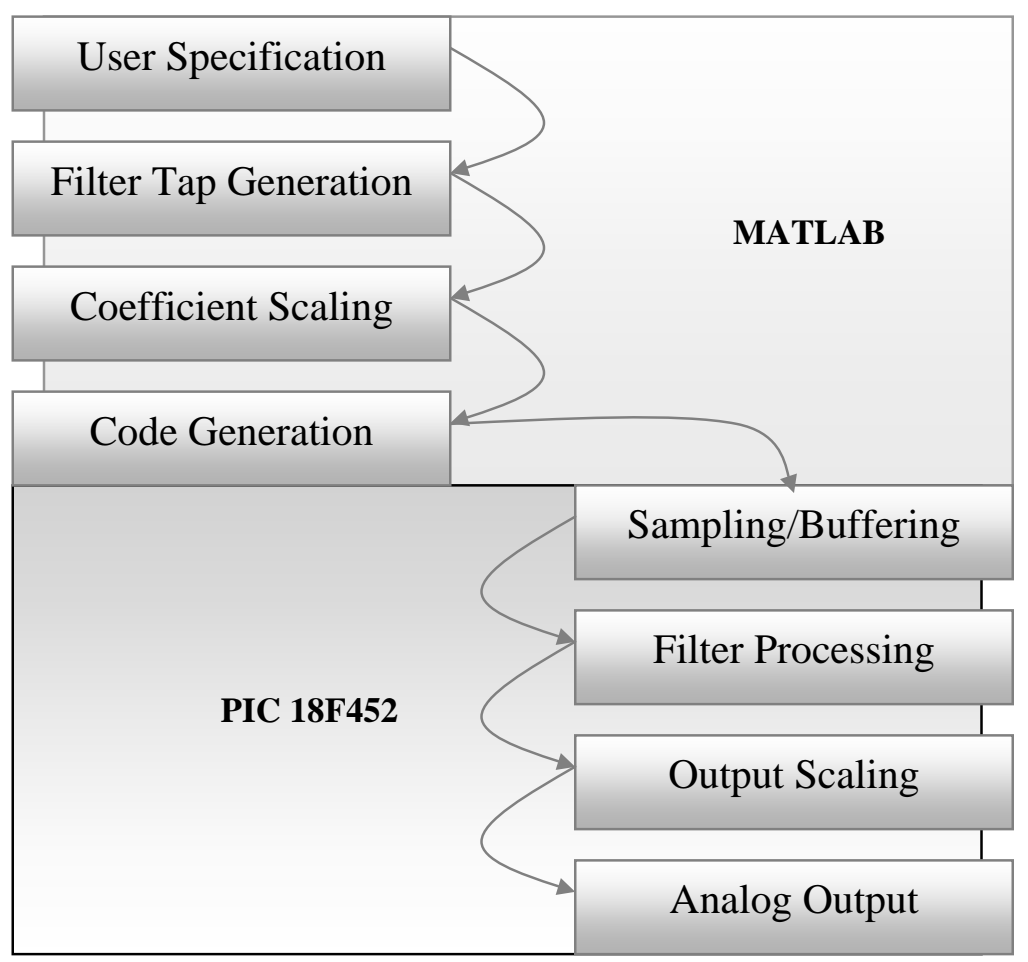

Figure 3.3.2 FIR filter creation stages

\subsubsection{User specification}

The very first logical step to making a filter is to specify filter parameters such as band edges, attenuations and ripples. To this end, the following interface was developed to allow a user to specify the type and 
exact parameters of the filter to be designed. Figure 3.3.2 is a snapshot of the developed filter making software.

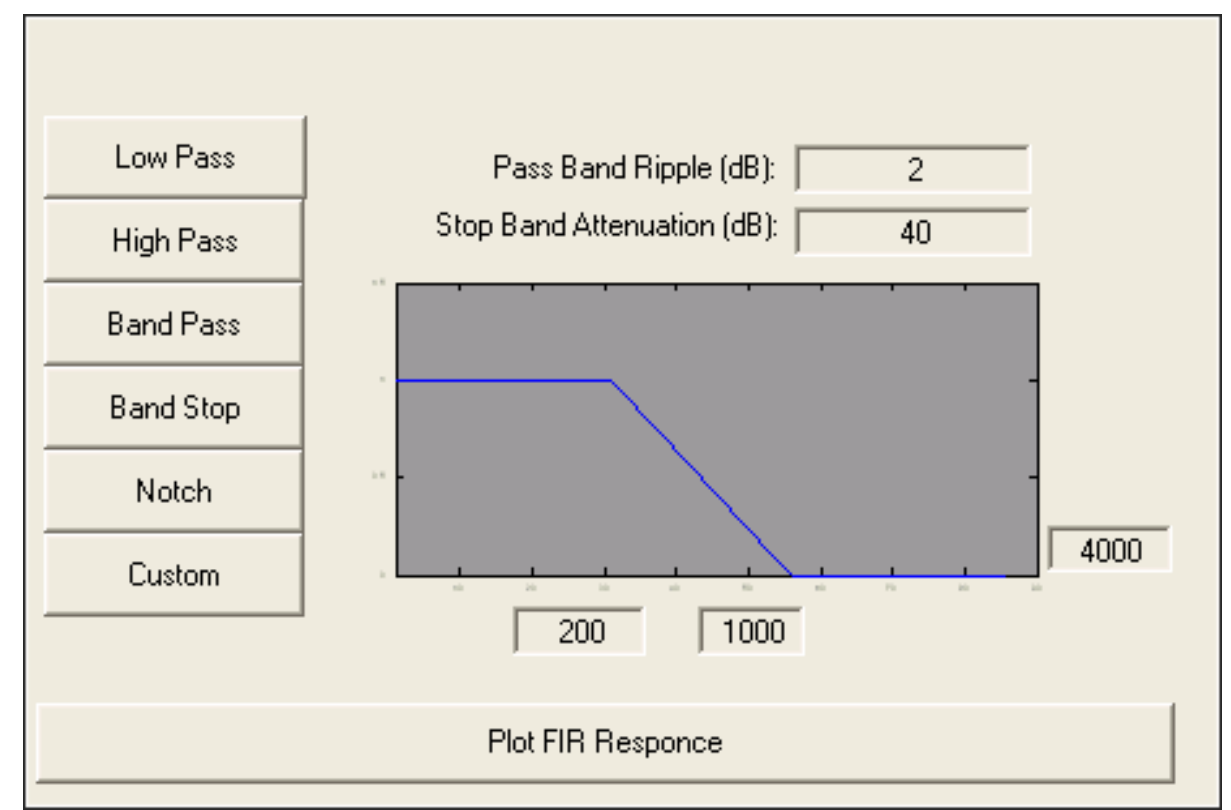

Figure 3.3.2 Digital Signal Processing overview

By making use of the menus the user can select from the range of filters that can be generated for real-time implementation. The available options are low-pass, high-pass, band-pass, band-stop, notch and custom. Once the type of filter is decided, the user can specify parameters such as band edges and attenuations by filling in the appropriate boxes. Before the user is allowed to generate code, the frequency and phase response for the desired filter circuit must be reviewed. The software automatically calculates the exact filter order required to achieve filtering requirements. The filter coefficients are calculated using the Remez exchange [8] method for optimal tap generation for low-pass and highpass configurations. Figure 3.3 .3 shows the frequency and phase response curves as well as the different code generation options available to the user. If satisfied the user may generate the desired filter. 


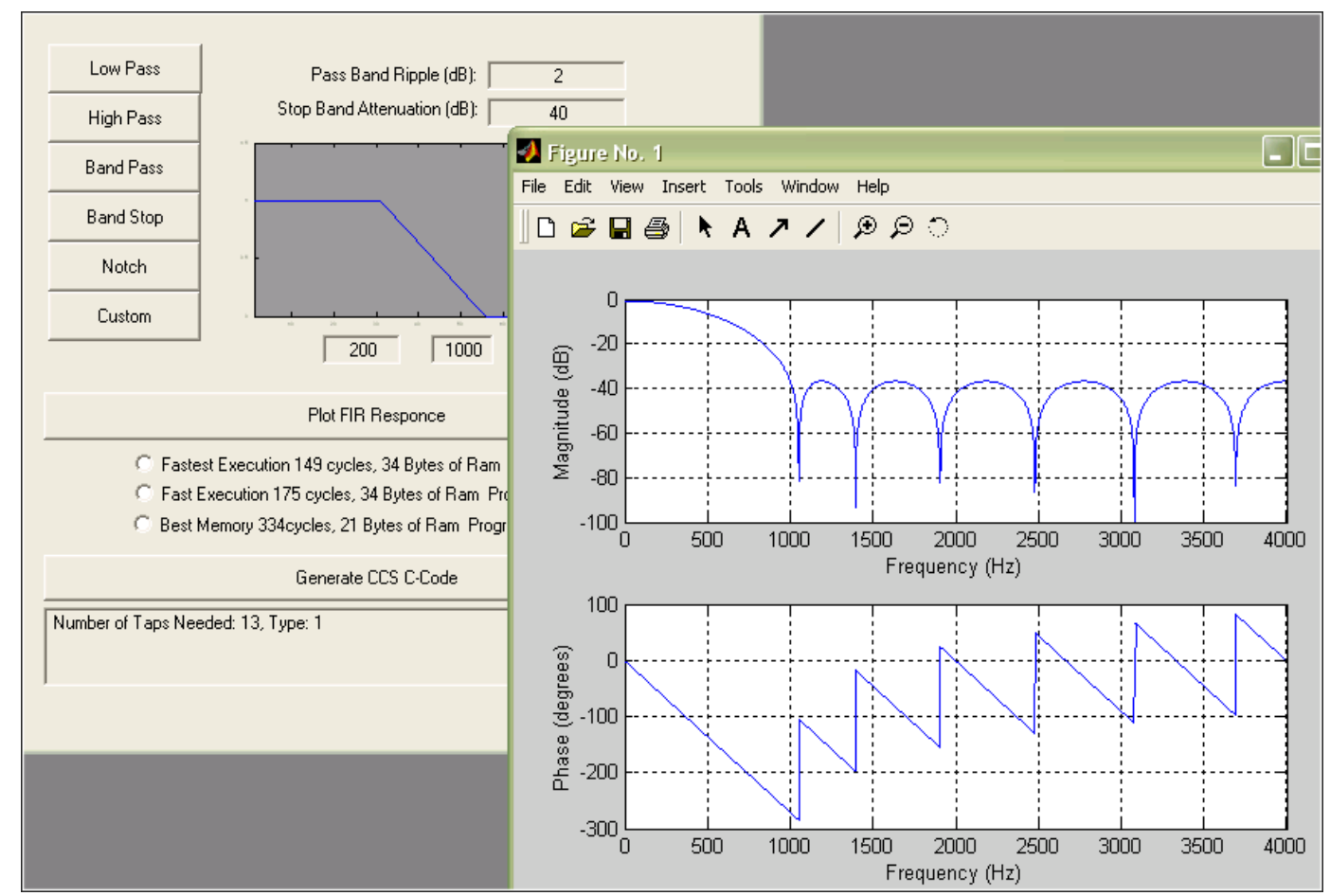

Figure3.3.3 Frequency/phase review curves \& code generation options

Once a satisfactory design is achieved the user is given three options for code generation. Finally C-language code, as shown in Figure 3.3.4, is generated that is ready to be compiled or edited.

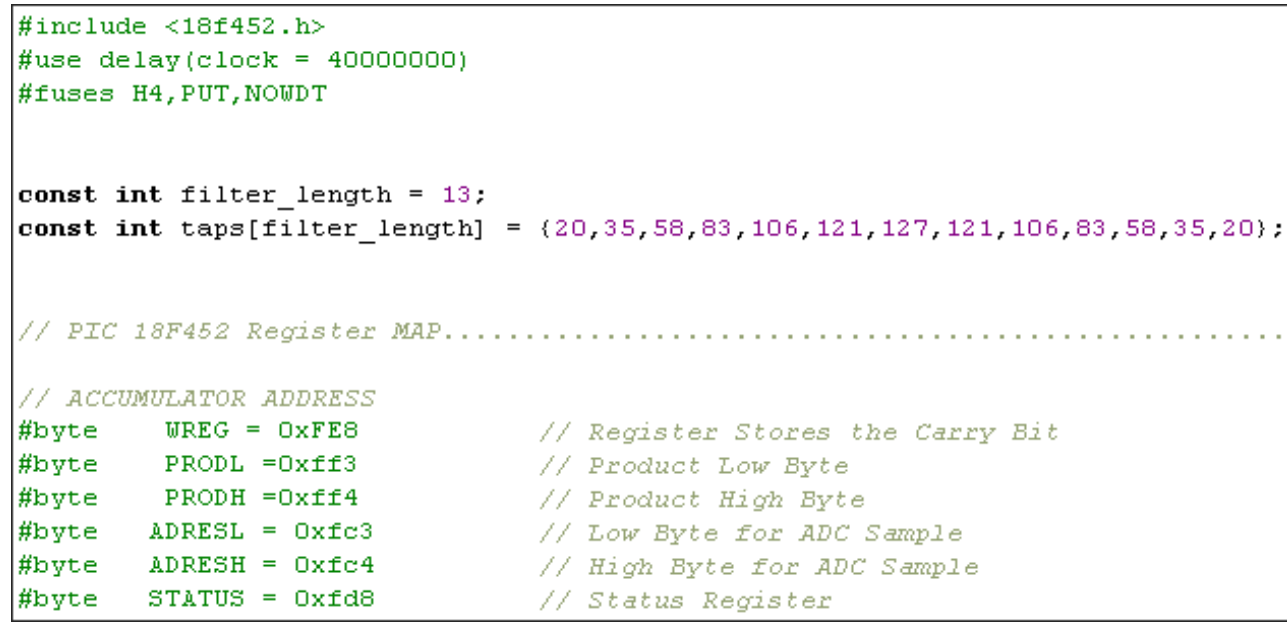

Figure 3.3.4 Section of C-Code generated 


\subsubsection{Filter Tap Generation}

The Matlab filter design toolbox [13] was used to generate filter coefficients. This toolbox contains a set of functions that allow users to conveniently make and test different types of filters. If for example, a low-pass filter was desired with the characteristics given in Figure 3.3.5 and Figure 3.3.6 is the skeleton Matlab-code needed to generate it.

Sampling Frequency of $8000 \mathrm{~Hz}$

Pass band frequency of $500 \mathrm{~Hz}$

Stop Band frequency of $1000 \mathrm{~Hz}$

Pass band ripple of .05 dB

Stop band ripple of $55 \mathrm{~dB}$

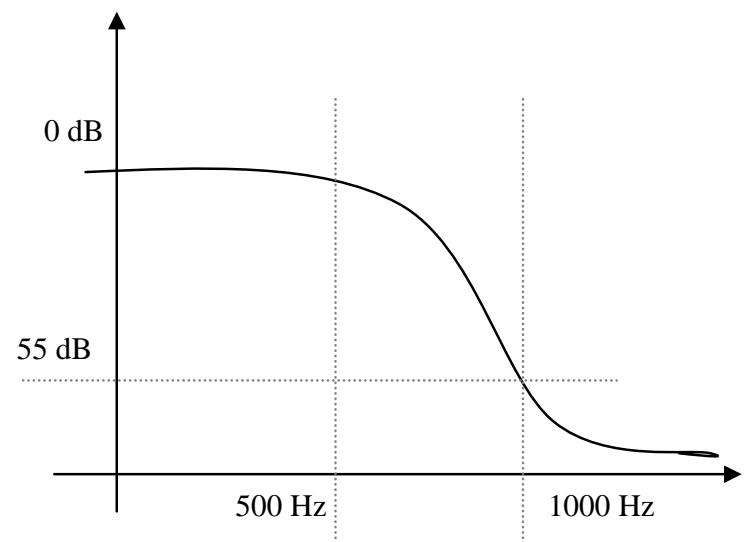

Figure 3.3.5 Intended LPF parameters

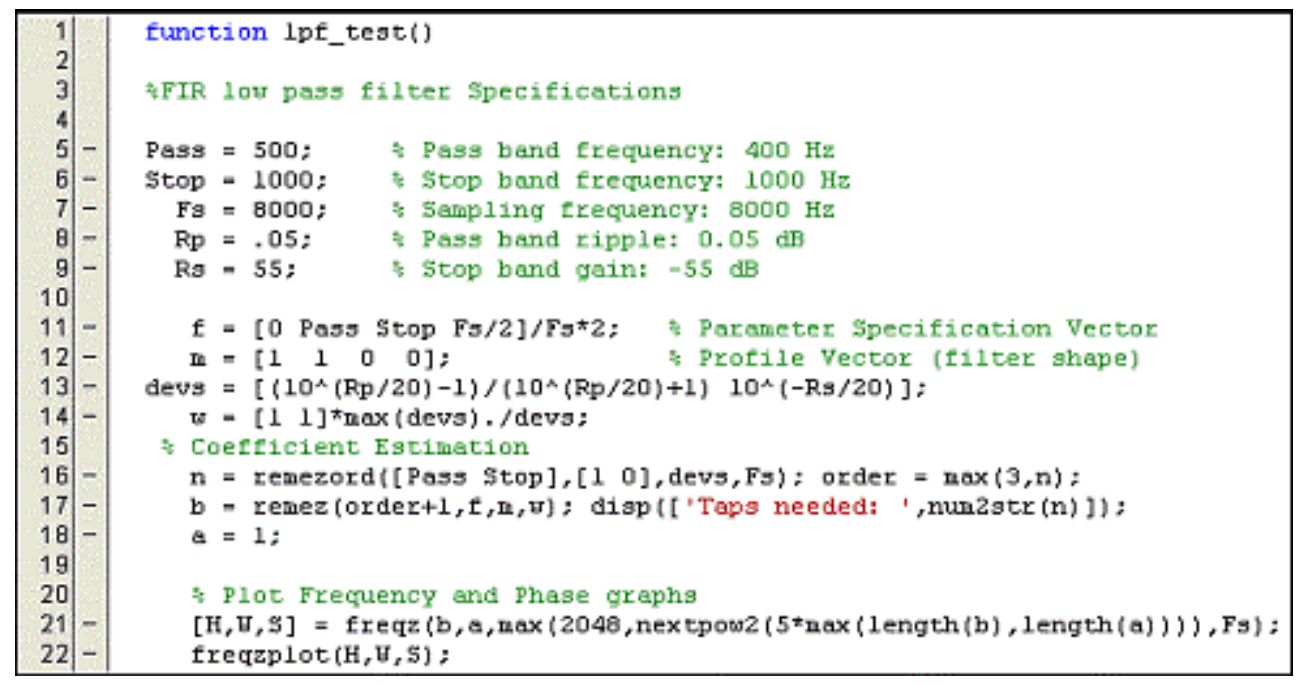

Figure 3.3.6 Skeleton code needed for Filter 
The skeleton code presented in Figure 3.3.6 upon execution will produce the graphs for both phase response and frequency response in Figure 3.3.7.

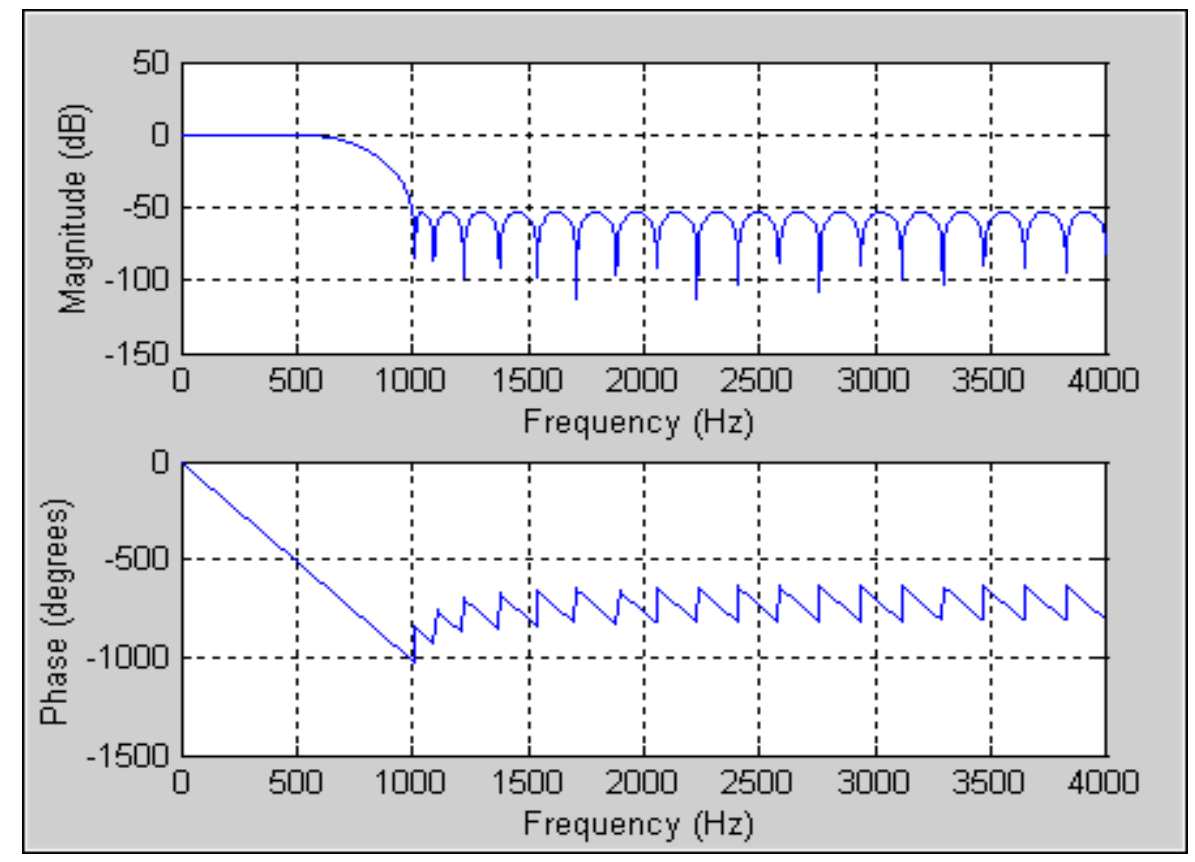

Figure 3.3.7 Frequency and phase response plot

The filter tap coefficients generated by Matlab are as plotted next:

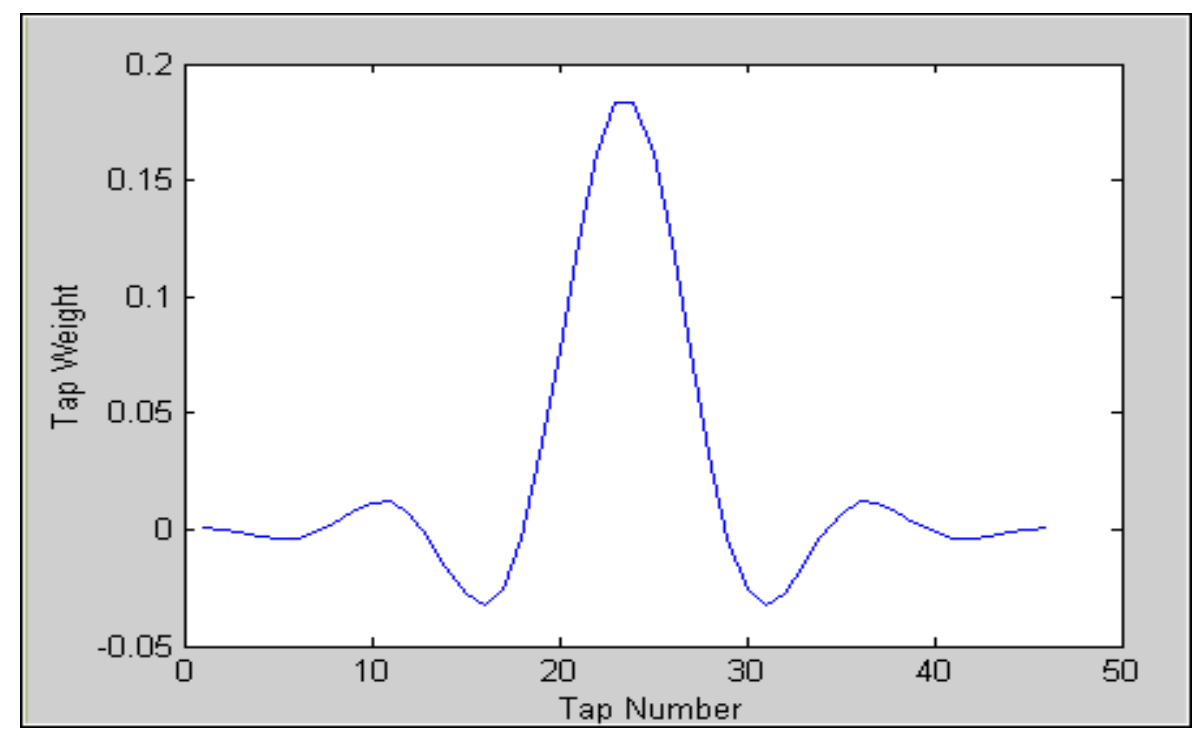

Figure 3.3.8 Frequency and phase response plot 


\subsubsection{Coefficient Scaling}

The tap coefficients computed by Matlab are computed in floating point format ranging from $[-1.0,1.0]$. Before they can be used in the PIC chip they need to be converted into 8-bit fixed-point format and made unsigned. The following scaling function apply the to achieve this:

$$
\text { scaled_tap }{ }_{n}=\text { ceiling }\left\{\frac{\text { floating_tap }}{\max (\text { floating_tap })} * 127\right\}+128
$$

Each tap coefficient provided by Matlab is first normalized to the range $[-1.00,1.00]$, then multiplied by 127 and rounded to the higher integer. Finally 128 is added to each tap to make it positive. After the scaling function is applied, the [-1.00,1.00] range becomes $[0,255]$, shown in Figure 3.3.9 and now unsigned integers.

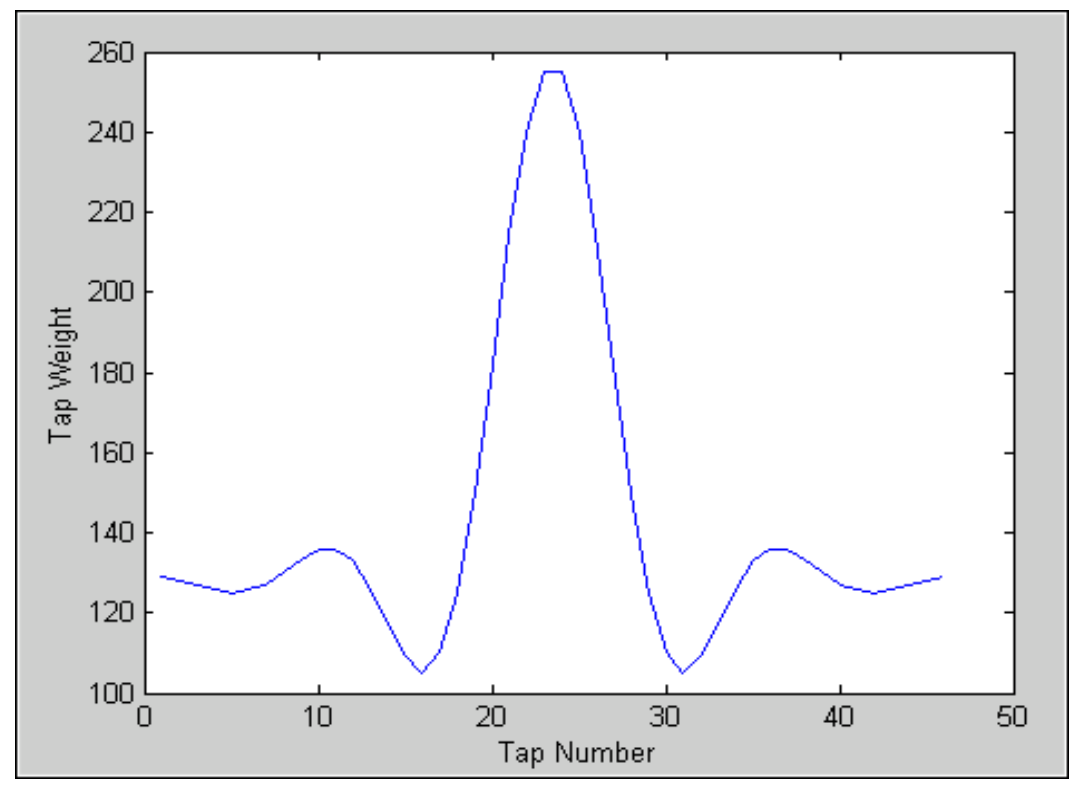

Figure 3.3.9 Eight-Bit scaled coefficients 


\subsubsection{Code generation}

Once the filter parameters are established and all decisions involving implementation details are complete, compiler ready C-language source code for the CCS PIC C is generated based on a set of three different templates. Each template is a specialized implementation scheme optimized to produce a different flavor.

1. Minimum Ram: The c-code generated using this template makes minimal demand on RAM.

2. Minimum Program Size: This template minimizes the program size.

3. Minimum Execution Time: This template produces code that achieves higher execution speed.

If the PIC chip is dedicated to performing signal filtering only then either implementation scheme is suitable. However if the FIR filter is used as a supplementary application that runs along side a primary application then it competes for the same recourses as the main application. Thus it may be useful for the user to use the parametric optimizations. To facilitate the selection of which type of optimization is suitable the user interface provides exact values for RAM, program memory and execution time with each option. The optimizations in RAM, execution speed and program size were all derived using a combination different buffering techniques, loop unrolling, and inline assembly language routines for the real-time components. Each is discussed in detail in the following sections. 


\subsubsection{Buffering Data}

Once Matlab has generated the filter coefficients, they need to be accommodated within the PIC memory. Additionally, the constant stream of data from the PIC ADC must be accommodated in memory with the exact chronological sequence in which it was sampled. The buffering scheme for tap coefficients is discussed first.

The tap coefficients are stored in the PIC in the form of a look-up table in its program memory. Before filtering begins, the entire table is copied to the RAM and marked with a hardware pointer. Managing coefficients is not complicated because the number of taps is finite and the list is static (needs to be initialized only once).

Buffering the ADC data is a far more interesting problem. There are several complications that have to be dealt with. The finite impulse response filter is quite simply the linear convolution between a constant set of filter taps and a discrete time capture of a signal. For example, say, the desired filter has 30 tap coefficients then we would need to capture and store not only a latest sample of the signal, but the previous 29 samples as well. To achieve this, two different buffering schemes were explored. The first one used a traditional one-dimensional circular buffer [11]. This technique uses less memory but lengthens the cycle of computations. The second technique used two adjacent one-dimensional circular buffers [12]. This technique uses more RAM than the first, but allows the speed of the filter to approach its shortest possible computation time on the PIC 18 F452 chip (using 8-bit taps and 8-bit data).

1. Circular buffer implementation on PIC 18F452: A circular buffer is a memory allocation scheme where memory is reused (reclaimed) when an index is incremented to a multiple of the buffer size. The modulo 
nature of a circular buffer maintains data in a queue form (chronological order) at all times without overrunning its allocated memory or the need for re-ordering. The elegance of this type of memory allocation is that the very same pointer that is used to queue data is efficiently used to dequeue it and due to its modulo nature, the dequeueing pointer automatically terminates at the point of insertion of the next sample. On a PIC chip, the buffer that was used is illustrated in Figure 3.3.10. Oldest sample is written over the newest sample and File Select Register (FSR) is the hardware pointer used to load and unload data. The illustrated circular buffer holds four elements - EOB marks end-of-buffer, BOB marks beginning-of-buffer, the numeric values in the figure are RAM locations and the sample buffer occupies memory locations from $0 \times 41$ to $0 \times 44$.

EOB

\begin{tabular}{|c|c|c|c|c|c|c|c|c|}
\hline $0 \times 38$ & & & $0 \times 4$ & & & & & $0 \times 15$ \\
\hline $0 \times 38$ & $0 \times 39$ & $0 \times 40$ & $0 \times 41$ & $0 \times 42$ & $0 \times 43$ & $0 \times 44$ & $0 \times 44$ & $0 \times 45$ \\
\hline
\end{tabular}

Figure 3.3.10 Circular buffer: Used to store ADC values for FIR filter

While loading the buffer the pointer FSR could be at any location within the buffer, so before the sample is stored, it is crucial to first check if the pointer has reached EOB. If it is the very first sample then the received data is placed at the $B O B$ or location $0 \times 44$ and the pointer is post-decremented to location $0 \times 43$. In PIC18 assembly, the hardware pointer FSR can load data and post-decrement in a single cycle by using the POSTDEC register. There is no post-increment feature to the hardware pointer system; hence, the $\mathrm{BOB}$ is at a higher memory location than the EOB. When the FSR pointer has been reached, the EOB is simply reset to BOB. Based on this concept, the newest data sample automatically replaces the oldest data sample. 
Illustrated in Figure 3.3.11 is a flowchart showing the process of loading the circular buffer as well as the assembly code written to achieve it.

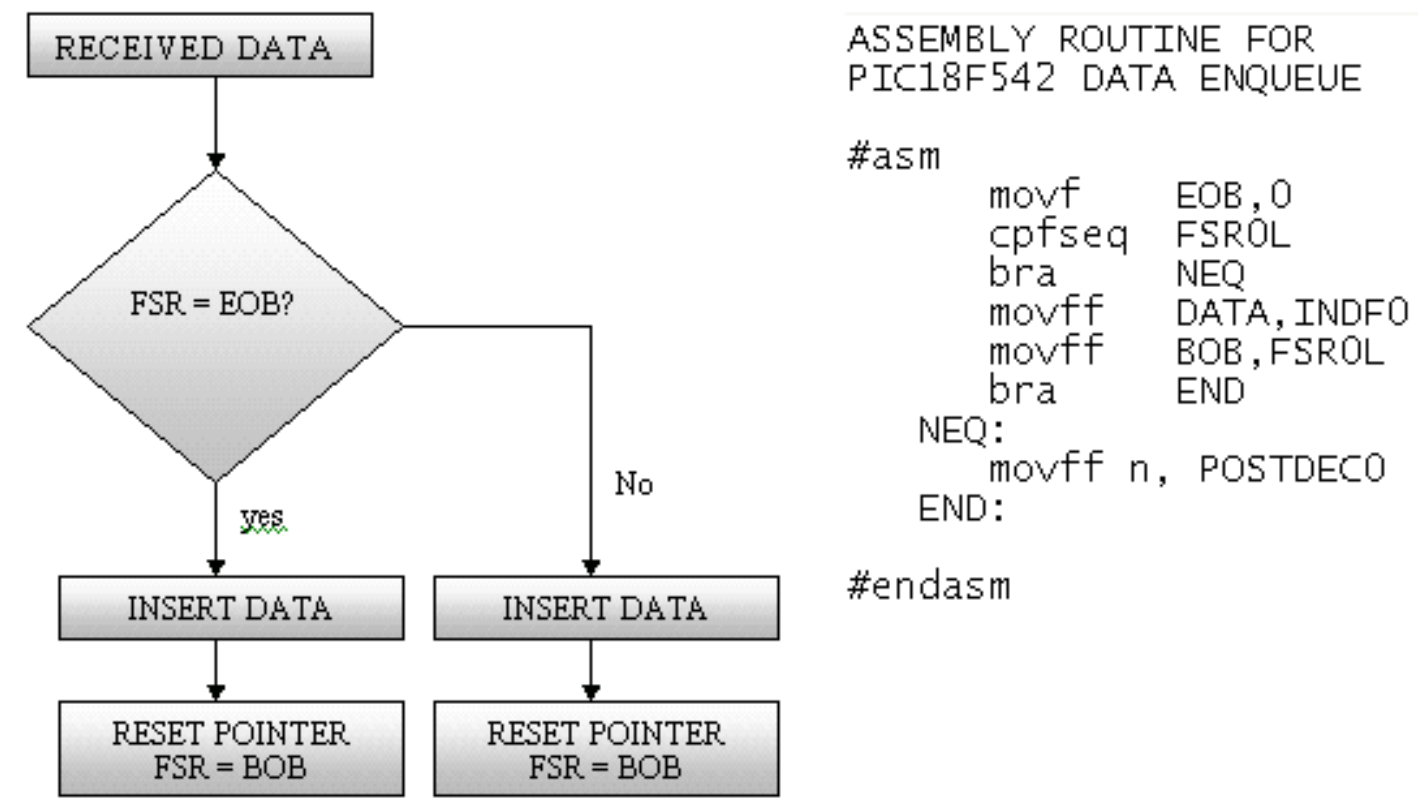

Figure 3.3.11 Algorithm developed to load circular buffer

If the ADC was capturing a ramp in the form of digital data ranging from 0 to 6 then the buffer would load in the following way: The first data point ' 0 ' will be stored at the $\mathrm{BOB}$ and the pointer is decremented as illustrated in Figure 3.3.12. The next sampled data point ' 1 ' is stored in the location pointed by data pointer FSR and the pointer is decremented as shown in Figure 3.3.13.

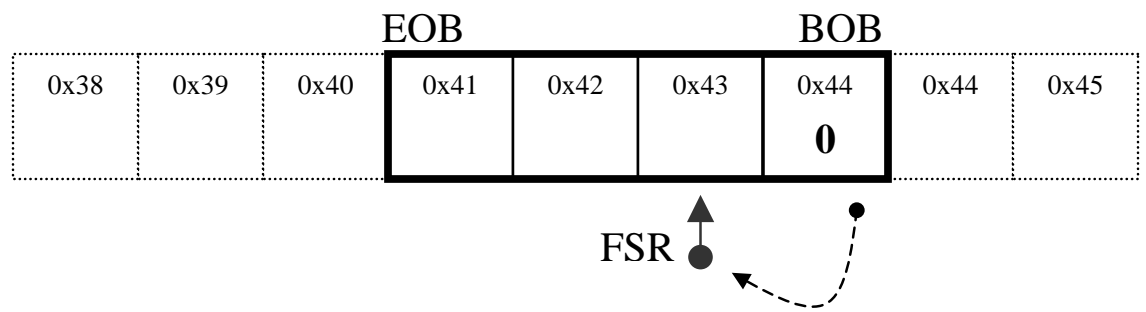

Figure 3.3.12 Step 1: Data element 0 is loaded and pointer decrements 


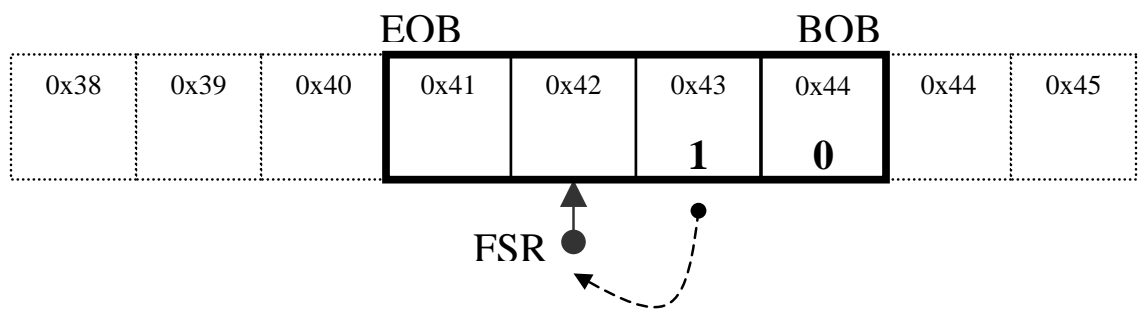

Figure 3.3.13 Step 2: Data element 1 is loaded and pointer decrements

By the time ' 3 ' is sampled the buffer is full and EOB is reached as shown by the illustration. ' 3 ' is stored at EOB and the pointer is reset to the BOB. Now notice the pointer is at the oldest element as shown in Figure 3.3.14.

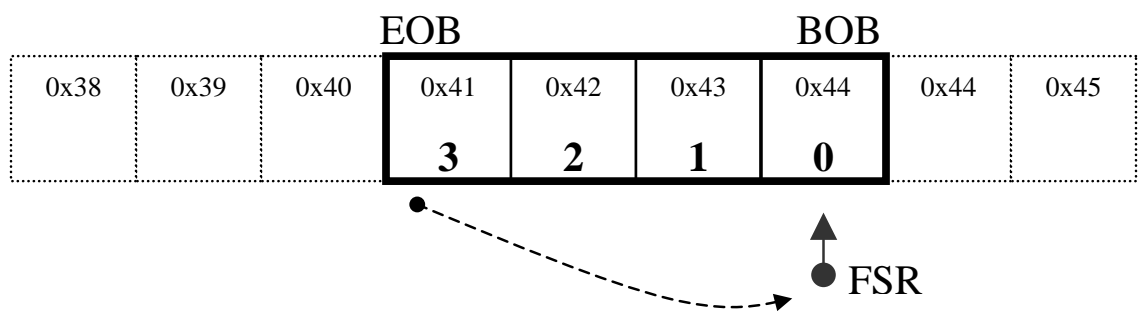

Figure 3.3.14 Step 3: Data element 3 is loaded and EOB is reached

When ' 4 ' is captured it replaces the oldest element in the buffer and the pointer FSR is incremented as normal as shown in Figure 3.3.15.

\begin{tabular}{|c|c|c|c|c|c|c|c|c|}
\hline & & \multicolumn{4}{|c|}{ EOB } & \multicolumn{3}{|c|}{$\mathrm{BOB}$} \\
\hline $0 \times 38$ & $0 \times 39$ & $0 \times 40$ & $\begin{array}{c}0 \times 41 \\
3\end{array}$ & $\begin{array}{c}0 \times 42 \\
2\end{array}$ & $0 \times 43$ & $\begin{array}{c}0 \times 44 \\
4\end{array}$ & $0 \times 44$ & $0 \times 45$ \\
\hline
\end{tabular}

Figure 3.3.15 Step 4: Element 4 is loaded and pointer is pre-decremented 
In order to pull data from the buffer, the pointer FSR would simply travel in the opposite direction and data will be obtained in the exact opposite order to which it had entered. Before each the pointer is advanced it must first check for the BOB or it will travel beyond the buffer. If $\mathrm{BOB}$ is reached the pointer is relocated to EOB. In order to extract data the pre-increment function of the pointer is used so data is pulled and pointer is advanced in a single-cycle. To illustrate the process the pointer is pre-incremented to $0 \times 44$ and ' 4 ' is pulled as illustrated in Figure 3.3.16.

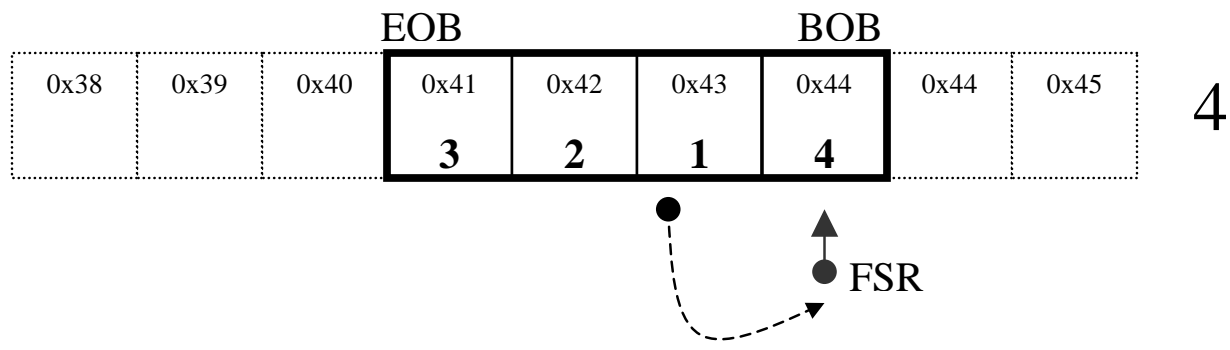

Figure 3.3.16 Element 4 is unloaded from buffer and BOB is reached

Note the pointer FSR is at the beginning-of-buffer so it is first relocated to the $\mathrm{EOB}$ and then the data pulled is ' 3 ' as illustrated in Figure 3.3.17.

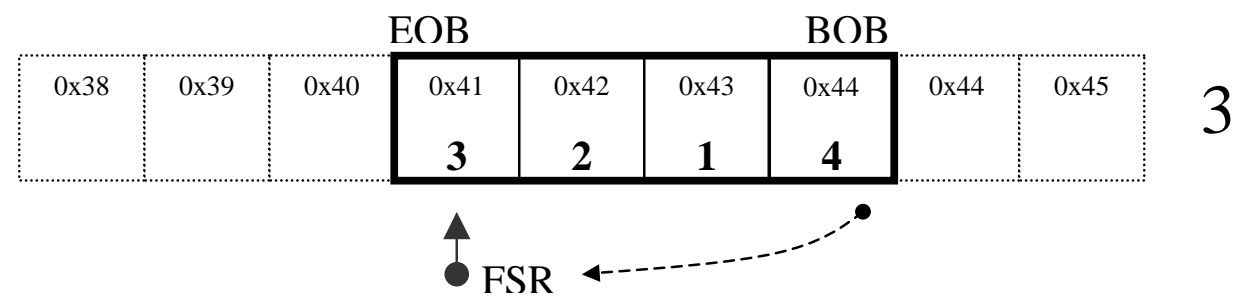

Figure 3.3.17 Pointer is relocated to EOB and 3 is pulled

Pointer is pre-incremented to $0 \times 42$ and ' 2 ' is pulled followed by ' 1 ' and after four iterations the pointer FSR has automatically terminated at 
the entry point where the next incoming data sample is to be placed as shown in Figure 3.3.18.

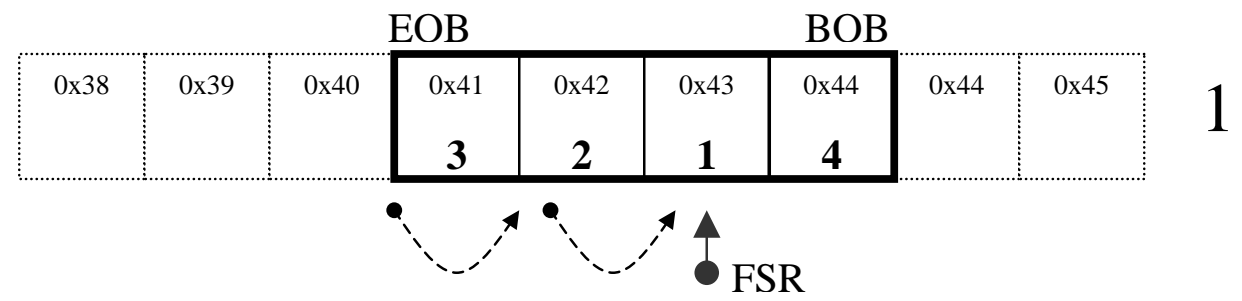

Figure 3.3.18 Data element 1 is pulled

Data went into the buffer in the order $\{1,2,3,4\}$ and came back out $\{4,3,2,1\}$. The formal algorithm and assembly code is in Figure 3.3.19.

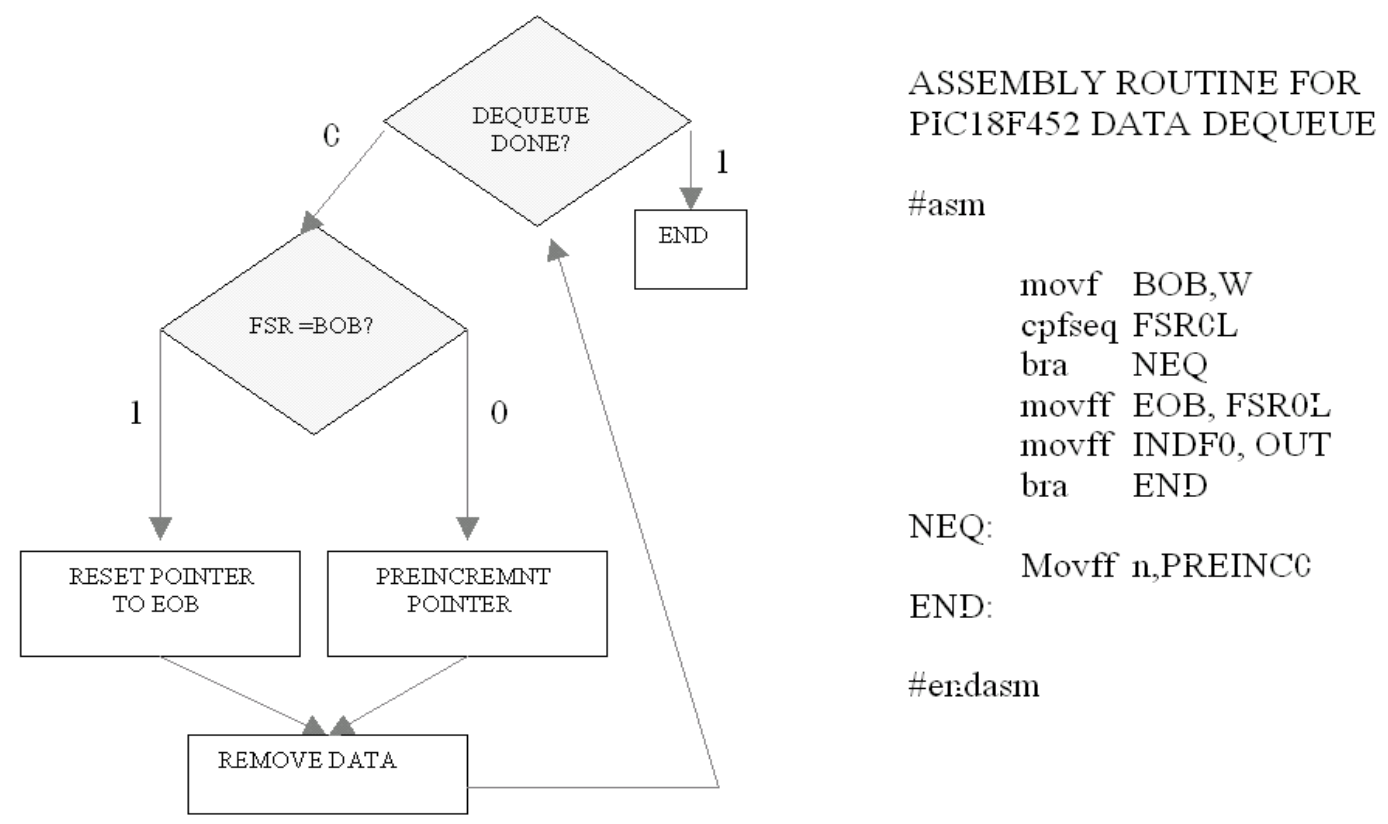

Figure 3.3.19 Algorithm used to pull data from the circular buffer

2. Double circular buffer implementation on PIC 18F452: In the second buffering technique two adjacent circular buffers are used in such a way that the second one begins exactly where the first one ends. Every time a fresh sample is made, it is placed in both buffers in place of the 
oldest sample respectively. Each buffer will have it's own pointer and both buffers will contain the exact data at any given time. [8]

This buffering scheme has a very useful advantage over the previous one because the unloading pointer does no longer need to check for the end of buffer (EOB). Figure 3.3.20 of the buffering scheme might explain the process more clearly.

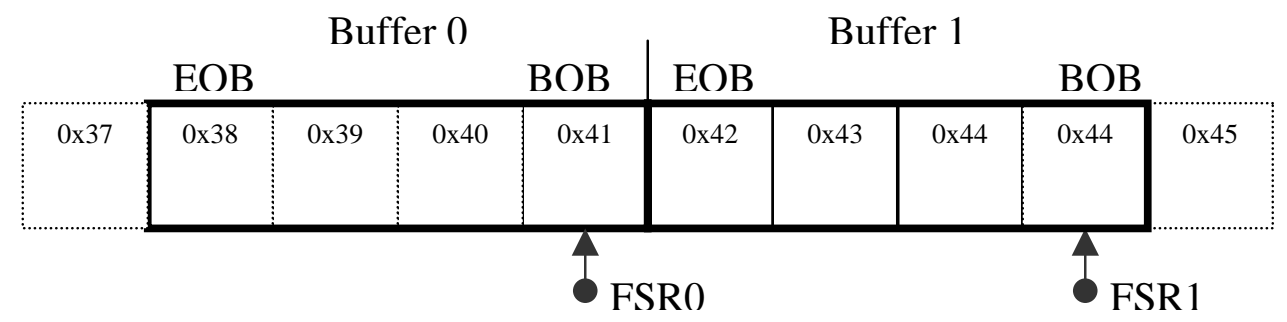

Figure 3.3.20 Topology of the Double Circular Buffers

Once again the same data is being stored in the buffer, each data element is stored in the same respective place in both buffers. If the first sample element is ' 1 ' then both buffers will store the data and postdecrement in the same manner as if each was an independent buffer.

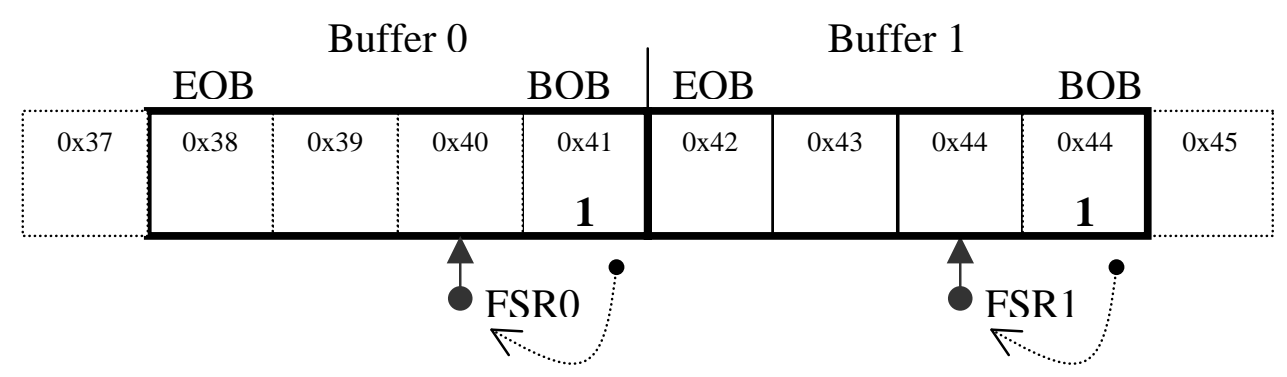

Figure 3.3.21 Data Element 1 is loaded to both buffers

Since both pointers move in tandem, only one needs to be checked for $\mathrm{EOB}$ and although this technique takes a little more time to load, it saves a lot more time during the unload. Since FIR filtering involves only 
a single load and $\mathrm{N}$ number of unloads ( $\mathrm{N}$ being the number of coefficients), over all this technique produces a tremendous savings in computation time for each FIR output calculation.

After ' 1 ' the next data sample is ' 2 ' then ' 3 ' then ' 4 ' followed by ' 5 ' and the buffer will fill in the manner illustrated in Figure 3.3.22.
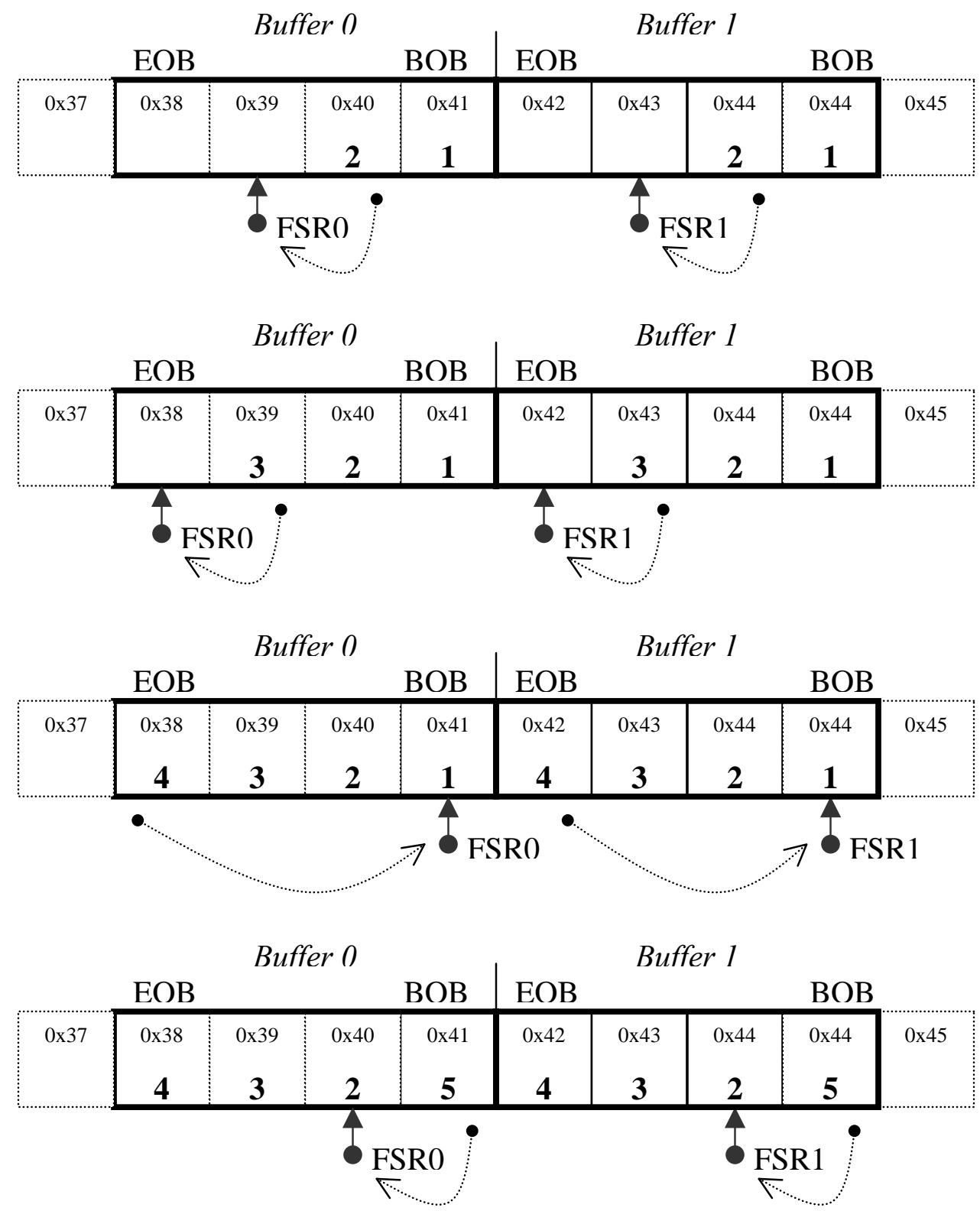

Figure 3.3.22 Data Elements 1-5 are loaded to buffer 
Unloading data from the buffer involves a slightly different technique than what is used for a single buffer. Since the FIR filtering algorithm involves a convolution operation, after every fresh sample is stored the filter needs to unload each data point in reverse chronological order to perform computation. Since the size of the buffer is known, say $N$, there is no need to test of end-of-buffer or beginning-of-buffer while pulling the data because pointer FSR1 can now simply cross over from its own buffer into the adjacent one and always find the chronologically correct sample, sitting beyond the barrier of the adjoining buffer. To illustrate the point say we wanted to pull data from the current buffer. The last data sample stored was ' 5 ' as shown in Figure 3.3.23.

\begin{tabular}{|c|c|c|c|c|c|c|c|c|c|}
\hline & \multicolumn{4}{|c|}{ Buffer 0} & \multicolumn{4}{|c|}{ Buffer 1} & \\
\hline & EOB & & & $\mathrm{BOB}$ & EOB & & & $\mathrm{BOB}$ & \\
\hline $0 \times 37$ & $0 \times 38$ & $0 \times 39$ & $0 \times 40$ & $0 \times 41$ & $0 \times 42$ & $0 \times 43$ & $0 \times 44$ & $0 \times 44$ & $0 \times 45$ \\
\hline & 4 & 3 & 2 & 5 & 4 & 3 & 2 & 5 & \\
\hline
\end{tabular}

Figure 3.3.23 Buffer Data Ready to be unloaded

Data can be pulled in ascending order or descending order depending on which of the two pointers are used. For FIR filter calculations the order of the sample is not important because the coefficients are symmetric. If descending order were desired we would first relocate FSR1 to the same location as FSR0 as shown in Figure 3.3.24.

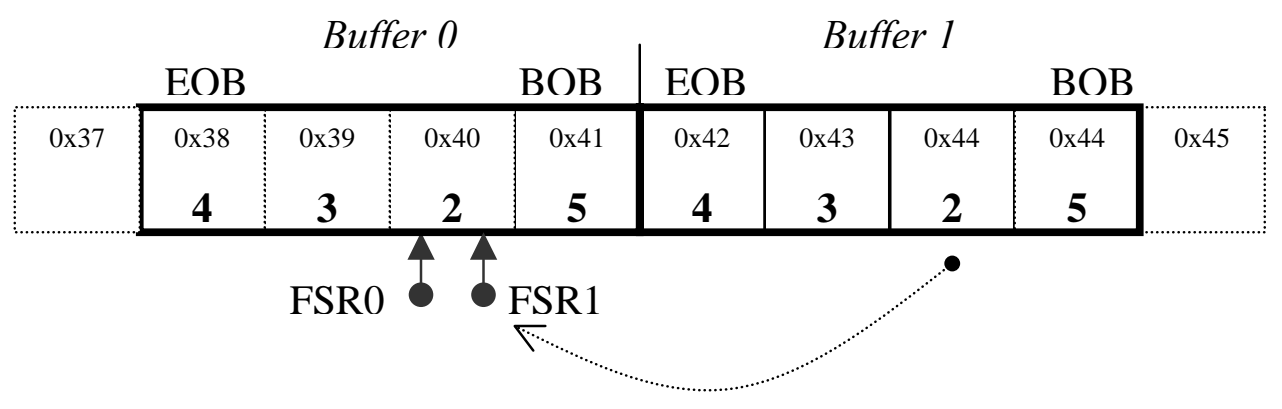

Figure 3.3.24 Pointer FSR1 is relocated to same location as FSR0 
All that remains now is to pre-increment the pointer and pull the respective data sample from each location the pointer passes till it returns to the position it started (location $0 \times 44$ ).

The first data sample to be removed is ' 5 ' as the pointer FSR1 preincrements from location $0 \times 40$ to $0 \times 41$, both operation in one cycle as shown in Figure 3.3.25.

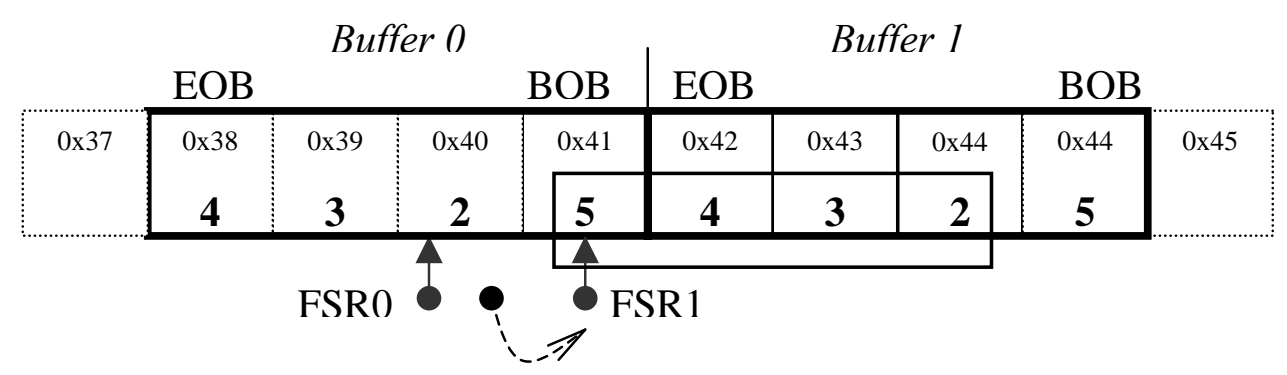

Figure 3.3.25 FSR1 pre-increments and unloads data element 5

Notice that data samples '5','4','3' and '2' are in chronological order across both buffers. Since the buffer size is a constant, 4 , then four blind pre-increment operations will unload the buffer and the pointer will automatically be returned to the point of insertion of the next sample. Using this method neither EOB nor BOB needs to be checked while unloading the buffer. 
The Figure 3.3.26 shows the flow-chart describing the algorithm for loading the adjacent circular buffers and Figure 3.3.27 shows the assembly language code written to implemented it.

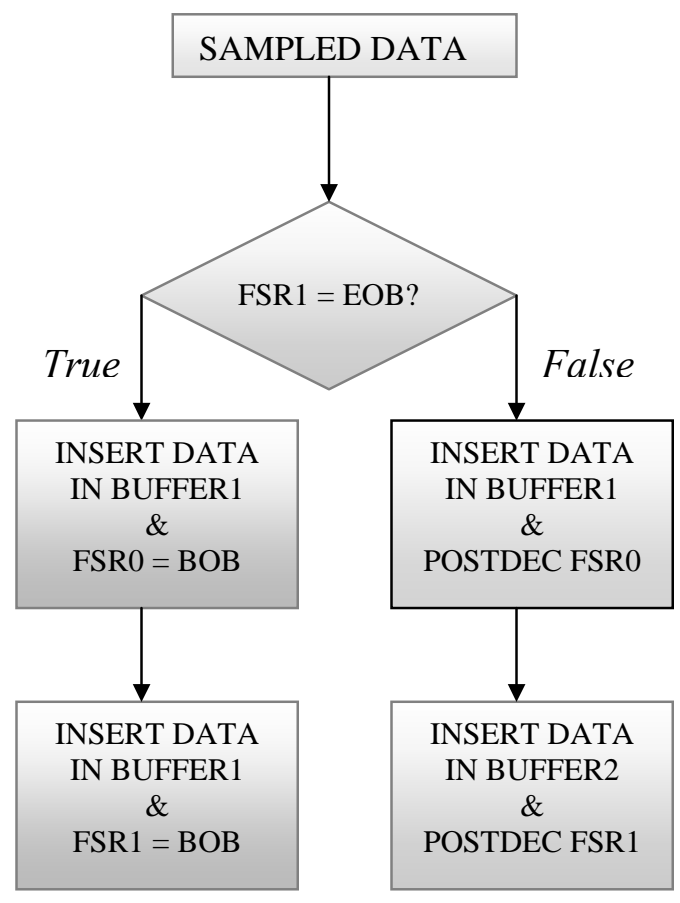

Figure 3.3.26 Algorithm for loading the adjacent circular buffers

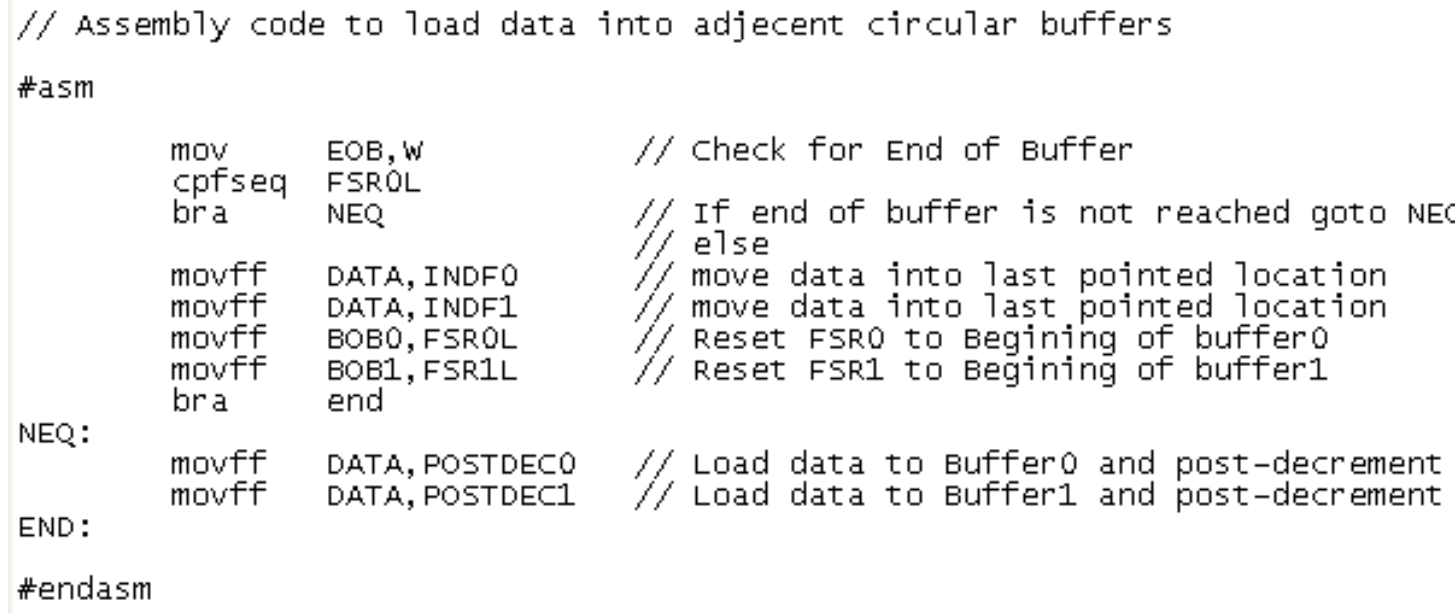

Figure 3.3.27 Assembly routine written to load the buffers 
The flowchart outlines the algorithm for pulling data from the buffers is shown in Figure 3.3.28:

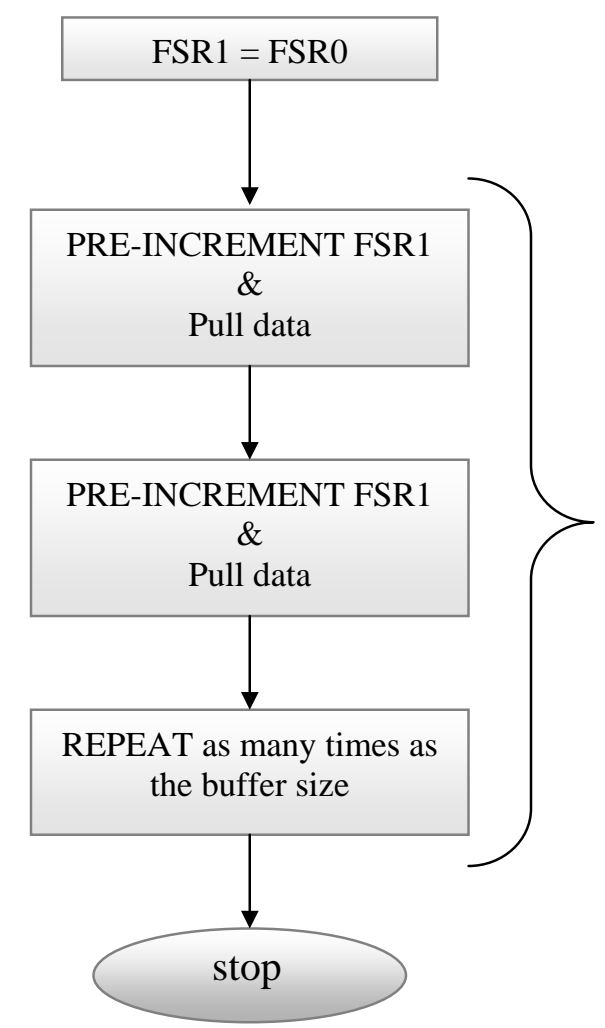

Figure 3.3.28 Algorithm used to unload from adjacent circular buffers

\subsubsection{Sampling: Analog to Digital Conversion on 18F452}

The most convenient option for analog to digital conversion is by using the integrated ADC module. The built in analog to digital converter uses a successive approximation algorithm and is capable of converting an analog voltage into a proportional 10-bit number. The ADC is capable of a maximum sampling rate of $52 \mathrm{KHz}$ for $10 \mathrm{Bit}$ conversions. For 8-Bit conversions, the maximum sampling rate is $62.5 \mathrm{KHz}$ at same temperature and impendence [10]. 
The value sampled by the ADC is stored in the register pair ADRESH/ADRESL. Each is 8 bits, ADRESH contains the high-byte and ADRESL holds the low-byte. In order to configure the ADC module the ADC control register pair ADCON0 and ADCON1 must be set with appropriate values.

The analog-to-digital converter module has eight input channels for the PIC 18F452. Each input is a separate channel multiplexed with a common converter. This allows sampling of several different sources in any specified order. Since there is only one analog-to-digital converter simultaneous sampling is not possible using the internal $A D C$ module. The minimum wait time between the sampling of any two channels is called acquisition time. The acquisition time is a function of the ambient temperature and the source impedance. The maximum recommended source impedance or input impedance for analog sources is $2.5 \mathrm{~K}$. For the FIR filter only one channel is needed but for the adaptive filter two channels must be sampled nearly simultaneously.

The first task to setting up the ADC is to setup the control register pair ADCON0 and ADCON1. Both registers are eight bits wide and allow unrestricted read/write operations. The ADCON0 register controls ADC clock options, channel selection, and the bit GO/DONE in the ADCON0 register can be polled in order to check if analog to digital conversion is complete. The ADCON1 register controls the remaining clock options, shared with ADCONO and selects which pins are configured as digital and which are analog. In order to setup the internal ADC both registers must be loaded with the appropriate values. 
Configuring ADCON0 involves setting five bits on the register. The following is the contents of the ADCON0 Register shown in Figure 3.3.29.

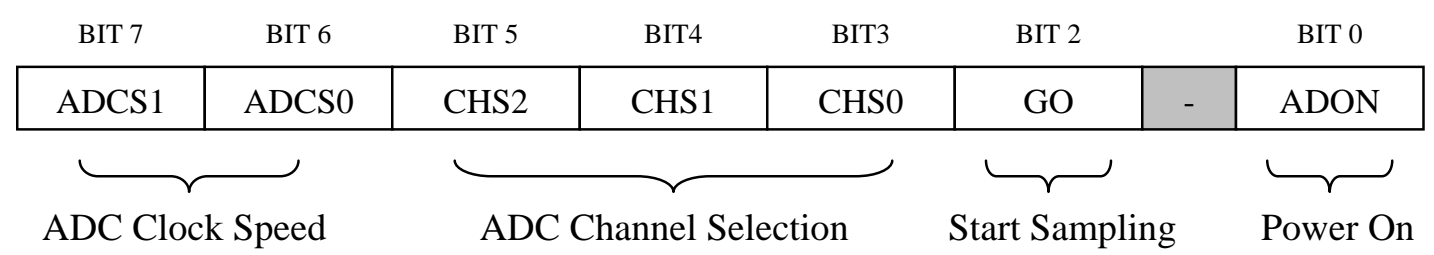

Figure 3.3.29 Description of the ADCON0 Register

The ADC clock is derived from the main external oscillator. The PIC chip can run up to $40 \mathrm{Mhz}$ but the ADC clock cannot exceed $625 \mathrm{KHz}$. Therefore the PIC must use a clock divide to scale the $40 \mathrm{MHz}$ external frequency to $625 \mathrm{KHz}$, a factor of 64 . Hence the ADCS1 and ADCS0 are 1, 0 to make the clock divider equal to 64. The channel for FIR filter is channel-0 hence the CHS2, CHS1, CHSO are 0,0,0 and ADON is 1.

\section{$\operatorname{ADCON0}=<\begin{array}{lllllll}10000 & 0 & 0 & 1\end{array}$ or $0 \times 85$}

The ADCON1, shown in Figure 3.3.30, is set in a similar manner as ADCON0 and it contains:

\begin{tabular}{|c|c|c|c|c|c|c|c|}
\hline BIT 7 & BIT 6 & BIT 5 & BIT4 & BIT3 & BIT 2 & BIT 1 & BIT 0 \\
\hline ADFM & ADCS2 & - & - & PCFG3 & PCFG2 & PCFG1 & ADON \\
\hline
\end{tabular}

Figure 3.3.30 Description of the ADCON1 Register

The ADC stores a 10 -bit result in two 8-bit registers. The ADFM bit selects if the result is left justified or right justified. Since the FIR filter 
is going to use 8-bit samples instead of 10-bit samples, ADFM will be set to 0 to make the result left justified. A simple way to get a fast 8-bit approximation of the 10 -bit sample is to only read the $A D R E S H$ register as illustrated in Figure 3.3.31.

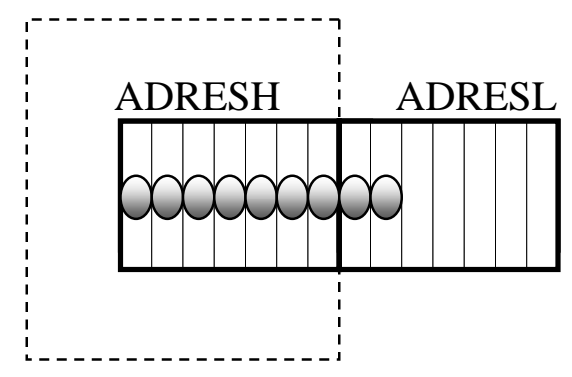

Figure 3.3.31 Reading Only ADRESH will scale down to 8-bit

The ADCS2 bit is set to 1 to make the clock divide equal to 64 as discussed before. Bits <PCFG3, PCFG2, PCFG1, PCFG0> are set to 1,1,1,0 respectively. This allows pins A0 to be analog while all other pins are made digital. Since technically only a single analog pin is required to make a FIR filter. If more analog pins are needed then this register needs to be changed. The ADCON1 register is loaded with the following:

\section{$\operatorname{ADCON} 1=<\begin{array}{lllllll}0 & 0 & 0 & 1 & 1 & 0 & \text { or } 0 \times 4 E\end{array}$}

$\mathrm{V}_{\mathrm{DD}}$ and $\mathrm{V}_{\mathrm{SS}}$ are used as voltage references with this configuration.

The FIR filters performance depends not only on sampling signals accurately but also on a chips ability to maintain a constant sampling rate. To this end, one of the PIC chips three hardware timers; timer1 is dedicated to performing analog to digital conversion at a periodic rate. This is a 16-bit timer that derives its timing from the external clock source and interrupts the PIC chip when it overflows. Once the timer is engaged it counts from 0 till 65535 at the what ever speed it been clocked and at the end of its count generates an interrupt. 
In order to make a constant sampling rate the timer is not allowed to start from 0 but instead made to start from some offset value from which it will pass 65535 at a predictable interval since the clock speed to the timer is known. This offset value is calculated using the following way:

$$
\text { timer1_offset }=65535-\frac{\text { external_osc }}{4 * \text { prescaler } * \text { sampling_rate }}
$$

In this case, the external oscillator (external-osc) is $40 \mathrm{MHz}$ and the user determines the sampling rate in the design stage.

The interrupt service routine for timer1 will also perform all the calculations required of the filter and before exiting the Interrupt Service Routine (ISR) the result of the filter is generated.

\subsubsection{Filter Calculations}

Three different strategies are used to perform the necessary filter calculations.

1. Optimized for maximum Speed

2. Optimized to use minimal Ram

3. Optimized to generate smallest program size.

All three strategies make use of the same general idea but are different in the way the data is buffered and computation is performed. In general the realization of FIR filters is obtained by the direct computation of the Equation 3.3 [12].

$$
y_{n}=\sum_{0}^{N} x[n-N+1] *\left[K_{N-1}+128\right]-\sum_{0}^{N} x[n-N+1] * 128
$$


The equation presented above is a variation of the classical FIR filter equation that is presented in most books:

$$
y_{n}=\sum_{0}^{N} x[n-N+1] * k_{N-1}
$$

In both Equations 3.3 and 3.4, the term $y_{n}$ is the output of the filter and is computed by the linear convolution of the coefficient matrix $K_{N}$ and the discrete sample vector $\chi_{n}$. Both equations perform exactly the same computation and produce the same results however Equation 3.3 is far more PIC18F452 architecture-friendly because the signed multiplication operation in Equation 3.4 has been removed. This will allow PIC to maximize the use of the unsigned hardware-multiplier in the PIC hardware.

The only difference between Equation 3.4 and Equation 3.3 is that in Equation 3.3 the tap co-efficient vector $K_{N}$, which contains signed numbers ranging from -128 to +128 are made unsigned by adding to them the integer 128. In order to balance the result from the offset coefficients it becomes necessary to subtract $128 * \Sigma x_{n}$ from $y_{n}$. To illustrate this point, consider the following analogy. If we wanted to calculate the $A$, which is a product between 8 -Bit signed integer $B$ and 8 Bit unsigned integer $C$, it will be given by:

$$
\mathrm{A}=\mathrm{B} * \mathrm{C}
$$

The above computation will require a signed multiplication however if we modified the above equation in the following manner:

$$
\mathrm{E}=\mathrm{B} *(\mathrm{C}+128)-\mathrm{B} * 128
$$


then we ultimately achieve the very same result as $A$ and avoid the signed multiplication altogether.

$$
\mathrm{A}=\mathrm{E}
$$

\subsubsection{Implementation for Shortest Execution Time}

As stated before, three different implementations are possible using the filter design system. The first has the shortest possible execution time and possibly the most attractive implementation of all. The short execution time is achieved at the expense of higher RAM usage, since two adjacent circular buffers are used to store ADC samples instead of one. This doubles RAM use and also produces a much larger program, because to fully make use of the double buffer, the main multiply-accumulate loop is unrolled allowing for program to approach its theoretical minimal computation time, given the data word length constraints that is used by the program [12].

The implementation is split into two routines. The first one is the initialization Routine and the second is the Computation Routine. The initialization routine runs just once when the program begins and it serves only to initialize the buffers and other variables that are required for FIR filter calculations. The computation routine performs all calculation mandated by the filter and runs inside the interrupt service routine of timer1. With the confinement of all filter calculations inside the ISR, we achieve a degree of isolation making it possible for any main application to use the filter and not interfere with its operation or timing.

Memory Usage: The PIC 18 F452 chip contains 1536 bytes of RAM and two addressing modes. There is direct-addressing and indirectaddressing. Indirect-addressing uses three pointers - FSR0, FSR1, FSR2 
and each pointer is 12 -Bit wide, with a 4 -Bit select bank and 8 -Bit select location within a bank. All memory use for the FIR filter is restricted to a single bank, thereby limiting the available memory for ADC samples and filter coefficients to a total of 256 Bytes. The obvious advantage of limiting all pointers use to a single bank is the speed that is gained because the pointers can be used faster if the bank does not need to be set before every call. Three buffers are used - a static buffer for tap coefficients that is loaded and initialized at start up and two identical adjacent circular buffers for the incoming ADC samples. All three buffers are of the same size and each buffer is given its own hardware pointer. Consider Figure 3.3.32 showing memory footprint

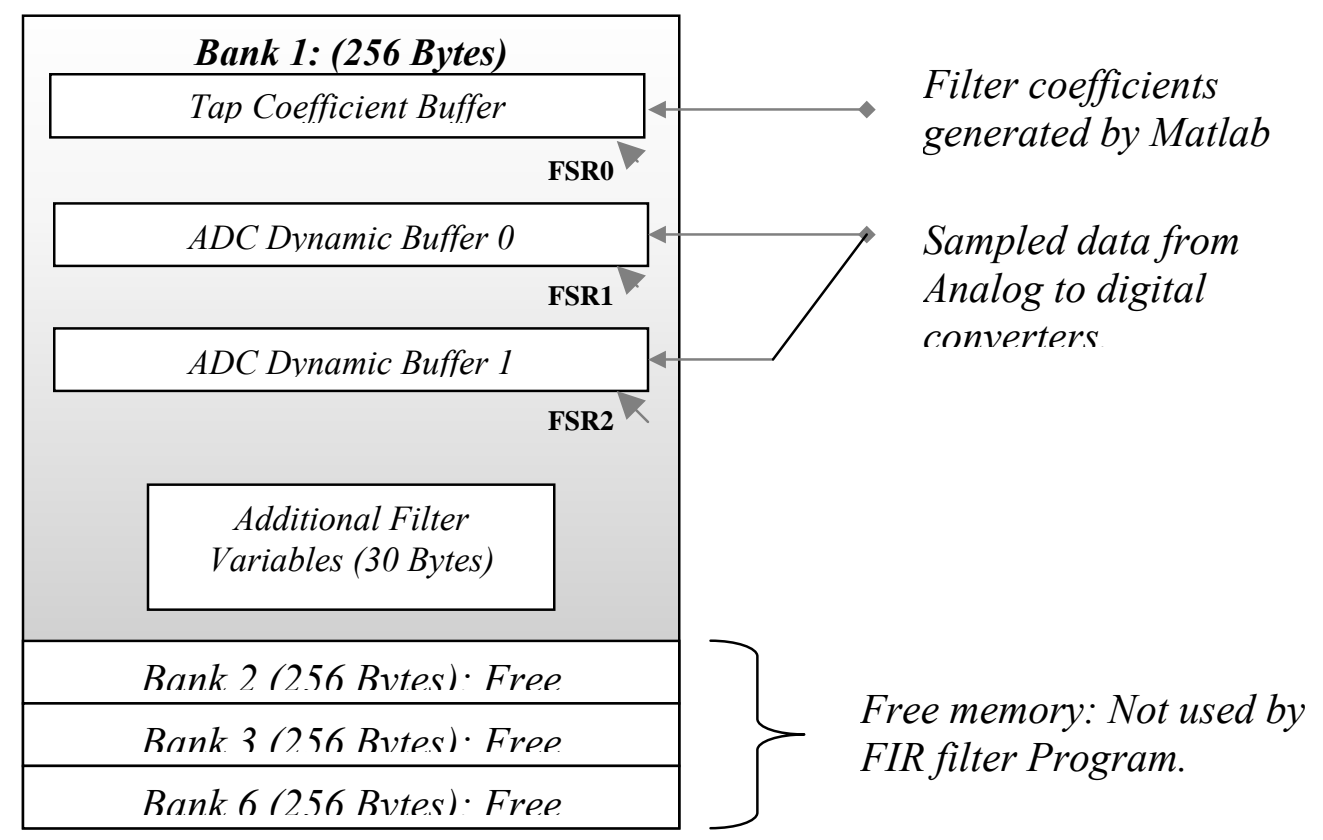

Figure 3.3.32 RAM used by the FIR filtering scheme

The buffers for the tap coefficients and ADC values can be of variable size, since the number of FIR filter coefficients is not fixed. The total memory allocated to the buffers cannot exceed 226 bytes. No buffer can be larger than 75 elements. Hence this design does not allow for FIR filters larger than 75 taps. The MATLAB user interface will generate a 
warning if the user specifies a filter that generates more than 75 coefficients and the user is prompted to either accommodate fewer taps or select a different implementation strategy.

\section{Filter Processing: (Initialization Routine)}

If the filter is to produce an output, then an output port needs to be assigned, the first step of the initialization routine being the setup of an I/O port. Analog to digital converter is set to 8-Bit mode by simply leftshifting the results and reading the ADRESH register (See 3.3.6).

The timer1 interrupt must be set to sample at the user specified sampling rate. All filter processing is conducted in the timer interrupt. There are three registers associated with Timer1. These are T1CON, TMR1H, and TMR2L. The first is the control register the other two are offset registers that are used to initialize the timer. Timer1 can be setup as an 8 -bit as well as 16-bit as shown in Figure 3.3.33.

\begin{tabular}{|c|c|c|c|c|c|c|c|}
\hline BIT 7 & BIT 6 & BIT 5 & BIT4 & BIT3 & BIT 2 & BIT 1 & BIT 0 \\
\hline RD16 & - & T1CKPS1 & T1CKPS0 & T1OSCN & T1SYNC & TMR1CS & TMR1ON \\
\hline$r$ & & & & $\underbrace{r}$ & $\underbrace{r}$ & $\underbrace{r}$ & $\underbrace{r}$ \\
\hline $\begin{array}{c}16 / 8 \text { mode } \\
\text { select }\end{array}$ & & imer1 Input & e-scale Bits & $\begin{array}{l}\text { Oscillator } \\
\text { input bit }\end{array}$ & $\begin{array}{c}\text { External } \\
\text { Clock Sync }\end{array}$ & $\begin{array}{l}\text { Edge } \\
\text { Select }\end{array}$ & On/Off \\
\hline
\end{tabular}

Figure 3.3.33 Description of the T1CON Register

In order to setup the timer bit RD16 $=1$, T1CKPSX is calculated in Matlab and set based on user specified sampling rate. T1OSCEN, T1SYNC are not used, TMR1CS $=0$ to specify internal clock and TMR $1 \mathrm{ON}$ is 1 to power on the timer.

The timer offset is calculated using equation discussed in section 3.3.6. The 16-Bit offset is loaded into register pair TMR1L, TMR1H. All 
the registers are automatically loaded by code generated by the MATLAB program based on the sampling frequency selected by the user.

Before the timer is engaged the initialization routine loads the coefficients into the coefficient buffers and both the ADC buffers are set up, thereby initializing the pointers. Pointer FSR0 is used to load the coefficient buffer and pointers FSR1, FSR2 are used to address the ADC buffers. ADC buffering method is discussed in detail in section 3.3.5. Once all the buffers are initialized the timer1 is started.

\section{Filter Processing: (Computation Routine)}

The computation routine involves the real-time implementation of the FIR filtering algorithm given in Equation 3.8.

$$
\underbrace{y_{n}=\sum_{0}^{N} x[n-N+1] *\left[K_{N-1}+128\right]}_{\text {Multiply \& Accumulate }\left(Y_{1}\right)} \underbrace{-\sum_{0}^{N} x[n-N+1]}_{\text {Accumulate }(\Sigma \mathrm{x})} * 128
$$

In order to compute filter output Yn or Equation 3.8 is broken into three different Equations 3.9, 3.10 and 3.11.

$$
\begin{gathered}
y_{1} \underbrace{\sum_{0}^{N} x[n-N}_{\text {Sample vector }} \underbrace{+1] *\left[K_{N-1}\right.}_{\text {Tap Coefficients }}+128] \\
\sum \underbrace{\sum_{0}^{\sum_{0}^{N} x[n-N+1] * 128}}_{\text {Sample Sum }} \\
Y_{n}=Y_{1}-\sum x
\end{gathered}
$$


Equation 3.9 is implemented using a fixed-point multiply-accumulate operation block. The MAC block is repeated for $\mathrm{N}$ times till $y_{1}$ is computed. A 24-Bit register comprised of three 8-bit registers is assigned to hold the MAC result shown in Figure 3.3.34.

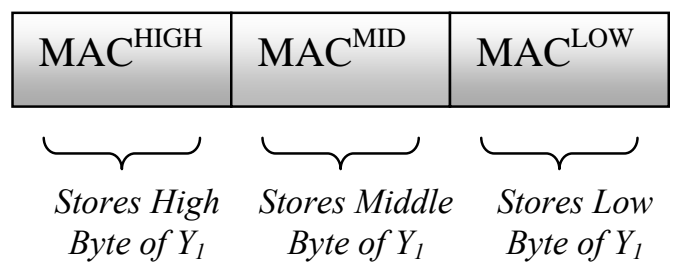

Figure 3.3.34 Word space dedicated to storing MAC result

Equation 3.10 computes the sample-sum of all the samples held in the ADC buffers. $\Sigma_{\mathrm{x}}$ is given a 16 -Bit unsigned variable comprising to register pair $\Sigma_{\mathrm{x}}^{\mathrm{LOW}}$ and $\Sigma_{\mathrm{x}}{ }^{\mathrm{HIGH}}$, shown in Figure 3.3.35. It is calculated with minimal computational effort by simply subtracting from the total, the oldest ADC sample and adding the newest one every time a new sample is made. Thus a running total of all the samples in the buffer is constantly maintained without having to add up every value in the buffer each time a new sample is added to it.

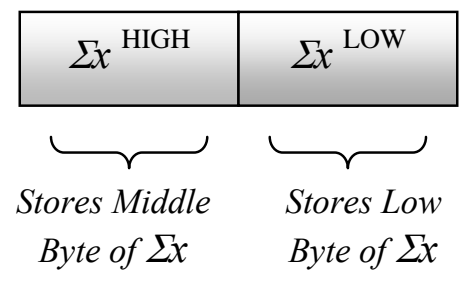

Figure 3.3.35 Word space dedicated to storing Sample Sum

$\Sigma_{\mathrm{X}}$ has to be multiplied by 128 and subtracted from $Y_{1}$ to obtain final output Yn. An efficient way to multiply by 128 is to copy the sum into another 24 -bit variable i.e. moving $\Sigma \mathrm{x}^{\text {LOW }}$ to $255 \sum \mathrm{x}^{\text {MID }}$ and $\Sigma \mathrm{x}^{\text {HIGH }}$ to 
$255 \sum \mathrm{x}^{\mathrm{HIGH}}$ and clearing the $255 \sum \mathrm{x}^{\mathrm{LOW}}$ will do an implicit multiply by 255 as shown in Figure 3.3.36.

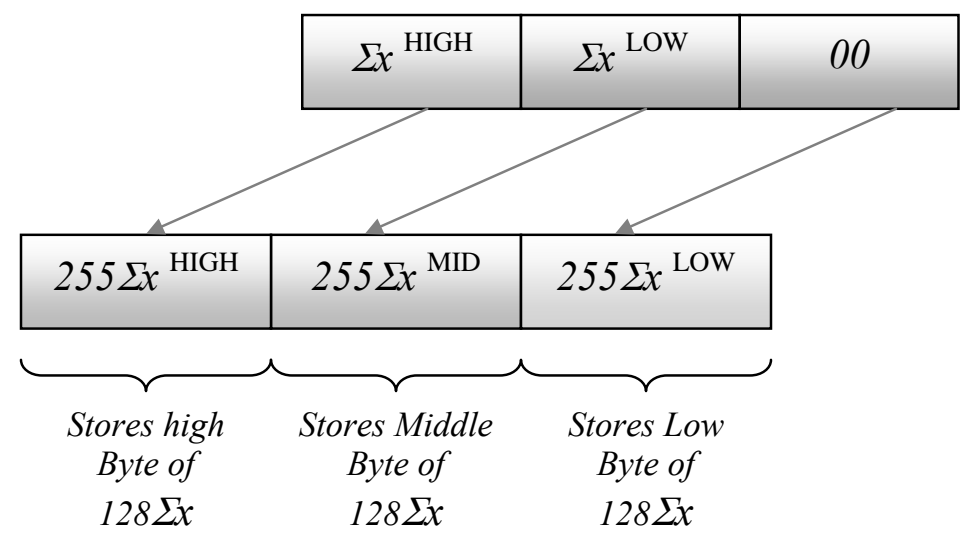

Figure 3.3.36 Multiply by 256 algorithm

Once moved a single right-shift with carry on all three registers produces the required multiply by 128 shown in Figure 3.3.37.

Right Shift each by 1 place to multiply by 128

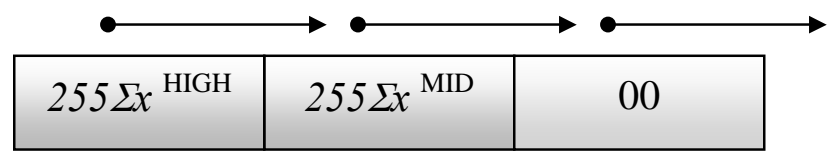

Figure 3.3.37 Multiply by 128: Single Right shift of each byte

The MAC block for computing $\mathrm{Y}_{1}$ uses three buffers that are located in RAM. The Buffers shown here are 4 elements long but can extend up to 75 elements depending on filter requirements. All buffers are dynamically scaled depending on filter requirements. A double buffering scheme is used for storing ADC samples and a single static buffer is used to store filter tap coefficients. FSR0, FSR1 and FSR2 pointers dedicated to each buffer as shown in Figure 3.3.38 and Figure 3.3.39. 


\begin{tabular}{|c|c|c|c|c|c|c|c|c|c|}
\hline & \multicolumn{4}{|c|}{ ADC Buffer 1} & \multicolumn{4}{|c|}{ ADC Buffer 0} & \\
\hline & $\mathrm{EOB}$ & & & $\mathrm{BOB}$ & EOB & & & $\mathrm{BO}$ & \\
\hline $0 \times 37$ & 0x38 & $0 \times 39$ & $0 \times 40$ & $0 \times 41$ & $0 \times 42$ & $0 \times 43$ & $0 \times 44$ & $0 \times 44$ & $0 \times 45$ \\
\hline & 0 & 0 & 0 & 0 & 0 & 0 & 0 & 0 & \\
\hline
\end{tabular}

Figure 3.3.38 Buffer setup for storing ADC samples

Tap Coefficients

\begin{tabular}{|c|c|c|c|c|}
$0 \times 60$ & EOB & \multicolumn{3}{|c}{ BOB } \\
\hline & $\mathbf{0}$ & $\mathbf{0}$ & $\mathbf{0}$ & $\mathbf{0}$ \\
\hline FSR 2 & $0 \times 61$ & $0 \times 63$ & $0 \times 64$ \\
0
\end{tabular}

Figure 3.3.39 Buffer setup for storing Coefficients

The MAC cycle accumulator occupies three bytes of memory to store a 24-bit number as shown in Figure 3.3.40.

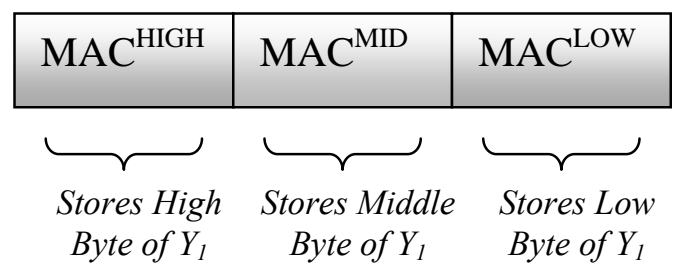

Figure 3.3.40 24-Bit Result store for MAC operation 
Given the above constraints of 8-bit unsigned coefficients, 8-bit ADC samples and 24-Bit accumulator the quickest possible MAC on the PIC 18 F452 is shown in Figure 3.3.41.

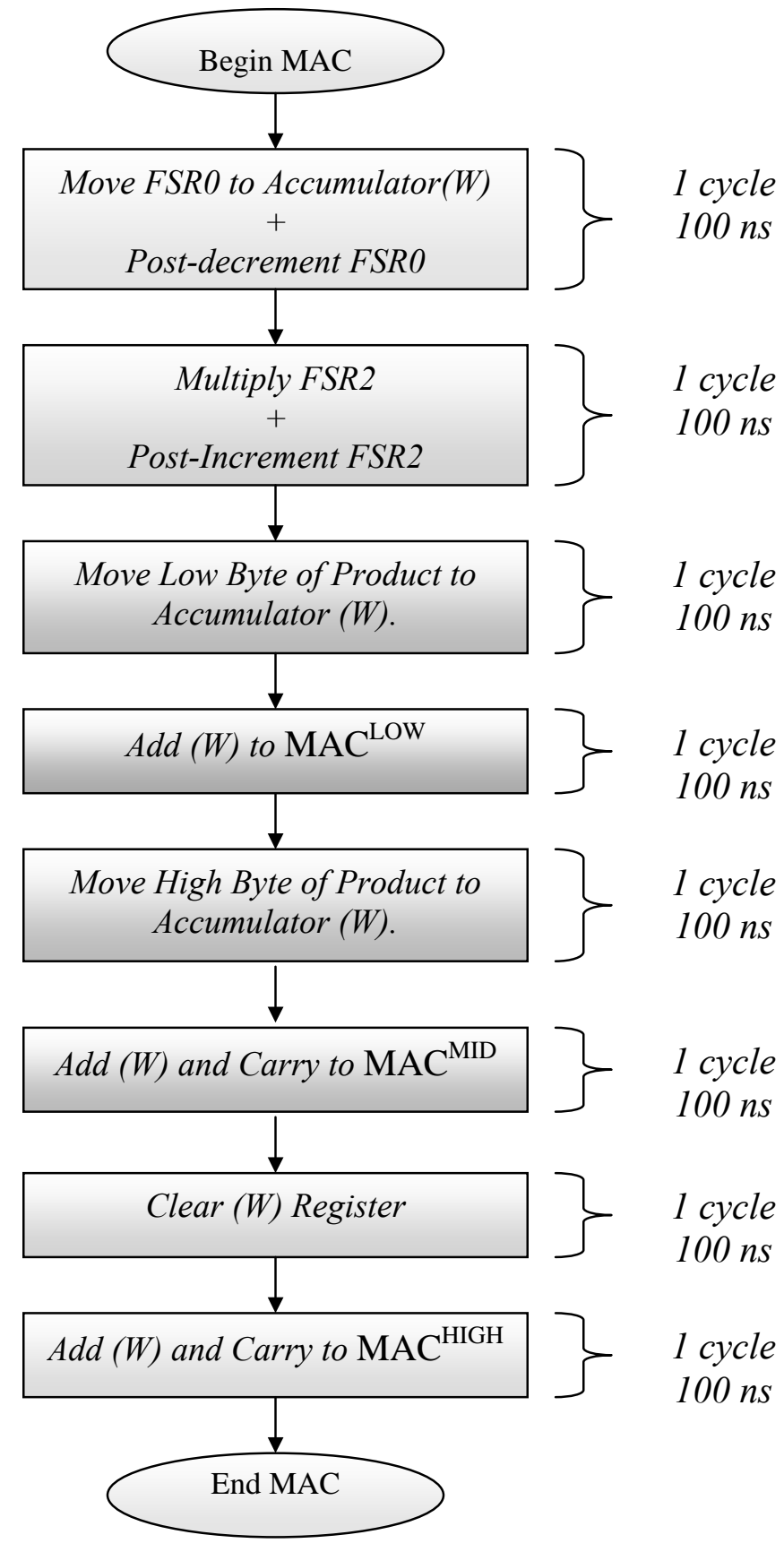

Figure 3.3.41 Multiply-Accumulate Algorithm 
The entire MAC cycle lasts $800 \mathrm{~ns}$ and the assembly code generated for it is as follows:

$\begin{array}{lll}\text { movf POSTDECO,W } & \text { // Move element pointed by FSRO to (W) } \\ \text { mulwf POSTINC2 } & \text { // Multiply FSR2 and Post-increment } \\ \text { movf PRODL,W } & / / \text { Move Product Low-byte to (W) } \\ \text { addwf output_least } & / / \text { Add (W) to the MAC LOW } \\ \text { movf PRODH,W } & / / \text { Move Product High-Byte to (W) } \\ \text { addwfc output_middle } & / / \text { Add carry }+(W)+M A C^{M I D} \\ \text { clrf WREG } & / / \text { Clear }(W) \\ \text { addwfc output most } & / / \text { Add carry }+(W)+M A C^{H I G H}\end{array}$

In order to complete the implementation of Equation 3.10 the pointer FSR2 is first moved to the same location as FSR1 then the MAC block is repeated as many times as the filter order. This way there is no need to check for end-of-buffer or the beginning-of-buffer and final MAC block terminates with the pointer automatically returned to the exact point of insertion of the next incoming sample. Figure 3.3.42 is a flow diagram for the initialization routine for a 4-tap FIR filter.

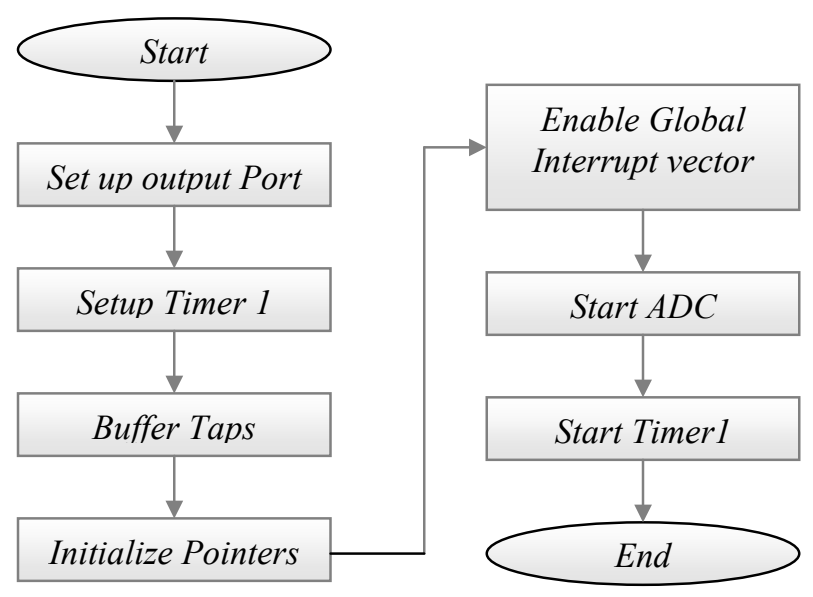

Figure 3.3.42 Initialization Routine for Fastest Execution 
Figure 3.3.43 is a flow diagram for the computation routine the fastest execution time version of a 4 tap FIR filter.

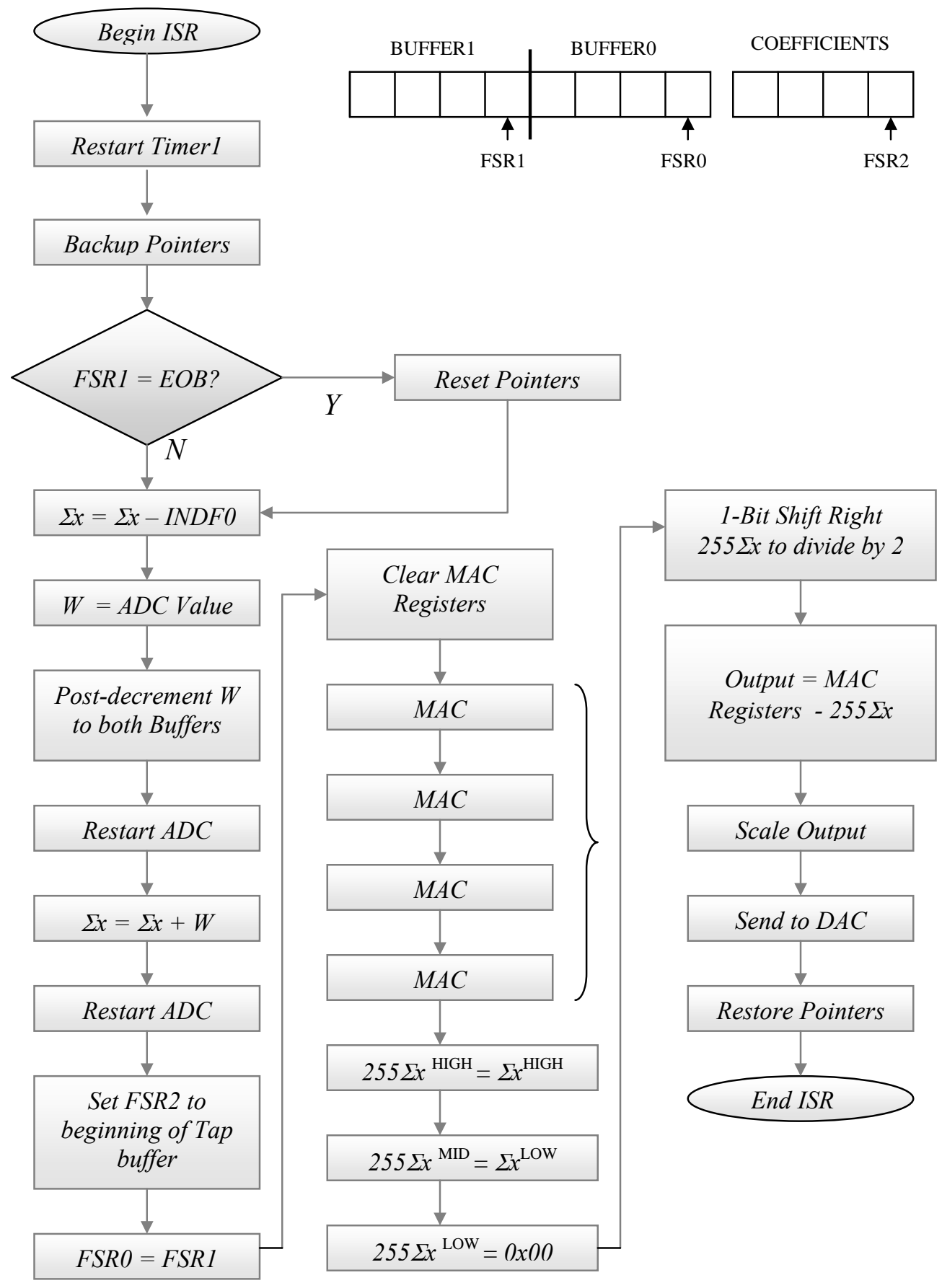

Figure 4.3.43 Fastest Execution Implementation for PIC $18 f 452$ 


\subsubsection{Implementation for Efficient RAM utilization}

The use of two circular buffers for storing ADC values is at times not acceptable due to its extensive RAM overhead. Since the FIR filter is typically used as a supplementary application, it must therefore share the available RAM with a main application. It is for this reason a less memory greedy implementation scheme is developed.

This scheme uses most of the same ideas as the previous method. The coefficients are stored in memory in the same manner as before but the MAC cycle is computed differently because since there is only one buffer and both the end-of-buffer and beginning-of-buffer needs to be checked. The details of the circular buffer are presented in Section 3.3.5. Figure 3.3.44 is a flow diagram for the initialization routine for a 4 tap FIR filter.

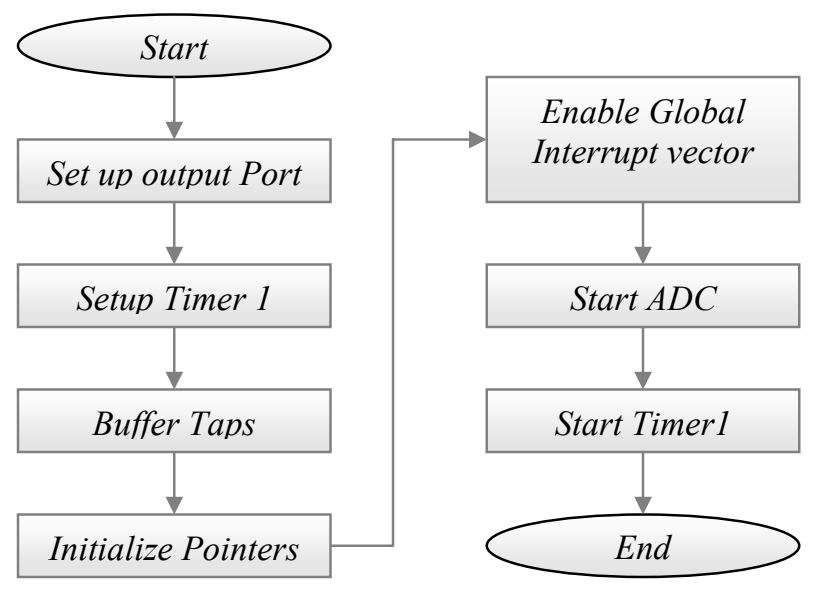

Figure 4.3.44 RAM efficient Implementation for PIC $18 f 452$ 
Figure 3.3.45 is a flow diagram for the computation routine the fastest execution time version of a 4 tap FIR filter.

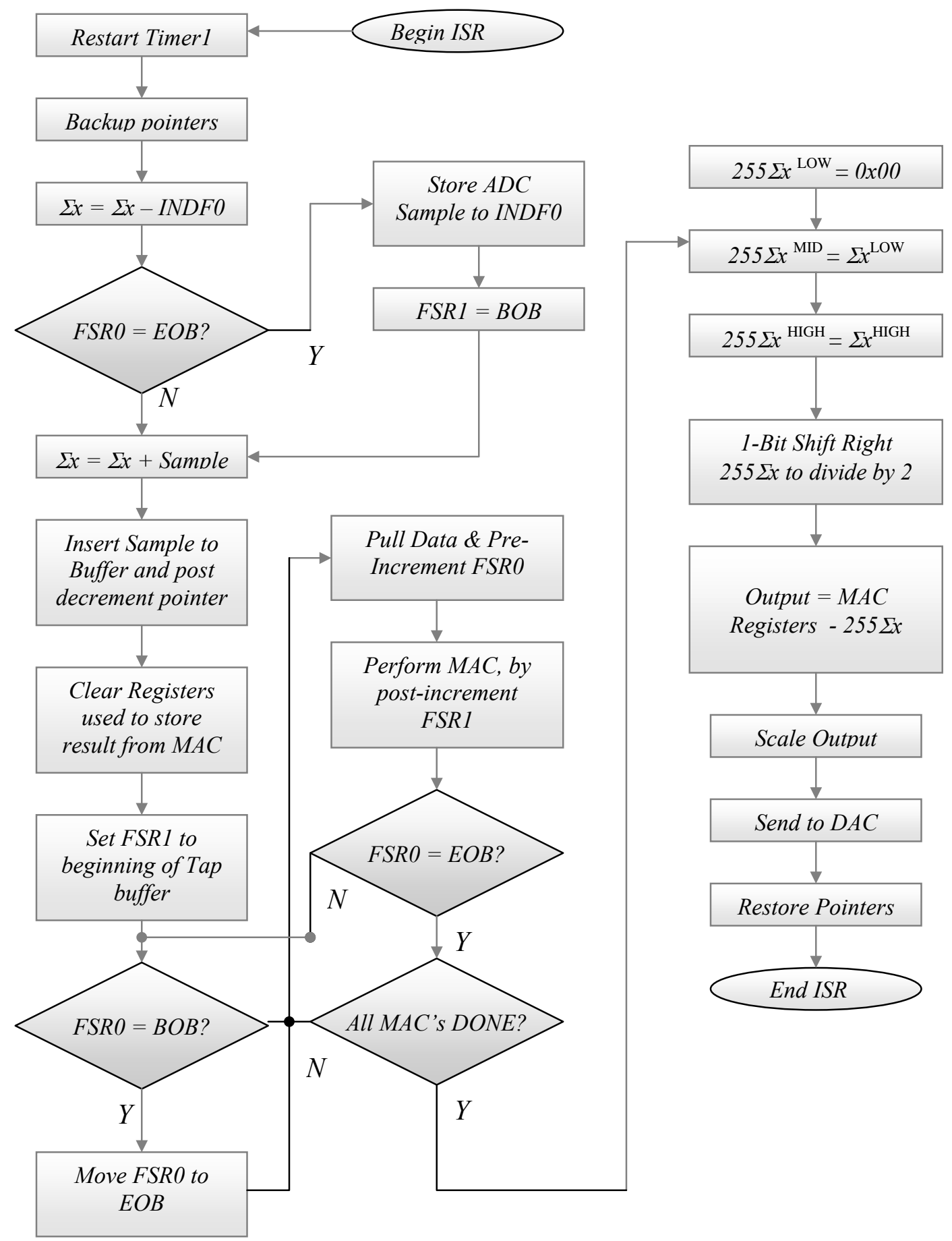

Figure 4.3.45 RAM Efficient Implementation for PIC $18 f 452$ 


\subsubsection{Implementation for Minimum Program Memory Use}

The implementation strategy is exactly like the first one where maximum execution speed was attained. In order to reduce program size, the main loop for the MAC cycle is not unrolled. Instead three more instructions are added into the MAC cycle. The computation cycle is shown in Figure 3.3.46.

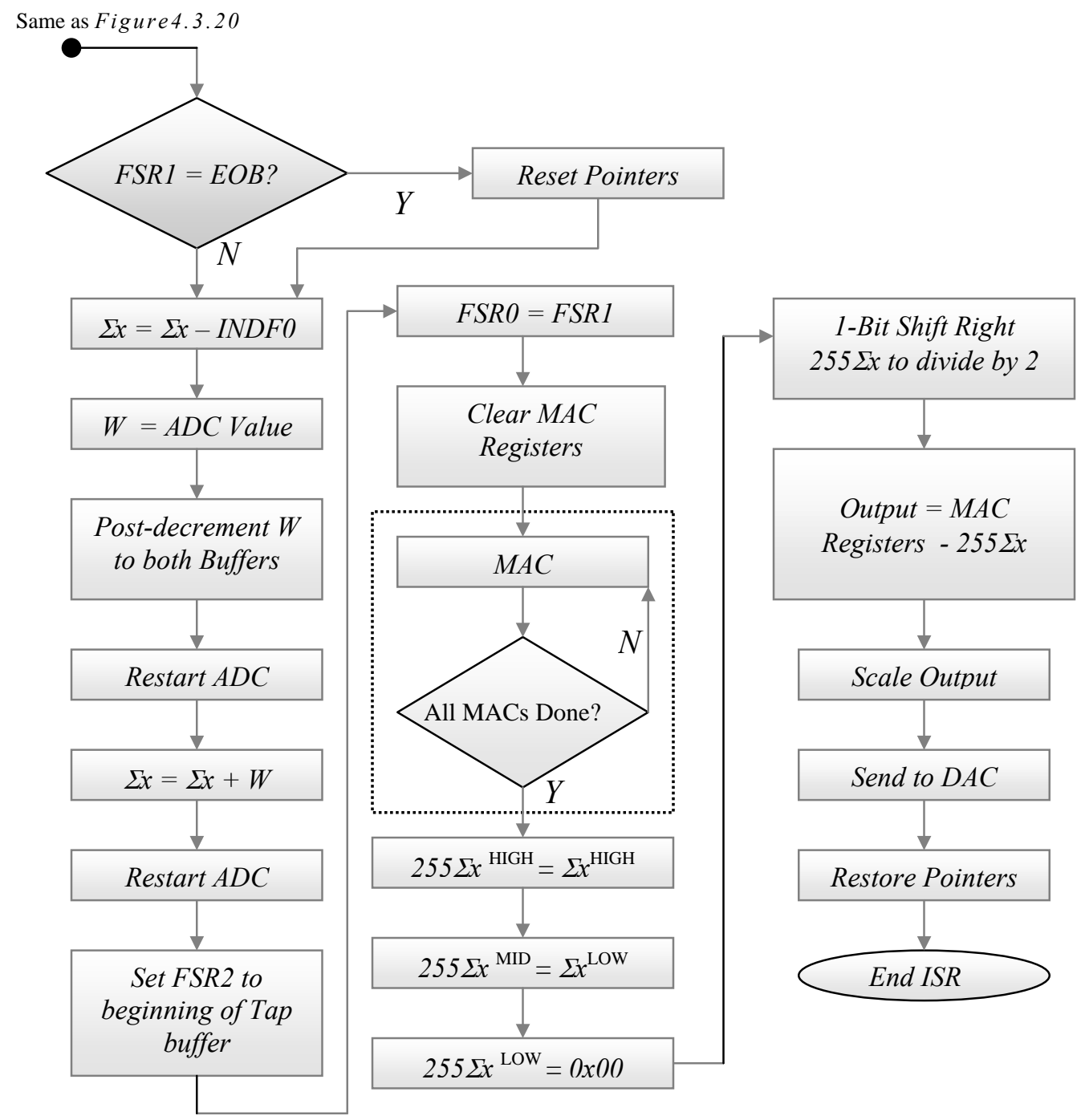

Figure 4.3.46 Minimum Program Size Implementation 


\subsection{Implementation of the floating-point LMS filter}

Unlike the FIR filters that have predetermined coefficients, implemented as constant data, the coefficients of the Least-Mean Square (LMS) filter are adaptive and continuously change as a response to input. Due to this reason, several complications must be dealt with while designing and implementing them in hardware. Since the coefficients or filter weights change with input, they may grow so large they overflow the word-space assigned to them during design time.

Stability of the LMS filter is not as easily guaranteed as it is for FIR filters. The constantly adapting coefficients are controlled by a fixed value called the learning-rate. Determining an optimal value for the learning rate requires experience gained from simulations and as the order of the adaptive filter increases, thus choice for an appropriate learning rate becomes even less intuitive. Rigorous simulations were conducted before attempting to perform real-time implementation.

The choice of the floating-point system was used to perform the implementation because the floating-point system provides both convenience and degree of immunity against both roll-off errors as well as allowing for wider latitude in the selection of learning-rate.

\subsection{The compilers floating point system}

The compilers built in math abilities were evaluated to perform the necessary filter computations but later found to be inadequate because they were extremely slow. The lack of speed is attributed to several factors. Firstly, the compiler used generic routines that are designed to work on the entire PIC family rather than applying hardware specific optimizations for the PIC18452 chip. Secondly the generic algorithms are 
optimized to be compact and not for speed. This decision is certainly well warranted as floating point algorithms written for chips that do not contain floating point hardware can use a lot of code and the lower members in the PIC family have modest sized program memories.

In order to realize the LMS filter on the PIC 18 chip it became necessary to first develop a set of floating-point routines that are optimized for the hardware at hand. New math routines were optimized for speed and designed to perform floating-point calculations much faster than the compilers generic algorithms. A standard fixed-point realization might have been more efficient however in the long run a highly optimized floating point library is far more useful as it is a reusable resource and easily applied to many other projects in the future.

\subsection{Floating-Point Word lengths}

The word lengths used to define the stored values were selected from information gathered from simulations. Figure 3.6.1 shows the word lengths that were assigned to the floating-point format numbers were used in the implementation of the LMS filter.

\begin{tabular}{||c||c||c||c||}
\multicolumn{1}{c}{ 1bit } & \multicolumn{1}{c}{ 8bits } & \multicolumn{1}{c}{ 8bits } & 8bits \\
\hline SIGN & FRACL & FRACH & REAL \\
\hline
\end{tabular}

Figure 3.6.1 Assigned Word Length for Floating Point Format

The allocated word space is 1-bit for sign, 16-Bits for the fractional part of the number and 8-bits for the real part of the number. This allowed for the possible range of [255.000000 to -255.000000$]$ with the smallest possible magnitude of 0.000015. This was determined to be sufficient resolution to be able to handle the computation requirements of the LMS filter. The next step was to develop functions that would 
conveniently perform type conversions from the standard IEEE floating point to this modified floating point. Additionally other functions were developed to perform hardware-optimized operations such as signed multiplication and signed addition and a high-speed re-scaling algorithm was added to convert a number between [ $\left.\begin{array}{ll}0 & 255\end{array}\right]$ to [ $\left.-0.50 .5\right]$.

The following ideas were used to accelerate floating-point mathematics using the PIC hardware. Parameter passing was found to be the first obvious over-head because each math operation required the passing of variables into temporary ones that were then used to compute results. The computed result needed to be passed to the output variable. It takes 2 cycles to move a single byte from one register to another and considering large numbers occupy up to 4 bytes a total of 24 cycles were spent simply in the parameter passing. This overhead is easily avoided if hardware pointers are used to directly reference data. Since The PIC chip has 3 hardware pointers, 2 are used to reference the two input parameters and the last one is used to reference the output parameter. This allows efficient movement of data through memory and since the pointers auto increment or decrement, additional cycles are not lost to pointer overhead.

Table 3.6.1: Function list developed for floating point math on PIC

\begin{tabular}{|c|c|c|}
\hline FUNCTION & DESCRIPTION & TIME@40Mhz \\
\hline void fixIeee(* float, *mfloat) & IEEE float -> modified float & Worst case (40us) \\
\hline void fix8x16(float, *mfloat) & modified float -> IEEE float & Worst case(40us) \\
\hline void add(void) & adds 2 modified signed floats & 5 us \\
\hline void mul(void) & multiply 2 modified signed floats & 3 us \\
\hline $255 /$ integer $->$ mfloat & Normalize [0 255] -> [0.00 0.99] & $400 \mathrm{~ns}$ \\
\hline
\end{tabular}




\subsubsection{Algorithm developed for floating point multiplication}

The multiplication algorithm operates on two floating-point variables each stored in RAM in the format described Figure 3.6.2.

Number $A$

\begin{tabular}{|c|c|c|c|c|c|c|c|c|c|c|}
\hline & Sign & FracL & FracH & Real & & Sign & FracL & FracH & Real & \\
\hline 0x37 & $\begin{array}{c}0 \times 38 \\
\mathbf{0}\end{array}$ & $\begin{array}{c}0 \times 39 \\
\mathbf{0}\end{array}$ & $\begin{array}{c}0 \times 40 \\
\mathbf{0}\end{array}$ & $\begin{array}{c}0 \times 41 \\
\mathbf{0}\end{array}$ & $0 \times 42$ & $\begin{array}{c}0 \times 43 \\
\mathbf{0}\end{array}$ & $\begin{array}{c}0 \times 44 \\
\mathbf{0}\end{array}$ & $\begin{array}{c}\text { 0x45 } \\
\mathbf{0}\end{array}$ & $\begin{array}{c}0 \times 46 \\
\mathbf{0}\end{array}$ & $0 \times 47$ \\
\hline
\end{tabular}

Number C (Result)

\begin{tabular}{|c|c|c|c|c|c|}
\hline & Sign & FracL & FracH & Real & \\
\hline $0 \times 60$ & $\begin{array}{c}\text { 0x61 } \\
\mathbf{0}\end{array}$ & $\begin{array}{c}\text { 0x62 } \\
\mathbf{0}\end{array}$ & $\begin{array}{c}\text { 0x63 } \\
\mathbf{0}\end{array}$ & $\begin{array}{c}\text { 0x64 } \\
\mathbf{0}\end{array}$ & $0 \times 65$ \\
\hline
\end{tabular}

Figure 3.6.2 Memory footprint of floating point numbers

Numbers A, B and C are stored in RAM and each is given 4 bytes of memory. FracL and FracH make up the fractional portion given 16-bits of storage the real part or the integer part is given 8-Bits of storage each. In order to save time from parameter passing, pointers FSRO, FSR1 and FSR2 are used to manipulate the floating-point variables. The multiplication algorithm is designed to take advantage of both the hardware-multiplier to compute the products of integer and fractional portions and PIC18 memory addressing features that provide single cycle pointer operation and advance. 
The formal algorithm for multiplication is described next. The three numbers are setup in memory as shows in Figure 3.6.2.

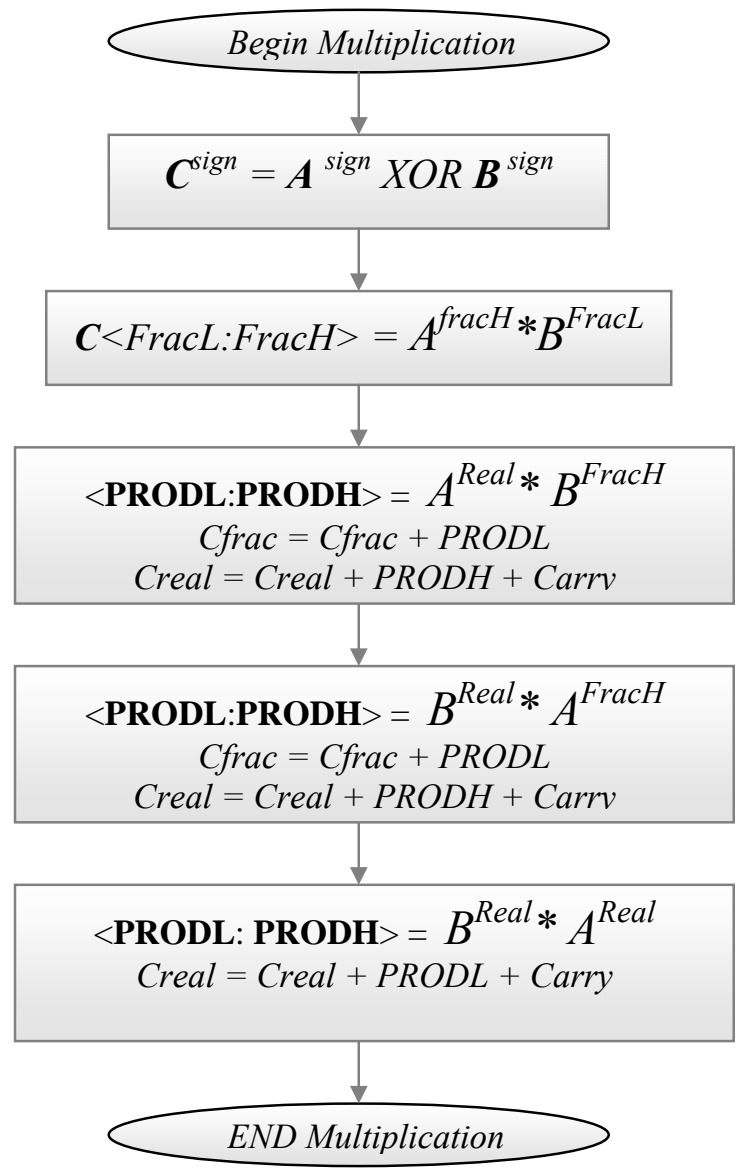

Figure 3.6.3 Developed Algorithm for Multiplication

The step-by-step illustration of the above algorithm is presented next along with the assembly code that was written to implement it. The purpose of such a detailed presentation is to clarify pointer use in the PIC18F452 chip and to show the functionality of the algorithm.

Step 1 shown in Figure 3.6.4, the numbers to be multiplied are stored in $R A M$ and each is given it's own dedicated pointer as shown below. The first step is to determine the sign of the computed product. 
Evaluating the $X O R$ of the signs of the two numbers being multiplied results in the sign of $C$.

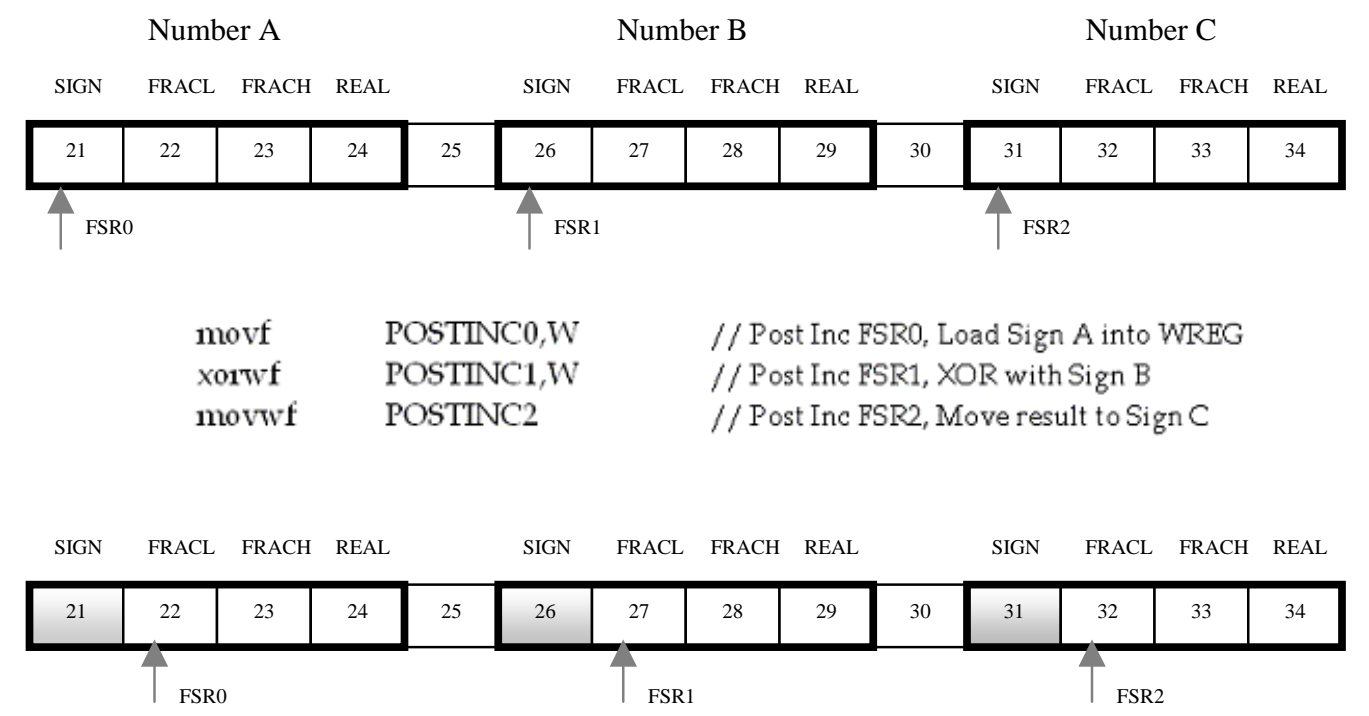

Figure 3.6.4 Multiplication Step 1: $\mathbf{C}^{\text {sign }}=\mathbf{A}^{\text {sign }}$ XOR $\mathbf{B}^{\text {sign }}$

Step 2 shown in Figure 3.6.5, the fractional portion of the result $C$ is evaluated next by computing the product: $\mathbf{C}<$ FracL: FracH $>=$ $A^{\text {fracH}} * B^{\text {fracH }}$.

$\begin{array}{lll}\text { movf } & \text { PREINC0,W } & \text { // Pre Inc FSRO, Move AFracH to WREG } \\ \text { mulwf } & \text { PREINC1 } & \text { // Pre Inc FSR1, Multiply WREG with BFracH } \\ \text { movff } & \text { PRODL, POSTINC2 } & \text { // Post Inc FSR2, Move Product Low to CfracL } \\ \text { movff } & \text { PRODH, INDF2 } & \text { // Move Product High to CfracH }\end{array}$

\begin{tabular}{c|c|c|c|c|c|c|c|c|c|c|c|c|c|} 
SIGN & FRACL & FRACH & REAL & & SIGN & FRACL & FRACH & REAL & \multicolumn{2}{c|}{ SIGN } & FRACL & FRACH & REAL \\
\hline 21 & 22 & 23 & 24 & 25 & 26 & 27 & 28 & 29 & 30 & 31 & 32 & 33 & 34 \\
\hline
\end{tabular}

Figure 3.6.5 Multiplication Step 2: $\mathbf{C}<$ FracL: FracH $>=A^{\text {fracH}} * B^{\text {fracH }}$ 
Step 3 shown in Figure 3.6.6, The real part of result $C$ is evaluated next by computing the product of $A^{\text {Real } *} B^{\text {FracH }}$ and adding the low-byte of the product to Cfrac and adding the high-byte of the product to Creal with the carry from the previous addition.

\begin{tabular}{|c|c|c|c|c|c|c|c|c|c|c|c|c|c|}
\hline \multirow[b]{2}{*}{ SIGN } & $\begin{array}{l}\text { hovf } \\
\text { hudwf } \\
\text { novff } \\
\text { ddwef } \\
\text { novff } \\
\text { ddwfe }\end{array}$ & & $\begin{array}{l}\text { PREI } \\
\text { POST } \\
\text { PROD } \\
\text { POST } \\
\text { PROD } \\
\text { POST }\end{array}$ & $\begin{array}{l}\mathrm{CO}, \\
\mathrm{NC} \\
\mathrm{L}, \mathrm{W} \\
\mathrm{NC} \\
\mathrm{H}, \mathrm{W} \\
\mathrm{EC}\end{array}$ & $\begin{array}{l}\mathrm{EG} \\
\mathrm{EG} \\
\mathrm{F}\end{array}$ & & $\begin{array}{l}/ / \text { PreI } \\
/ / \text { Post } \\
/ / \text { Mov } \\
/ / \text { Post } \\
/ / \text { Mov } \\
/ / \text { Post }\end{array}$ & $\begin{array}{l}\text { nc FS } \\
\text { Inc F } \\
\text { e Pro } \\
\text { Inc F } \\
\text { e Pro } \\
\text { Dec I }\end{array}$ & $\begin{array}{l}0, \mathrm{~N} \\
21, \mathrm{I} \\
1 \mathrm{ct} \\
22, \\
\mathrm{1ct} 1 \\
\mathrm{R} 2,\end{array}$ & $\begin{array}{l}\text { fe } \mathrm{AF} \\
1 \text { tipl } \\
w \text { to } \\
\text { d wit } \\
\text { h to } \\
\text { d (W }\end{array}$ & $\begin{array}{l}\mathrm{CH} \text { to } \\
\text { WREG } \\
\text { REG } \\
\text { W sto } \\
\text { REG } \\
\text { Carry }\end{array}$ & $\begin{array}{l}\text { re in } \mathrm{C} \\
+\mathrm{CfracL}\end{array}$ & freal \\
\hline & FRACL & FRACH & REAL & & SIGN & FRACL & FRACH & REAL & & SIGN & FRACL & FRACH & REAL \\
\hline 21 & 22 & 23 & 24 & 25 & 26 & 27 & 28 & 29 & 30 & 31 & 32 & 33 & 34 \\
\hline
\end{tabular}

Figure 3.6.6 Multiplication Step 3: $A^{\text {Real* }} B^{\text {FracH }}$

Step 4 shown in Figure 3.6.7, The real part of result $C$ is evaluated next by computing the product of $A^{\text {FracH }} * B^{\text {Real }}$ and adding the low-byte of the product to Cfrac and adding the high-byte of the product to Creal with the carry from the previous addition.

$\begin{array}{lll}\text { decf } & \text { FSR0L,F } & \text { // Dec FSRO, Point to AReaL } \\ \text { movf } & \text { POSTINC0,W } & \text { // Post Inc FSR0, Move AReaL To WREG } \\ \text { mulwf } & \text { INDF1 } & \text { // Multiply BfracH with WREG } \\ \text { movff } & \text { PRODL,WREG } & \text { // Move Product Low to WREG } \\ \text { addwf } & \text { POSTINC2,F } & / / \text { Post Inc FSR2, add WREG to CReaL } \\ \text { movff } & \text { PRODH,WREG } & \text { // Move Product High to WREG } \\ \text { addwfic } & \text { INDF2,F } & / / \text { Add (carry+WREG+CfracH)->CFeacH }\end{array}$

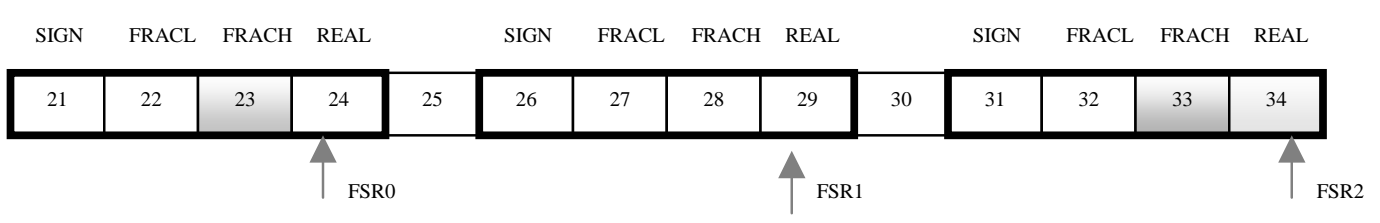

Figure 3.6.7 Multiplication Step 4: $\mathrm{A}^{\text {FracH* } *} \mathrm{~B}^{\text {Real }}$ 
Step 5 shown in Figure 3.6.8, The real part of number $A$ and the real part of number $B$ are multiplied next and the low byte of the result is added to the real part of number $C$. This concludes the multiplication operation.

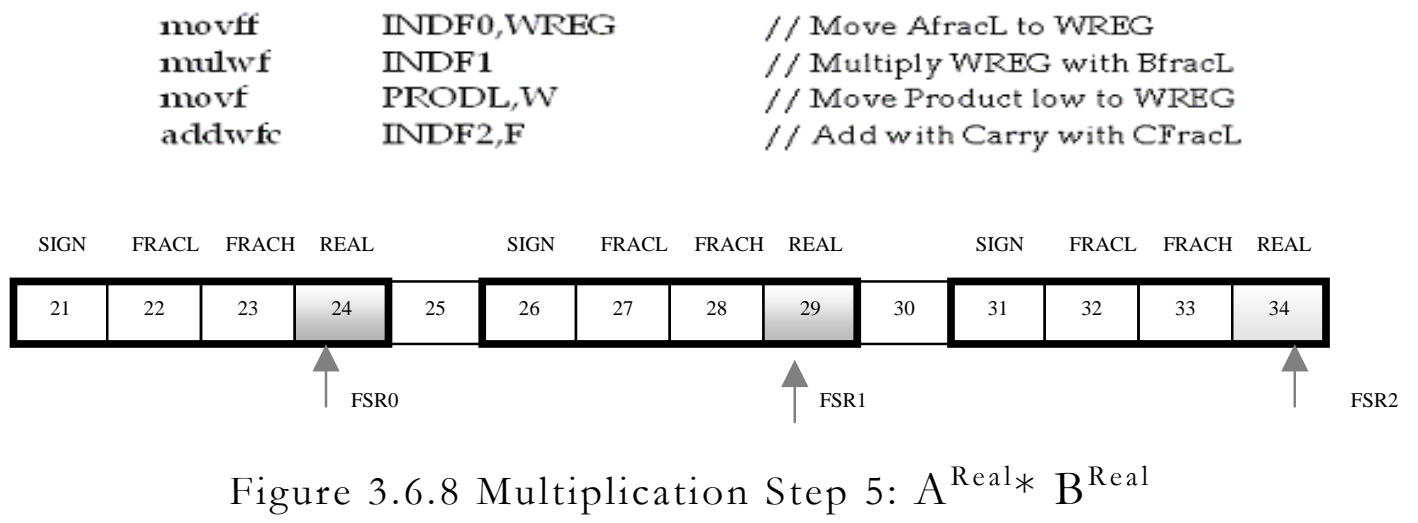

There are no conditions to be checked in the algorithm hence the two-stage pipeline of the PIC chip is constantly maintained. All instructions are single cycle $(100 \mathrm{~ns})$ with the exception of the register-toregister move instruction (movff), which is two-cycle (200ns). The total time used by this algorithm is 3 us. An additional advantage is that this algorithm always takes the same amount of time to execute. The compilers worst case floating point multiplication algorithm is $45 \mathrm{us}$ according to their published manual [3].

The use of the multiplication algorithm in C-language is demonstrated next. No condition checking is available to determine and warn users about over and underflows in the interest of efficiency. Figure 3.6.9 is the $\mathrm{C}$-code needed to use the multiply function.

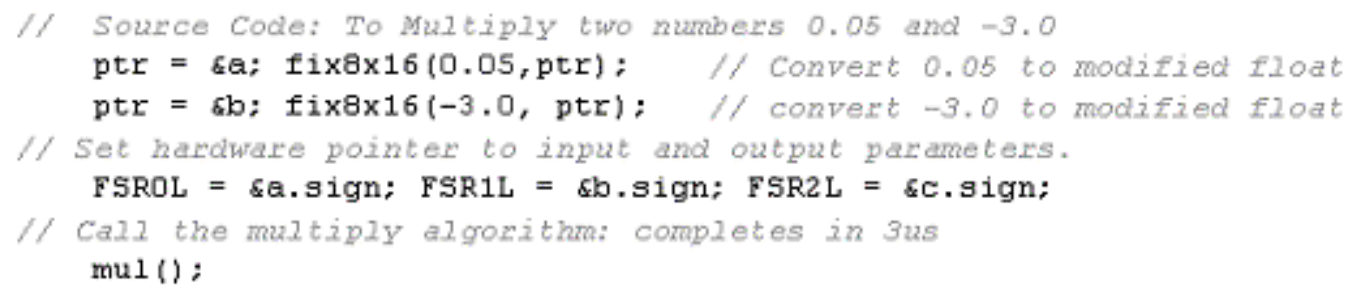

Figure 3.6.9 C-Code for floating point multiplication 


\subsubsection{Calling The Floating-Point Add in C}

The algorithm developed for performing floating-point addition was implemented along the same lines as the multiplication algorithm. Both operate on the same type of data format and both use hardware pointers to reference data.

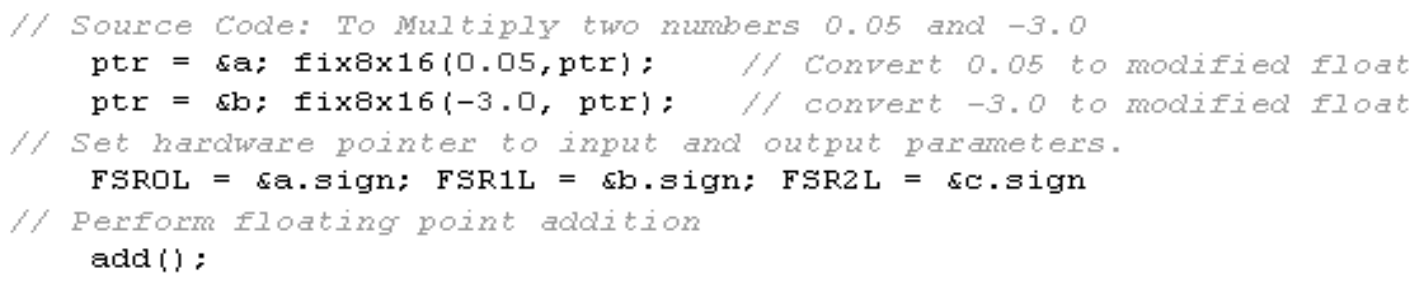

Figure 3.6.10 C-Code for floating point addition

\subsubsection{Algorithm developed for floating-point addition}

The floating-point addition algorithm was developed keeping in mind the fact that nether of the input parameters are corrupted during the addition process. To clarify the point assumes that two numbers $A$ and $B$ are being added to calculate C. After the addition is completed neither A or B will change in value. The algorithm would have been slightly shorter if this constraint were removed, however we would loose the ability to perform MAC operations where parameters are added to themselves. The detailed algorithm is illustrated in Figure 3.6.11. 

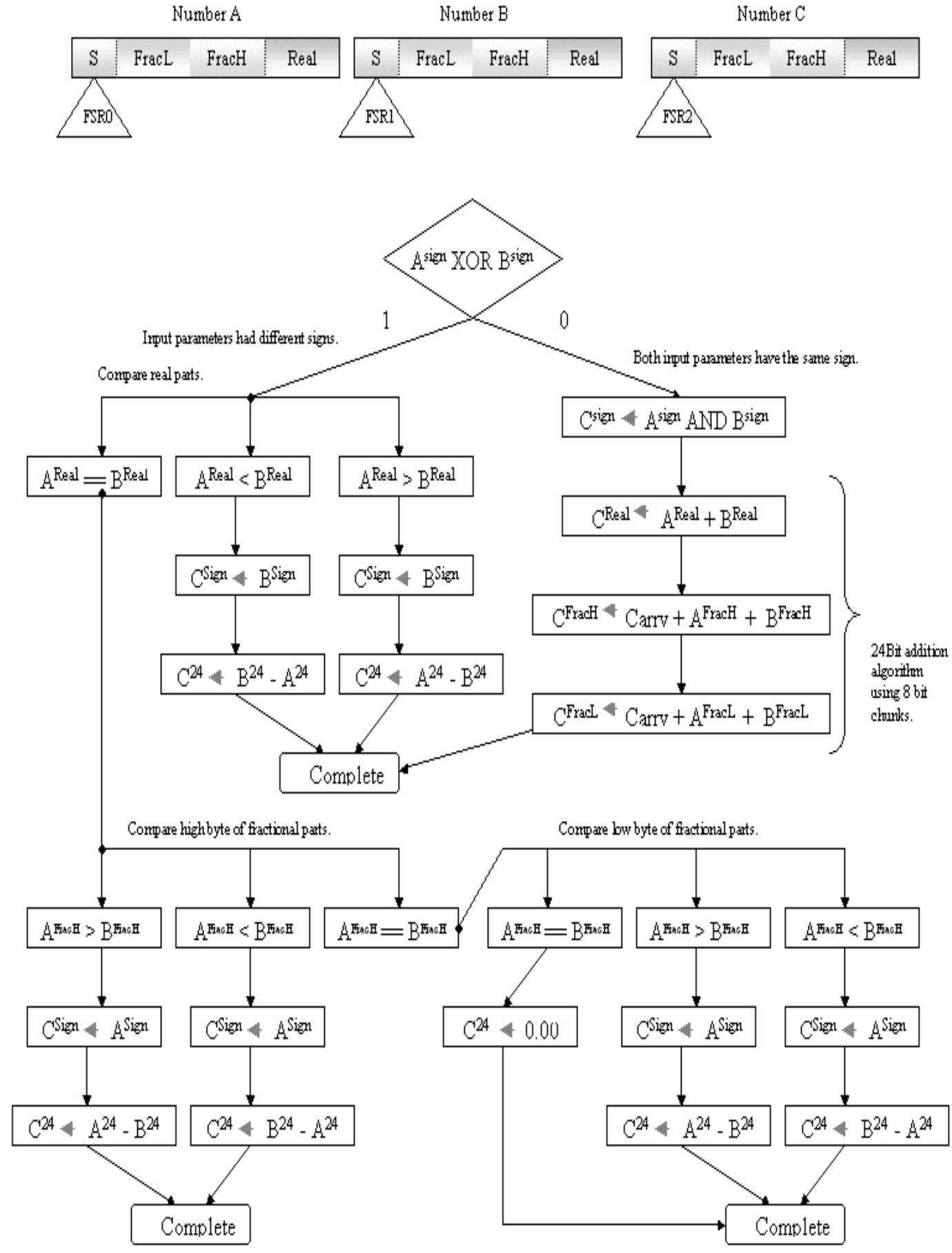

Figure 3.6.11 Developed Algorithm for Addition 


\subsubsection{Converting integer to floating-point format}

A quick way to convert integer values to floating-point needs to be implemented because data read in by the analog-to-digital ranging from [0 255] needs to be converted to float range between $[-0.5,0.5]$.

$$
\text { Nfloat }=(\text { float }) \frac{(\text { unsigned } \text { int }) N-128}{256}
$$

A division operation is out of the question because it's computationally prohibitive if performed in real time. Installing a look up table was the first option however a more elegant approximation is given in Figure 3.6.12.

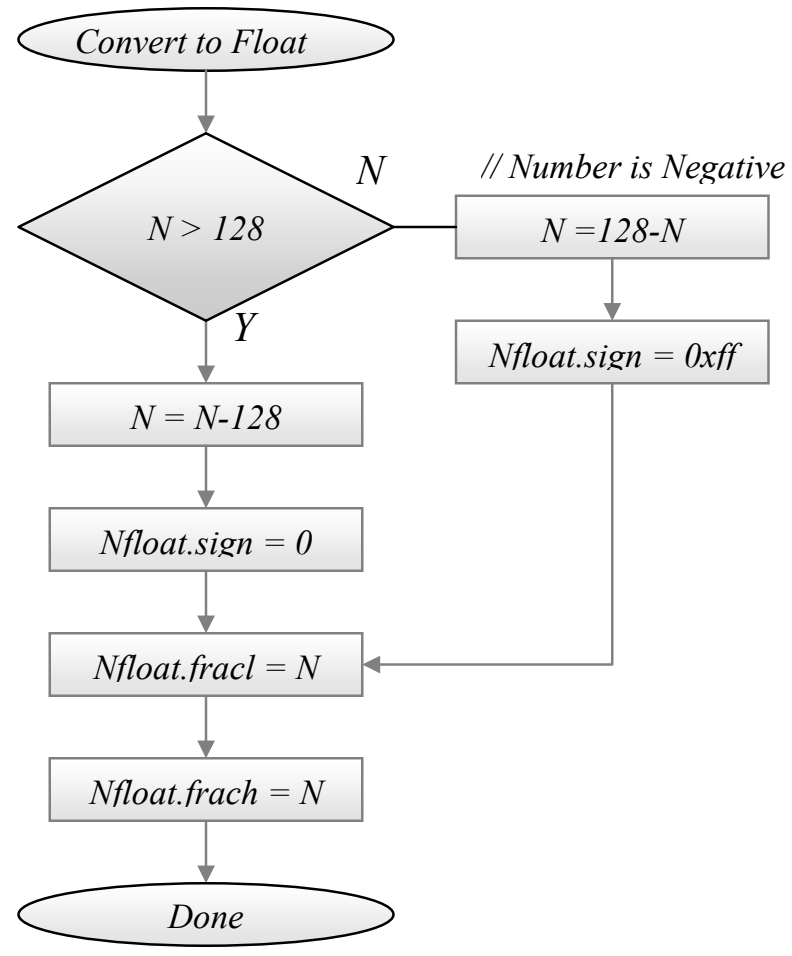

Figure 3.6.12 Developed Algorithm for Multiplication 


\subsection{Implementation of a $4^{\text {th }}$ order real-time LMS algorithm}

The topology of the LMS algorithm used is illustrated first. The filter samples two channels where $\mathrm{Y}_{\mathrm{k}}$ is the signal that needs to be filtered and $\mathrm{X}_{\mathrm{k}}$ is the reference. $\mathrm{W}_{0}, \mathrm{~W}_{1}, \mathrm{~W}_{2}$ and $\mathrm{W}_{3}$ are all weights or filter coefficients of the LMS filter. These are initialized as 0 however as the filter trains the weights converges to a solution value. The variable $e_{k}$ is called the error signal and it is both the output of the filter and the feedback signal that trains the filter weights.

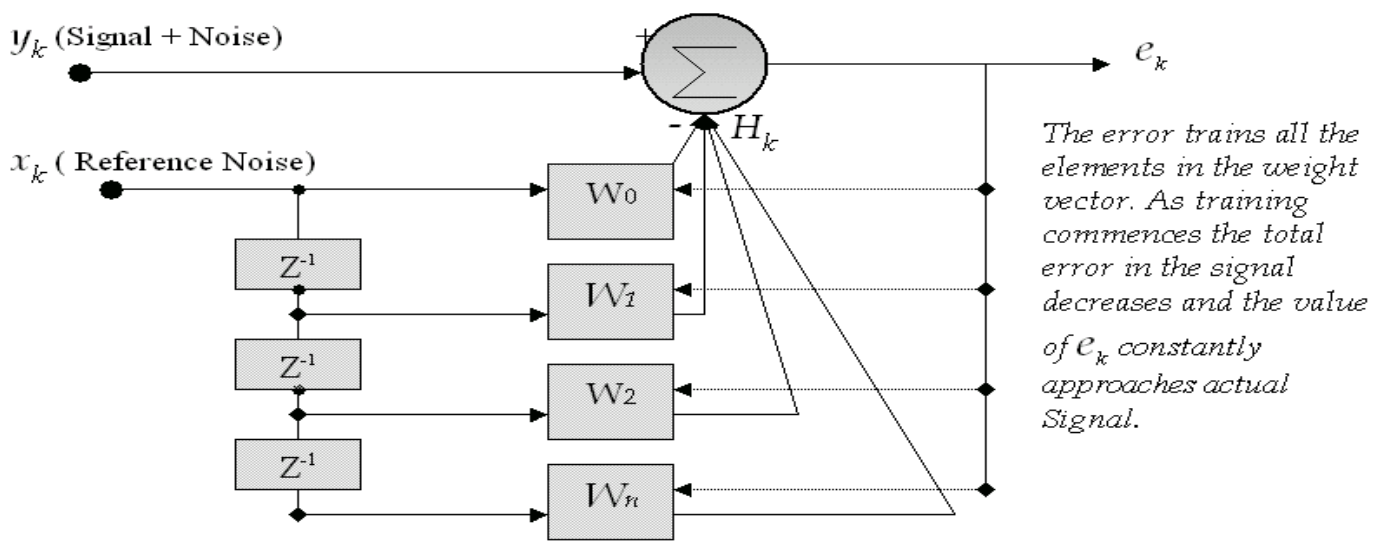

Figure 3.7.1 Fourth Order LMS Filter

The filter equations are to be implemented and computed in real-time in the PIC 18F452 chip are presented next. The error signal $e_{k}$ is evaluated by a dot product of the weight vector $W_{k}$ and the reference signal vector $\mathrm{X}_{\mathrm{k}}$ and is calculated using the Equation 3.13.

$$
e_{k}=y_{k}-\left[\begin{array}{l}
W_{0} \\
W_{1} \\
W_{2} \\
W_{3}
\end{array}\right] *\left[X_{0}^{\text {newest }}, X_{1}, X_{2}, X_{n}^{\text {oldest }}\right]
$$


After each iteration the filter weights or sometimes known as filter coefficients must be updated using the feedback error value $e_{k}$ and update values for each of the weights are calculated separately using the following Equations 3.15a,b,c and d.

$$
\begin{aligned}
& W_{1}{ }^{\prime}=W_{1}+\varepsilon^{*} e_{k} * X_{1} \\
& W_{2}{ }^{\prime}=W_{2}+\varepsilon^{*} e_{k} * X_{2} \\
& W_{3}{ }^{\prime}=W_{3}+\varepsilon^{*} e_{k} * X_{2} \\
& W_{n}{ }^{\prime}=W_{n}+\varepsilon^{*} e_{k} * X_{n}
\end{aligned}
$$

Where,

$\mathrm{e}_{\mathrm{k}}$ : Filter Output (used to train the filter weights)

Wn: Weight Vector also known as Filter Taps

$\varepsilon$ : Learning Rate (controls rate of descent)

n: Filter Order

All variables used in here are in the floating-point format and the floating-point math algorithms described in the previous section are used to handle the computational load of the filter.

\subsubsection{Sampling noise and reference for LMS filter}

Two channels of the PIC18F452 ADC are used to sample for the LMS filter. The first channel samples the noise $\mathrm{Yk}$ and the second channel samples the reference Xk. Shown in Figure 3.7.2.

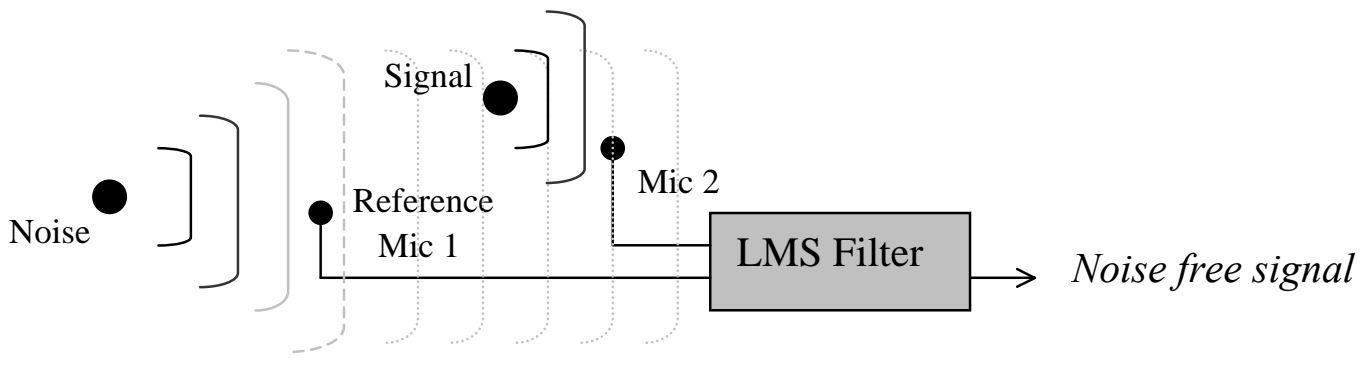

Figure 3.7.2 Sampling for LMS 
The LMS filter doesn't have to necessarily be used with audio as the illustration above suggests. It can be used with any two signals that are correlated. The Analog to digital converters are used with 8-bits of precision and are configured exactly like the FIR filters except 2 channels are used for this filter instead of one. The sampling used to implement a $4^{\text {th }}$ order LMS filter was $8000 \mathrm{~Hz}$.

Configuring the ADC involved writing the appropriate registers as shown in Figure 3.7.3.

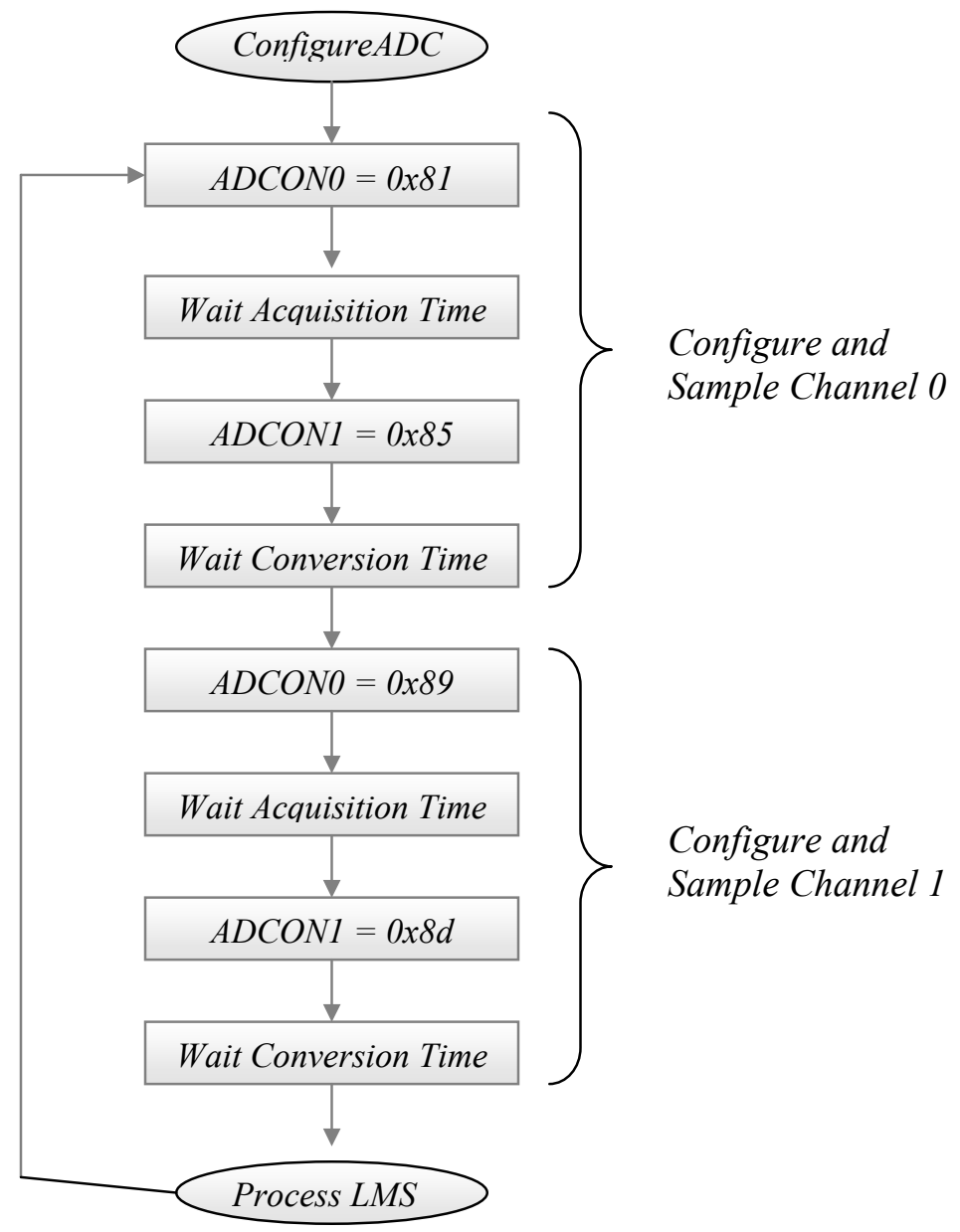

Figure 3.7.3 Configuring ADC for Sampling Two Channels 


\subsubsection{Program Outline for $4^{\text {th }}$ order LMS filter}

Like the FIR filter, the Implementation scheme for the LMS filter is also presented as two routines. Firstly the initialization routine, where the variables and buffers are initialized and all the hardware that plays a part in LMS are initialized for use and secondly the computation routine in which the LMS algorithm is computed. The computation routine, like the FIR filter, runs entirely in the interrupt service routine of a timer, in this case timer 2 was used.

The floating-point variables for $\mathrm{W}_{\mathrm{k}}, \Delta \mathrm{W}_{\mathrm{k}}, \mathrm{Y}_{\mathrm{k}}, \mathrm{X}_{\mathrm{k}}, \mathrm{H}_{\mathrm{k}}, \mathrm{e}_{\mathrm{k}}$ are all declared as structures with four members, Sign, FracH, FracL, and Real. Data collected in real-time by the ADC's include one 8-bit sample value for the signal and four buffered 8-bit sample values for reference. Both signal and reference need to be converted into floating-point format before they can be processed. A single 4-point circular buffer is used to store four values of reference. Figure 3.7 .4 presents the initialization routine for the $4^{\text {th }}$ order LMS algorithm.

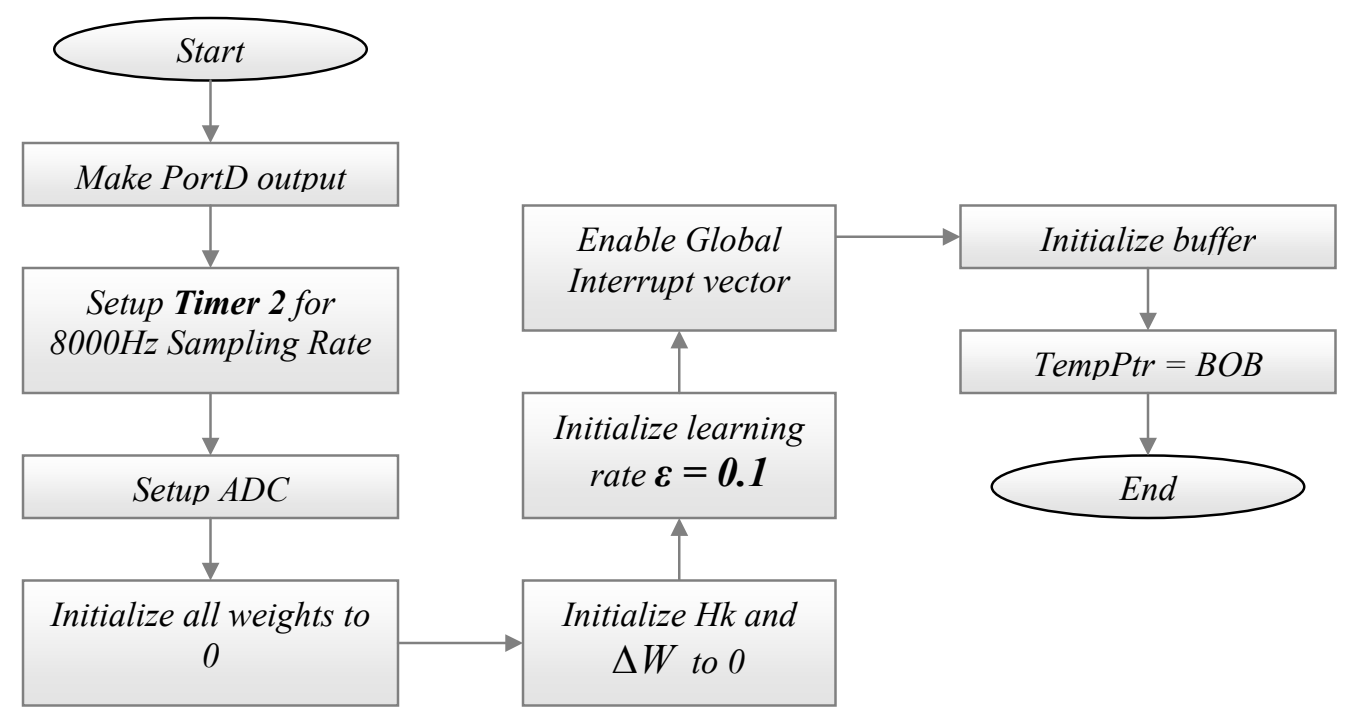

Figure 3.7.4 Initialization Routine for LMS 


\section{Computation Routine for LMS: Buffering schemes}

Most variables used in this filter are declared as global structs and are not dynamically written or read. However, the reference signal sampled by the ADC needs to be saved in chronological order for LMS calculations. A four element circular buffer was used to store and maintain the ADC samples of the reference signal as 8-bit unsigned bytes per sample as shown in Figure 3.7.5.

\begin{tabular}{|c|c|c|c|c|c|}
\hline \multicolumn{6}{|c|}{ Reference Sample Buffer } \\
\hline \multicolumn{3}{|c|}{ ВОВ } & \multicolumn{3}{|c|}{ EOB } \\
\hline 0x60 & 0x61 & $0 \times 62$ & 0x63 & 0x64 & $0 \times 65$ \\
\hline & 0 & 0 & 0 & 0 & \\
\hline
\end{tabular}

Figure 3.7.5 Four element ADC sample bvffer for LMS

The ADC samples have to be converted into floating-point format and stored in the appropriate structs efficiently before the can be used for LMS. To achieve this the structs are declared in chronological order for storing reference samples in floating-point format and placed sequentially in RAM and a single pointer is used to load all the structs with data pulled from the circular sample buffer. Once all structs are loaded they can be addressed as ordinary variables during computation time. Essentially the structs are loaded dynamically and read statically as if the were the union of four structs and a 16 byte array as shown in Figure 3.7.6. 


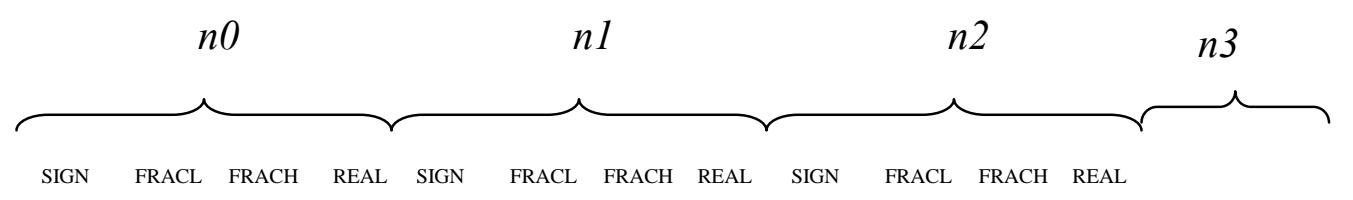

\begin{tabular}{|l|l|l|l|l|l|l|l|l|l|l|l|}
\hline 21 & 22 & 23 & 24 & 25 & 26 & 27 & 30 & 31 & 32 & 33 & 34 \\
\hline
\end{tabular} FSRO 000

Figure 3.7.6 Arangements of Structs in Memory

\section{Computation Routine for LMS: Level 1}

The Implementation of LMS algorithm in the PIC18F452 chip follows the following basic steps as shown in Figure 3.7.5. The level 1 flow diagram shows an over view of the installation. The entire algorithm is timing sensitive and there fore it runs in the ISR of timer 2.

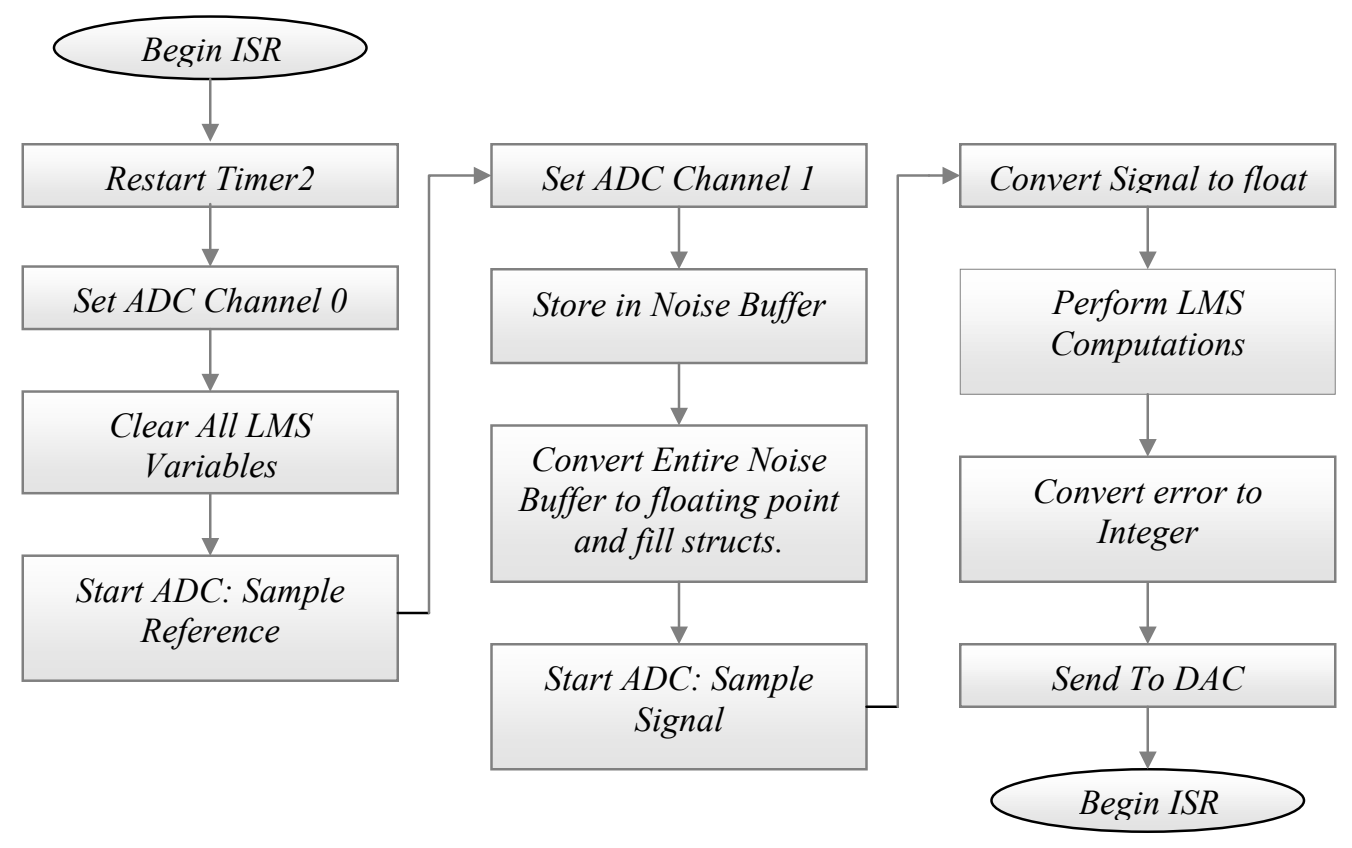

Figure 3.7.7 Level 1 Flow Diagram for LMS 
Figures 3.7.8 show the contents of the ISR in detail and Figure 3.7.9 shows in expanded form the detailed computational section of the LMS algorithm.

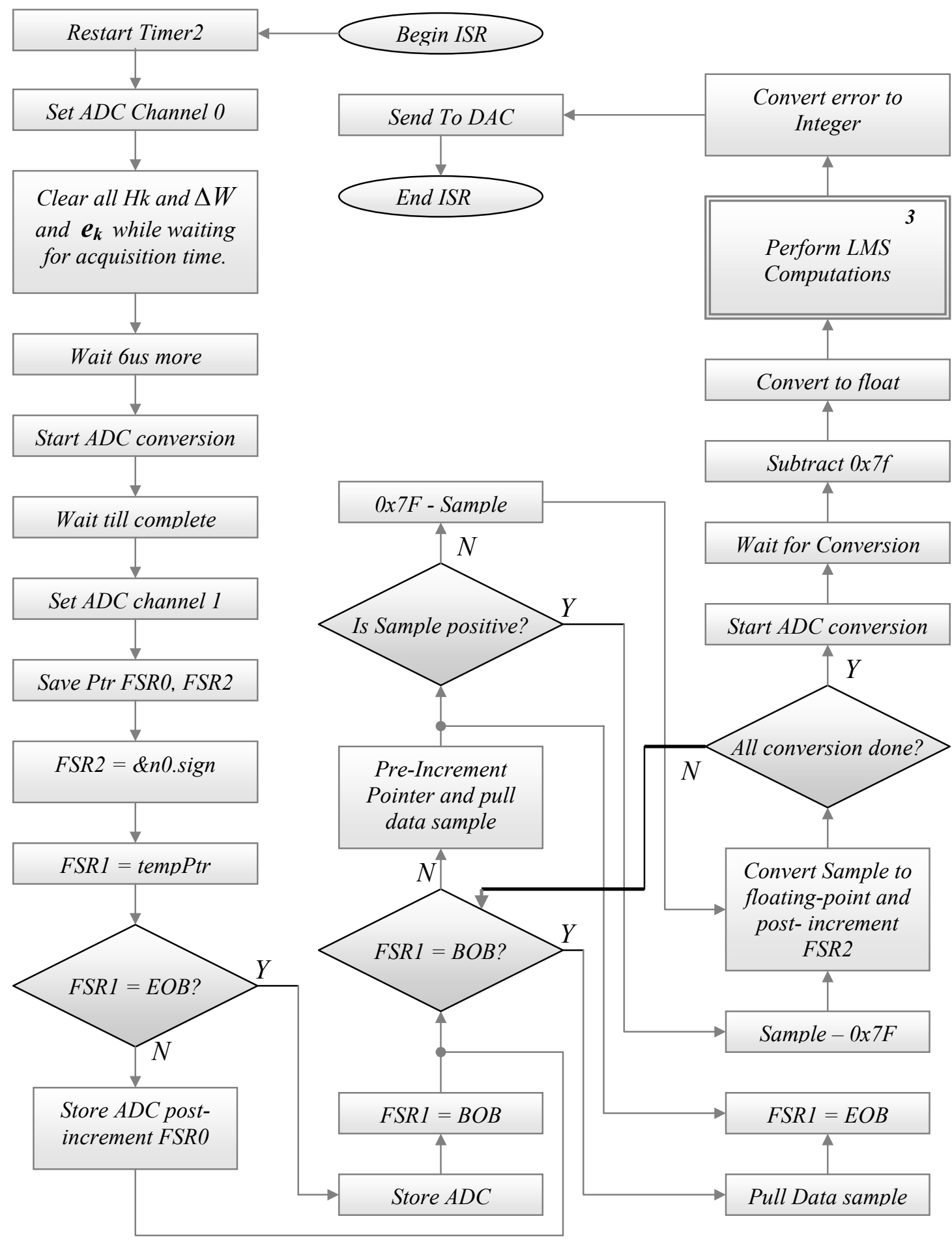

Figure 3.7.8 Level 2 Flow Diagram for LMS 
The detailed computational flow diagram of the LMS algorithm is given in Figure 3.7.9.

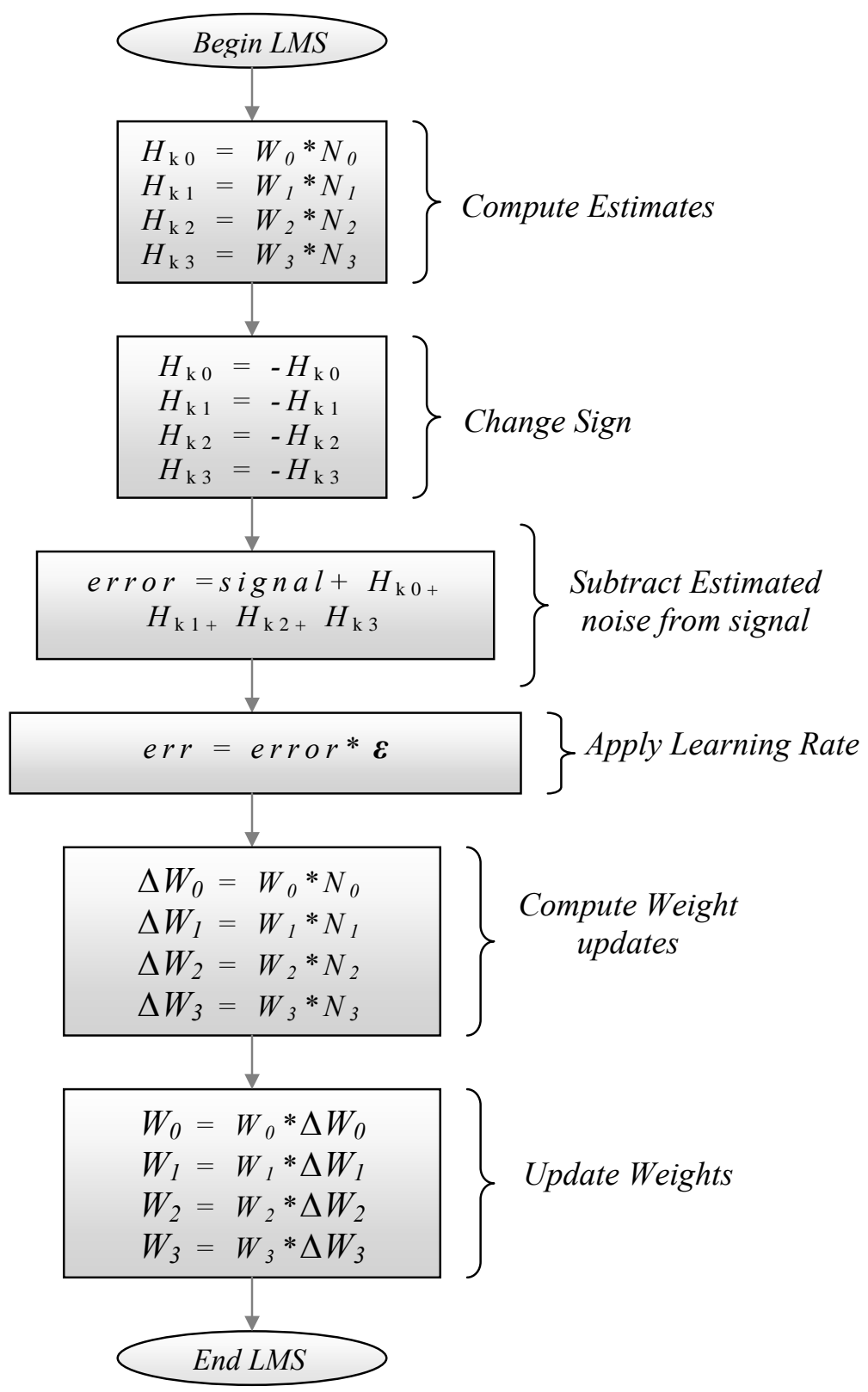

Figure 3.7.9 Level 3 Flow Diagram for LMS 


\subsection{Hardware Test Circuit}

The same test circuit was used for both FIR and LMS filter. Figure 3.8.1 is a block diagram of the circuits that are used.

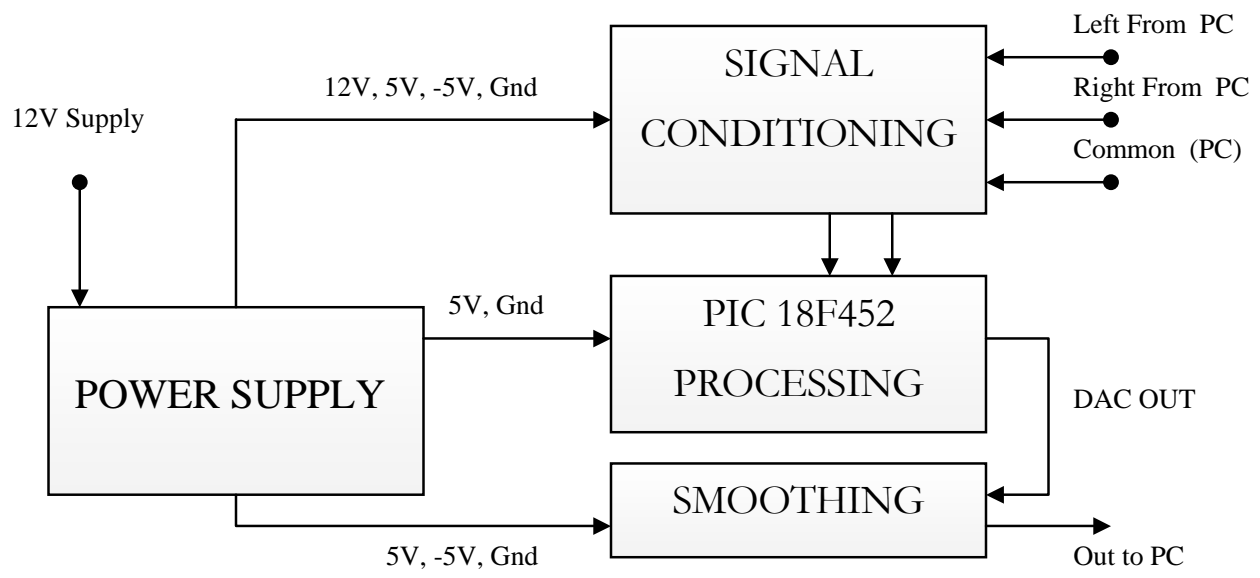

Figure 3.8.1 Block Overview of Circuit

The power supply board was developed to provide the following voltages from a unregulated $12 \mathrm{~V}+$ power supply. This board is labeled optional because it was developed purely to make convenient voltage supplies and references. The board provides the following voltages.

a. Regulated 5V / 1A (Power for PIC 18F452 and other IC's)

b. Regulated 2.5V (Offset Voltage for Amplifiers)

c. $-5 \mathrm{~V} / 100 \mathrm{~mA}$ Unregulated (DC/DC) 


\subsection{Detailed Schematic of the Power Supply}

The power supply board uses the TC1121 DC/DC converter to generate the -5 supply. The $5 \mathrm{~V}$ supply is regulated by the 7805 and the 2.5 Volt reference level is generated with a zener. All the voltages are derived from a $12 \mathrm{~V}$ external supply.

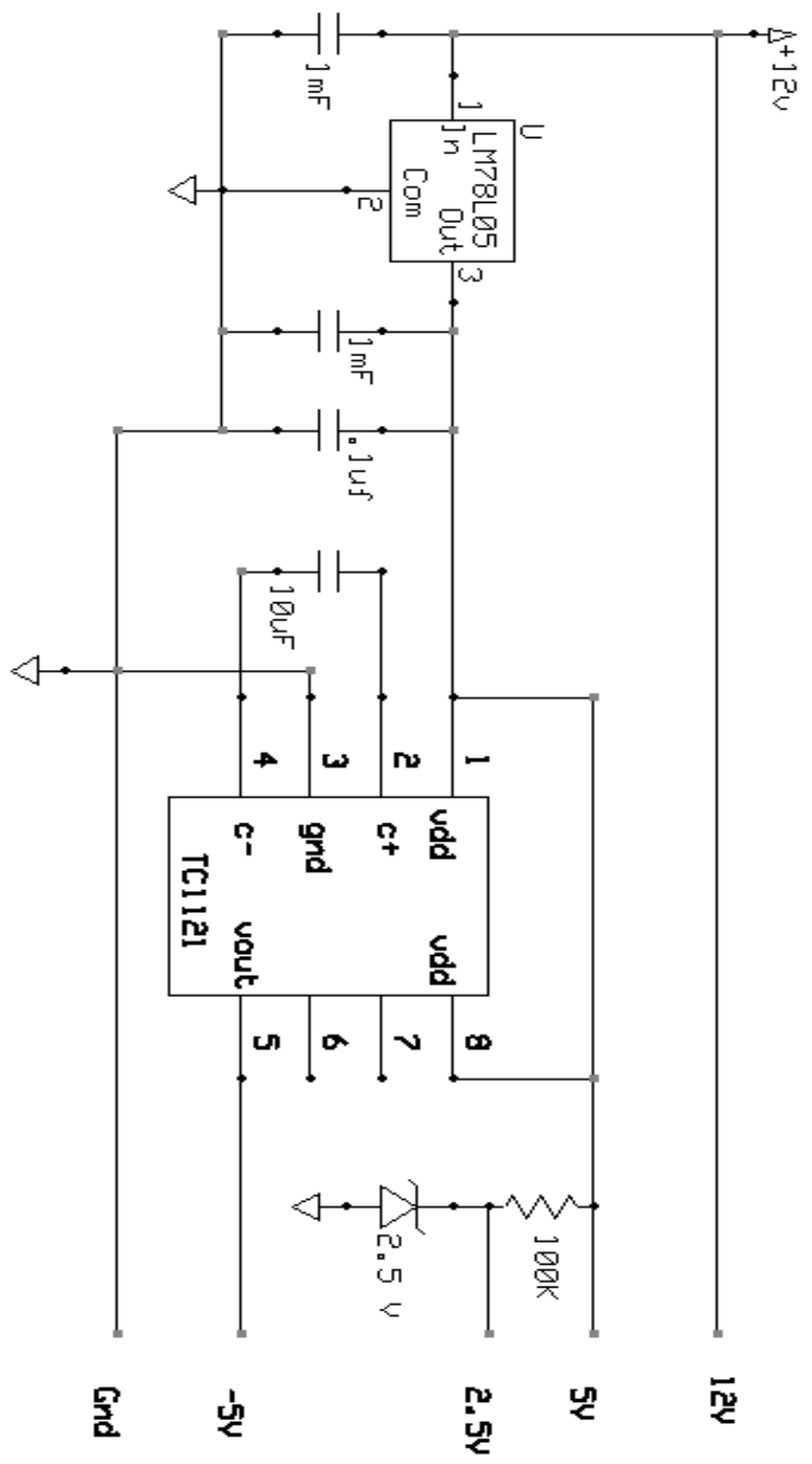

Figure 3.8.2 Power Supply Board 


\subsection{Detailed Schematic: Smoothing Filter}

The output signal from the R-2R filter must be buffered and smoothed before it can be sent for data analysis. To that end the Maxim 291 switched capacitor filter is used. The filter is clocked with a small PIC chip PIC 12F629. The firmware for the switch capacitor filter clock signal is in Appendix D.

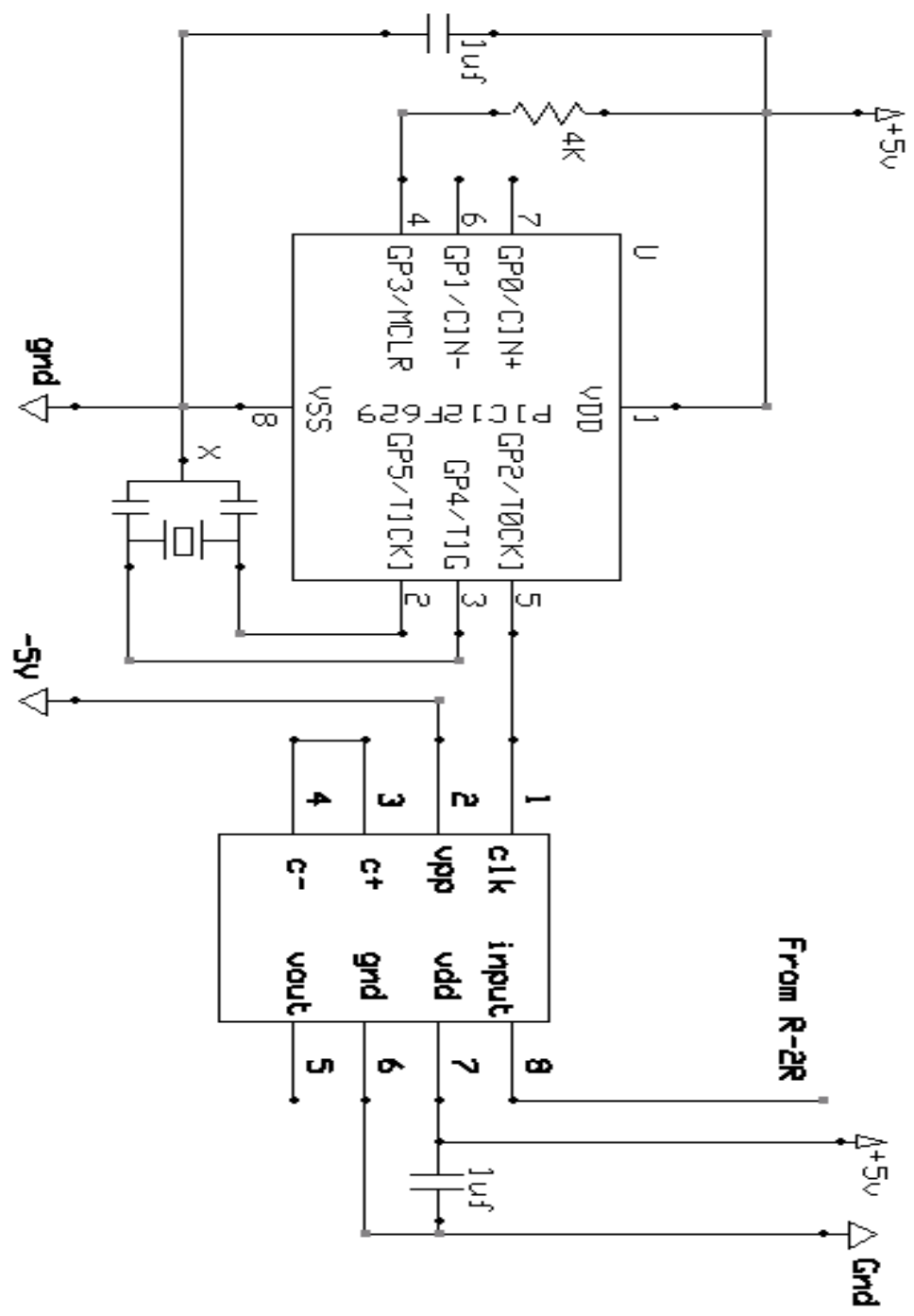

Figure 3.8.3 Smoothing Filter for R-2R Ladder 


\subsection{Detailed Schematic: Signal Conditioning Board}

The signal conditioning board contains amplifiers to scale two signals from the line out of the PC sound card or external microphones to the 0 to 5 volt range so they can be sampled by the analog two digital converters as shown in Figure 3.8.4.

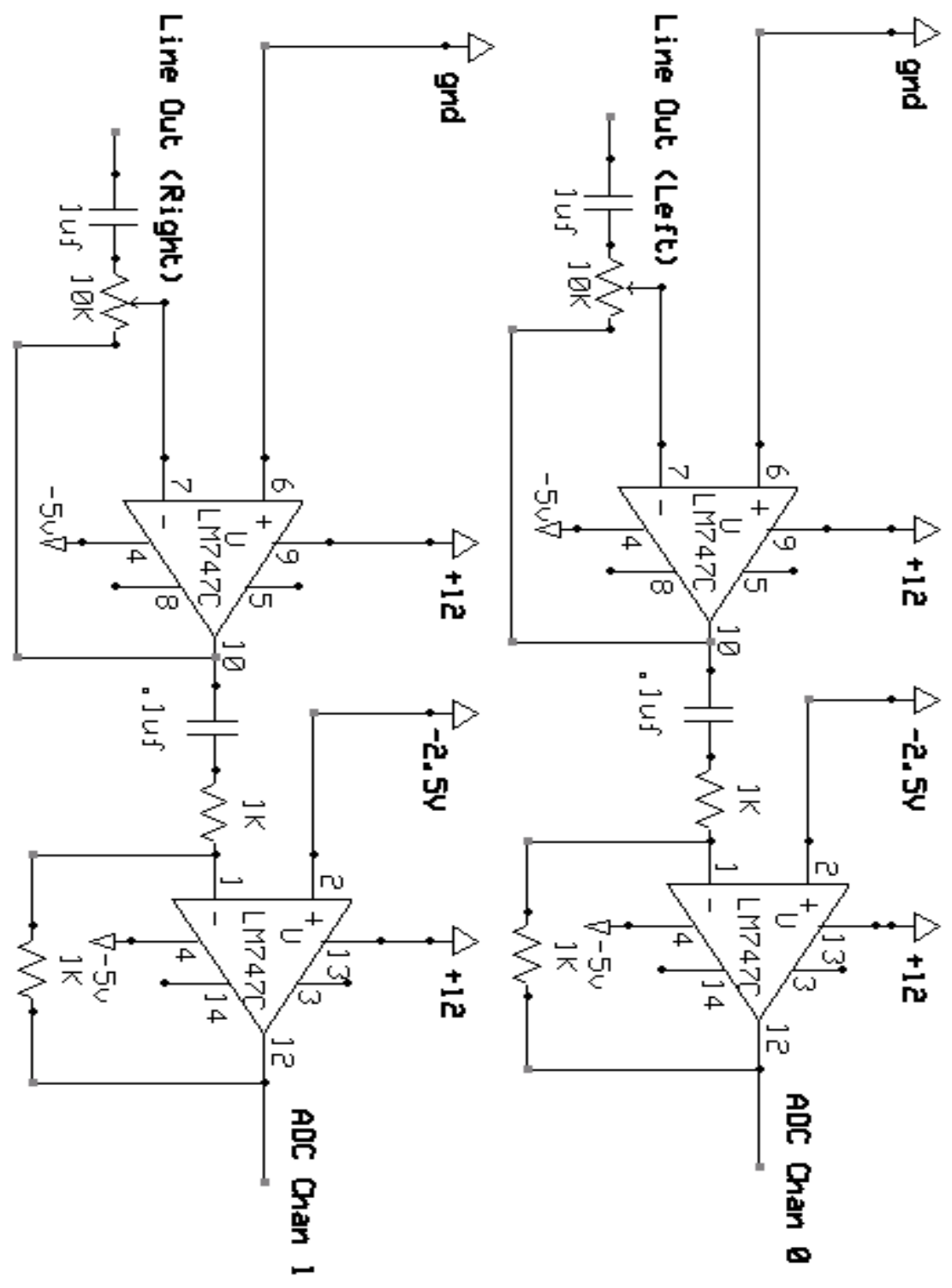

Figure 3.8.4 Signal Conditioning Block 


\subsection{Detailed Schematic: Signal Processing Board}

The signal processing board is built around the PIC18F452 chip. It contains the bare minimum circuitry that is required by the filter. The DAC uses a R-2R ladder to generate an output for convenience.

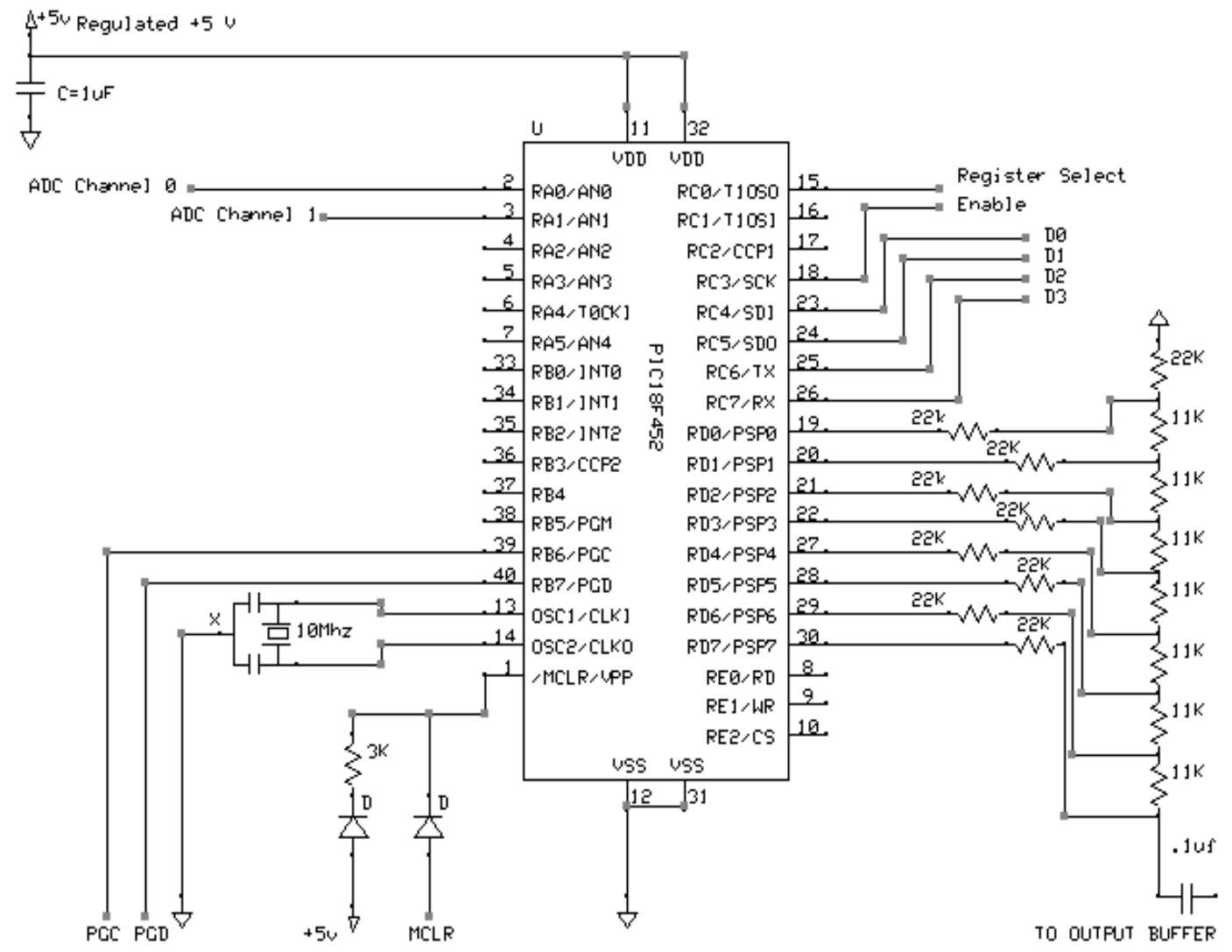

Figure 3.8.4 Block Overview of Circuit 


\subsection{Photograph of PIC18F452 based Filter}

This photograph of the test device, shown in Figure 3.8.5, was built to validate the filters designed and built during this project.

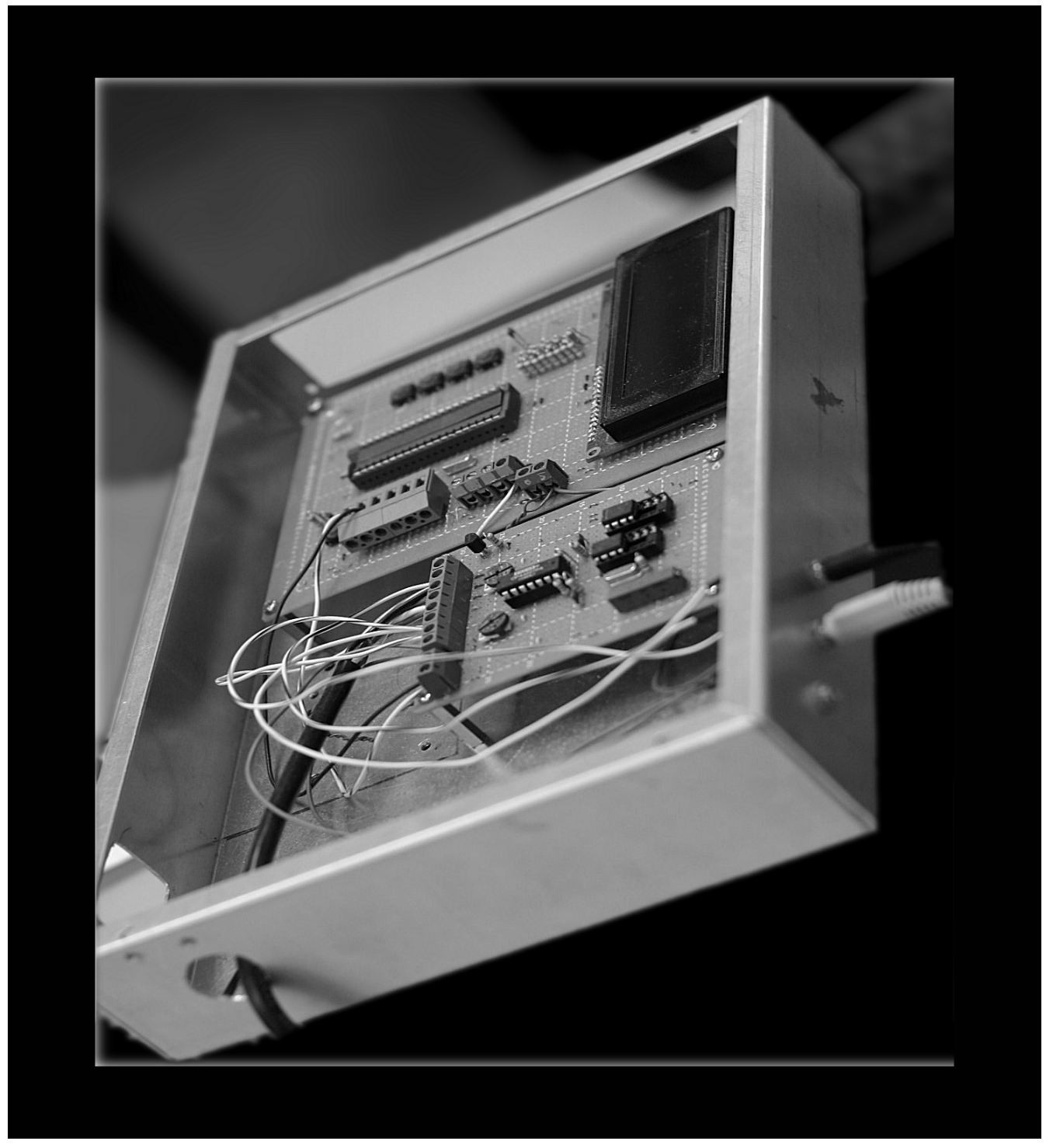

Figure 3.8.5 Photograph of Test Board 


\section{H A P T ER 4: RE S UL T S FR OM REA L-T IME}

All data acquisition was performed using a PC based audio processing program called Wavlab ${ }^{\text {TM }}$ Pro by Steinberg [14]. The program used the PC microphone input for data acquisition and contained a powerful set of visualization and analysis functions. This technique for data acquisition proved to be both elegant and efficient. The same software was used to generate various frequency sweeps that were used as input data to the PIC chip. LMS filters were also tested using the same apparatus.

\subsection{FIR FILTER: Data Acquisition Setup}

The apparatus setup for FIR filter testing is illustrated in Figure 4.1.1. In order to test the circuit with controlled waveforms, they were generated on a PC and send to the processing board via the line out of the sound card. The PIC board sampled the signals on the line out and after applying the processing LMS, send the DAC output back to the PC sound card, where it is sampled and stored as a wave file. This file is analyzed in WavLab ${ }^{\text {TM }}$ [14] and results are presented.

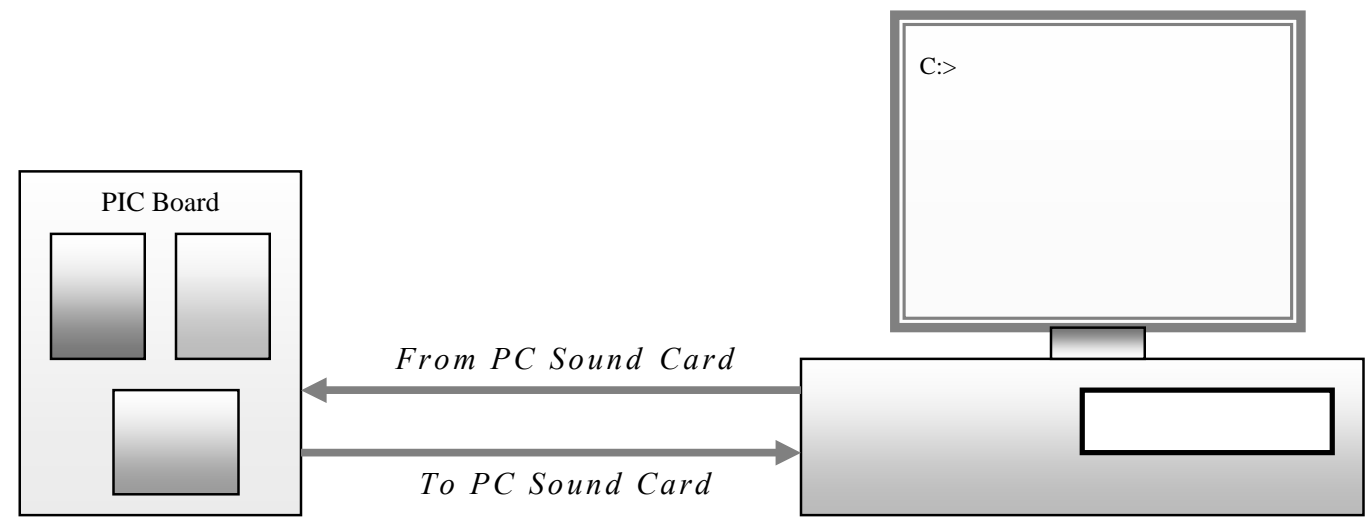

Figure 4.4.1 Basic setup for low-cost data acquisition 


\subsection{FIR FILTER: Real-Time Testing Results}

Several filter configurations were tested to validate and verify the operation of the FIR filter in the PIC chip. Matlab was used to generate filter coefficients (Taps) that were then transferred to the PIC chip.

\section{LOW PASS FILTER: Testing and Analysis}

The first test was a basic low pass filter with the following parameters. The filter illustrated in Figure 4.2.1 was generated with the PIC filter design software developed for MATLAB during the course of this research. The low-pass filter in Figure 4.2.1 was made with the desired attenuation of $50 \mathrm{~dB}$ in the stop-band $[0.6 \mathrm{~K}-1 \mathrm{~K}]$ with a $1 \mathrm{KHz}$ cutoff.
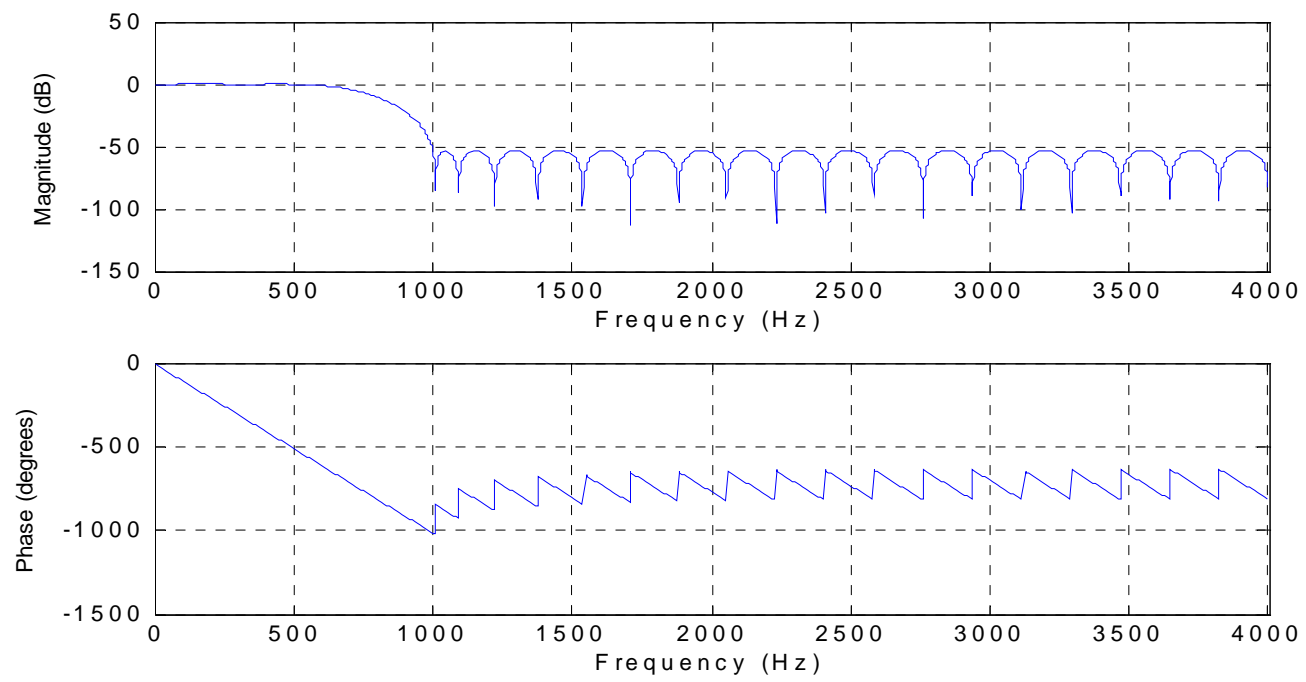

Figure 4.2.1 Response Curves of Intended Filter

The sampling frequency is $8000 \mathrm{~Hz}$ allowing the sampling of up to $4000 \mathrm{~Hz}$. Once the PIC was loaded with the firmware it was then tested using the test-signal shown in Figure 4.2.2. The test-signal is a constantpower frequency sweep 10 seconds long from [200 Hz to $4000 \mathrm{~Hz}$ ]. The sweep has constant amplitude in time domain and the frequency steadily 
increase from $200 \mathrm{~Hz}$ up to $4000 \mathrm{~Hz}$. Since the filter is designed to begin attenuation at $600 \mathrm{~Hz}$ and reach $50 \mathrm{~dB}$ at $1000 \mathrm{~Hz}$, the analysis of the test signal after running through the PIC filter should show how well the filter worked. Since this is a constant-power sweep the amplitude of the sweep attenuates with increasing frequency in as the FFT chart.

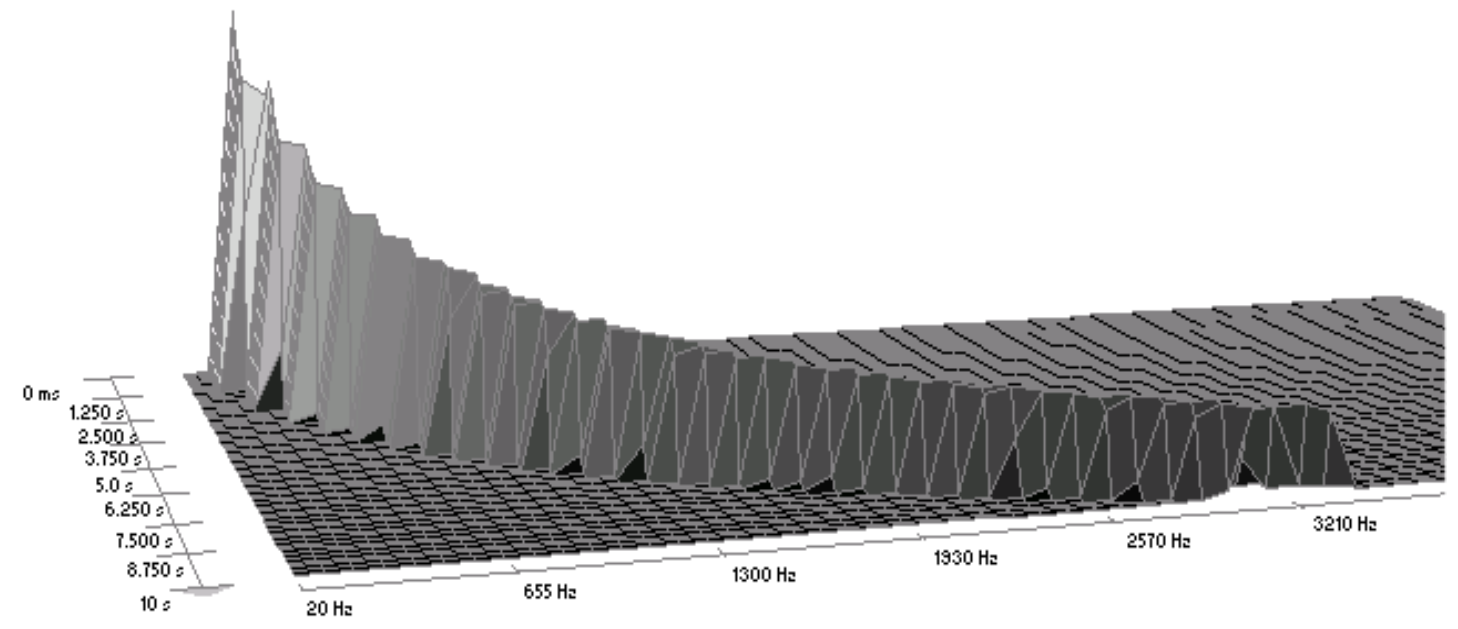

Figure 4.2.2 Test Signal: Constant Power Sweep [200Hz-4000Hz]

The frequency response of the output from the filter captured by a data acquisition system is shown in the Figure 4.2.3.

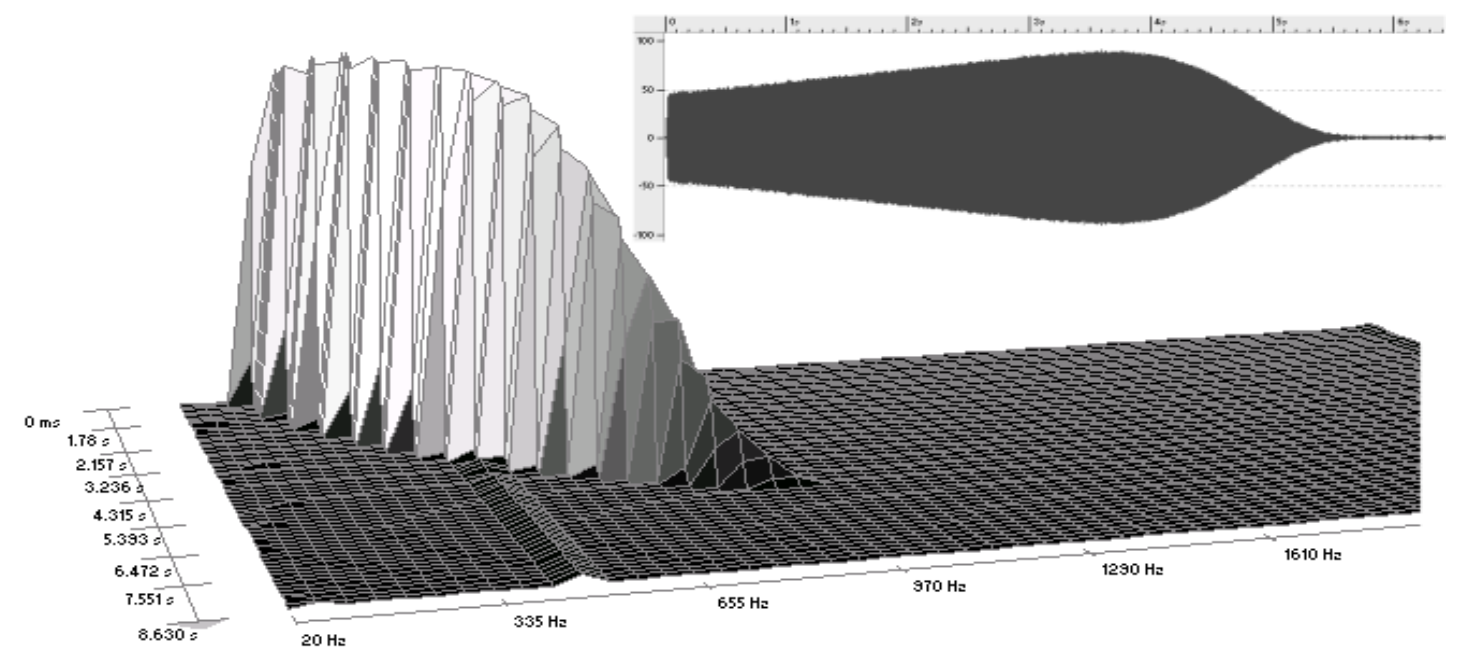

Figure 4.2.3 Filter Performance on PIC18F452 Chip 
The fast Fourier transform (FFT) of the DAC output shown in Figure 4.2.4 verifies the performance of the filter. The sweep does indeed begin attenuation from $600 \mathrm{~Hz}$ as desired and is almost entirely decimated at $1 \mathrm{Kz}$. In the time domain graph (also Figure 4.2.4) the amplitude of the sweep does indeed show attenuation in the high frequency side of the sweep. Notice the small attenuation in the low-frequency side of the sweep. This attenuation is not the result of the filtering in the PIC. This attenuation is due to a DC blocking capacitor in the PC sound card. Sound cards by design cannot sample DC and this is one of the drawbacks of not using an expensive PC based data acquisition systems.

\section{BAND STOP FILTER: Testing and Analysis}

The second filter generated by the filter design software was a band pass filter with the characteristics illustrated in Figure 4.2.4. Once a attenuation of $50 \mathrm{~dB}$ is desired in the stop-band $[600 \mathrm{~Hz}-1800 \mathrm{~Hz}]$. The sampling frequency remains $8000 \mathrm{~Hz}$.
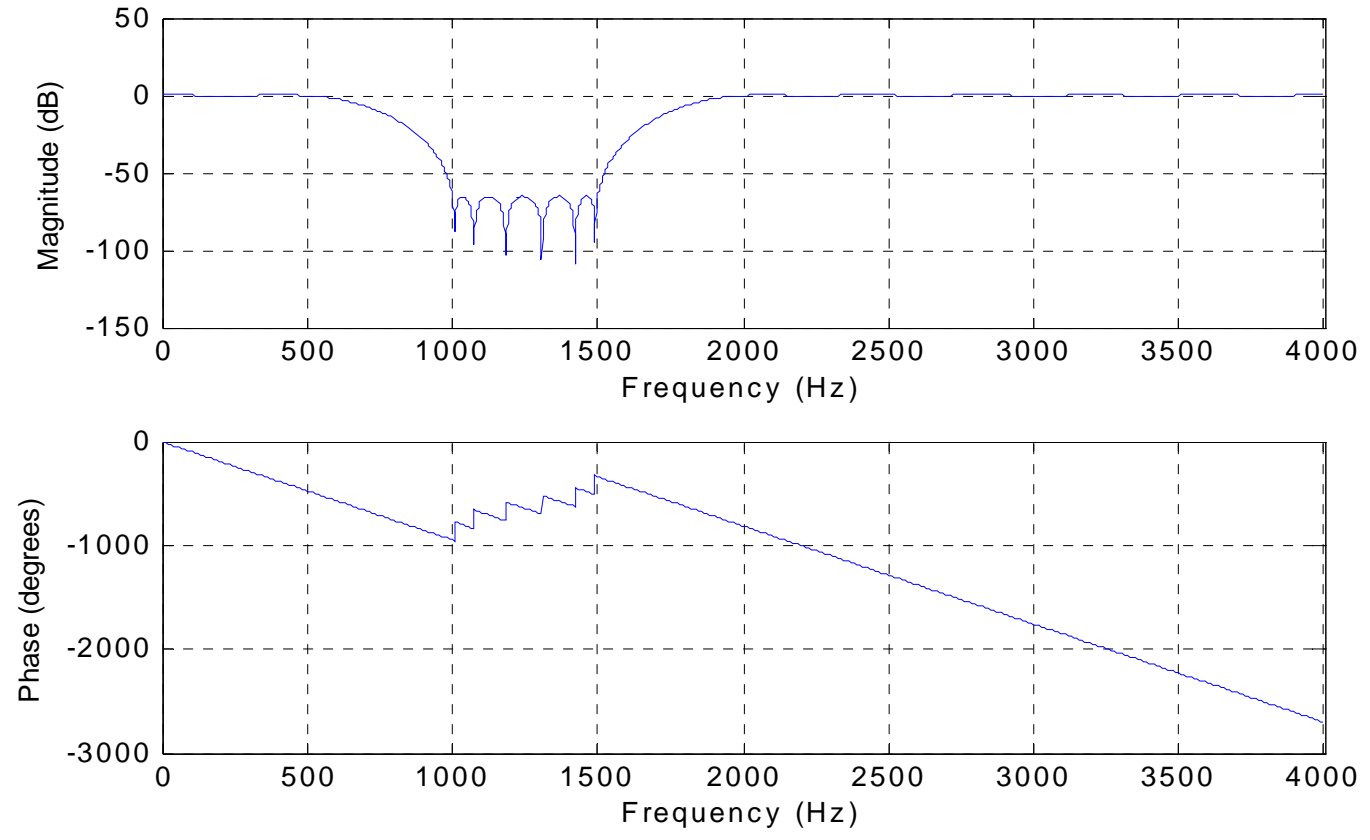

Figure 4.2.4 BSF Filter Specifications for Band Pass filter 
The FFT of the sampled data from the PIC chip illustrated in Figure 4.2.5 shows attenuation in the desired band $[600 \mathrm{~Hz}-1800 \mathrm{~Hz}]$.

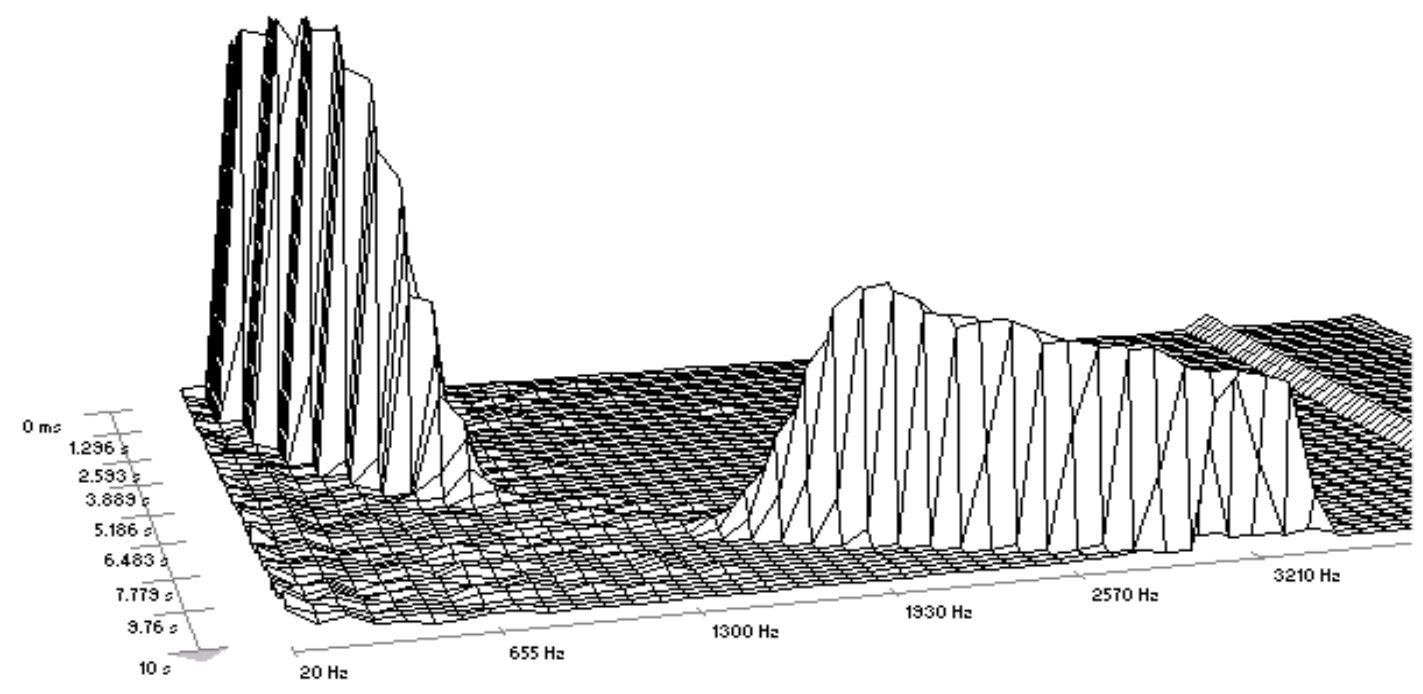

Figure 4.2.5 Measured BSF Frequency and Amplitude Response 


\section{MULTI-BAND FILTER 1 (FIR): Testing and Analysis}

The second filter that was implemented was a band stop filter. In Figure 4.2.6 a more complex multi-band FIR filter is shown. The filter has two stop-bands at $[600 \mathrm{~Hz}-1800 \mathrm{~Hz}]$ and $[2700 \mathrm{~Hz}-4000 \mathrm{~Hz}]$. Figure 4.2.7 shows the FFT of the PIC filter performance. Both stops bands have been attenuated as intended.
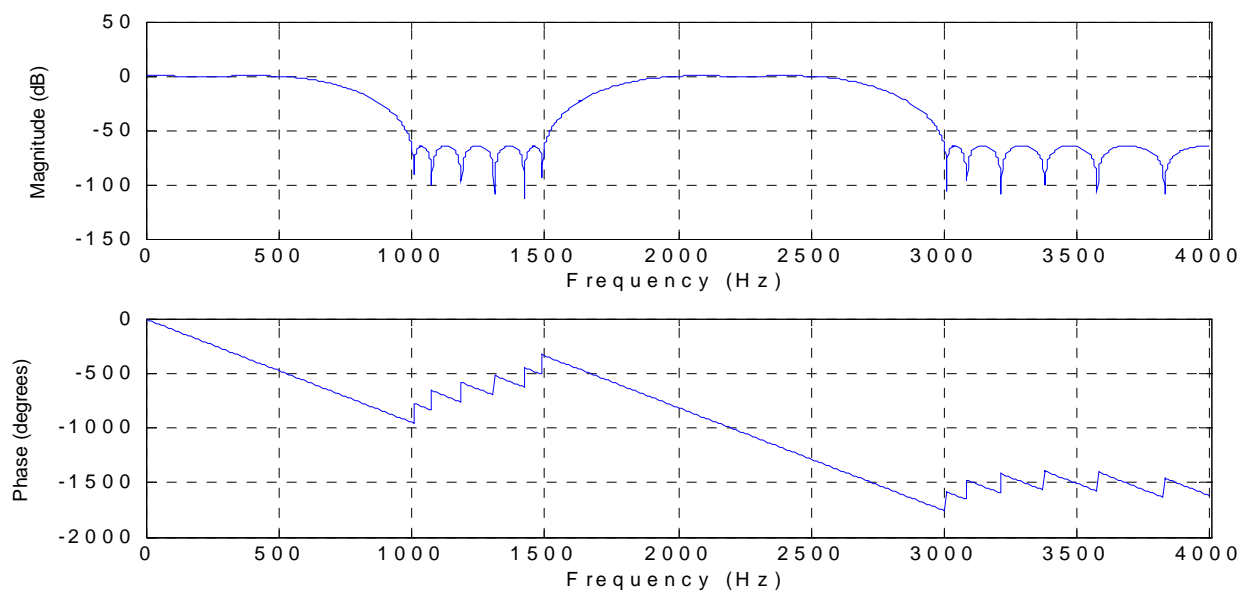

Figure 4.2.6 MBF 1 Filter Specifications for Band Pass filter

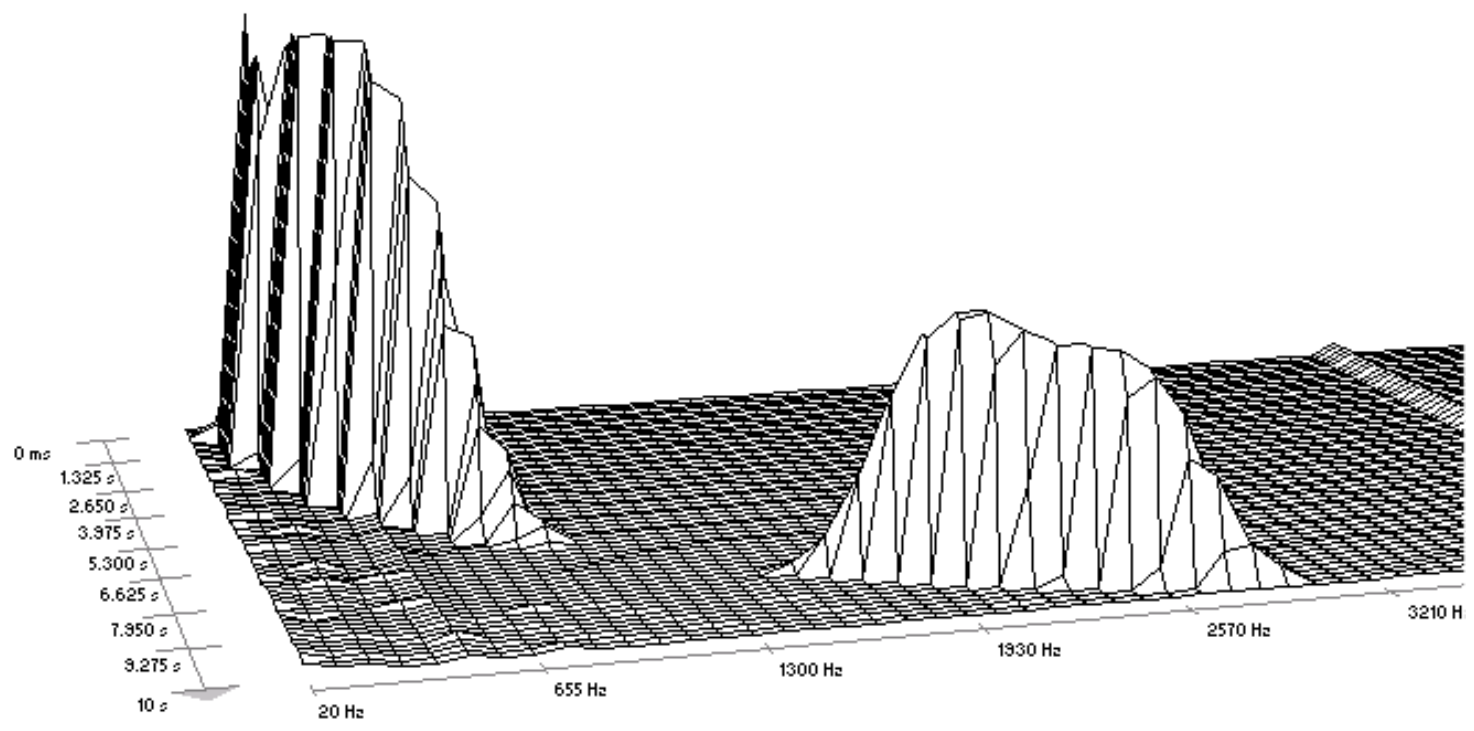

Figure 4.2.7 Frequency and Amplitude response from PIC18F452 


\section{MULTI-BAND FILTER 2 (FIR): Testing and Analysis}

Figure 4.2.8 is a filter with three pass-bands; Figure 4.2.9 shows the performance of the PIC chip. The figure shows that all three bands have been compensates as specified in Figure 4.2.8.
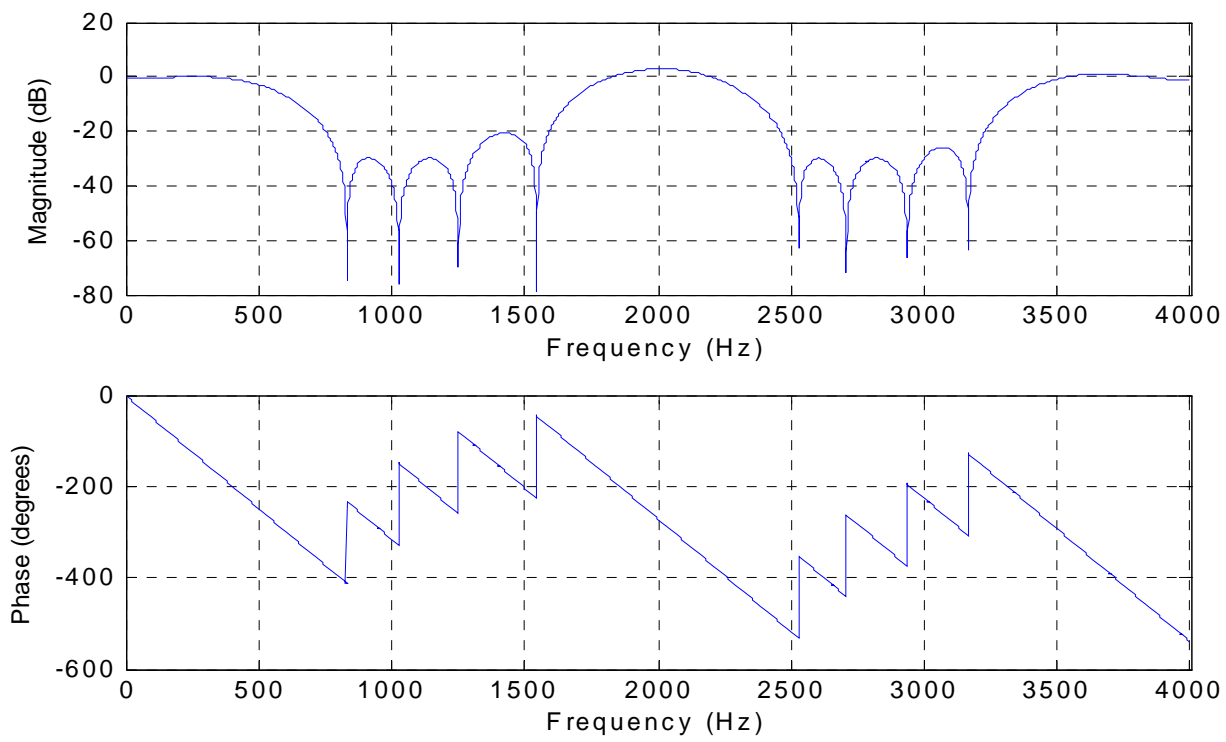

Figure 4.2.8 MBF 2 Filter Specifications for Band Pass filter

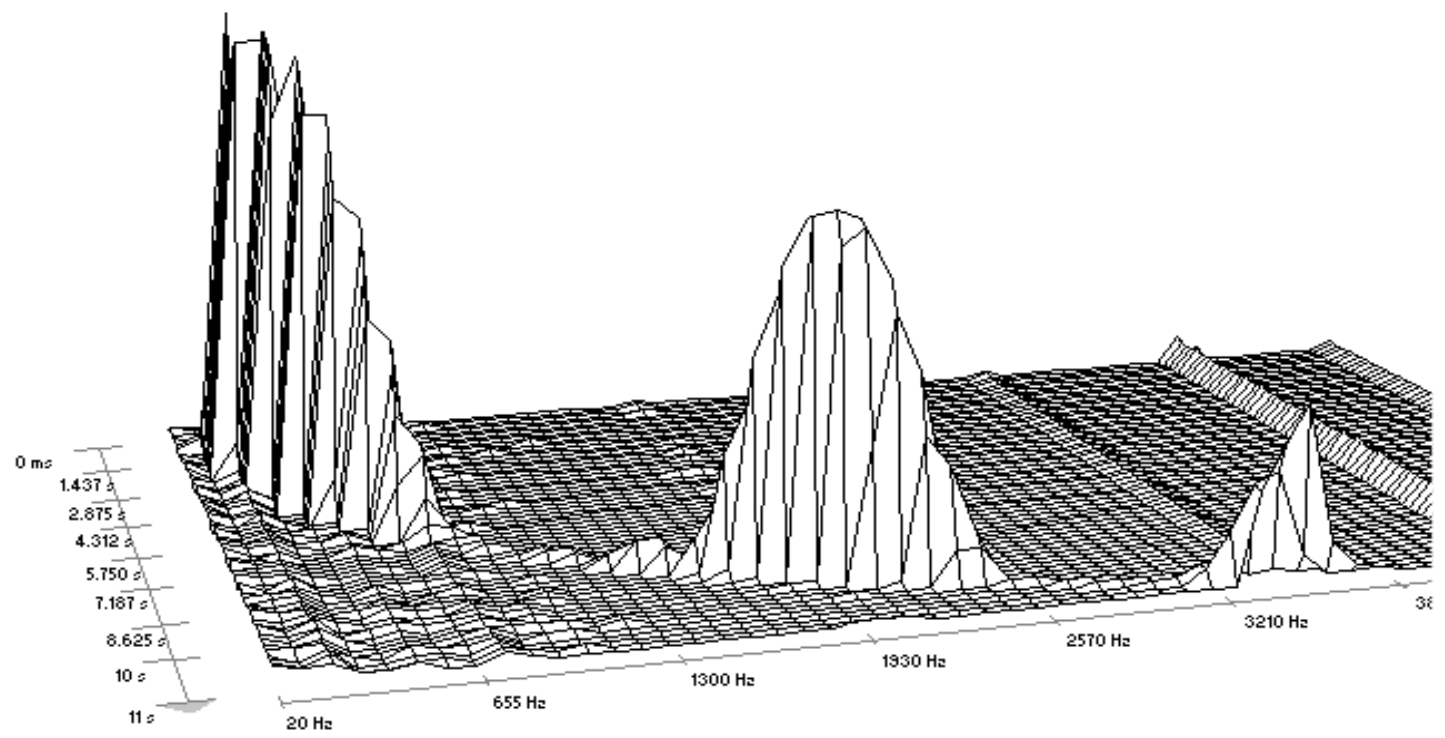

Figure 4.2.9 Frequency and Amplitude response from PIC18F452 


\subsection{LMS FILTER: Test Scenario 1}

In order to test the real-time adaptive filter a signal and noise vectors are carefully prepared. The LMS adaptive filter essentially applies the phenomenon of destructive interference to perform noise cancellation. Two waves can be successfully cancelled by destructive interference if they are both correlated in phase and amplitude. To illustrate the point made in the previous statement consider the scenario presented in Figure 4.3.1, the source $A$ produces a signal that is sampled at two points in space, p1 and p2. Even though at both points the signal is very similar however they cannot be directly subtracted because by the time the signal is sampled at p2 it is different in both phase as well as amplitude and cannot be simply destroyed by simple subtracting p1.

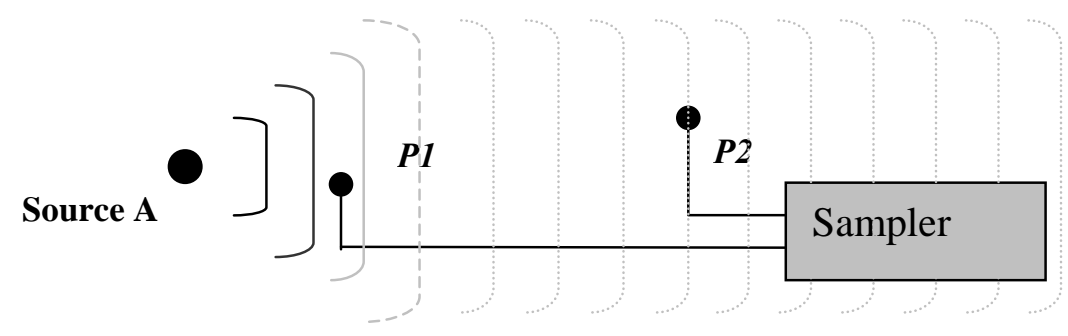

Figure 4.3.1 Sampling Source A at P1 and P2

To make the problem even more interesting a second source is added to the scenario presented in Figure 4.3.1 where a source B is introduced:

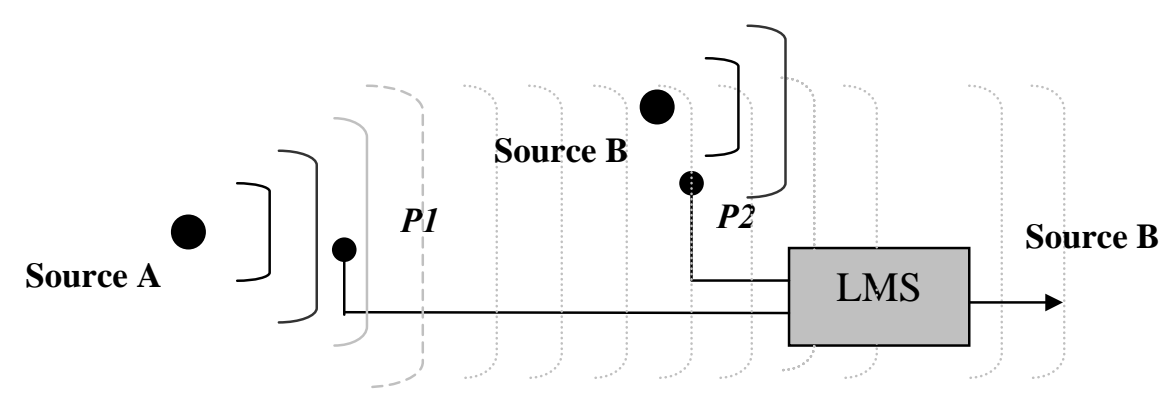

Figure 4.3.2 Source B is added to the scene 
Figure 4.3.2 shows that p2 will sample not only the signal from source B but also signal from Source A. The LMS adaptive filter has the ability to intelligently subtract Source A from source B by predicting the degree of contamination from Source $A$ in the sample of source B and recursively improving its' predictions until source A has been successfully eliminated from the sample made at p2. The test scenario uses two monotonic sin waves one for source A $(220 \mathrm{~Hz})$ and another for Source B $(340 \mathrm{~Hz})$. To simulate the effects of Source A traveling through space till sampling point p2 source $A$ is given a phase-shift of $375 \mathrm{us}$ (micro seconds) and a gain added to source B and the sum is normalized to approximate the signal sampled at p2. See Figure 4.3.3.

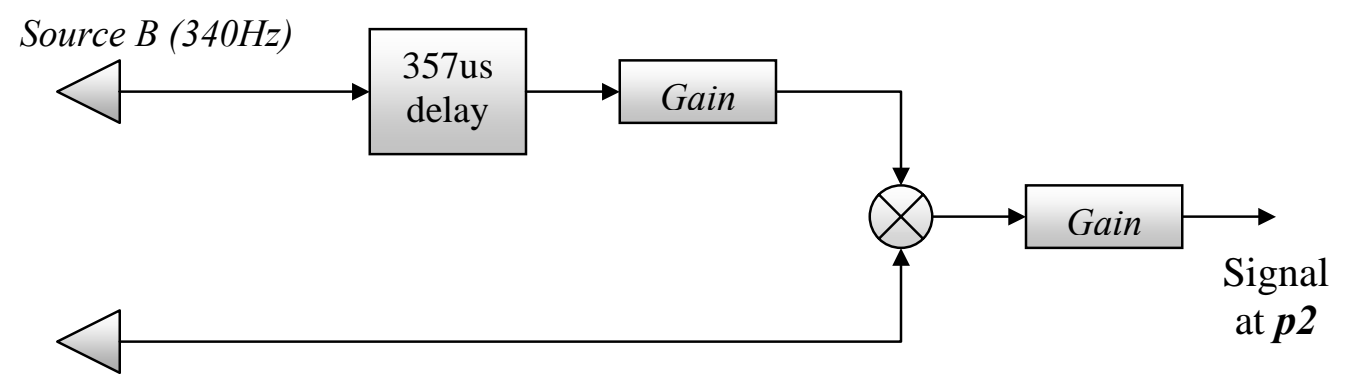

Source A (220Hz)

Figure 4.3.3 Approximation of Signal at p2

The signal used at $\mathrm{p} 2$ for the simulation was compiled in Matlab and a time domain graph is presented in Figure 4.3.4. This waveform is the superposition of source A and source B sampled at point p2. 


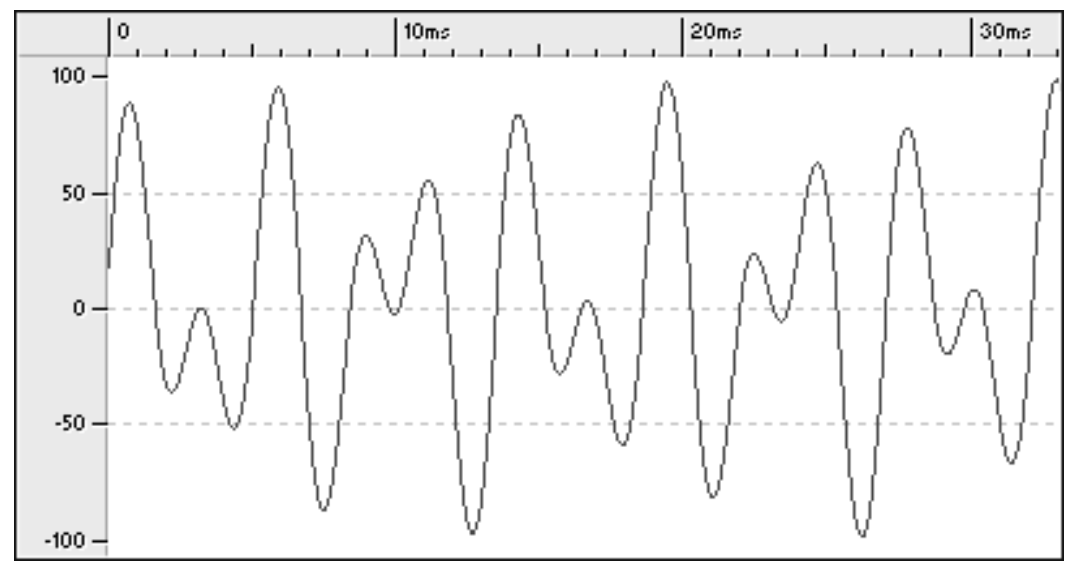

Figure 4.3.4 Time domain graph of signal at p2

Figure 4.3.5 shows the frequency domain representation of the signal sampled at p2. The two spikes are the two monotonic signatures of source A $(220 \mathrm{~Hz})$ and source $\mathrm{B}(340 \mathrm{~Hz})$.

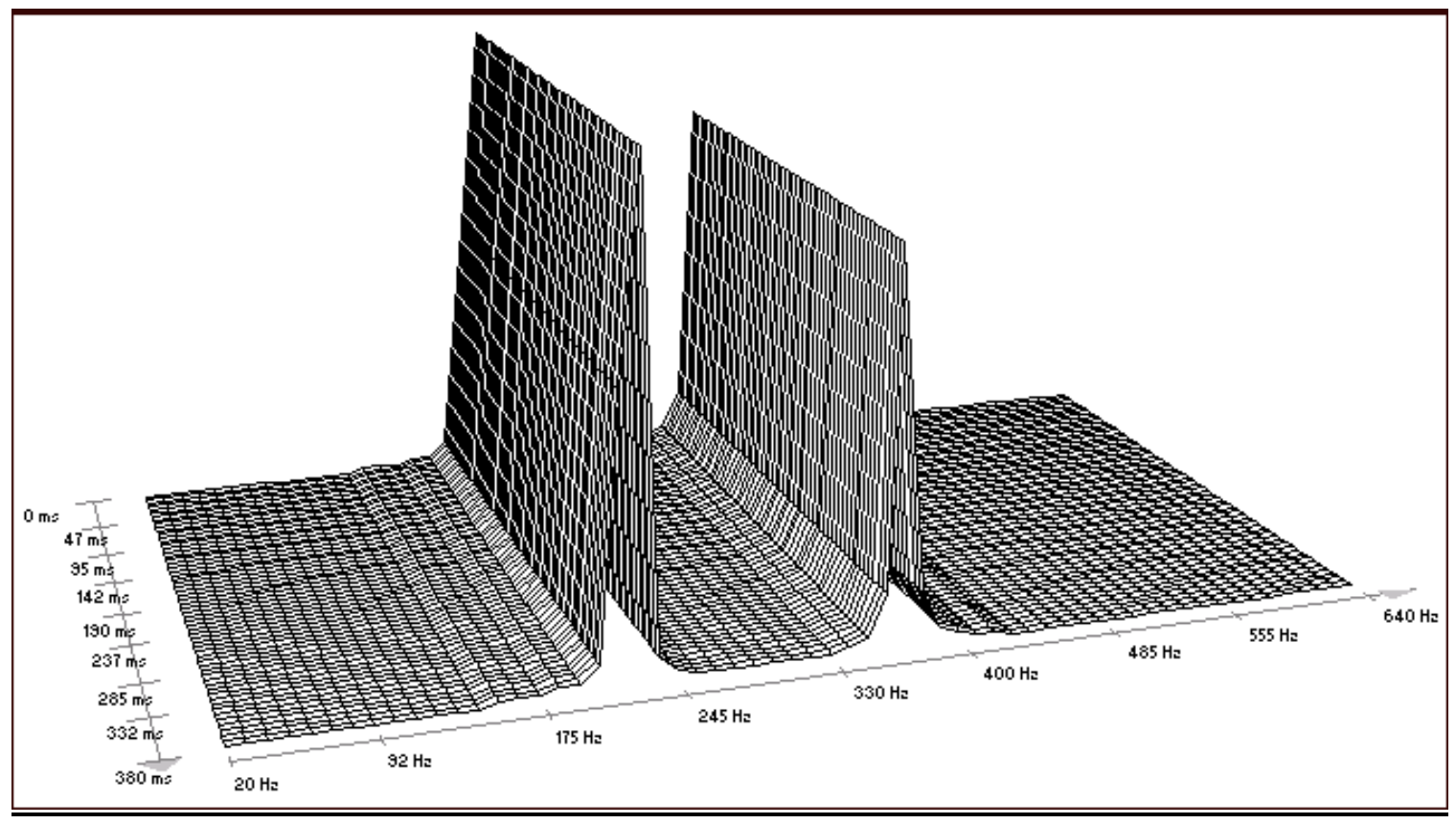

Figure 4.3.5 Frequency domain graph of signal at p2 
The real-time test topology is presented in Figure 4.3.6. The signal sampled at point p1 is approximated as source A $(220 \mathrm{~Hz})$ with a gain.

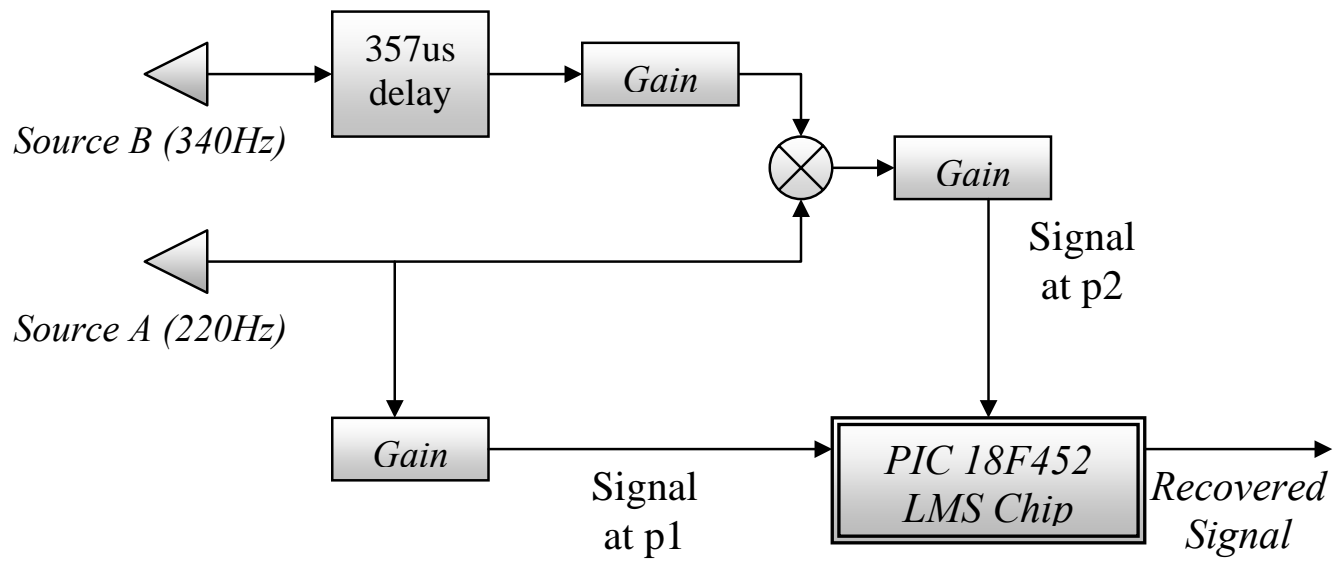

Figure 4.3.6 Real-time test topology

The signal sampled at point $\mathrm{p} 2$ and the signal at p1 (reference) going into the PIC chip are shown next in Figure 4.3.6 in time domain.

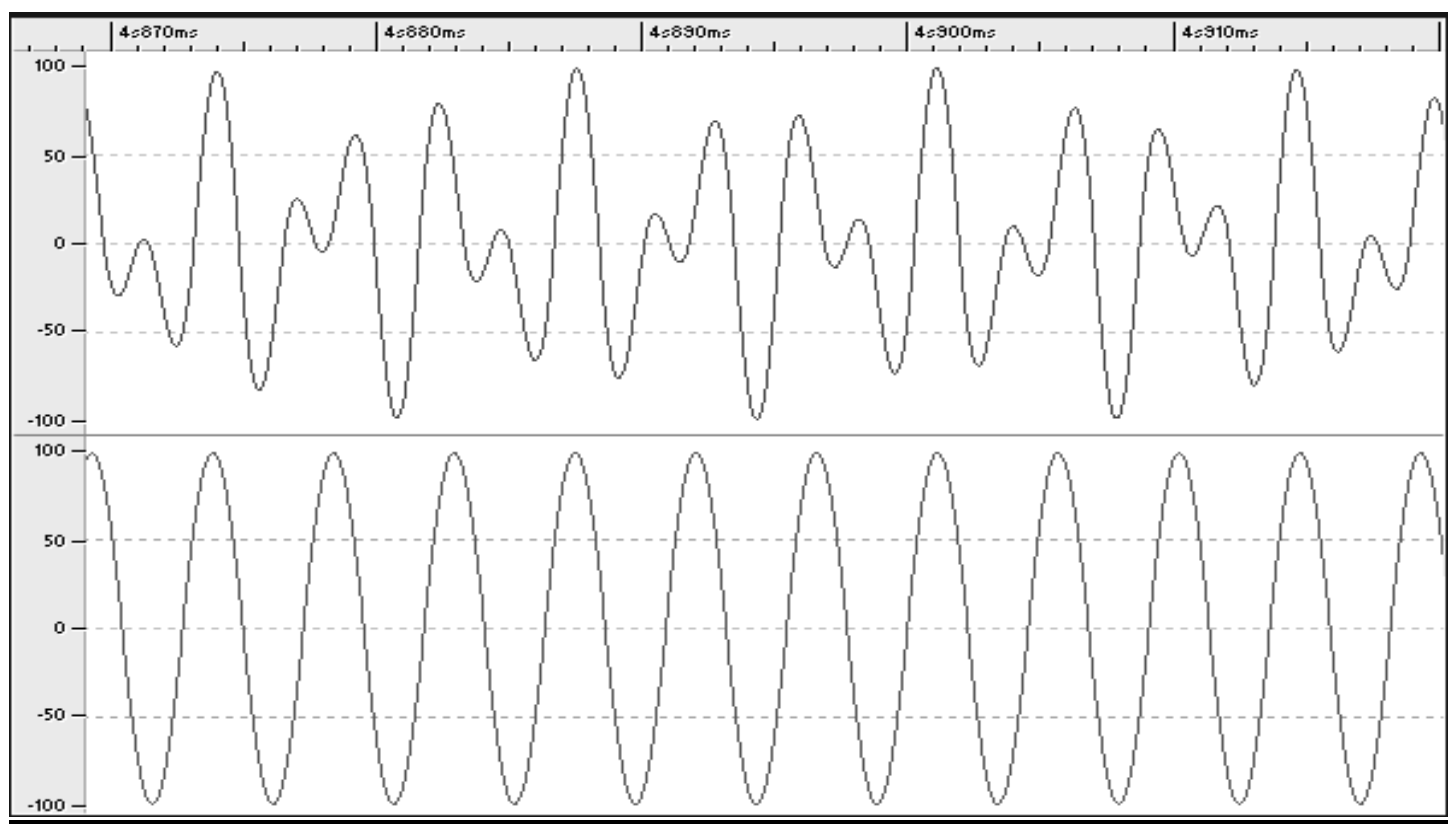

Figure 4.3.6 Signal p2 (top) and Reference p1 sampled by ADC 
The signal p1 and reference p2 are sampled by the PIC 18F452. The algorithm implemented in the PIC chip is a fourth order floating-point LMS with a sampling frequency of $8000 \mathrm{~Hz}$ and learning rate of 0.1 . Both signal and reference are simultaneously presented to the PIC chip as shown in Figure 4.3.5, the hardware setup for the experiment is the same as Figure 4.4.1. The output of the chip or the recovered signal is recorded and graphed in Figure 4.3.7 in time domain.

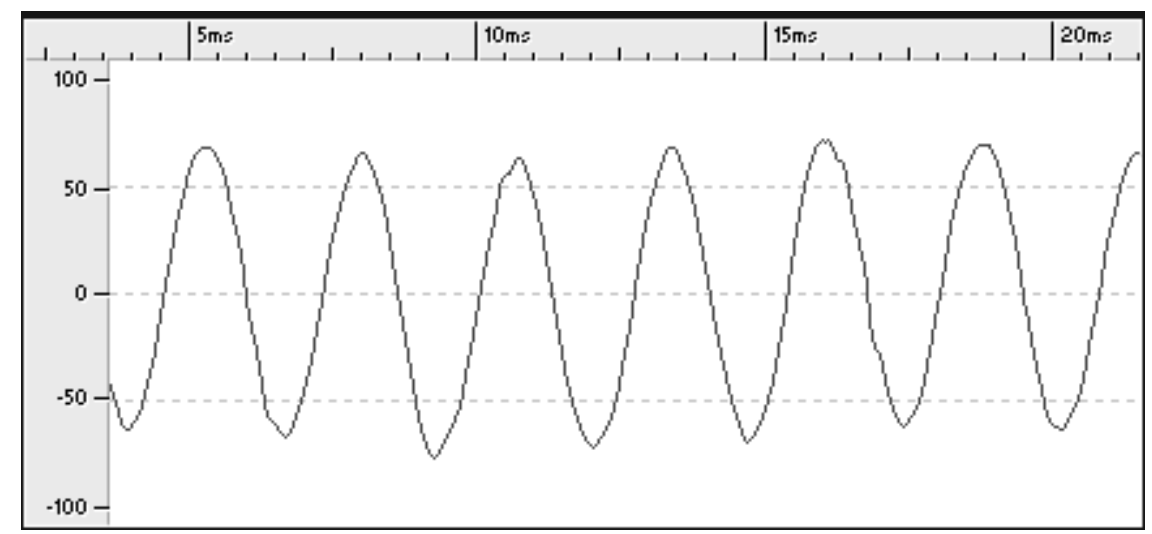

Figure 4.3.7 Signal Recovered by the PIC chop (source B: $340 \mathrm{~Hz}$ )

Figure 4.3.7 show that the LMS algorithm running in the PIC chip was indeed able to recover the Source B and the experiment was successful however, the lack of smoothness in the recovered signal suggests high-frequency noise. The smoothing-filter was given a cutoff of $4000 \mathrm{~Hz}$ however shifting that cutoff to a lower value will improve the signal to noise ratio. The frequency domain graph of the recovered signal is presented next in Figure 4.3.8. 


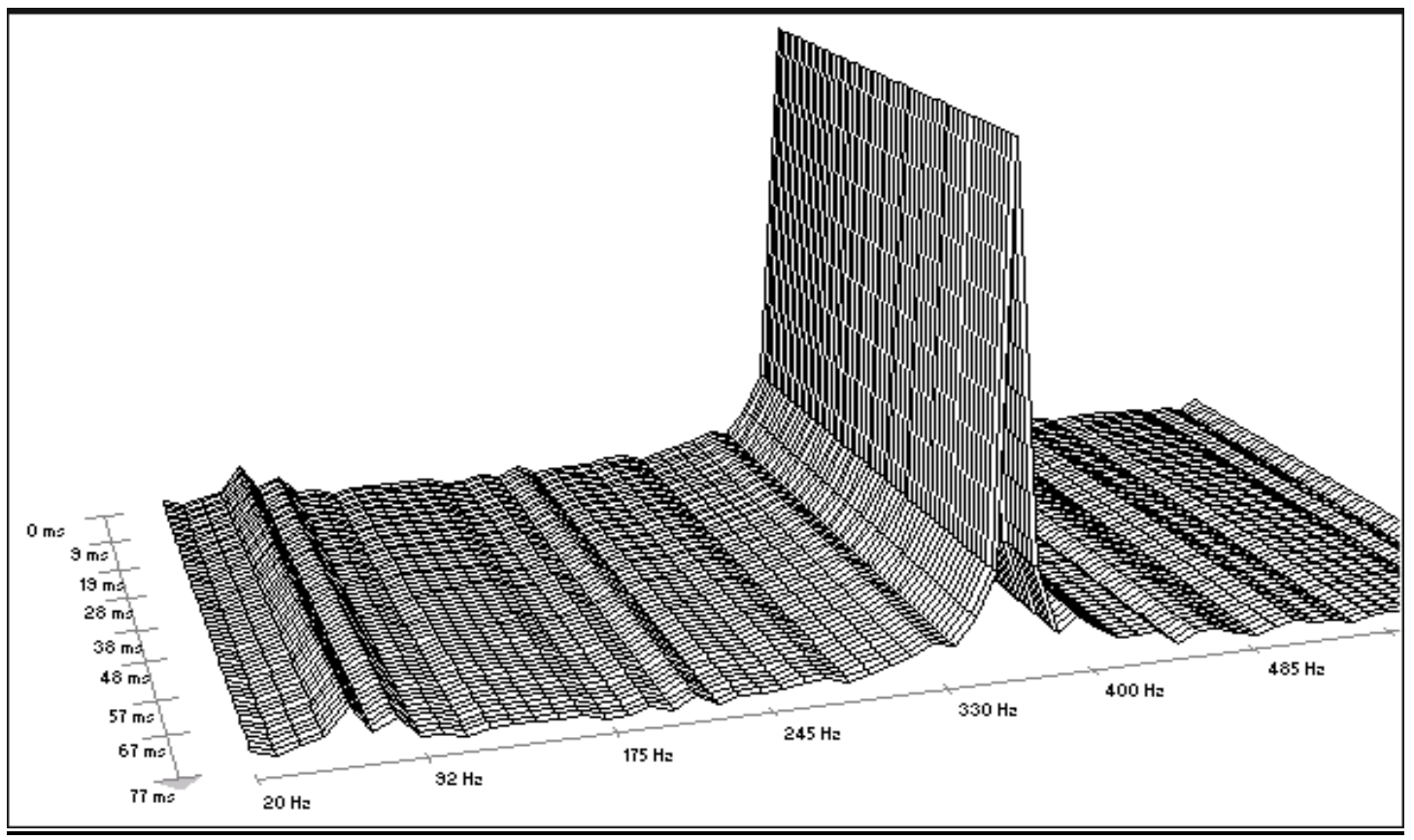

Figure 4.3.8 Signal Recovered in frequency domain (source B: $340 \mathrm{~Hz}$ ) 


\section{CHA P TER 5: ANALYSIS\& CONCLUSIONS}

Despite the hardware limitations of the PIC chip, both the FIR and LMS filters gave a strong performance with consistent, measurable and repeatable results.

\subsection{FIR filter performance summery}

The FIR filter attenuation requested in the filter presented in Section 4.2 is $50 \mathrm{~dB}$ in Matlab. Although the PIC chip faithfully reproduces the frequency response designed by Matlab, the attenuation of $50 \mathrm{~dB}$ could not be achieved. The best attenuation possible was $36 \mathrm{~dB}$.

The difference of $14 \mathrm{~dB}$ is attributed to the combined effect produced from two main factors. Firstly, the coefficients generated by Matlab are in double precision floating-point format, which were re-scaled into 8-bit fixed-point format numbers. This rescaling process is the major factor that contributes to the observed precision gap. The other factor is that the samples of the signal are made at 8-bit precision. In Matlab the test were made with the signal data sampled at 16 bits. The low bit depth in the sampled signal is also a factor that affects precision.

The best computation speed achieved is the theoretical minimum of $800 \mathrm{~ns}$ per MAC cycle by selecting the fastest execution-speed option. Equation 5.2 calculates the number of CPU cycles required to implement a FIR filter of a specified sized.

$$
\text { Num_cycles }(\text { taps })=8 * \text { taps }+45
$$


By using the smallest program size option, two additional instructions are added to the MAC loop thus increasing the size of the MAC cycle to 1000 ns. The Total cycles can is calculated using Equation 5.2.

$$
\text { Num_cycles }(\text { taps })=10 * \text { taps }+45
$$

By using the smallest RAM size option, the size of each MAC loop is extended further to 22 cycles per MAC. Thus the total number of cycles used by the PIC chip is calculated using Equation 5.3.

$$
\text { Num_cycles }(\text { taps })=22 * \text { taps }+45
$$

Equation 5.4 determines RAM usage for a given filter order for fastest-execution speed option.

$$
\text { Ram_needed }(\text { taps })=2 * \text { taps }+8
$$

Equation 5.5 determines RAM usage for a given filter-order in the minimum RAM implementation case:

$$
\text { Ram_needed }(\text { taps })=\text { taps }+8
$$

The execution speeds from all three available implementation models are plotted and shown in Figure 5.1. 


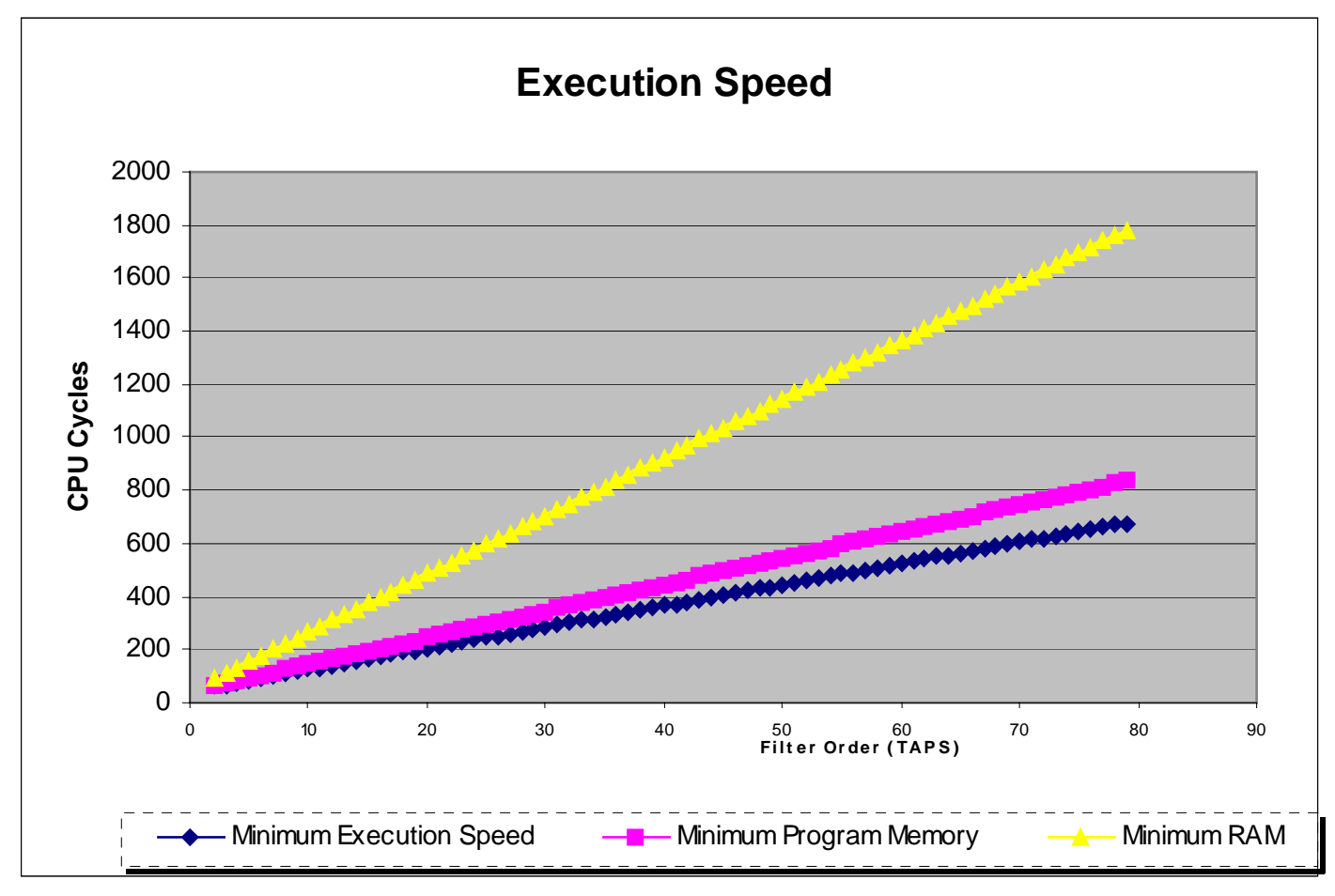

Figure 5.1 Comparison of execution speed for different implementations

\subsection{LMS filter performance summary}

One of the intentions of this research was to obtain a reasonable approximation of how many orders can be achieved on the PIC 18F452 chip given it's many limitations. Using the floating-point library and best speed achieved for the adaptive filter is 267 cycles per tap.

At 10 million instructions per second (MIPS), achievable with a $10 \mathrm{MHz}$ external crystal oscillator, the PIC chip can execute 267 cycles in 26.7 $\mu \mathrm{s}$. Equation 5.6 estimates the highest achievable order for a specified sampling rate and Equation 5.7 estimates the max sampling frequency for the specified number of taps. 


$$
\begin{aligned}
& \text { Max_LMSOrder }(\text { sampling _freq })=\text { floor }\left\{\frac{\text { ext_osc }}{4 * \text { sampling_freq } * 267}\right\} \\
& \text { Max_LMS_Sampling _freq }=\frac{\text { ext_osc }}{4 * \text { LMSOrder } * 267}
\end{aligned}
$$

Table 5.1 shows the relationship between Sampling frequency and the maximum filter order achievable using the implementation strategy developed during this research. The number of Taps cannot exceed 375 because the PIC will run out of RAM.

Table 5.1: LMS Sampling Rate vs. Taps

\begin{tabular}{|cc|}
\hline Sampling Freq $\mathbf{( H z )}$ & Taps \\
100 & 375 \\
200 & 187 \\
400 & 94 \\
800 & 47 \\
1600 & 23 \\
3200 & 12 \\
6400 & 6 \\
12800 & 3 \\
25600 & 1 \\
& \\
External Clock & 40000000 \\
\hline
\end{tabular}

\subsection{Conclusions and future work}

The following conclusions were draws regarding the various filter implementations that were explored during the course of this research.

1. The PIC 18F452 chip is an excellent candidate for fixed-point FIR filter implementation. At 800 ns per MAC cycle, there is no obvious disadvantage to diverting part of the PIC CPU cycles. 
2. No more than a fourth, order LMS filter is possible using the floating point system using the PIC 18 family. Higher orders are possible at lower sampling rates, however the lack of the normalization operation in the floating-point variables makes it prone to loss of precision from roll-off errors. The newer chips in the same class and price bracket, such as the dsPIC family, contains specific hardware such as a 40 bit barrel shifter, 16-bit signed multiplier and 16 bit ALU with speeds up to 30-40MIPS. These chips overcome many of the hardware limitations of the PIC 18 family, making them highly suited for building practical applications of adaptive filters, neural networks etc without having to resort to using the cost prohibitive DSP boards. Future work can include developing programming tools and software libraries for this new family of chips.

3. In the LMS filter developed during this research is not used in any particular applications. It was merely evaluated and tested on simulated data and meant as to be a resource that can be applied to a specific application. There is scope for finding a suitable application for this filter such as adaptive noise cancellation headphones, standing wave decimation, line echo cancellations etc. 


\section{BIBLIOGRAPHY}

[1] Ananda Mohan P.V, Ramachandran V., Swamy M.N.S, Switch capacitor filters: Theorm, Analysis and Design, Prentice-Hall PTR, June 1995.

[2] CCS compilers, www.ccsinfo.com/picc.shtml, 2004

[3] CCS Compilers, C Compiler Reference Manual, Custom Computer Services Incorporated, Brookfield WI, 2003.

[4] Digikey, www.digikey.com, 2003

[5] Emmanuel C. Ifeachor, Barrie W. Jervis. Digital Signal Processing: A practical Approach, Addison-Wesley Publishing Company, 1993.

[6] Hall V. Douglas, Microprocessors and Interfacing programming and hardware, Glencoe McGraw-Hill, New York, New York, 1997.

[7] Hamming, R. W. Digital Filters third edition, Dover Publications, INC. Minneola, New York, 1989.

[8] Karam L. J, McCellah, J.H., Design of optimal digital filters with arbitrary magnitude and phase responses. IEEE International Symposium on Circuit and Systems. Circuits and Systems connecting the World, 1996.

[9] Lathi B. P. Signal Processing and Linear Systems, Berkeley Cambridge Press, Carmichael, California, 1998.

[10] Microchip, PIC18FXX2 Data Sheet: High Performance, Enhanced FLASH Microcontrollers with 10-Bit A/D, 2002.

[11] Predko Mike, Programming and customizing PICmicro ${ }^{\circledR}$ Microcontrollers, McGraw-Hill, New York, New York, 2002. 
[12] Ramu Anantha B. K. "Implementation of FIR and IIR Digital Filters Using PIC18 Microcontrollers”, Microchip Application Note: AN853, Appendix A, 2002.

[13] The Math works, Filter Design Toolbox 2 for designing and analyzing advanced floating-point and fixed-point filter. www.mathworks.com/products/filterdesign, 2003.

[14] Widrow B. and Winter R. Neural nets for adaptive filtering and adaptive pattern recognition. IEEE Computer, 1998. 
APPENDIX A

USERS MANUAL FOR FILTER DESIGN SOFTWARE

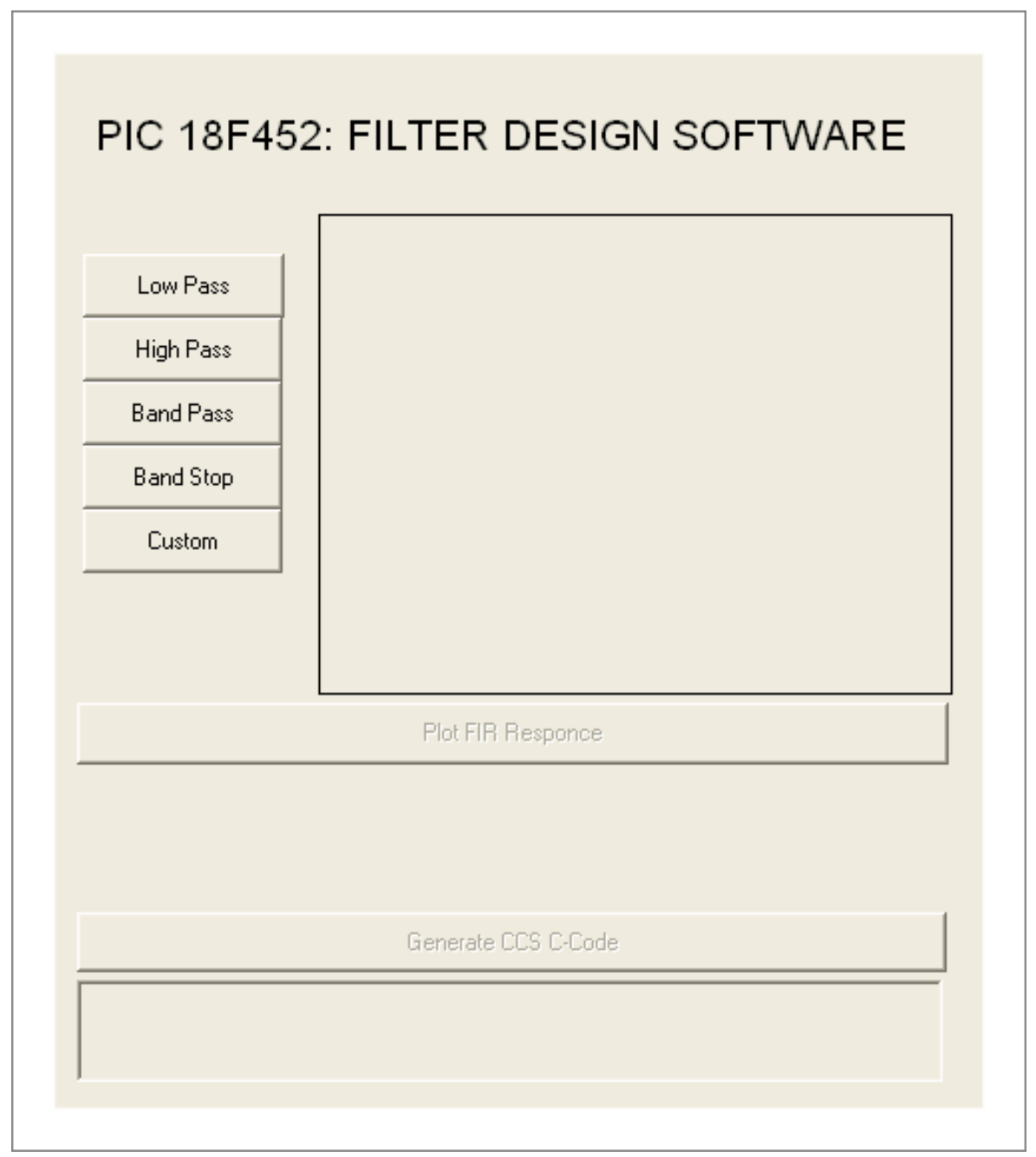




\section{INTRODUCTION}

The PIC18F452: FILTER DESIGN SOFTWARE was built on the Matlab environment and will only operate on MATLAB Version 6.1.0.450 (R12.1) and up. Filter Design Toolbox Version 2.1 must also be installed within the MATLAB environment.

In order to begin the filter design system a path must be set to the directory in which the source files are held. There are two ways of setting the path to the correct directory.

Method1: The path can be set directly by entering it on the provided space on the main tool bar or by clicking on the $\ldots$. button.

$?$ Current Directory: CMALTLABEp1 Work

Method2: An alternate way to set the directory path is to use the command line option in the main window of Matlab.

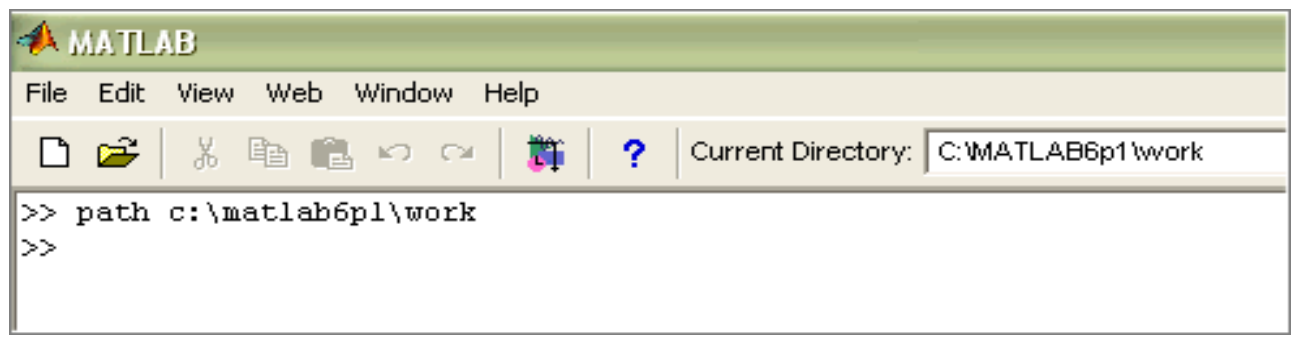

Once the path has been set the filter design system can be launched by typing in 'fildes' at the command prompt.

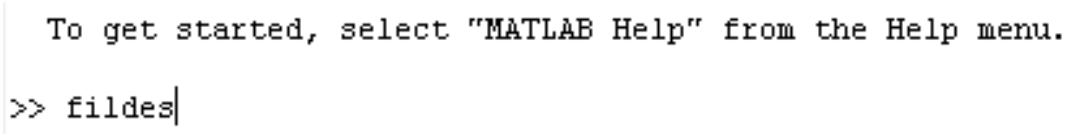


The filter design system main window offers the following functions, low-pass filter design, high-pass filter design, band-pass filter design, band-stop filter design and custom filter design.

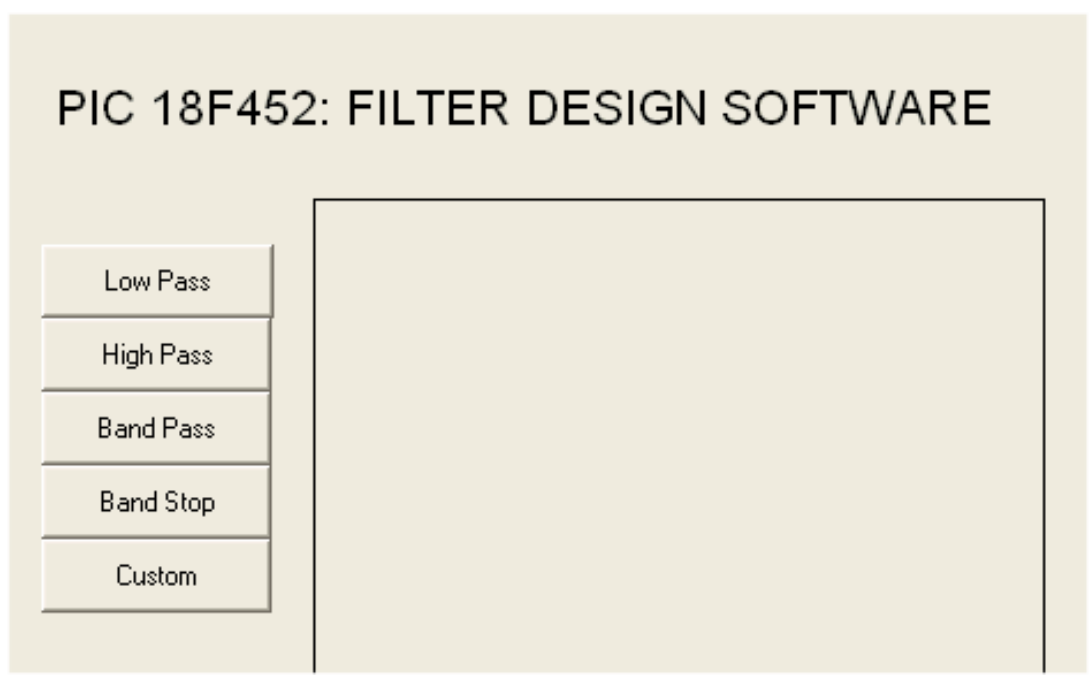

\section{LOW PASS FILTER DESIGN}

Clicking on the Low Pass $\mid$ button in the main window and enables the low-pass filter design interface where the parameters for the intended low-pass FIR filter can be entered.

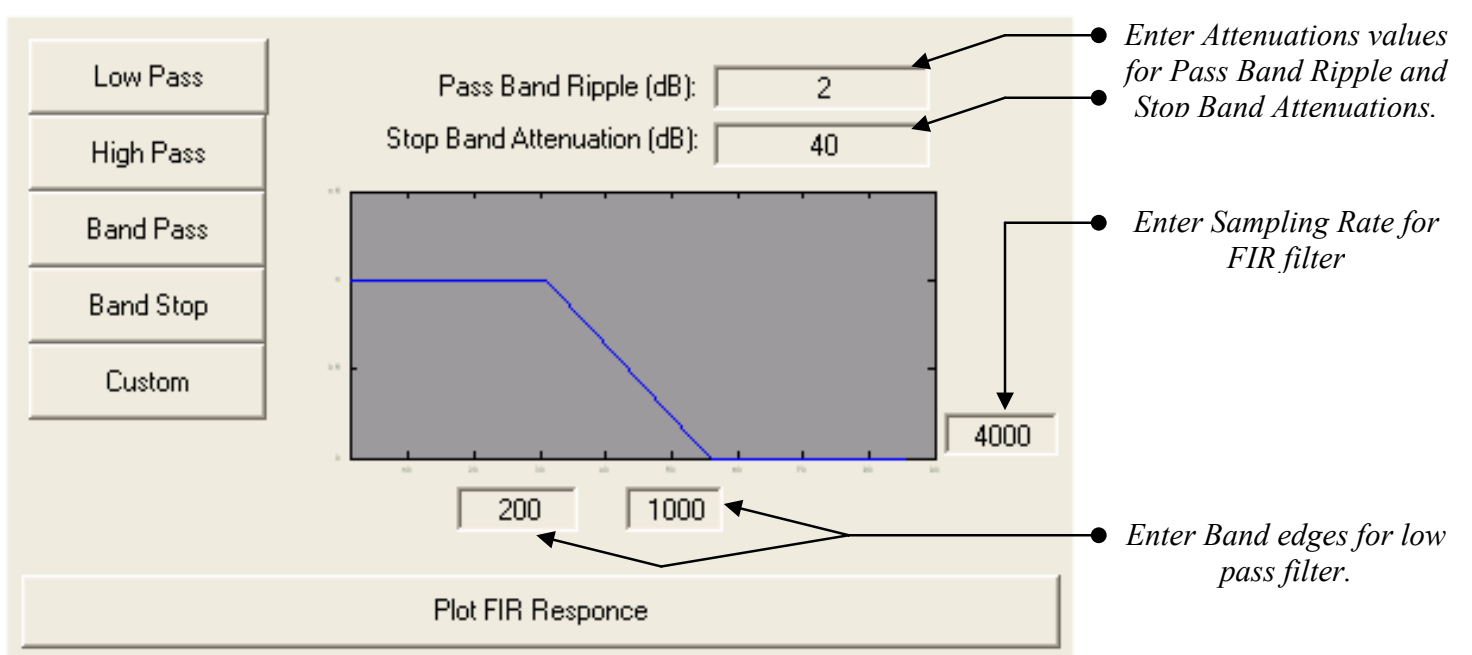


Once the desired band-edges and attenuations have been entered, by pressing the

Plot FIR Responce

simulated filter response is plotted to the screen.

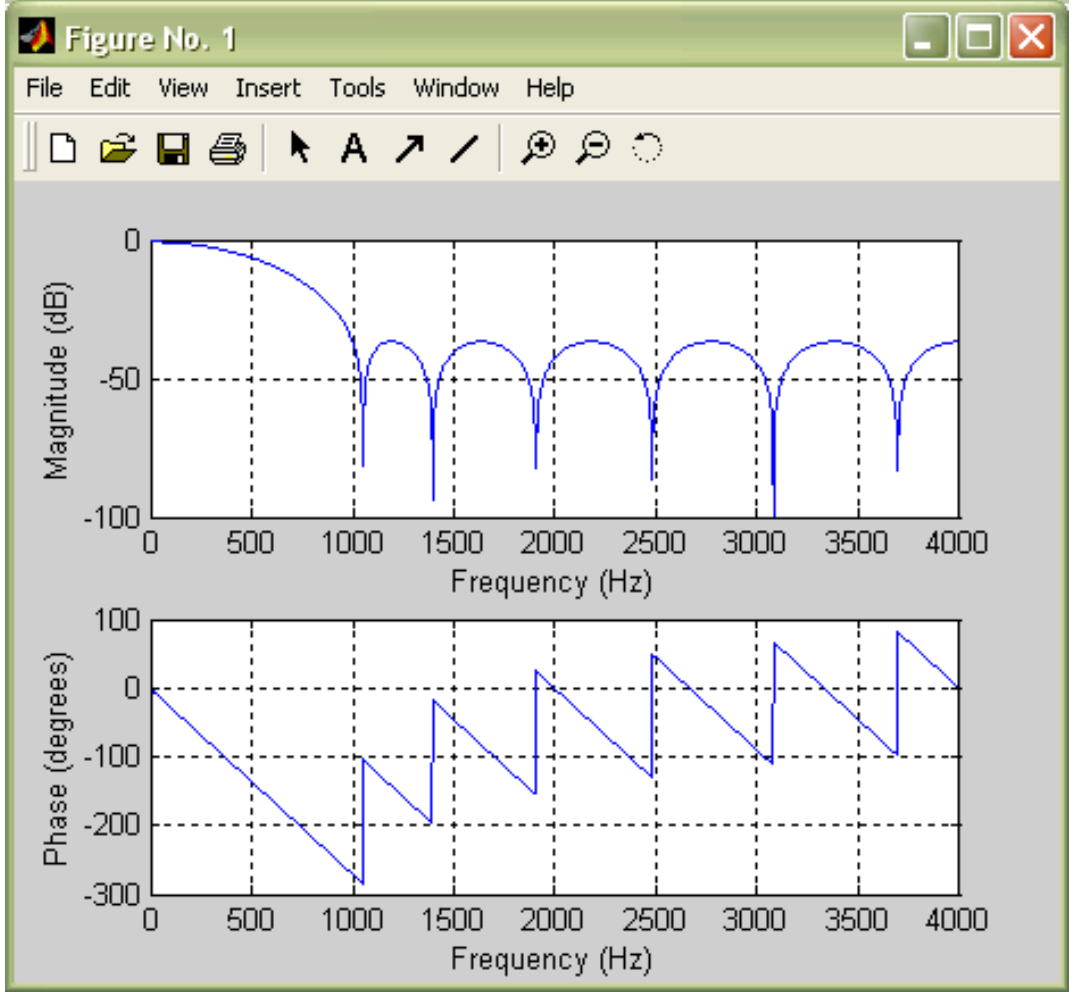

The frequency response and the phase response curves are graphed for user inspection. Additionally new options appear in the main window of the LPF design interface.

\section{Plot FIR Responce}

C. Fastest Execution 149 cycles, 34 Bytes RAM used

C Small Program 175 cycles, 34 Bytes RAM used

C Best Memory 334 cycles, 21 Bytes RAM Used

\section{Generate CCS C-Code}

Number of Taps Needed: 13, Type: 1 
The new items that appear in the interface present three implementation options. Each option lists the bytes of RAM used by the program on the PIC Chip as well as the number of cycles used by the program. Running at 40,000,000 each cycle lasts 100ns. Finally the Generate CCS C-Code button will generate the c-language file that can be installed into the PIC chip.

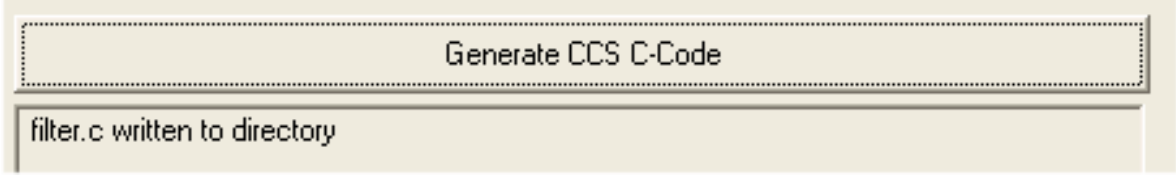

\section{HIGH PASS FILTER DESIGN}

The high-pass FIR filter is designed in the same way as the low-pass filter by selecting the 'High Pass' button on the main menu.

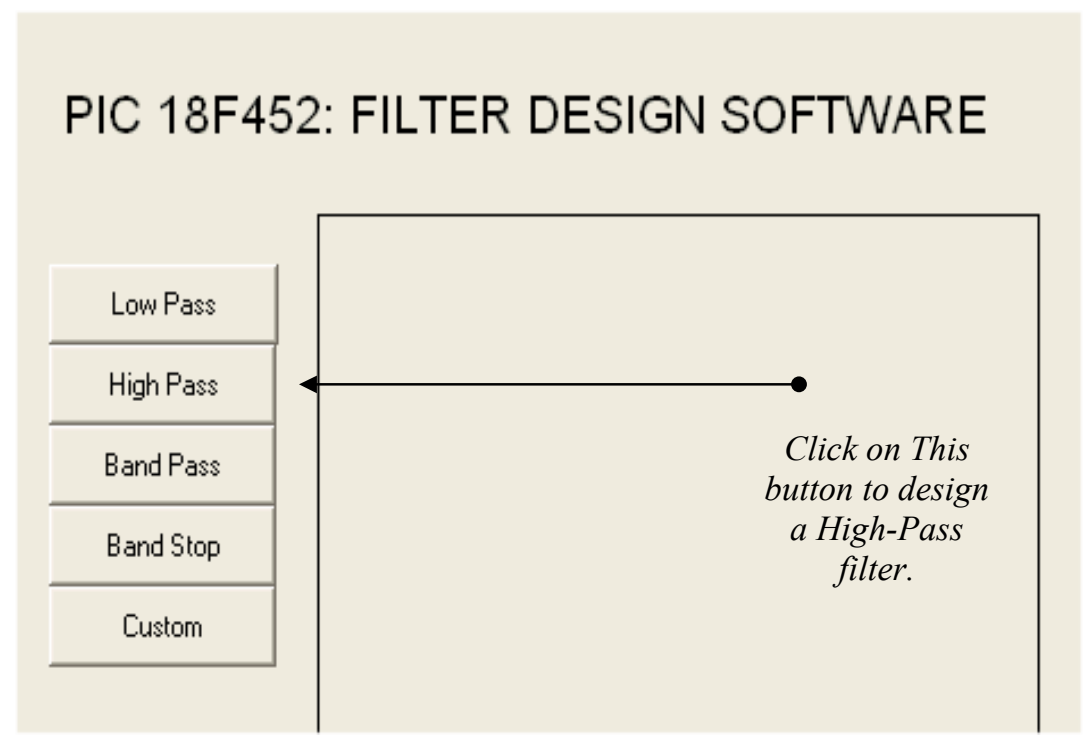

The rest of the design follows exactly the same set of steps as the low pass filter design. 


\section{BAND PASS FILTER DESIGN}

Designing the band-pass filter starts as the previous ones by clicking on the band-pass button in the main window.

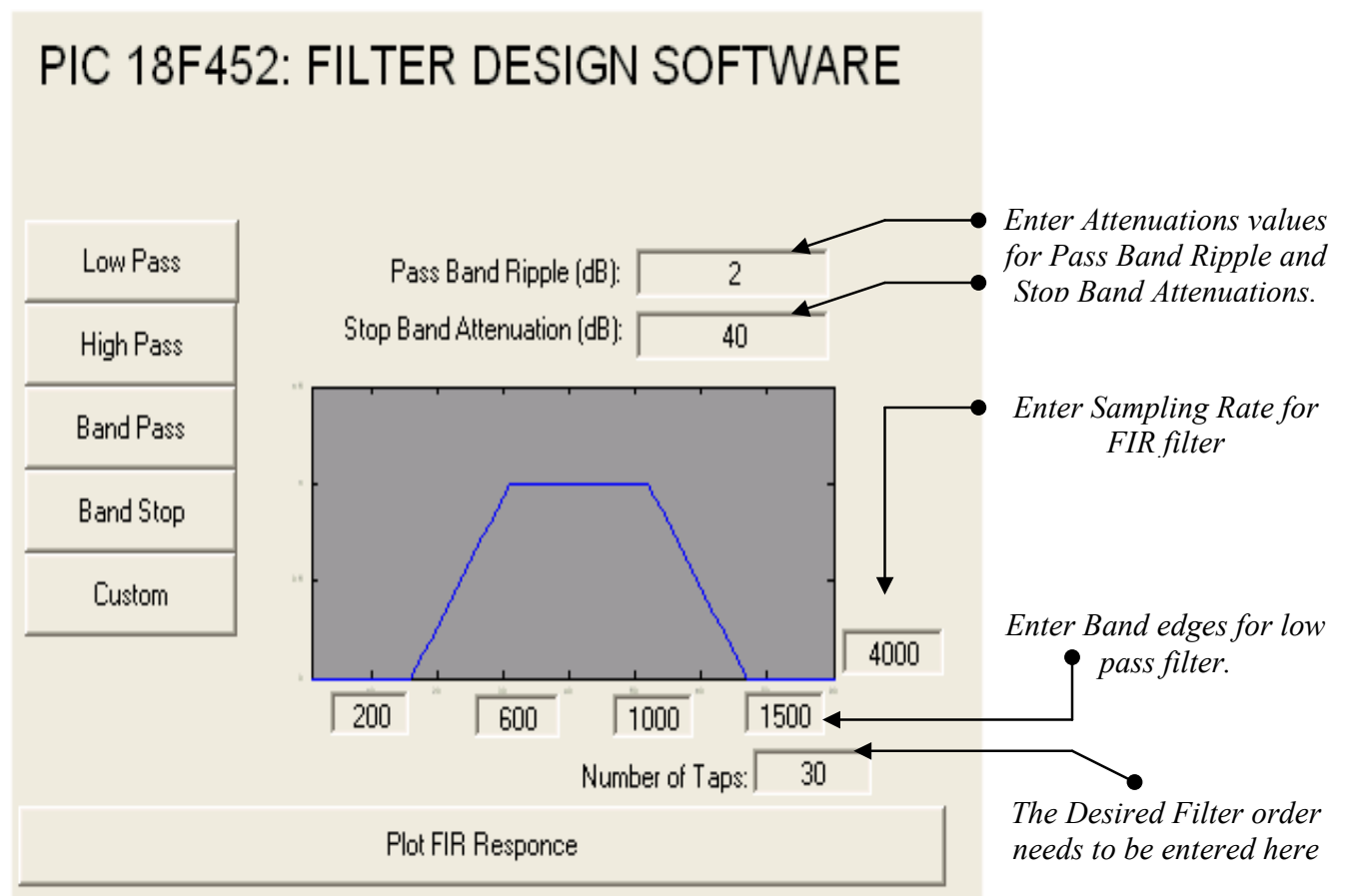

Unlike the LPF and the HPF the optimal number of coefficients are not automatically determined for the FIR band-pass filter configuration. As the Filter order is increased the quality of the filter improves as well.

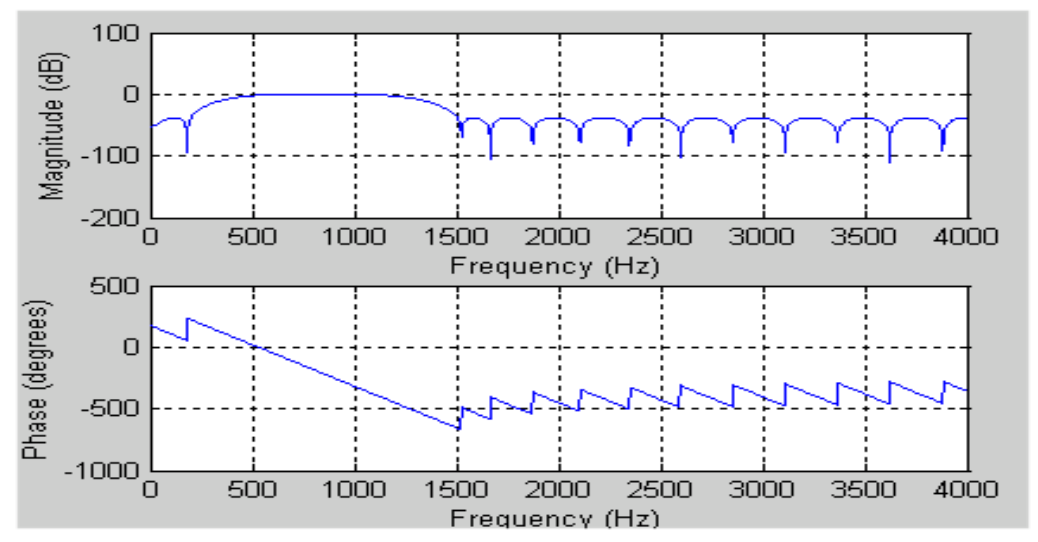




\section{BAND STOP FILTER DESIGN}

Band stop filter is design follows the same set of steps as the Band pass filter. If the filter order is under estimated then the filter response curves indicate the deficiency. Say the user specifies a band stop filter with the given specifications. Notice that only 13 orders are allowed to obtain a $40 \mathrm{db}$ drop in the stop band.

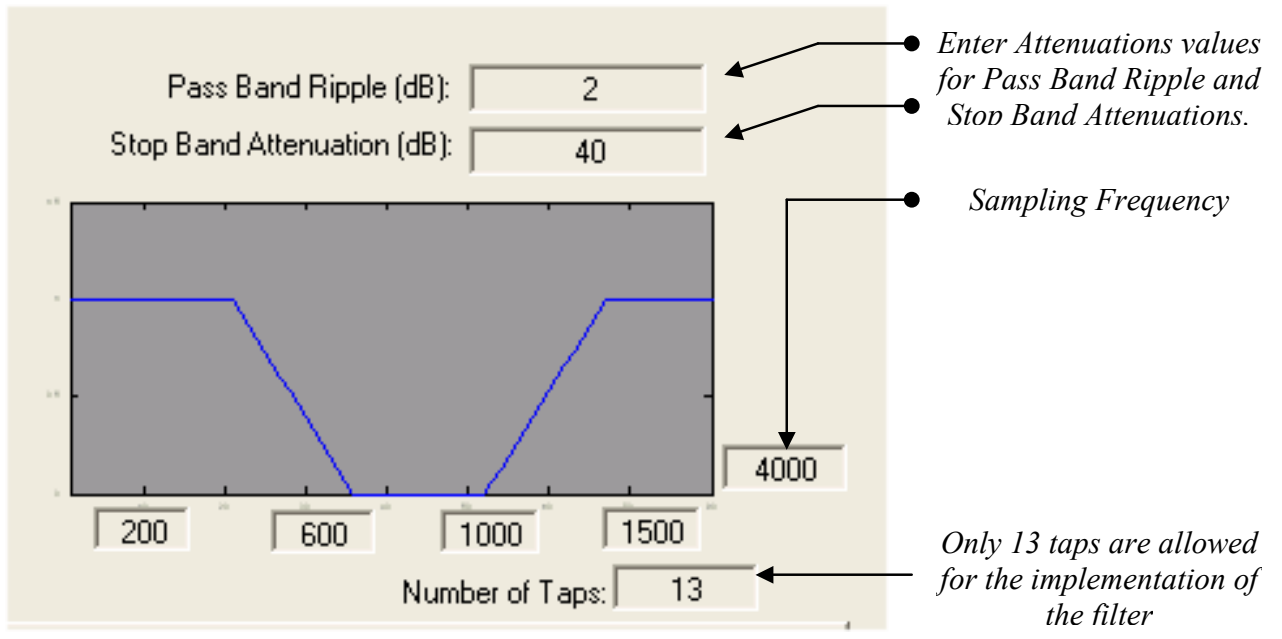

By plotting the frequency and phase curves it is possible to check whether 13 filter taps are enough to attain $40 \mathrm{db}$ in the stop band.

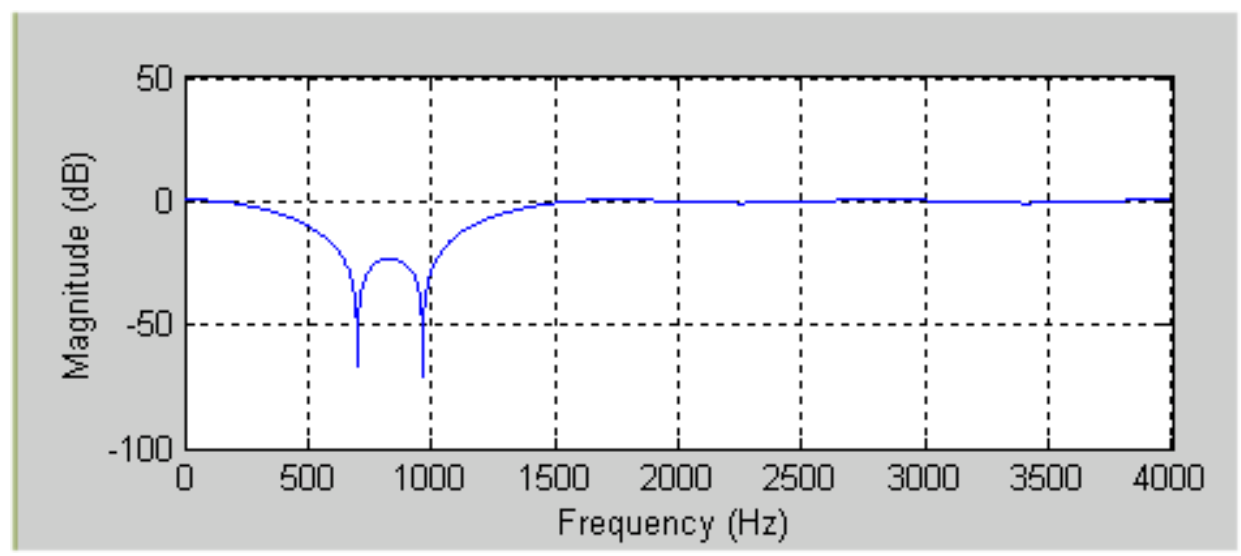


The graph reveals that a 13 -tap filter is not sufficient to attain the desired attenuations. The order must be revised to a higher value, say 40 taps, and the filter curves are plotted again.

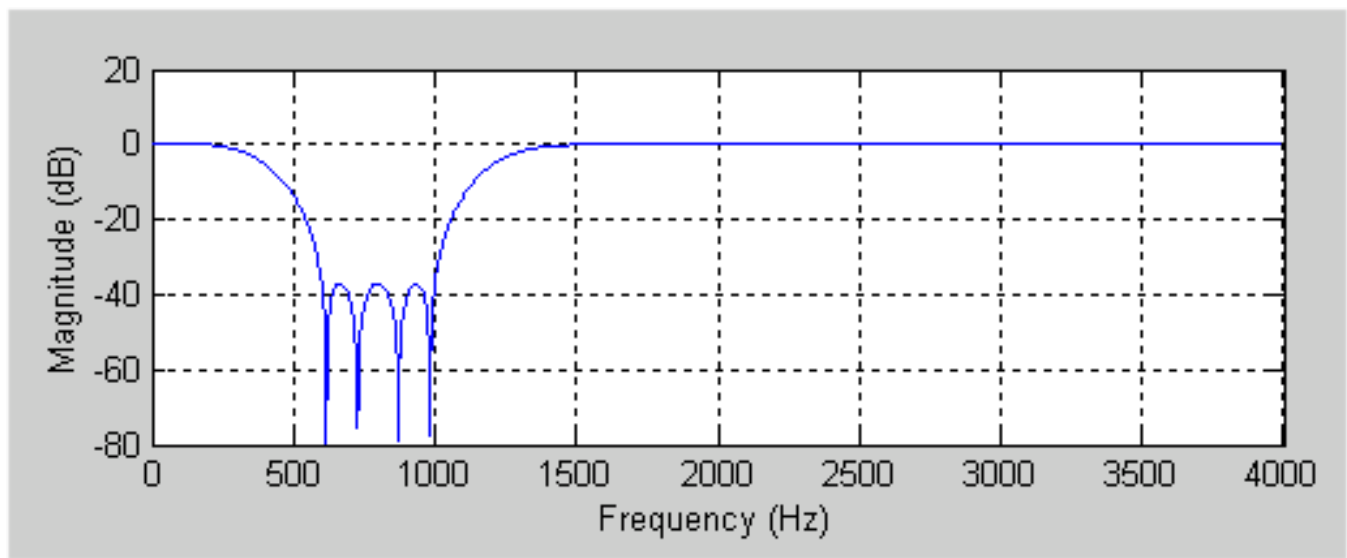

The updated response curve reveals that $40 \mathrm{db}$ drop has been achieved and the code can now be generated in the same manner as before.

\section{CUSTOM FILTER DESIGN}

This is probably the most flexible aspect of FIR filter design because it allows the development of complex filters, which can compensate several different bands at once. The specification of Custom filters is a slightly different than the previous filters. In order to design them the custom button must be selected first in the main window.

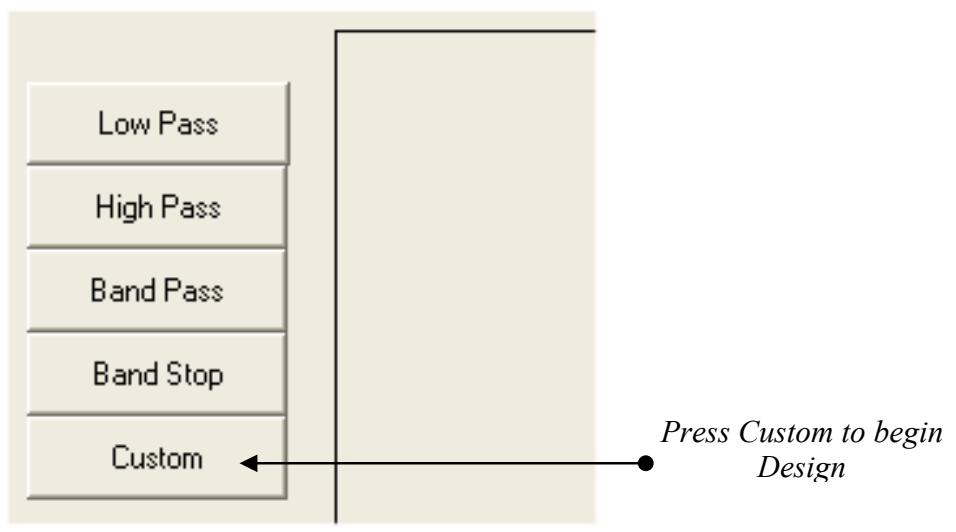


The custom filter design interface is different from the basic filter design interface. In order to create a custom filter four boxes must be filled with the appropriate information. The filter profile, the frequency profile, the band attenuations and the desired filter order.

\section{PIC 18F452: FILTER DESIGN SOFTWARE}

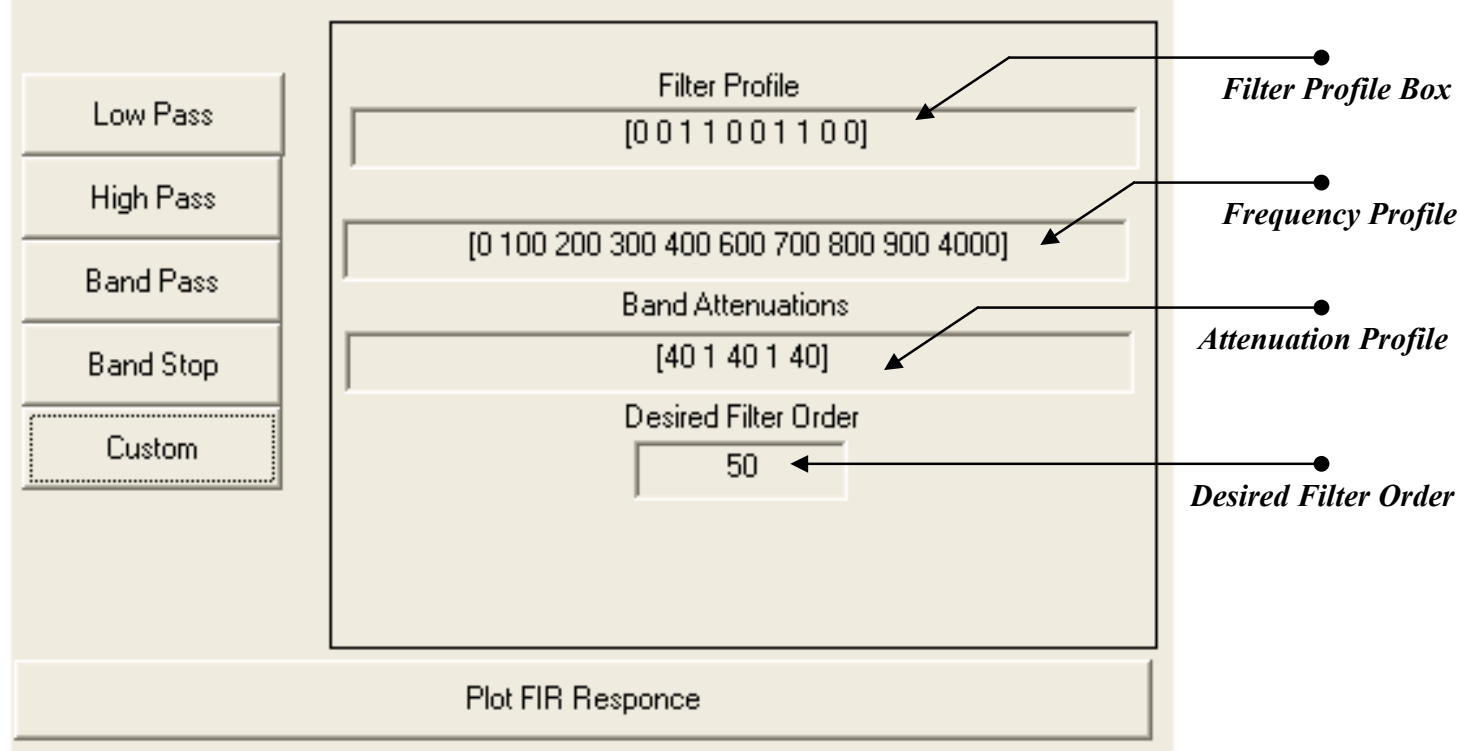

\section{Filter Profile}

Filter profile determines the band edges of the filter. A stop-band is designated by $[0,0]$ and a pass-band is designated by $[1,1]$.

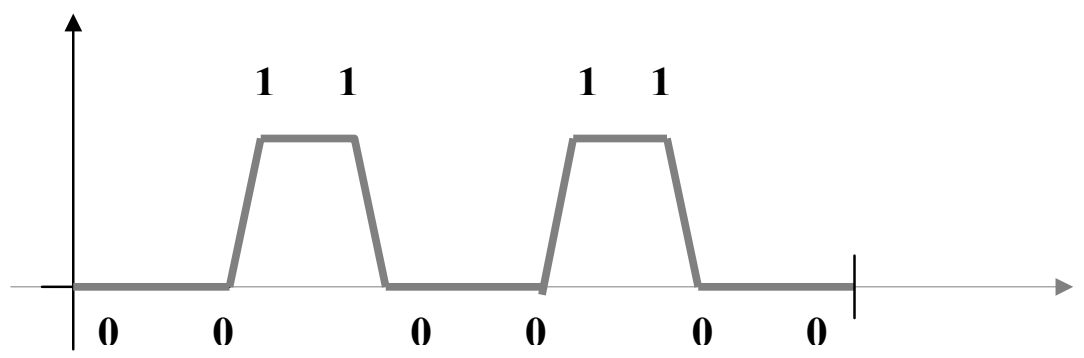

Filter Profile

[0011001100]




\section{Frequency Profile}

The frequency profile is simply the corner frequencies for each band edge and is supplied to the program in the appropriate box.

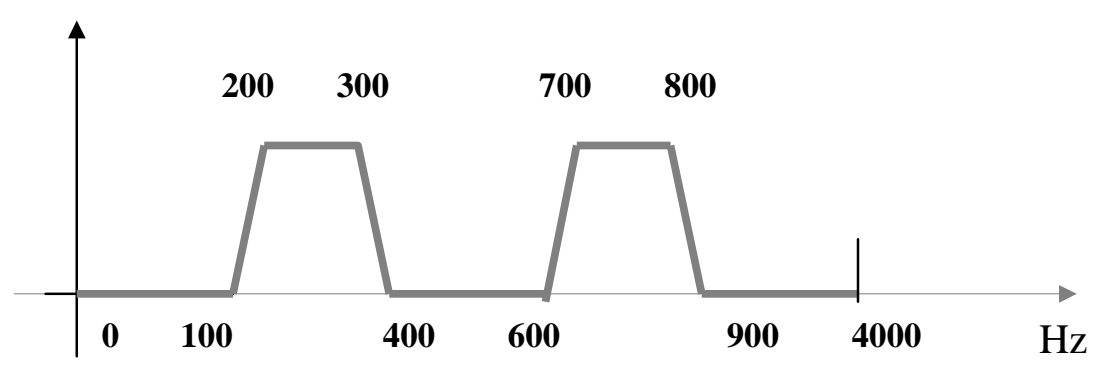

[0 1002003004006007008009004000$]$

\section{Band Attenuation Profile}

Attenuation for each stop-band must be provided as well as the pass band ripple for every pass-band.

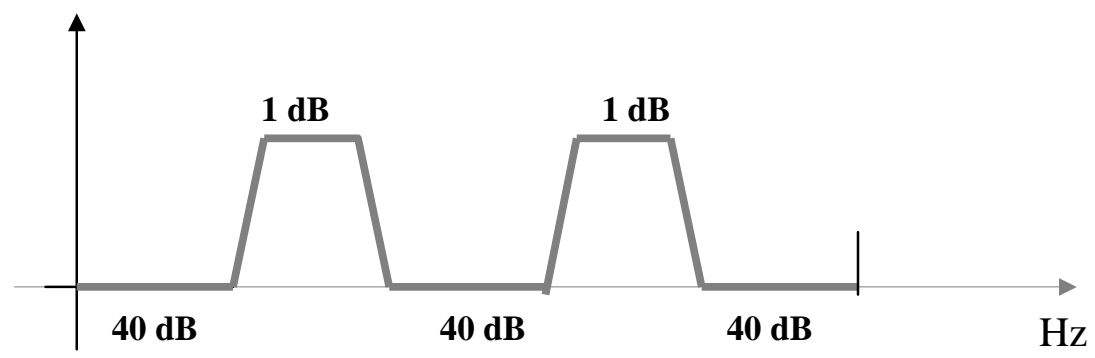

\begin{tabular}{|c|} 
Band Attenuations \\
\hline 40140140$]$
\end{tabular}

\section{Desired Filter Order}

This box is filled with the number of coefficients desired by the designer. As before it is important to check the response curve to make sure the specified attenuations are being correctly met. 
Plotting the frequency response button shows the curves and after increasing the filter order from 50 to 70 the target attenuations are met.

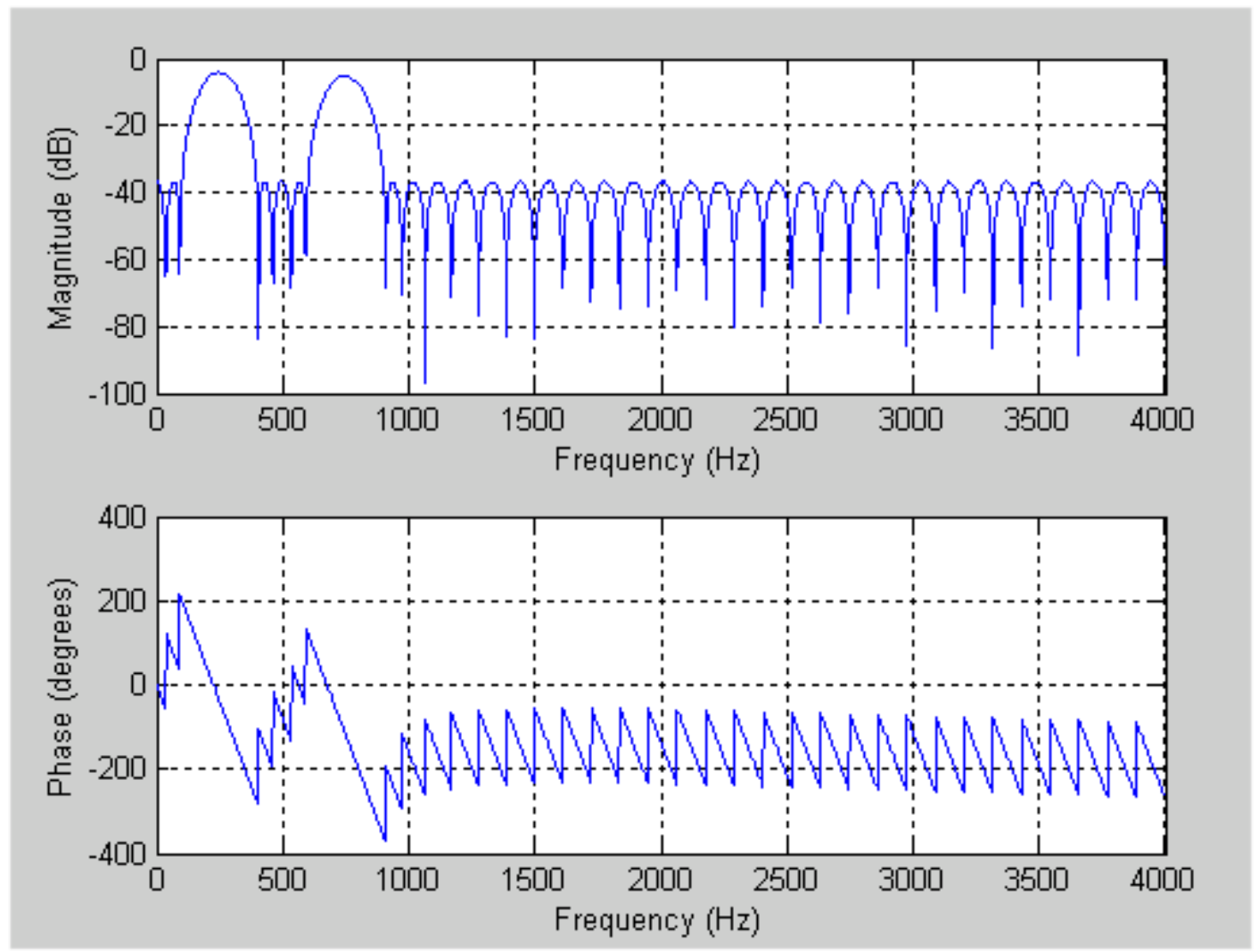

Filter code can be generated as before after selecting an appropriate implementation strategy desired by the user.

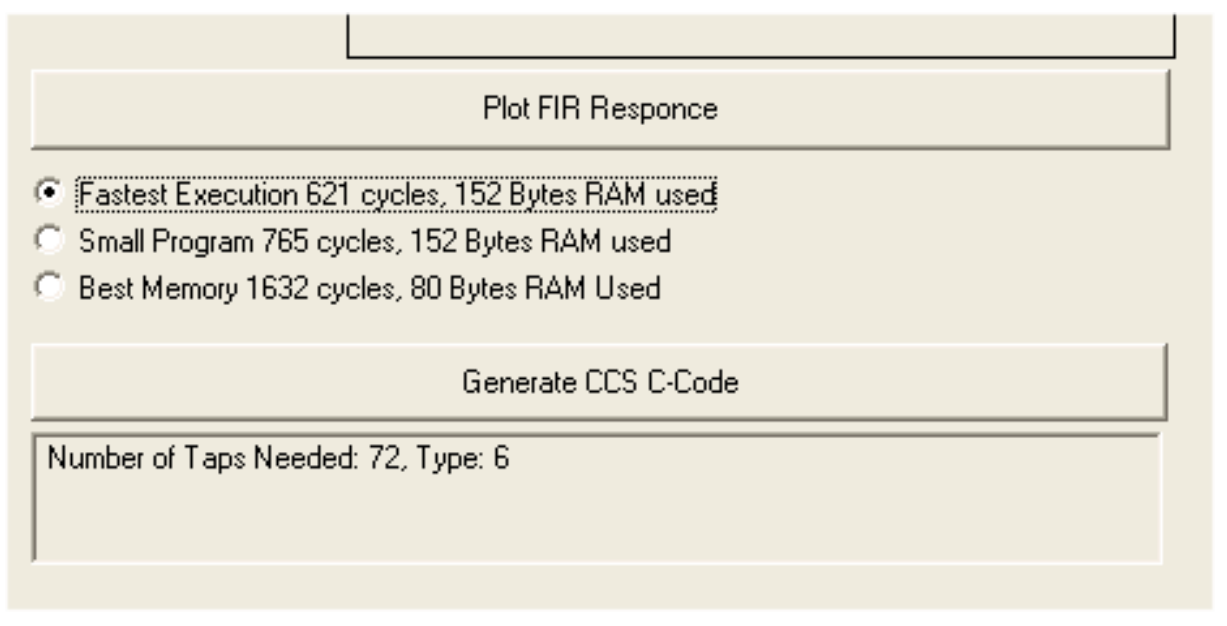




\section{APPENDIX B}

MATLAB CODE FOR FILTER DESIGN SOFTWARE 


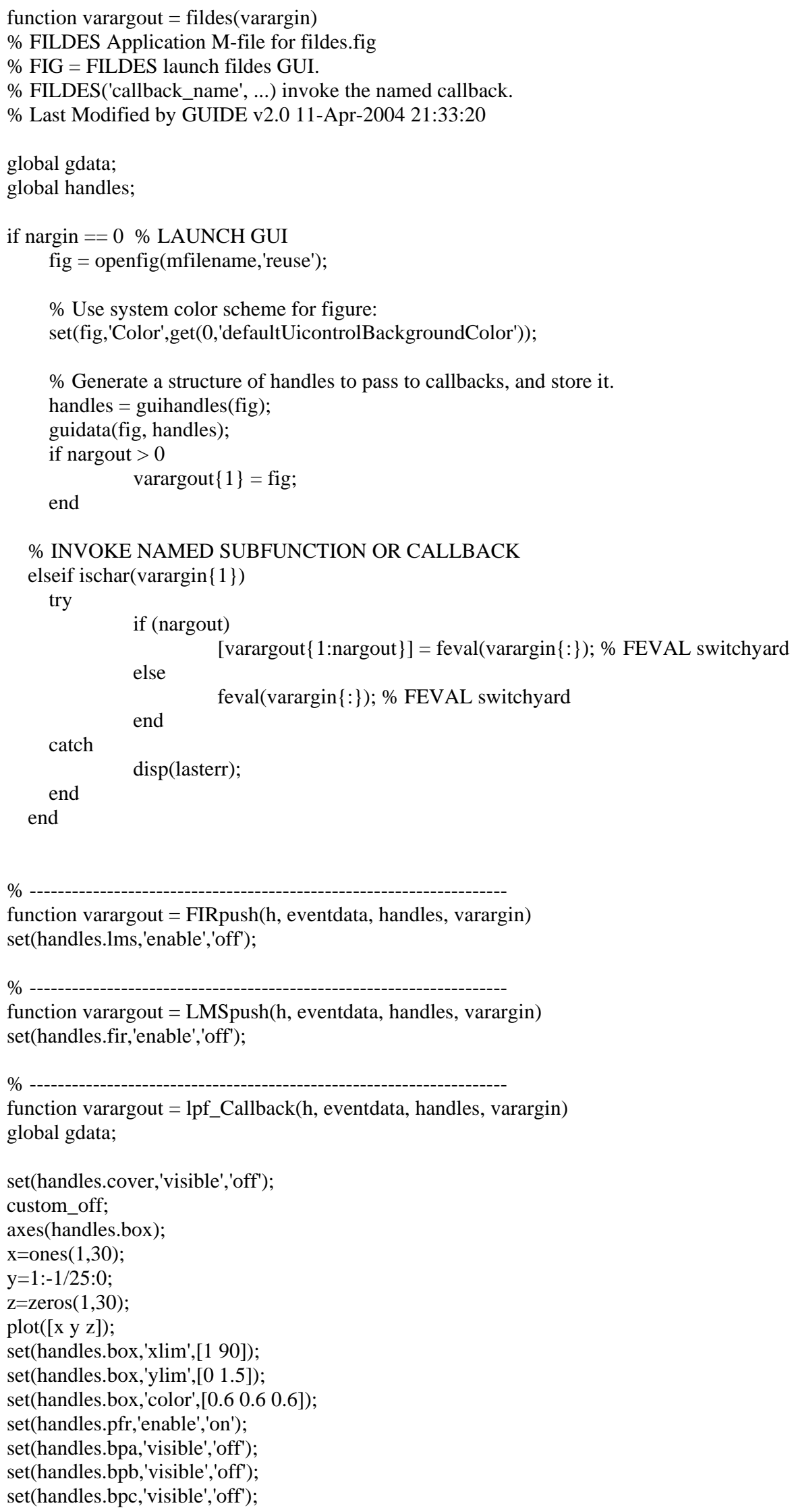


set(handles.bpd,'visible','off'); set(handles.pbco,'visible','on'); set(handles.sbc,'visible','on'); set(handles.tapn,'visible','off'); set(handles.taptext,'visible','off'); gdata.type $=1$;

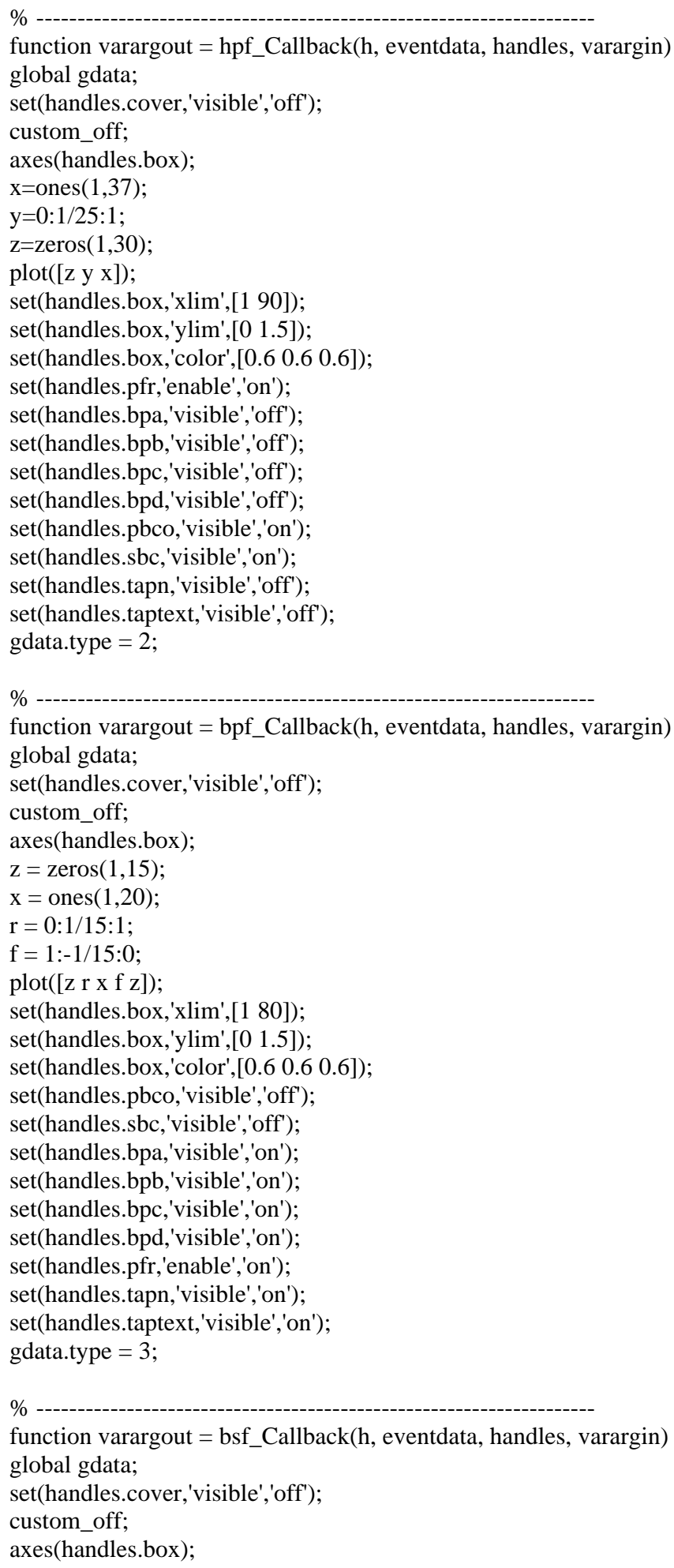




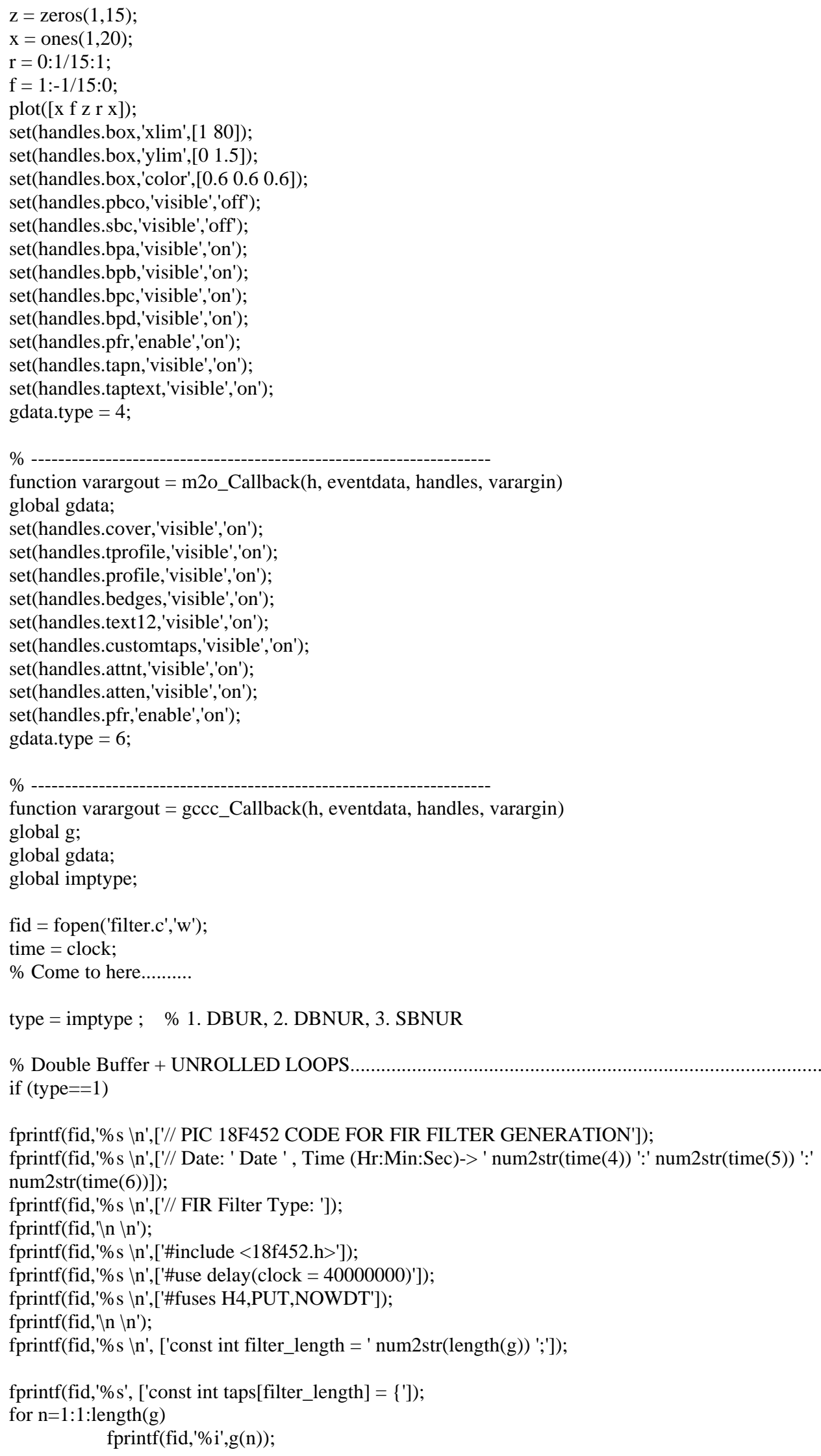




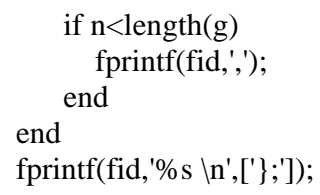


fprintf(fid,'\%s \n',['\#byte TMR2 = 0xfcc ']);

fprintf(fid,'\%s \n',['\#byte T2CON = 0xfca ']);

fprintf(fid,'’n \n');

fprintf(fid,'\%s \n',['// GLOBAL VARIABLES

fprintf(fid,'\%s $\backslash$ ','['int buf0[filter_length] $=\{0\}$;

fprintf(fid,'\%s \n',['int buf1[filter_length] $=\{0\}$;

fprintf(fid,'\%s $\backslash n$ ',['int coef[filter_length] $=\{0\}$;

fprintf(fid, '\%s $\backslash n$ ',['int output_most = 0;

fprintf(fid,'\%s $\backslash n$ ',['int output_middle = 0;

// Store ADC Values

// Store ADC Values ']);

// Store offset Coefficients ']);

fprintf(fid,'\%s \n',['int output_least = 0;

// Most Significant Byte of Output ']);

fprintf(fid,'\%s \n',['int Xn_high_256=0;

fprintf(fid,'\%s $\backslash n$ ',['int Xn_mid_256=0;

fprintf(fid,'\%s \n',['int Xn_low_256=0;

fprintf(fid,'\%s \n',['int Xn_high_128=0;

fprintf(fid,'\%s \n',['int Xn_mid_128=0;

fprintf(fid,'\%s \n',['int Xn_low_128=0;

fprintf(fid,'\%s $\backslash n$ ',['int EOB, MAC_count;

fprintf(fid, '\%s \n',['int n,c, tptr0, tptr1;

// Middle Significant Byte of Output . ']);

// Least Significant Byte of Output ' ]);

// Most Significant Byte of Xn Summation * 255 ']);

// Mid Significant Byte of Xn Summation * 255 ']);

// Least Significant Byte of Xn Summation * 255 ']);

// Most Significant Byte of Xn Summation * 128 ']);

// Mid Significant Byte of Xn Summation * 128 ']);

// Least Significant Byte of Xn Summation * 128 ']);

// Counters for MAC and END of Buffer. ']);

// Temporary Variabes ']);

fprintf(fid,' $\backslash n \backslash n ')$;

fprintf(fid,'\%s \n',['// GLOBAL PROTOTYPES

fprintf(fid,'\%s \n',['void offset_and_buffer_tap_coefficients(void);

fprintf(fid,'\%s \n',['void initialize_pointers(void);']);

fprintf(fid,'`n $\backslash n ')$;

fprintf(fid,'\%s \n',['// INTERRUPT SERVICE ROUTINE'

fprintf(fid,'\%s \n',['\#INT_TIMER2

fprintf(fid,'\%s $\backslash n$ ',['isr() \{'

fprintf(fid, '\%s $\backslash n$ ',['

T2CON = 0x06; // Restart Timer'

fprintf(fid,'\%s $\left.\backslash n^{\prime},["]\right)$;

fprintf(fid, '\%s $\left.\backslash n^{\prime},["]\right)$;

fprintf(fid,'\%s $\backslash n^{\prime},[$ '

FSR0L = tptr0;

fprintf(fid,'\%s $\backslash n$ ',['

FSR1L = tptr1;'

fprintf(fid,'\%s \n',["]);

fprintf(fid,'\%s $\backslash n$ ',['

fprintf(fid, '\%s $\backslash n$ ',['

fprintf(fid, '\%s \n',['

fprintf(fid, '\%s \n',['

fprintf(fid, '\%s $\backslash n$ ',['

fprintf(fid, '\%s $\backslash n$ ',['

fprintf(fid,'\%s $\backslash n$ ',["

fprintf(fid, '\%s $\backslash n$ ',['

fprintf(fid, '\%s \n',['

fprintf(fid, '\%s \n',['

fprintf(fid, '\%s \n',['

fprintf(fid,'\%s $\backslash n$ ',['

fprintf(fid,'\%s \n',['

fprintf(fid, '\%s $\backslash n$ ',['

fprintf(fid, '\%s $\backslash n$ ',["]);

fprintf(fid,'\%s $\backslash n$ ',['

fprintf(fid, '\%s $\backslash n$ ',['

fprintf(fid,'\%s \n',["]);

fprintf(fid, '\%s \n',['

fprintf(fid, '\%s $\backslash n$ ',['

fprintf(fid, '\%s \n',["

fprintf(fid,'\%s $\backslash n$ ',["]);

fprintf(fid, '\%s $\backslash n$ ',['

fprintf(fid,'\%s $\backslash n^{\prime}$, ['

fprintf(fid, '\%s $\backslash n$ ',['

fprintf(fid, '\%s $\backslash n$ ',['

fprintf(fid,'\%s \n',['

fprintf(fid,'\%s \n',['

if $(\mathrm{EOB}==0)\{$

FSROL = \&bufO[0];

FSR1L = \&buf1[0];'

FSR2L = \&coef[0];'

EOB = filter_length;'

\}$^{\prime}$

// Subtract The oldest Xn Value from Total'

\#asm

movf

INDF0, $\mathrm{W}^{\prime}$

subwf Xn_mid_256,F'

clrf WREG'

subwfb Xn_high_256,F'

\#endasm'

]);

]);

] ;

);

']);

]);

]);

']);

);

]);

);

]);

);

);

]);

]);

]);

]);

]);

]);

// Get the latest ADC value;'

WREG = ADRESH;'

] ;

]);

// Restart ADC;

bit_set(ADCON0,2);'

// Add Latest ADC value to Y1(n)' ]);

\#asm']);

$\begin{array}{lcr}\text { movwf } & \text { INDF0']); } & \text { ']); } \\ \text { movwf } & \text { POSTINC1 } & \text { ']); } \\ \text { addwf } & \text { Xn_mid_256,F } & \text { ]); } \\ \text { clrf } & \text { WREG' }\end{array}$




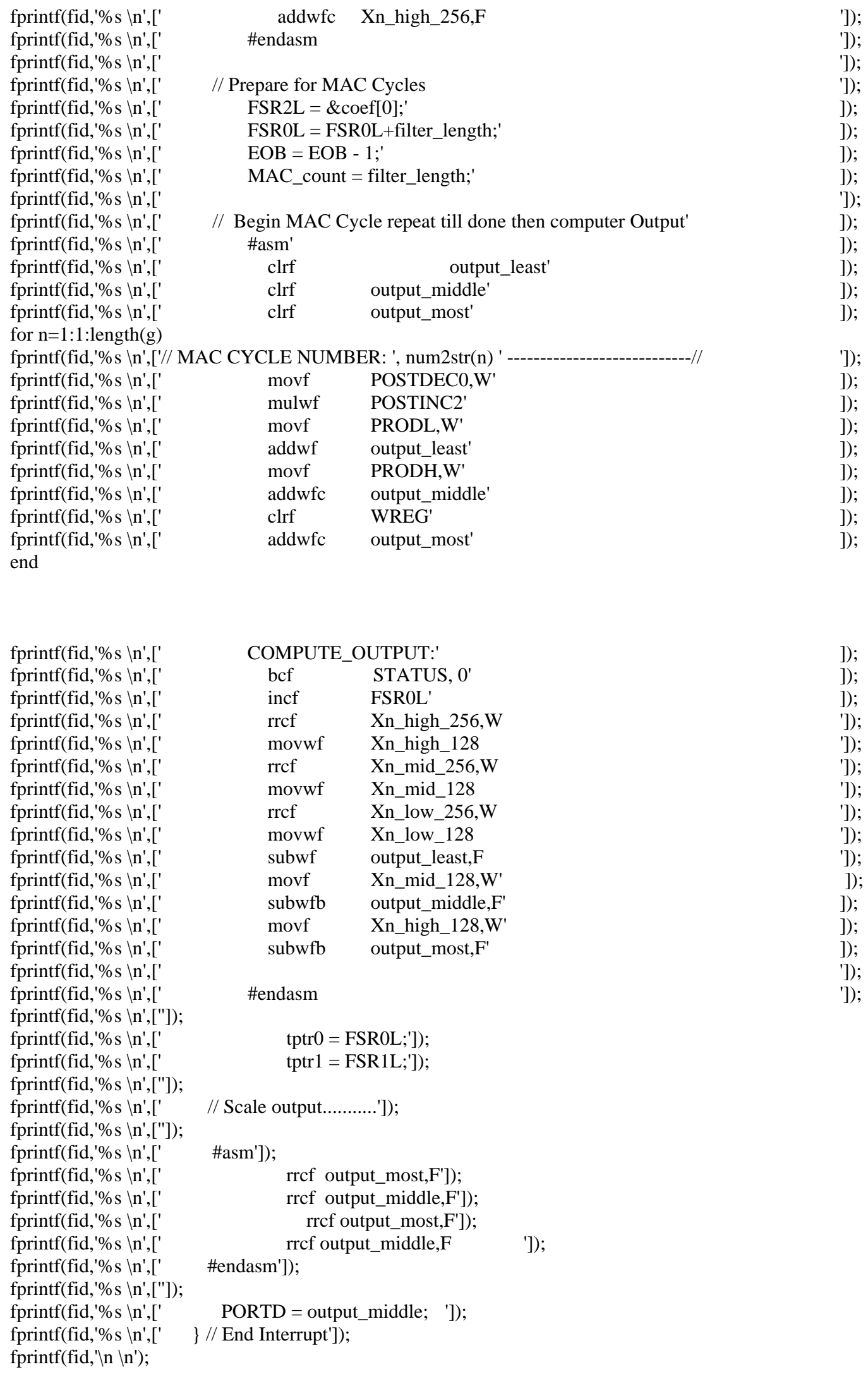


fprintf(fid,'\%s \n',['void main() \{

fprintf(fid, '\%s $\backslash n$ ',['

fprintf(fid, '\%s \n',['

fprintf(fid, '\%s $\backslash n$ ',['

fprintf(fid,'\%s $\backslash n$ ',['

fprintf(fid, '\%s \n',['

fprintf(fid,'\%s $\backslash n$ ',['

fprintf(fid,'\%s \n',['

fprintf(fid, '\%s \n',['

fprintf(fid, '\%s \n',['

fprintf(fid,'\%s \n',['

fprintf(fid, '\%s \n',['

fprintf(fid,'\%s \n',['

fprintf(fid, '\%s \n',['

fprintf(fid, '\%s \n',['

fprintf(fid, '\%s $\backslash n$ ',['

fprintf(fid,'\%s \n',['

fprintf(fid, '\%s \n',['

fprintf(fid, '\%s $\backslash n$ ',['

fprintf(fid,'\%s \n',['

fprintf(fid,'\%s $\backslash n$ ',['

fprintf(fid, $\% s \backslash n ',[$ ['

fprintf(fid, '\%s $\backslash n$ ',['

fprintf(fid,'\%s $\backslash n$ ',['

fprintf(fid, '\%s $\backslash n$ ',['

fprintf(fid, '\%s \n',['

fprintf(fid,'\%s $\backslash n$ ',['

fprintf(fid, '\%s $\backslash n$ ',['

fprintf(fid,'\%s $\backslash n$ ',['

fprintf(fid, '\%s $\backslash n$ ',['

fprintf(fid, '\%s $\backslash n$ ',['

fprintf(fid, '\%s \n',['

fprintf(fid,'\%s $\backslash n$ ',['\}

set_tris_d(0);

// Setup ADC in interrupt mode

setup_adc_ports(ALL_ANALOG);

setup_adc(ADC_CLOCK_DIV_64);

set_adc_channel(0);

// Setup Timer0 in interrupt Mode

$\mathrm{T} 2 \mathrm{CON}=0 \mathrm{x} 06$;

PR2 = 76;

enable_interrupts(INT_TIMER2);

enable_interrupts(GLOBAL); ']);

// FIR filter Code

offset_and_buffer_tap_coefficients(); ']);

// Initialize Pointers

tptr0 = \&buf0[0]; $\quad$ ']);

tptr1 = \&buf1[0]; ']);

FSR2L = \&coef[0]; $\quad$ ']);

EOB = filter_length; ']);

// Start ADC.

bit_set(ADCON0,2); $\quad$ ']);

set_rtcc(65517); ']);

while(1) \{

// Main Application ']);

\} ']);

fprintf(fid,'ไn $\backslash n \backslash n ')$;

fprintf(fid,'\%s \n',['void offset_and_buffer_tap_coefficients(void) \{ ']);

fprintf(fid, '\%s \n',[' int n; $\quad$ ']);

fprintf(fid, $\% s \backslash n$ ',[' for $(\mathrm{n}=0$; $\mathrm{n}<$ filter_length; $\mathrm{n}++)$ \{ $\quad$ ' $]$ );

fprintf(fid, $\%$ s $\backslash n$ ', [' $\quad$ coef[n] = taps[n]+0x80; ' ]);

fprintf(fid,'\%s $\backslash n$ ',[' \} ' ']);

fprintf(fid, $\%$ s $\backslash n$ ',['\}

end

\% DOUBLE BUFFERED: Non UNROLLED LOOPS

if (type $==2$ )

fprintf(fid,'\%s \n',['// PIC 18F452 CODE FOR FIR FILTER GENERATION']);

fprintf(fid,'\%s \n',['// Date: ' Date ' , Time (Hr:Min:Sec)-> ' num2str(time(4)) ':' num2str(time(5)) ':'

num2str(time(6))]);

fprintf(fid,'\%s \n',['// FIR Filter Type: ']);

fprintf(fid,' 'n $\backslash n$ ');

fprintf(fid,'\%s \n',['\#include <18f452.h>']);

fprintf(fid,'\%s \n',['\#use delay(clock = 40000000)']);

fprintf(fid,'\%s $\backslash n$ ',['\#fuses H4,PUT,NOWDT']);

fprintf(fid,' $\backslash n \backslash n ')$;

fprintf(fid,'\%s \n', ['const int filter_length = ' num2str(length(g)) ';']);

fprintf(fid,'\%s', ['const int taps[filter_length] = \{']);

for $\mathrm{n}=1$ :1:length(g) 


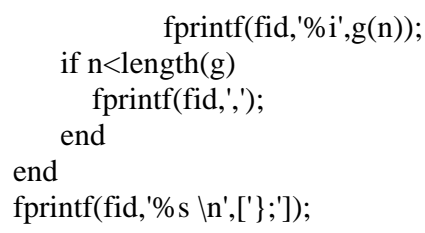

// Hardware File Pointer0 (High) ']);

// Hardware File Pointer0 (Low) ']);

// Hardware File Pointer1 (High) ']);

// Hardware File Pointer1 (Low) ']);

// Hardware File Pointer2 (High) ']);

// Hardware File Pointer2 (Low) ']);

// Read Data Pointed by FSR0 ']);

// Read Data Pointed by FSR1 ']);

// Read Data Pointed by FSR2 ']);

// Add Pointed data to WREG ']);

// Add Pointed data to WREG ']);

// Add Pointed data to WREG ']);

// Pre-increment pointer0 ']);

// Pre-increment pointer1 ']);

// Pre-increment pointer2 ']);

// Post-Incerement Pointer0 ']);

// Post-Decrement Pointer0 ']);

// Post-Increment Pointer1 ']);

// Post-Decrement Pointer1 ']);

// Post-Increment Pointer2 ']);

// Post-Decrement Pointer2 ']);

']);

']);

']); 
fprintf(fid,'\%s $\backslash n$ ',['\#byte PR2 = 0xfcb

fprintf(fid,'\%s $\backslash n$ ',['\#byte TMR2 = 0xfcc

']);

fprintf(fid,'\%s \n',['\#byte T2CON = 0xfca

']);

fprintf(fid,' $\backslash n \backslash n ')$;

fprintf(fid,'\%s $\backslash n$,'['// GLOBAL VARIABLES

fprintf(fid, '\%s $\backslash n$ ',['int buf0[filter_length] $=\{0\}$;

fprintf(fid,'\%s $\backslash$ ','['int buf1[filter_length] $=\{0\}$;

fprintf(fid,'\%s $\backslash$ ','['int coef[filter_length] $=\{0\}$;

fprintf(fid,'\%s $\backslash n$ ',['int output_most = 0;

fprintf(fid,'\%s \n',['int output_middle = 0;

fprintf(fid,'\%s $\backslash n$ ',['int output_least = 0;

fprintf(fid,'\%s \n',['int Xn_high_256=0;

fprintf(fid,'\%s \n',['int Xn_mid_256=0;

fprintf(fid,'\%s $\backslash n$ ',['int Xn_low_256=0;

fprintf(fid,'\%s $\backslash n$ ',['int Xn_high_128=0;

fprintf(fid,'\%s $\backslash n$ ',['int Xn_mid_128=0;

fprintf(fid,'\%s $\backslash n$ ',['int Xn_low_128=0;

fprintf(fid,'\%s $\backslash n$ ',['int EOB, MAC_count;

fprintf(fid,'\%s \n',['int n,c, tptr0, tptr1;

// Store ADC Values

// Store ADC Values

// Store offset Coefficients

// Most Significant Byte of 0

// Middle Significant Byte of Output ']);

// Least Significant Byte of Output ']);

// Most Significant Byte of Xn Summation * 255 ']);

// Mid Significant Byte of Xn Summation * 255 ']);

// Least Significant Byte of Xn Summation * 255 ']);

// Most Significant Byte of Xn Summation * 128 ']);

// Mid Significant Byte of Xn Summation * 128 ']);

// Least Significant Byte of Xn Summation * 128 ']);

// Counters for MAC and END of Buffer. ']);

// Temporary Variabes ']);

fprintf(fid,'’n $\backslash n$ ');

fprintf(fid,'\%s \n',['// GLOBAL PROTOTYPES

fprintf(fid,'\%s \n',['void offset_and_buffer_tap_coefficients(void); $\quad$ ']);

fprintf(fid,'\%s \n',['void initialize_pointers(void);

fprintf(fid,'ไn $\backslash n$ ');

fprintf(fid,'\%s \n',['// INTERRUPT SERVICE ROUTINE

fprintf(fid,'\%s \n',['\#INT_TIMER2 ']);

fprintf(fid,'\%s $\backslash n$ ',['isr() \{ ']);

fprintf(fid, $\% \mathrm{~s} \backslash \mathrm{n}$ ',[' T2CON = 0x06; // Restart Timer $\quad$ ']);

fprintf(fid,'\%s $\backslash n$ ',['

fprintf(fid,'\%s $\backslash n$ ',['

fprintf(fid, '\%s \n',['

fprintf(fid,'\%s $\backslash n^{\prime},['$

fprintf(fid, '\%s \n',['

fprintf(fid, $\%$ s $\backslash n$ ', [' if $(\mathrm{EOB}==0)\{$

fprintf(fid,'\%s \n',['

FSR0L = tptr0;

FSR1L = tptr1;

fprintf(fid, '\%s \n',['

fprintf(fid,'\%s \n',['

fprintf(fid, '\%s \n',['

fprintf(fid,'\%s $\backslash n$ ',['

fprintf(fid, '\%s \n',['

fprintf(fid, '\%s $\backslash n$ ',['

fprintf(fid, '\%s $\backslash n$ ',['

fprintf(fid, '\%s \n',['

fprintf(fid, '\%s $\backslash n$ ',['

fprintf(fid, '\%s $\backslash n$ ',['

fprintf(fid,'\%s \n',['

fprintf(fid, '\%s \n',['

fprintf(fid, '\%s \n',['

fprintf(fid, '\%s \n',['

fprintf(fid, '\%s \n',['

fprintf(fid, '\%s \n',['

fprintf(fid,'\%s \n',['

fprintf(fid, '\%s \n',['

fprintf(fid, '\%s $\backslash n$ ',['

fprintf(fid, '\%s \n',['

fprintf(fid, '\%s \n',['

fprintf(fid, '\%s $\backslash n$ ',['

fprintf(fid,'\%s \n',['

fprintf(fid, '\%s \n',['

fprintf(fid, '\%s $\backslash n$ ',['

fprintf(fid,'\%s \n',['

fprintf(fid,'\%s \n',['

\}

FSR0L = \&buf0[0];

FSR1L = \&buf1[0];

FSR2L = \&coef[0]; $\quad$ ']);

EOB = filter_length; ']);

']);

// Subtract The oldest Xn Value from Total ']);

']);

movf INDF0,W ']);

subwf Xn_mid_256,F ']);

clrf WREG ']);

$\begin{array}{lll}\text { subwfb Xn_high_256,F } & \text { ']); } \\ & \end{array}$

WREG = ADRESH;

bit set(ADCONO,2);

']);

/l Add Latest ADC value to $\mathrm{Y} 1(\mathrm{n})$ - ']);

\#asm

movwf INDF0 ']);

movwf POSTINC1 ']);

addwf Xn_mid_256,F ']);

clrf WREG ']);

addwfc Xn_high_256,F ']); 


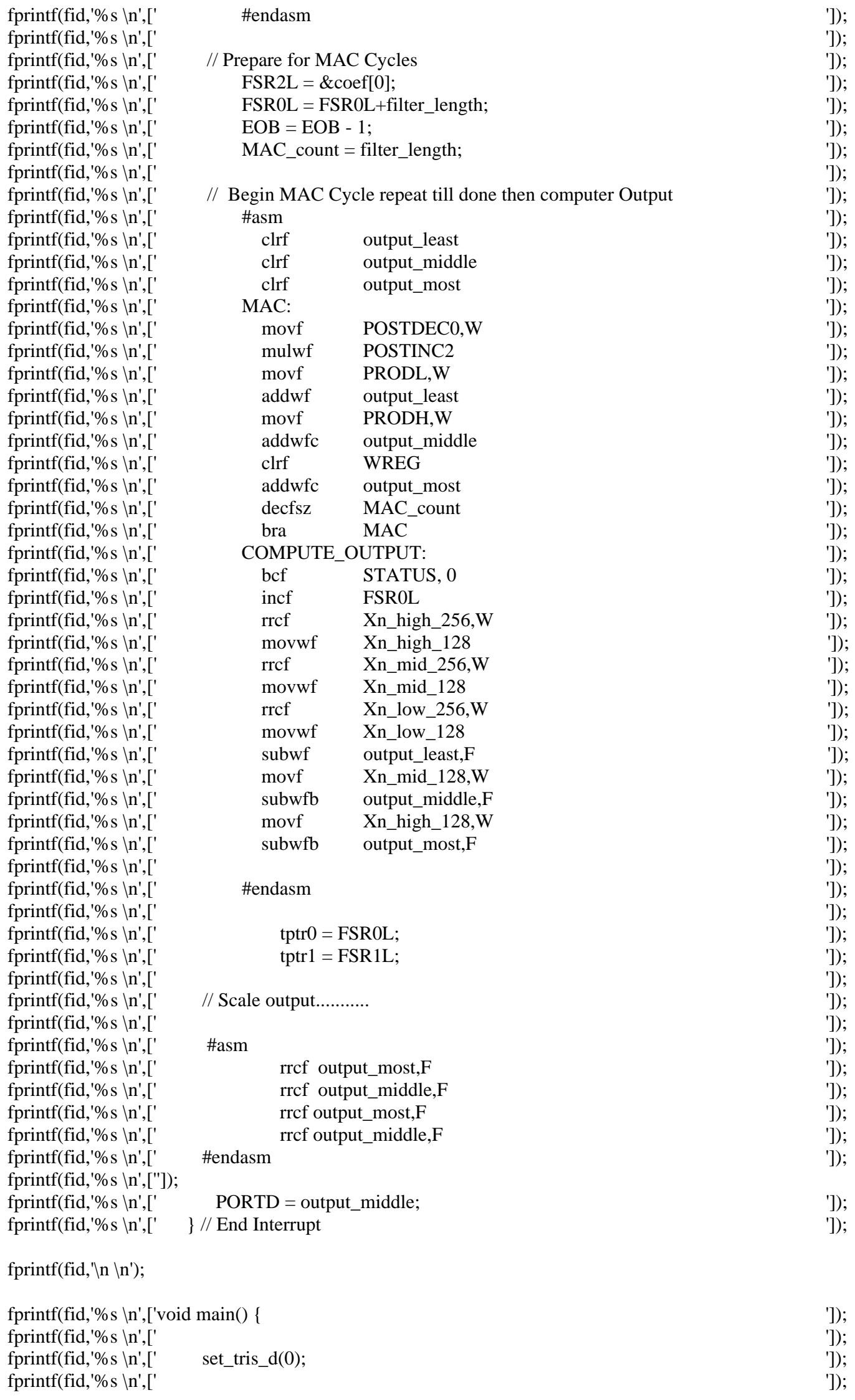




\begin{tabular}{|c|c|c|}
\hline fprintf(fid,'\%s \n',[' & // Setup ADC in interrupt mode & ]); \\
\hline fprintf(fid,'\%s \n',[' & setup_adc_ports(ALL_ANALOG); & ]); \\
\hline fprintf(fid,'\%s \n',[' & setup_adc(ADC_CLOCK_DIV_64); & ]); \\
\hline fprintf(fid,'\%s \n',[' & set_adc_channel(0); & ]); \\
\hline fprintf(fid,'\%s $\backslash n^{\prime},\left[{ }^{\prime}\right.$ & & ]); \\
\hline fprintf(fid,'\%s \n',[' & // Setup Timer0 in interrupt Mode & ]); \\
\hline fprintf(fid,'\%s \n',[' & T2CON = 0x06; & ]); \\
\hline fprintf(fid,'\%s \n',[' & $\mathrm{PR} 2=76$ & ]); \\
\hline fprintf(fid,'\%s \n',[' & enable_interrupts(INT_TIMER2); & ]); \\
\hline fprintf(fid,'\%s \n',[' & enable_interrupts(GLOBAL); & ]); \\
\hline fprintf(fid,'\%s \n',[' & & ]); \\
\hline fprintf(fid,'\%s \n',[' & // FIR filter Code & ]); \\
\hline fprintf(fid,'\%s \n',[' & offset_and_buffer_tap_coefficients(); & ']); \\
\hline fprintf(fid,'\%s \n',[' & & ']); \\
\hline fprintf(fid,'\%s \n',[' & // Initialize Pointers & ']); \\
\hline fprintf(fid,'\%s $\backslash n$ ',[' & $\operatorname{tptr} 0=$ \&buf0 $[0]$ & ']); \\
\hline fprintf(fid,'\%s $\backslash n$ ',[' & $\operatorname{tptr} 1=$ \&buf1[0]; & ']); \\
\hline fprintf(fid,'\%s \n',[' & FSR2L = \&coef $[0]$ & ']); \\
\hline fprintf(fid,'\%s \n',[' & EOB = filter_length; & ']); \\
\hline fprintf(fid,'\%s \n',[' & & ']); \\
\hline fprintf(fid,'\%s \n',[' & // Start ADC. & ']); \\
\hline fprintf(fid,'\%s \n',[' & bit_set(ADCON0,2); & ']); \\
\hline fprintf(fid,'\%s \n',[' & set_rtcc(65517); & ']); \\
\hline fprintf(fid,'\%s \n',[' & & ']); \\
\hline fprintf(fid,'\%s $\backslash n^{\prime},\left[{ }^{\prime}\right.$ & // Main Loop & ']); \\
\hline fprintf(fid,'\%s \n',[' & while(1) \{ & ']); \\
\hline fprintf(fid,'\%s \n',[' & // Main Application & ']); \\
\hline fprintf(fid,'\%s \n',[' & \} & ']); \\
\hline fprintf(fid,'\%s $\backslash n$ ',['\} & & ']); \\
\hline fprintf(fid,' $\backslash n \backslash n \backslash n ')$; & & \\
\hline fprintf(fid,'\%s \n',['vo & id offset_and_buffer_tap_coefficients(void) \{ & \\
\hline fprintf(fid,'\%s \n',[' & int $n$ & \\
\hline fprintf(fid,'\%s \n',[' & for $(\mathrm{n}=0 ; \mathrm{n}<$ filter_length; $\mathrm{n}++)\{$ & ']); \\
\hline fprintf(fid,'\%s \n',[' & $\operatorname{coef}[\mathrm{n}]=\operatorname{taps}[\mathrm{n}]+0 \times 80$ & \\
\hline fprintf(fid,'\%s \n',[' & 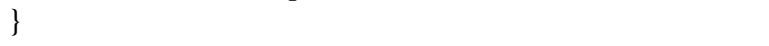 & ' ]); \\
\hline fprintf(fid,'\%s \n',['\} & & \\
\hline
\end{tabular}

\% SINGLE BUFFERD: Non UNROLLED LOOPS

if (type $==3$ )

fprintf(fid,'\%s \n',['// PIC 18F452 CODE FOR FIR FILTER GENERATION

']);

fprintf(fid,'\%s \n',['// Date: ' Date ' , Time (Hr:Min:Sec)-> ' num2str(time(4)) ':' num2str(time(5)) ':'

num2str(time(6))

fprintf(fid, '\%s \n',['// FIR Filter Type:

fprintf(fid,'\n\n');

fprintf(fid,'\%s \n',['\#include <18f452.h>

fprintf(fid,'\%s \n',['\#use delay(clock = 40000000)

fprintf(fid,'\%s \n',['\#fuses H4,PUT,NOWDT

fprintf(fid,'’n $\backslash n$ ');

fprintf(fid,'\%s \n', ['const int filter_length = ' num2str(length(g)) '; $\quad$ ']);

fprintf(fid,'\%s', ['const int taps[filter_length] $=\{$

for $\mathrm{n}=1: 1:$ length(g)

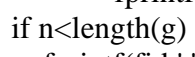

end

fprintf(fid,'\%s $\backslash n^{\prime},['\}$;

']);

fprintf(fid,'\%s \n',['// PIC 18F452 Register MAP.................................................................................// ']);

fprintf(fid,'\%s \n',[' 
fprintf(fid,'\%s $\backslash n$ ',['// ACCUMULATOR ADDRESS

fprintf(fid,'\%s $\backslash n$ ',['\#byte WREG = 0xFE8

fprintf(fid,'\%s $\backslash n$ ',['\#byte PRODL =0xff3

fprintf(fid,'\%s \n',['\#byte PRODH =0xff4

fprintf(fid,'\%s $\backslash n$ ',['\#byte ADRESL = 0xfc3

fprintf(fid,'\%s \n',['\#byte ADRESH = 0xfc4

fprintf(fid,'\%s $\backslash n$ ',['\#byte STATUS = 0xfd8

fprintf(fid,'\%s \n',['

fprintf(fid,'\%s $\backslash n$ ',['// DC CONTROL REGISTERS

fprintf(fid,'\%s $\backslash n$ ',['\#byte ADCON0 = 0xfc2

fprintf(fid,'\%s \n',['\#byte ADCON1 = 0xfc1

fprintf(fid,'\%s \n',['\#byte ADRESL = 0xfc3

fprintf(fid,'\%s \n',['\#byte ADRESH = 0xfc4

fprintf(fid,'\%s \n',['

fprintf(fid, '\%s \n',['

fprintf(fid,'\%s $\backslash n$ ',['// DIGITAL IO PORT ADDRESSES

fprintf(fid,'\%s $\backslash n$ ',['\#byte PORTA = 0xf80

fprintf(fid,'\%s $\backslash n$ ',['\#byte PORTB $=0 x f 81$

fprintf(fid,'\%s $\backslash n$ ', ['\#byte PORTC = 0xf82

fprintf(fid,'\%s $\backslash n$ ',['\#byte PORTD = 0xf83

fprintf(fid,'\%s $\backslash n$ ',['\#byte PORTE = 0xf84

fprintf(fid,'\%s \n',['\#byte LATA = 0xf89

// Port A Address

// Port B Address

// Port C Address

// Port D Address

// Port E Address

fprintf(fid, '\%s $\backslash n$ ' [“

// Set Driection for PORTA

fprintf(fid, '\%s $\backslash n$ ',['

fprintf(fid,'\%s $\backslash n$ ',['// INDIRECT ADDRESSING

fprintf(fid,'\%s \n',['\#byte FSR0H = 0xfeA

fprintf(fid,'\%s $\backslash n$ ',['\#byte FSR0L = 0xfe9

fprintf(fid,'\%s $\backslash n$ ',['\#byte FSR1H = 0xfe2

fprintf(fid,'\%s $\backslash n$ ',['\#byte FSR1L = 0xfe1

fprintf(fid,'\%s $\backslash n$ ',['\#byte FSR2H = 0xfda

fprintf(fid,'\%s \n',['\#byte FSR2L = 0xfd9

fprintf(fid,'\%s \n',['\#byte INDF0 = 0xfef

fprintf(fid,'\%s $\backslash n$ ',['\#byte INDF1 = 0xfe7

fprintf(fid,'\%s $\backslash n$ ',['\#byte INDF2 = 0xfdf

fprintf(fid,'\%s \n',['\#byte PLUSW0 = 0xfeb

fprintf(fid,'\%s $\backslash n$ ',['\#byte PLUSW1 = 0xfe3

fprintf(fid,'\%s $\backslash n$ ',['\#byte PLUSW2 = 0xfdb

fprintf(fid, '\%s \n',['\#byte PREINC0 = 0xfec

fprintf(fid, '\%s \n',['\#byte PREINC1 = 0xfe4

fprintf(fid,'\%s \n',['\#byte PREINC2 = 0xfdc

fprintf(fid,'\%s \n',['\#byte POSTINC0 = 0xfee

fprintf(fid,'\%s $\backslash n$ ',['\#byte POSTDEC0 = 0xfed

fprintf(fid,'\%s \n',['\#byte POSTINC1 = 0xfe6

fprintf(fid,'\%s $\backslash n$ ',['\#byte POSTDEC1 = 0xfe5

fprintf(fid,'\%s $\backslash n$ ',['\#byte POSTINC2 = 0xfde

// Hardware File Pointer0 (High)

// Hardware File Pointer0 (Low)

// Hardware File Pointer1 (High)

// Hardware File Pointer1 (Low)

// Hardware File Pointer2 (High)

// Hardware File Pointer2 (Low)

// Read Data Pointed by FSR0

// Read Data Pointed by FSR1

// Read Data Pointed by FSR2

// Add Pointed data to WREG

// Add Pointed data to WREG

// Add Pointed data to WREG

// Pre-increment pointer0

// Pre-increment pointer1

// Pre-increment pointer2

// Post-Incerement Pointer0

// Post-Decrement Pointer0

// Post-Increment Pointer1

// Post-Decrement Pointer1

// Post-Increment Pointer2

fprintf(fid,'\%s \n',['\#byte POSTDEC2 = 0xfdd

// Post-Decrement Pointer2

fprintf(fid, '\%s $\backslash n$ ',['

fprintf(fid, '\%s $\backslash n$ ',['

fprintf(fid,'\%s $\backslash n$,'['// TIMER REGISTERS

fprintf(fid, '\%s $\backslash n$ ',['\#byte PR2 = 0xfcb

fprintf(fid, '\%s $\backslash n$ ',['\#byte TMR2 = 0xfcc

fprintf(fid, '\%s $\backslash n$ ',['\#byte T2CON = 0xfca

fprintf(fid,' $\backslash n \backslash n ')$;

fprintf(fid,'\%s $\backslash n$ ', ['int buf[filter_length] $=\{0\}$;

fprintf(fid,'\%s $\backslash n$ ', ['int coef[filter_length] $=\{0\}$; $\quad$ // Store offset Coefficients

fprintf(fid,'\%s \n', ['int output_most =0; // Most Significant Byte of Output .....

fprintf(fid, $\%$ s \n', ['int output_middle = 0; // Middle Significant Byte of Output .............

fprintf(fid,'\%s \n', ['int output_least = 0; // Least Significant Byte of Output ............. I

fprintf(fid,'\%s \n', ['int Xn_high_256=0; // Most Significant Byte of Xn Summation * 255.....।

fprintf(fid,'\%s \n', ['int Xn_mid_256=0; // Mid Significant Byte of Xn Summation * 255...... II

fprintf(fid,'\%s \n', ['int Xn_low_256=0; // Least Significant Byte of Xn Summation * 255....।

fprintf(fid,'\%s \n', ['int Xn_high_128=0; // Most Significant Byte of Xn Summation * 128.....।

']);

']);

']);

']);

']);

']);

']);

']);

']);

']);

']);

']);

']);

']);

']);

']);

']);

']);

']);

']);

']);

']);

']);

']);

']);

']);

']);

']);

']);

']);

']);

']);

I);

']);

']);

]);

']);

']);

']);

']);

']);

']);

']);

']);

']);

']);

']);

']);

']);

']);

']);

']);

']);

']);

']);

']);

']);

']);

']);

']); 
fprintf(fid,'\%s \n', ['int Xn_mid_128=0; // Mid Significant Byte of Xn Summation * 128....... \ ']); fprintf(fid,'\%s \n', ['int Xn_low_128=0; ／/ Least Significant Byte of Xn Summation * 128....। I]); fprintf(fid,'’n $\backslash n$ ');

fprintf(fid,'\%s \n\n', [' // General Globals

fprintf(fid,'\%s $\backslash n \backslash n$ ', ['int b,EOB,BOB,x,tptr, out, mac_count; ']);

fprintf(fid,'\%s $\backslash n \backslash n$ ', [' // FIR Filter Prototypes

fprintf(fid,'\%s $\backslash n \backslash n$ ', ['void offset_and_buffer_tap_coefficients(void);
fprintf(fid,' $\backslash n \backslash n ')$;

fprintf(fid,'\%s \n', ['\#INT_TIMER2 ']);

fprintf(fid,'\%s \n', ['void t2_isr() \{

fprintf(fid, $\%$ s $\backslash n$ ', [' $\quad$ T2CON = 0x06; // Restart Timer ']);

fprintf(fid, '\%s \n', [' $\quad$ ADCON0 = 0x8d; $\quad$ // Start ADC Conversion ']);

fprintf(fid,'\%s \n', [' $\quad$ while(bit_test(ADCON0,2)); // Wait for Conversion to Complete ']);

fprintf(fid,'\%s $\backslash n$ ', [' ']);

fprintf(fid,'\%s $\backslash n$ ', [' b = ADRESH; $\quad$ // Read ADC Value ']);

$\begin{array}{ll}\text { fprintf(fid,'\%s } \backslash n \text { ', ["]); } & \text { FSR0L = tptr; }\end{array}$

fprintf(fid, '\%s \n', [' // Subtract the oldest ADC value in buffer from total ']);

fprintf(fid, $\% s \backslash n$ ', ['

fprintf(fid,'\%s $\backslash n$ ', [' \#asm ']);

fprintf(fid,'\%s $\backslash n$ ', [' movf INDF0,W $\quad$ ']);

fprintf(fid,'\%s $\backslash n$ ', [' Subwf Xn_mid_256,F ']

fprintf(fid,'\%s $\backslash n$ ', [' clrf WREG $\quad$ ']);

fprintf(fid,'\%s \n', [' subwfb Xn_high_256,F ']);

fprintf(fid,'\%s $\backslash n$ ', ['

fprintf(fid,'\%s $\backslash n$ ', [' // Add the latest ADC value to the buffer ']);

fprintf(fid, '\%s \n', [' ']);

fprintf(fid,'\%s $\backslash$ n', [' movf EOB,0 // Move to W Register ']);

fprintf(fid,'\%s $\backslash n$ ', [' cpfseq FSR0L // Check if ptr is at EOB ']);

fprintf(fid,'\%s $\backslash n$ ', [' bra neq ']);

fprintf(fid,'\%s \n', [' movff b,INDF0 // ptr has reached EOB: insert value ']);

fprintf(fid,'\%s $\backslash n$ ', [' movff BOB,FSR0L // Reset pointer to begining of Buffer']);

fprintf(fid,'\%s $\backslash n$ ', ['

fprintf(fid,'\%s $\backslash n$ ', ['

fprintf(fid,'\%s $\backslash n$ ', ['

fprintf(fid,'\%s $\backslash n$ ', ['

fprintf(fid,'\%s \n', ["]);

fprintf(fid,'\%s $\backslash n$ ', ['

fprintf(fid, '\%s $\backslash n$ ', ['

fprintf(fid,'\%s $\backslash n$ ', ['

fprintf(fid,'\%s $\backslash n$ ', ['

fprintf(fid,'\%s \n', ['

fprintf(fid,'\%s $\backslash n$ ', ['

fprintf(fid,'\%s $\backslash n$ ', ['

fprintf(fid, '\%s $\backslash n$ ', ['

fprintf(fid,'\%s $\backslash n$ ', ['

fprintf(fid,'\%s $\backslash n$ ', ['

fprintf(fid,'\%s $\backslash n$ ', [' bra end']);

neq:

']); end:

movff b,POSTDEC0 // Put data in Buffer and advance ptr']);

fprintf(fid, '\%s $\backslash n$ ', ['

fprintf(fid,'\%s $\backslash n$ ', ['

fprintf(fid,'\%s $\backslash n$ ', ['

fprintf(fid,'\%s $\backslash n$ ', ['

fprintf(fid,'\%s $\backslash n$ ', ['

fprintf(fid,'\%s $\backslash n$ ', ['

fprintf(fid, '\%s $\backslash n$ ', ['

fprintf(fid,'\%s $\backslash n$ ', ["]);

fprintf(fid,'\%s $\backslash n$ ', ['

fprintf(fid,'\%s $\backslash n$ ', ["]);

fprintf(fid,'\%s $\backslash n$ ', ['

fprintf(fid,'\%s $\backslash n$ ', ['

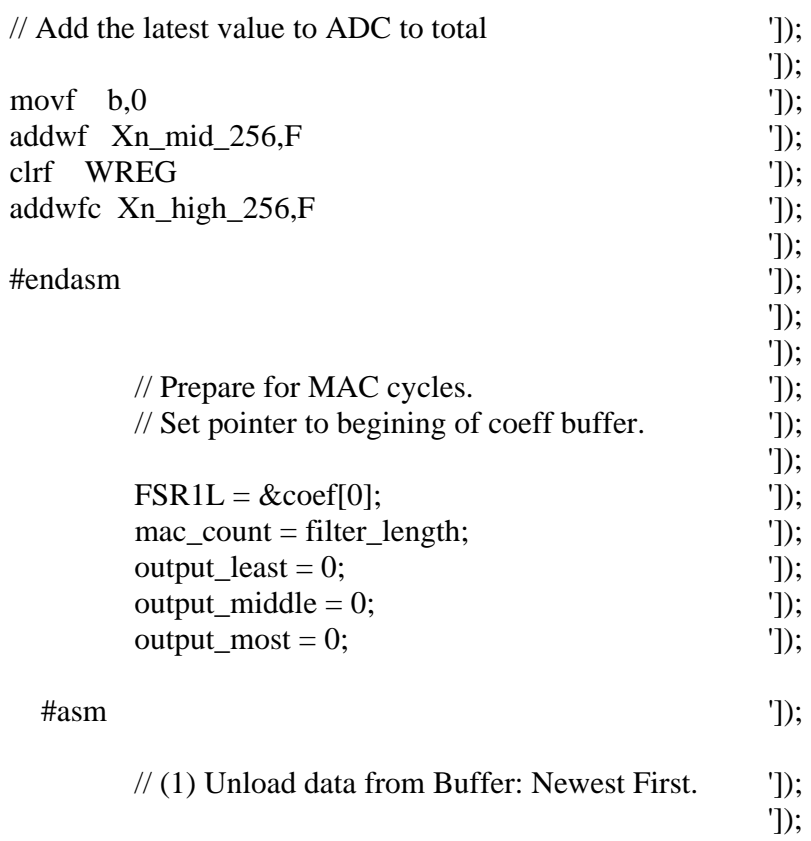


fprintf(fid, '\%s $\backslash n$ ', [' fprintf(fid, '\%s \n', [' fprintf(fid, '\%s \n', [' fprintf(fid, '\%s \n', [' fprintf(fid, '\%s $\backslash n$ ', [' fprintf(fid, '\%s \n', [' fprintf(fid,'\%s $\backslash n$ ', [' fprintf(fid,'\%s \n', [' fprintf(fid,'\%s $\backslash n$ ', [' fprintf(fid, '\%s $\backslash n$ ', [' fprintf(fid, '\%s $\backslash n$ ', [' fprintf(fid,'\%s \n', [' fprintf(fid, '\%s \n', [' fprintf(fid,'\%s \n', [' fprintf(fid, '\%s \n', [' fprintf(fid,'\%s \n', [' fprintf(fid,'\%s \n', [' fprintf(fid,'\%s \n', [' fprintf(fid, '\%s $\backslash n$ ', [' fprintf(fid,'\%s \n', [' fprintf(fid,'\%s $\backslash n$ ', [' fprintf(fid,'\%s $\backslash n$ ', [' fprintf(fid, '\%s $\backslash n$ ', [' fprintf(fid,'\%s \n', [' fprintf(fid, '\%s $\backslash n$ ', [' fprintf(fid, '\%s \n', [' fprintf(fid,'\%s $\backslash n$ ', [' fprintf(fid, '\%s $\backslash n^{\prime}$, [' fprintf(fid, '\%s $\backslash n$ ', [' fprintf(fid,'\%s \n', [' fprintf(fid, '\%s $\backslash n$ ', [' fprintf(fid,'\%s \n', [' fprintf(fid,'\%s \n', [' fprintf(fid, '\%s \n', [' fprintf(fid,'\%s \n', [' fprintf(fid, '\%s \n', [' fprintf(fid, '\%s \n', [' fprintf(fid,'\%s \n', [' fprintf(fid, '\%s \n', [' fprintf(fid,'\%s \n', [' fprintf(fid,'\%s $\backslash n$ ', [' fprintf(fid,'\%s \n', [' fprintf(fid,'\%s \n', [' fprintf(fid,'\%s \n', ["]); fprintf(fid,'\%s \n', [' fprintf(fid,'\%s \n', [' fprintf(fid, '\%s \n', [' fprintf(fid, '\%s $\backslash n$ ', [' fprintf(fid, '\%s \n', [' fprintf(fid,'\%s \n', [' fprintf(fid, '\%s $\backslash n$ ', [' fprintf(fid,'\%s \n', [' fprintf(fid,'\%s $\backslash n$ ', [' fprintf(fid, '\%s $\backslash n$ ', [' fprintf(fid,'`n \n'); fprintf(fid,'\%s $\backslash n$ ', [' fprintf(fid, '\%s $\backslash n$ ', [' fprintf(fid, '\%s \n', [' fprintf(fid,'\%s $\backslash n$ ', [' fprintf(fid, '\%s $\backslash n$ ', [' fprintf(fid, '\%s \n', [' fprintf(fid,'\%s $\backslash n$ ', [' mac:

movf BOB,0 // Move to W Register ']);

cpfseq FSR0L // Check if ptr is at BOB ']);

bra aneq ']);

movff EOB,FSR0L // Pointer is at BOB.. Warp Pointer to EOB ']);

movff INDF0,out // Extract Data ']);

bra aend ']);

aneq: $\quad$ ']);

movff PREINC0,out // Extract Data from Buffer ']);

aend: $\quad$ ']);

// (2) Perform MAC cycle.

movf out,W

mulwf POSTINC1 ']);

movf PRODL,W ']);

addwf output_least ']);

movf PRODH,W ']);

addwfc output_middle ']);

clrf WREG ']);

addwfc output_most ']);

decfsz mac_count ']);

bra mac ']);

// (3) Compute output.

']);

bcf STATUS,0']);

rrcf Xn_high_256,W ']);

movwf Xn_high_128 ']);

rrcf Xn_mid_256,W ']);

movwf Xn_mid_128 ']);

rrcf Xn_low_256,W ']);

movwf Xn_low_128 ']);

subwf output_least,F ']);

movf Xn_mid_128,W ']);

subwfb output_middle,F ']);

movf Xn_high_128,W ']);

subwfb output_most,F ']);

(')

tptr = FSR0L; $\quad$ ']);

// Scale output............ ']);

\#asm ']);

bcf STATUS,0 ']);

rrcf output_most,F ']);

rrcf output_middle,F ']);

rrcf output_most,F ']);

\#endasm ']);

PORTD = output_middle; $\quad$ ']);

\} (1);

void main() \{ ']);

set_tris_d(0); ']);

$\mathrm{x}=0$; $\quad$ ']);

T2CON = 0x06; 


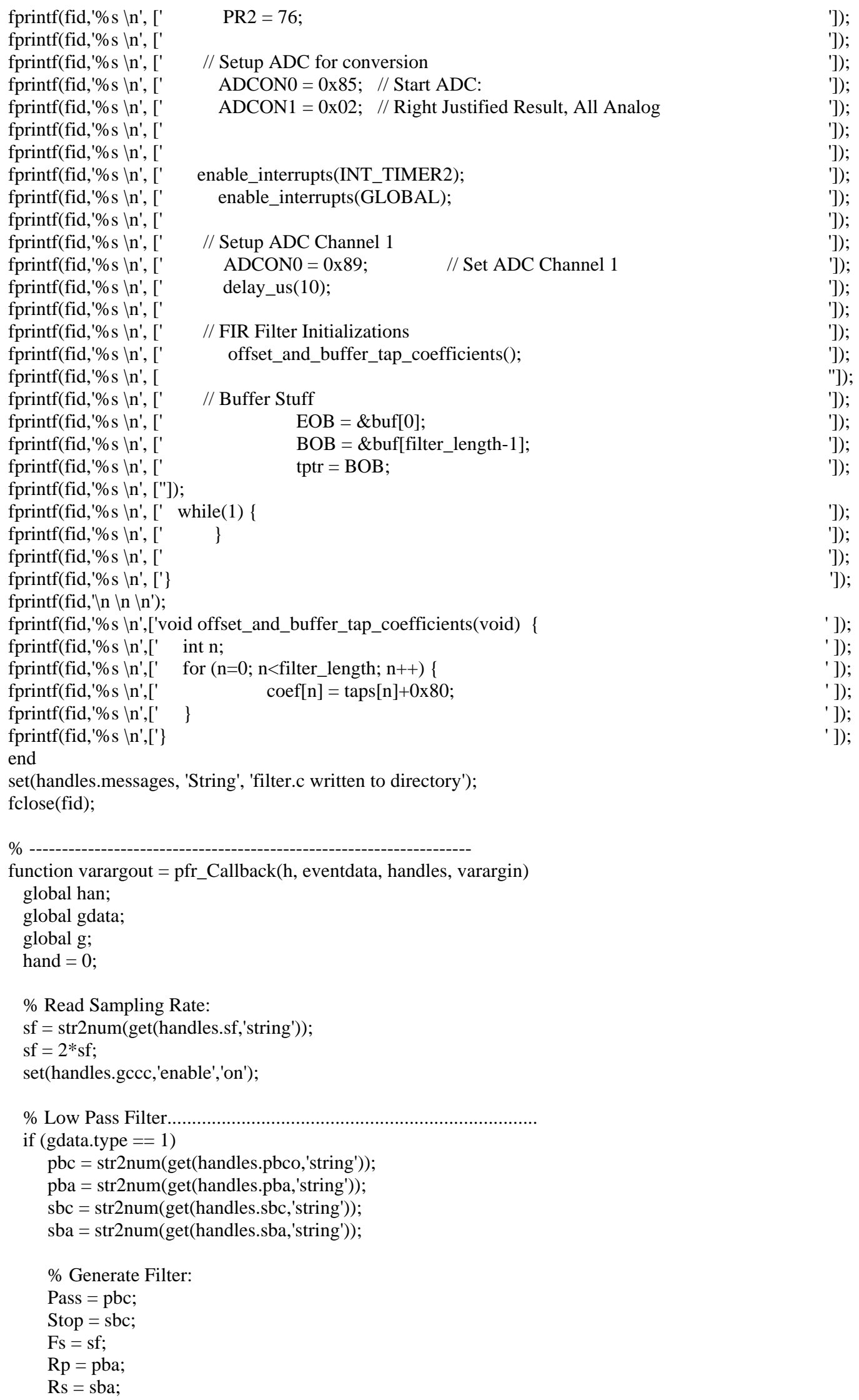




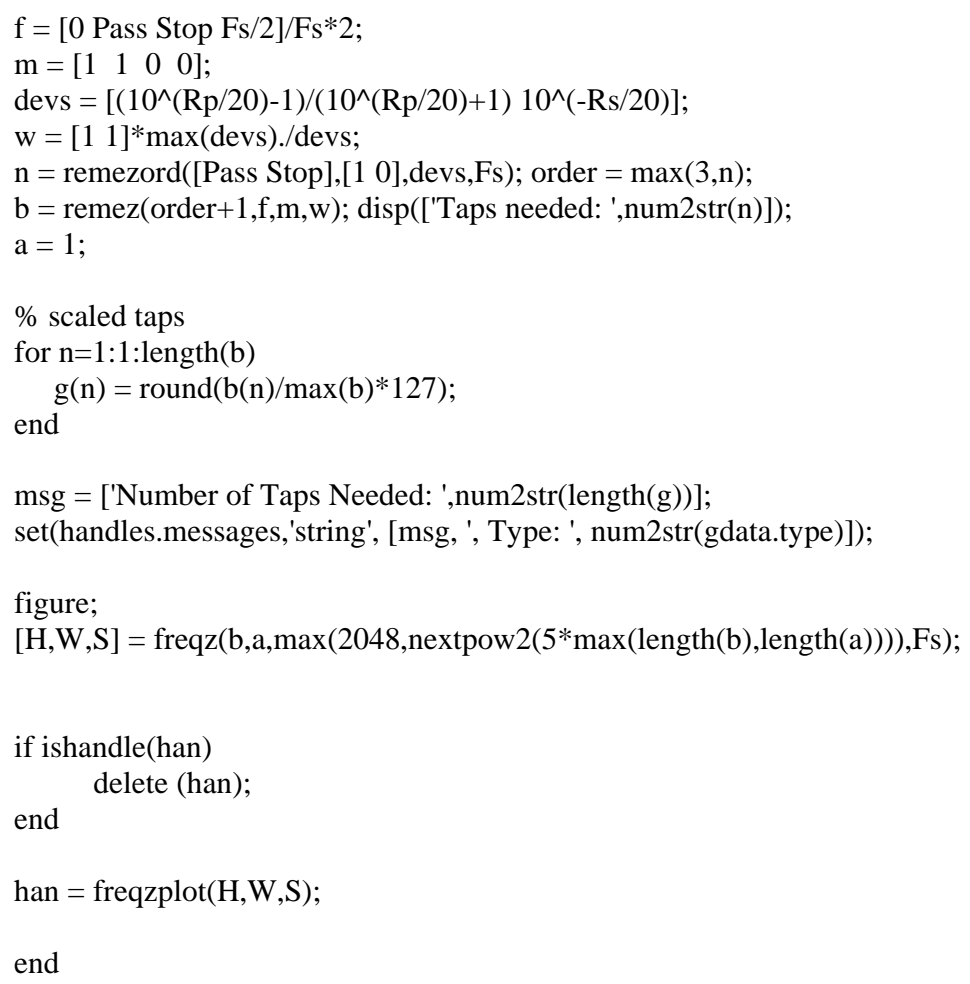




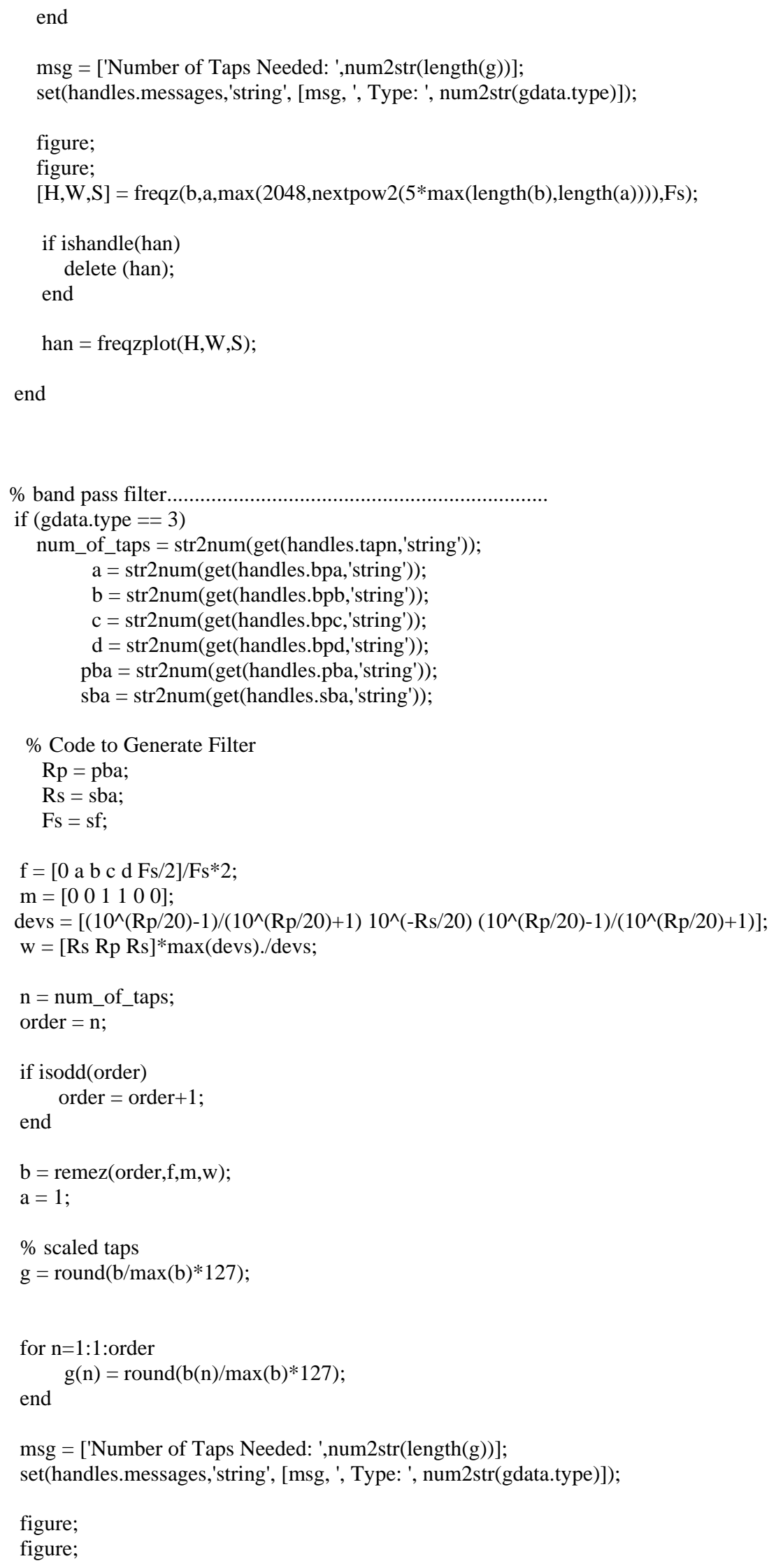


[H,W,S] = freqz(b,a,max(2048,nextpow2(5*max(length(b),length(a)))),Fs);

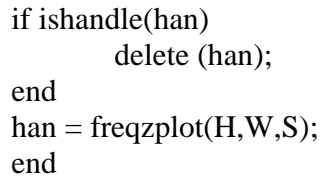




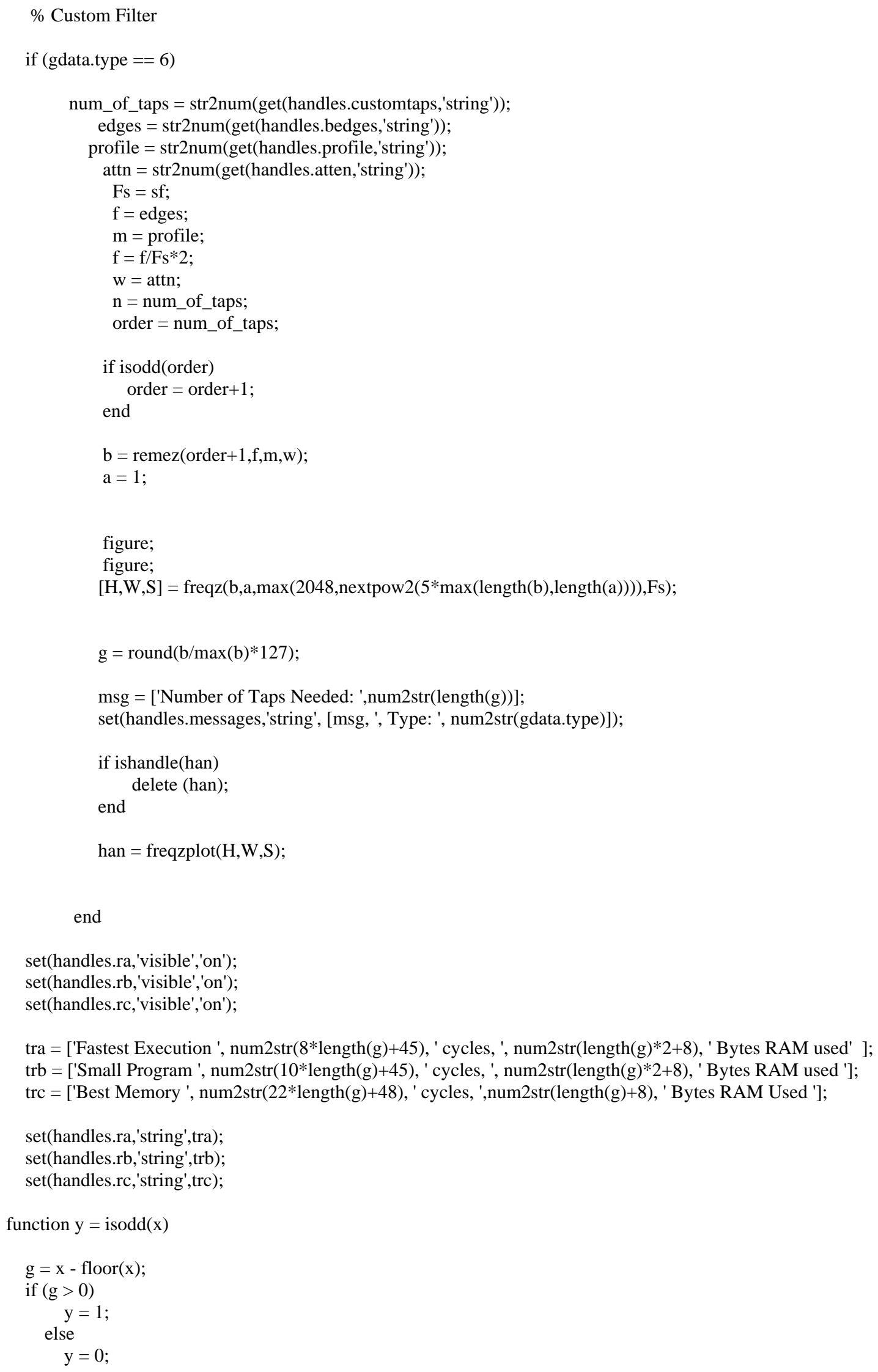


end

function custom_off()

global handles;

set(handles.cover,'visible','off');

set(handles.tprofile,'visible','off');

set(handles.profile,'visible','off');

set(handles.bedges,'visible','off');

set(handles.text12,'visible','off');

set(handles.customtaps,'visible','off');

set(handles.attnt,'visible','off');

set(handles.atten,'visible','off');

$\%$

function varargout = ra_Callback(h, eventdata, handles, varargin $)$

global imptype;

set(handles.rb, 'value', 0);

set(handles.rc, 'value', 0);

imptype $=1$;

$\%$

function varargout = rb_Callback(h, eventdata, handles, varargin)

global imptype;

set(handles.ra, 'value', 0);

set(handles.rc, 'value', 0);

imptype $=2$;

$\%$

function varargout = rc_Callback(h, eventdata, handles, varargin $)$

global imptype;

set(handles.ra, 'value', 0);

set(handles.rb, 'value', 0);

imptype $=3$;

function varargout $=$ sba_Callback(h, eventdata, handles, varargin $)$ 


\section{APPENDIX C}

CODE FOR $4^{\text {th }}$ ORDER Floating-Point LMS Filter 


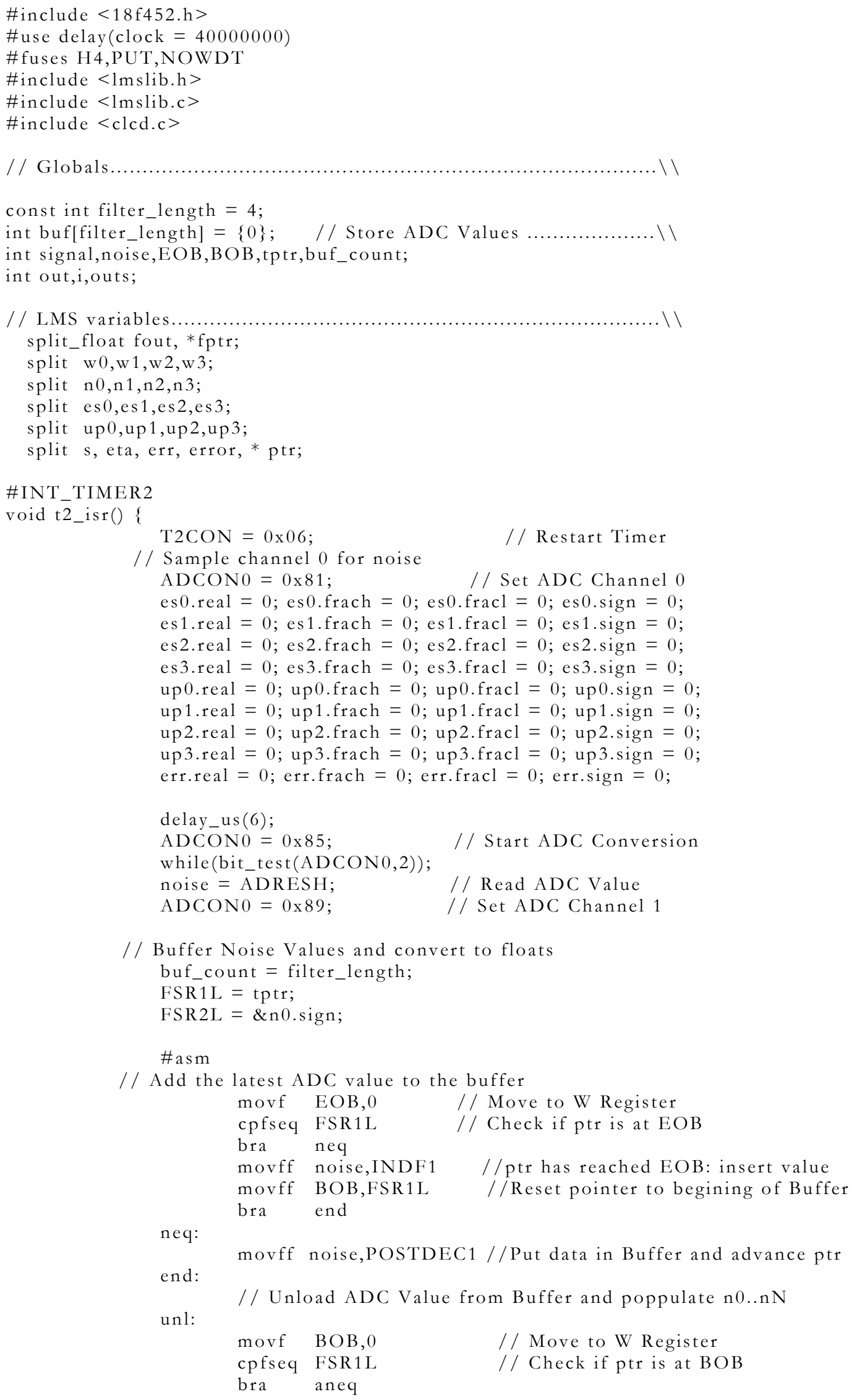




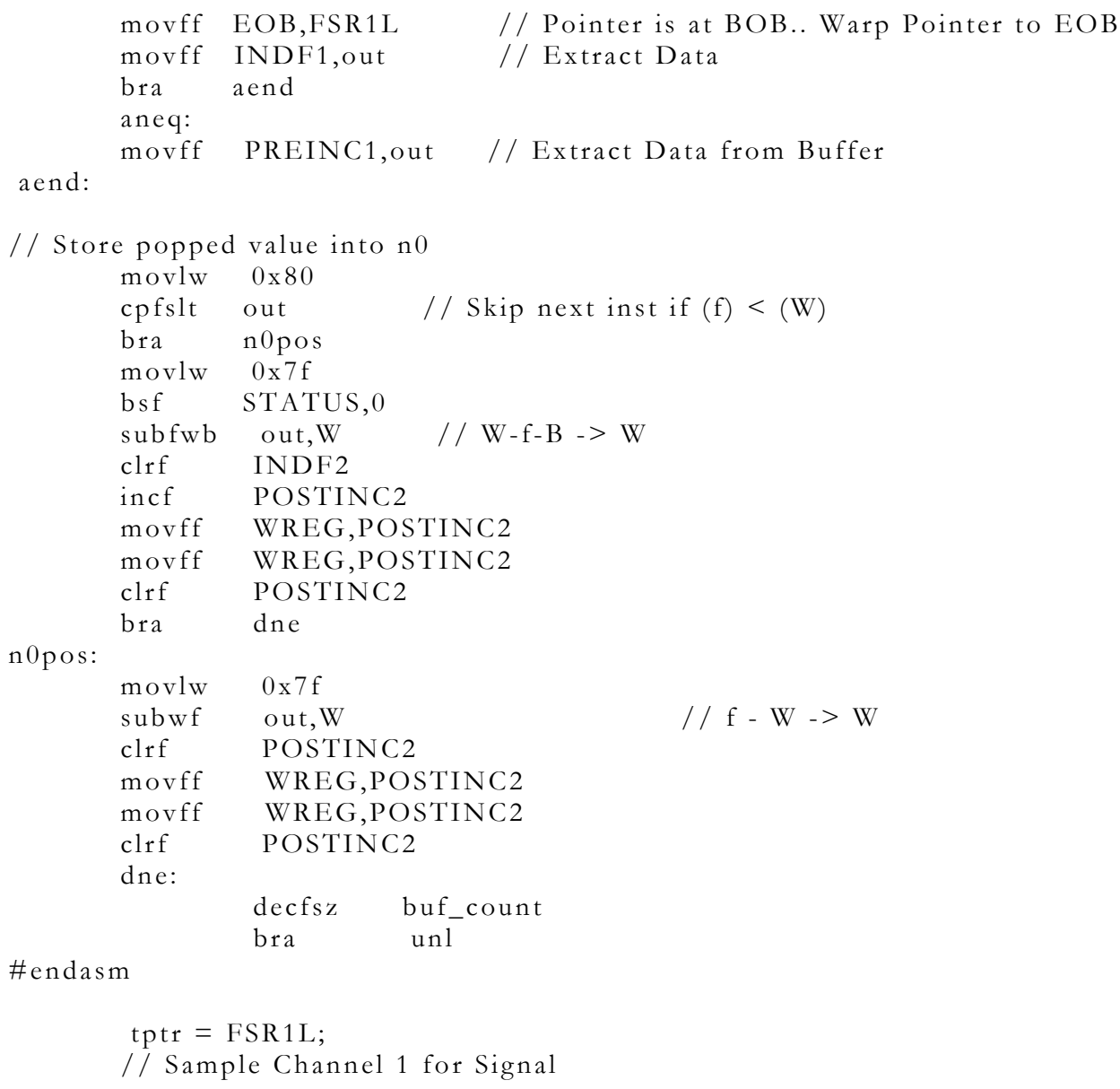




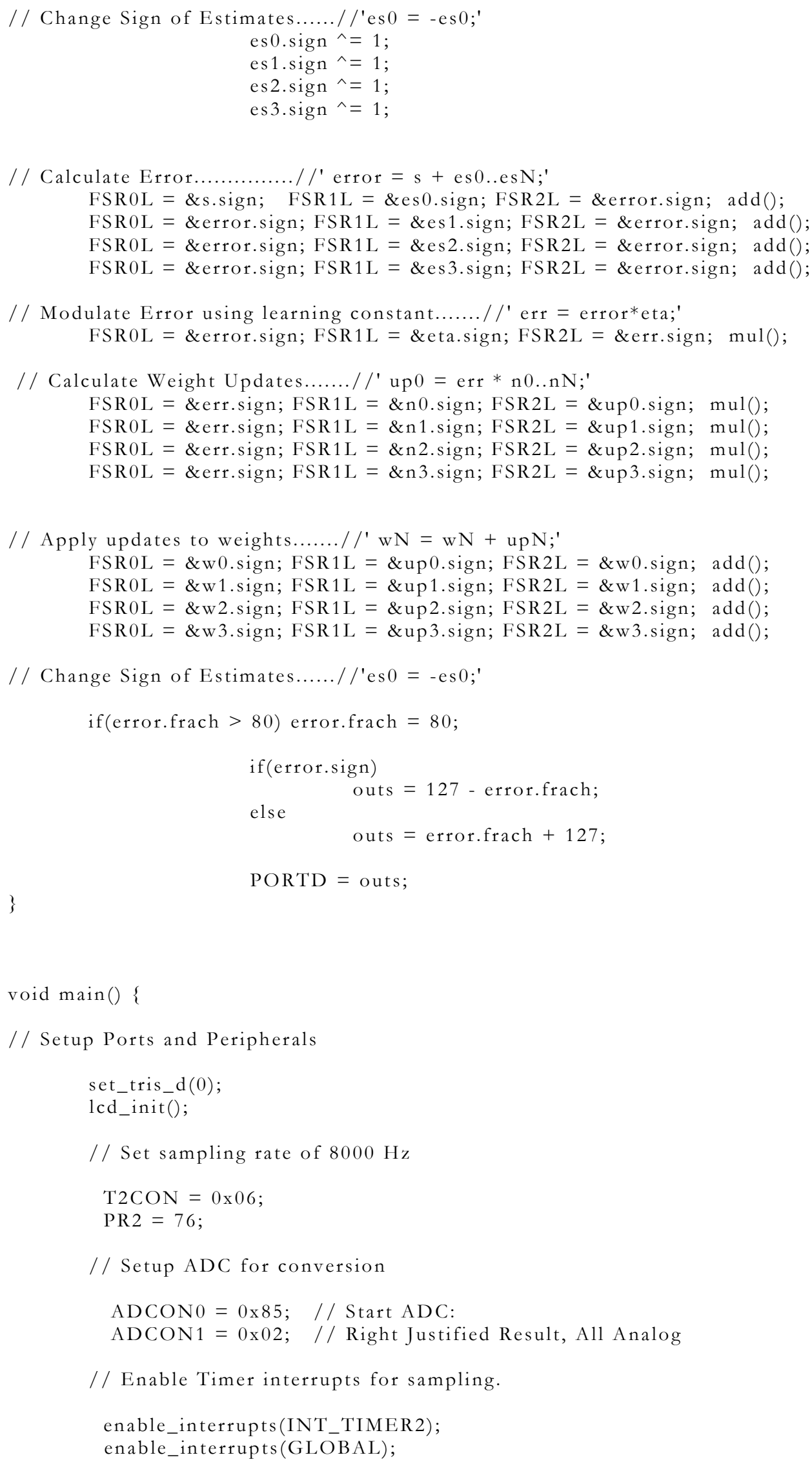


// Initialize LMS variables

$$
\begin{aligned}
& \text { ptr }=\& w 0 ; \text { fix } 8 \times 16(0.0, p t r) \\
& \text { ptr }=\& w 1 ; \text { fix } 8 \times 16(0.0, p t r) \text {; } \\
& \text { ptr }=\& w 2 \text {; fix } 8 \times 16(0.0, p t r) \text {; } \\
& \text { ptr }=\& w 3 \text {; fix } 8 \times 16(0.0, \text { ptr }) \text {; } \\
& \text { ptr }=\text { \&es } 0 ; \text { fix } 8 \times 16(0.0, p t r) \text {; } \\
& \text { ptr }=\text { \&es } 1 \text {; fix } 8 \times 16(0.0, \text { ptr }) \text {; } \\
& \text { ptr }=\text { \&es } 2 \text {; fix } 8 \times 16(0.0, p t r) \text {; } \\
& \text { ptr = \&es } 3 \text {; fix } 8 \times 16(0.0, \text { ptr }) \text {; } \\
& \text { ptr }=\text { \&up } 0 ; \text { fix } 8 \times 16(0.0, p t r) ; \\
& \text { ptr }=\text { \&up } 1 \text {; fix } 8 \times 16(0.0, p t r) \text {; } \\
& \text { ptr }=\& u p 2 ; \text { fix } 8 \times 16(0.0, p t r) \text {; } \\
& \text { ptr }=\text { \&up3; fix } 8 \times 16(0.0, p t r) \text {; } \\
& \text { ptr }=\text { \&err; fix } 8 \times 16(0.0, p t r) ; \\
& \text { ptr = \&eta; fix } 8 \times 16(0.1, p t r) \text {; } \\
& \text { ptr }=\text { \&error; fix } 8 \times 16(0.0, p t r) \text {; }
\end{aligned}
$$

// Initialize buffer pointers for LMS

$$
\begin{aligned}
& \mathrm{EOB}=\text { \&buf }[0] \\
& \text { BOB = \&buf[filter_length-1]; } \\
& \text { tptr = BOB; }
\end{aligned}
$$

while(1) \{

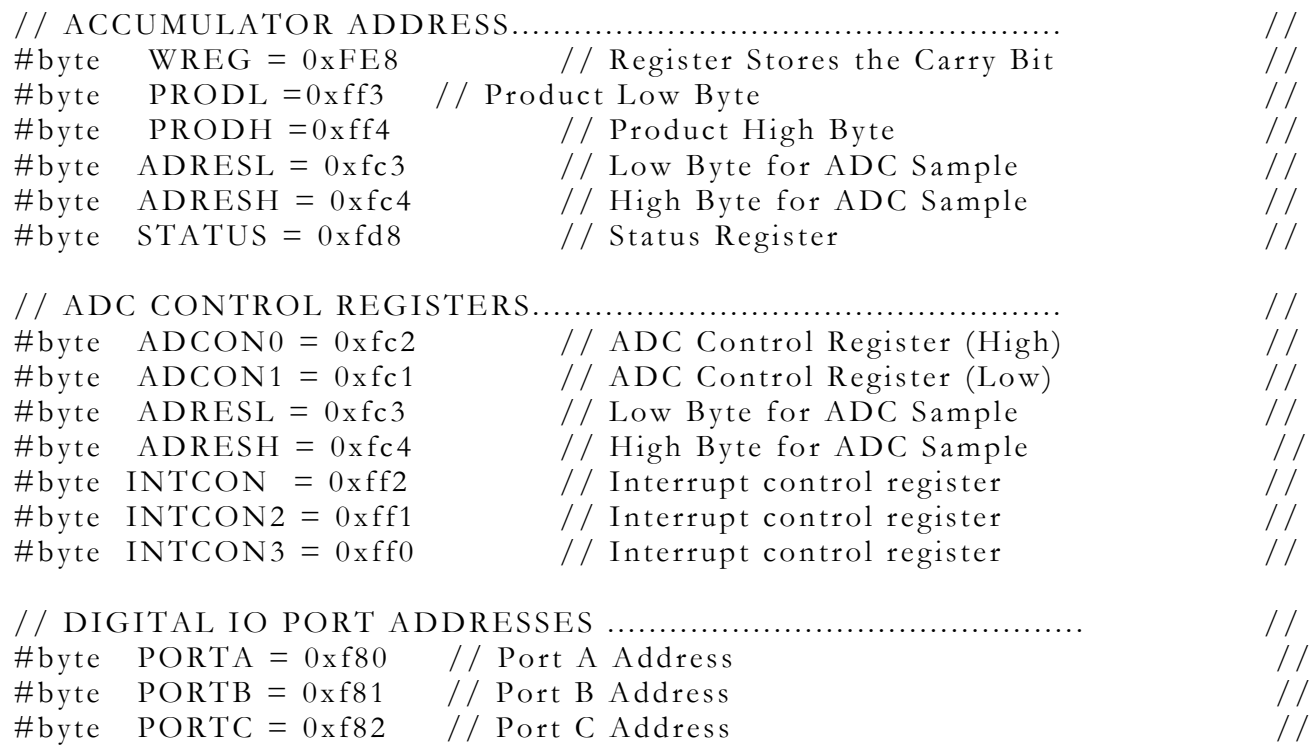




\begin{tabular}{|c|c|c|}
\hline \#byte & PORTD $=0 \times f 83$ & // Port D Address \\
\hline \#byte & PORTE $=0 \times \mathrm{xf} 84$ & // Port E Address \\
\hline \#byte & LATA $=0 \times \mathrm{f} 89$ & // Set Driection for PORTA \\
\hline \#byte & $\mathrm{LATB}=0 \mathrm{xf} 8 \mathrm{a}$ & // Set Driection for PORTB \\
\hline \#byte & $\mathrm{LATC}=0 \mathrm{xf} 8 \mathrm{~b}$ & // Set Driection for PORTC \\
\hline \#byte & LATD $=0 \mathrm{xf} 8 \mathrm{c}$ & // Set Driection for PORTD \\
\hline \#byte & $\mathrm{LATE}=0 \mathrm{xf} 8 \mathrm{~d}$ & // Set Driection for PORTE \\
\hline
\end{tabular}

// INDIRECT ADDRESSING

\#byte FSR0H = 0xfeA // Hardware File Pointer0 (High)

\#byte FSR0L = 0xfe9 // Hardware File Pointer0 (Low)

\#byte FSR1H = 0xfe2 // Hardware File Pointer1 (High)

\#byte FSR1L = 0xfe1 // Hardware File Pointer1 (Low)

\#byte FSR2H = 0xfda // Hardware File Pointer2 (High)

\#byte FSR2L = 0xfd9 // Hardware File Pointer2 (Low)

\#byte INDF0 $=0 x$ fef // Read Data Pointed by FSR0

\#byte INDF1 $=0 \times \mathrm{xfe} 7 \quad / /$ Read Data Pointed by FSR1

\#byte INDF2 $=0 \mathrm{xfdf} / /$ Read Data Pointed by FSR2

\#byte PLUSW0 $=0 \mathrm{xfeb} / /$ Add Pointed data to WREG

\#byte PLUSW1 = 0xfe3 // Add Pointed data to WREG

\#byte PLUSW2 $=0 \mathrm{xfdb} / /$ Add Pointed data to WREG

\#byte PREINC0 $=0 x$ fec $/ /$ Pre-increment pointer 0

\#byte PREINC1 $=0 x f e 4 / /$ Pre-increment pointer1

\#byte PREINC2 $=0 \mathrm{xfdc} / /$ Pre-increment pointer 2

\#byte POSTINC0 $=0 x$ fee // Post-Incerement Pointer0

\#byte POSTDEC0 $=0 \mathrm{xfed} / /$ Post-Decrement Pointer0

\#byte POSTINC1 $=0 \mathrm{xfe} 6 \quad / /$ Post-Increment Pointer1

\#byte POSTDEC1 $1=0 \mathrm{xfe} 5$ // Post-Decrement Pointer 1

\#byte POSTINC2 $=0 \mathrm{xfde} / /$ Post-Increment Pointer 2

\#byte POSTDEC2 $=0 \mathrm{xfdd} / /$ Post-Decrement Pointer2

/ / INTERRUPT REGISTERS...

\#byte INTCON $=0 x f f 2 \quad / /$ Interrupt Register 0

\#byte INTCON2 = 0xff1 // Interrupt Register2

\#byte INTCON3 = 0xff0 // Interrupt Register3

// STACK ADDRESSES

\#byte STKPTR $=0 \mathrm{xffc}$

\#byte TOSU $=0 \mathrm{xfff}$

\#byte $\quad$ TOSH $=0 x f f e$

\#byte TOSL $=0 \mathrm{xffd}$

// EEPROM ADDRESSES.

\#byte EEADR = 0xfA9 // EEPROM Register

\#byte EEDATA $=0 \times$ fa 8 // EEPROM Register

\#byte EECON2 $=0 x f a 7 \quad / /$ EEPROM Register

\#byte EECON1 = 0xfa 6 // EEPROM Register

// TIMER REGISTERS

\#byte $\mathrm{T} 2 \mathrm{CON}=0 \mathrm{xfca}$

\section{FileName: 1mslib.c}

typedef struct gtype \{

$$
\begin{aligned}
& \text { int sign; } \\
& \quad \text { int fracl; } \\
& \text { int frach; } \\
& \text { int real; } \\
& \text { \} split; }
\end{aligned}
$$




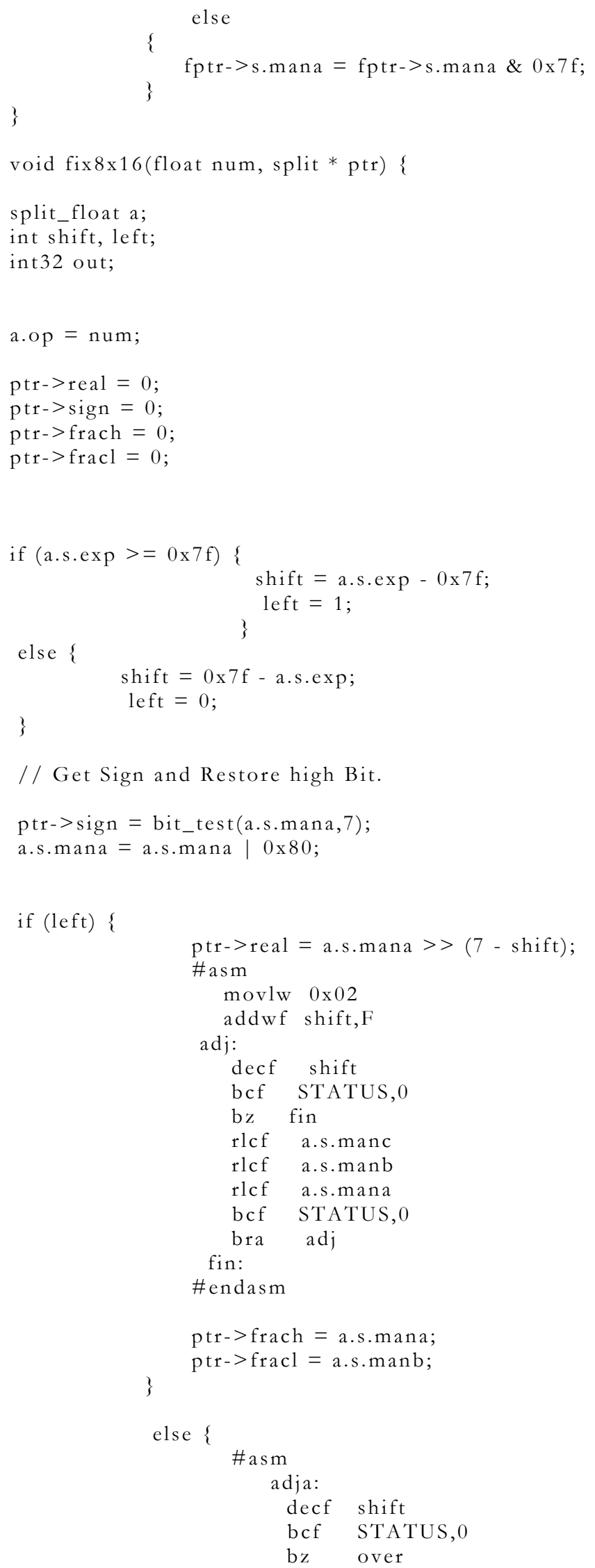




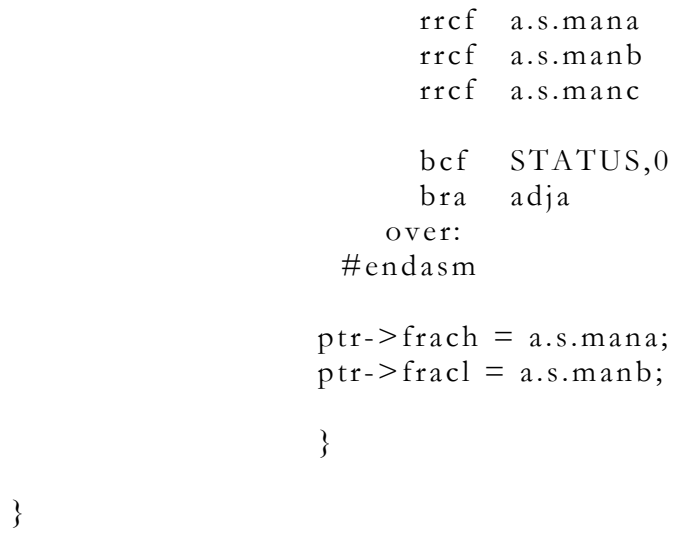




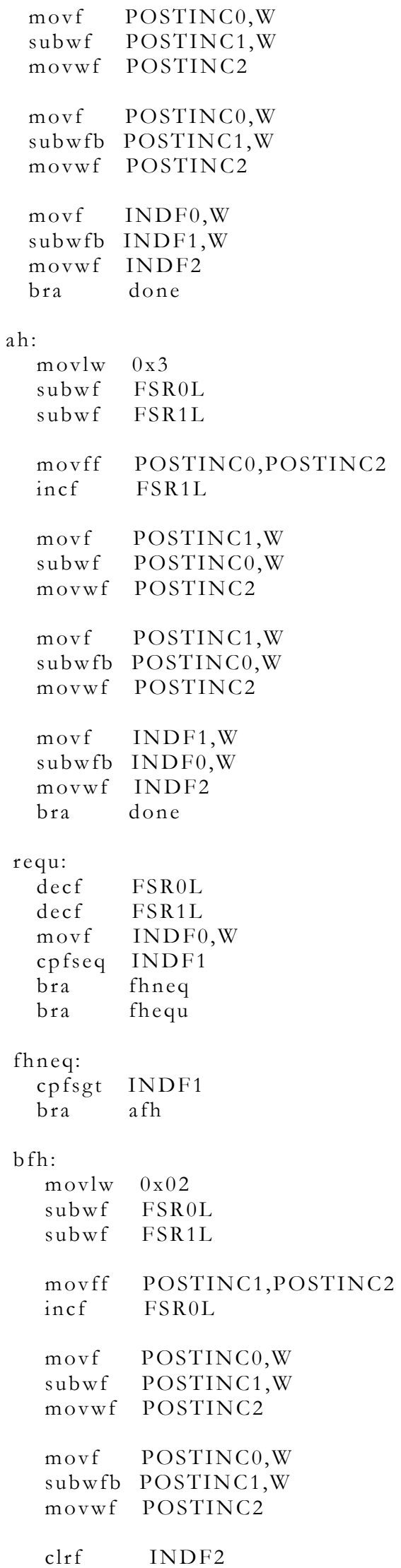




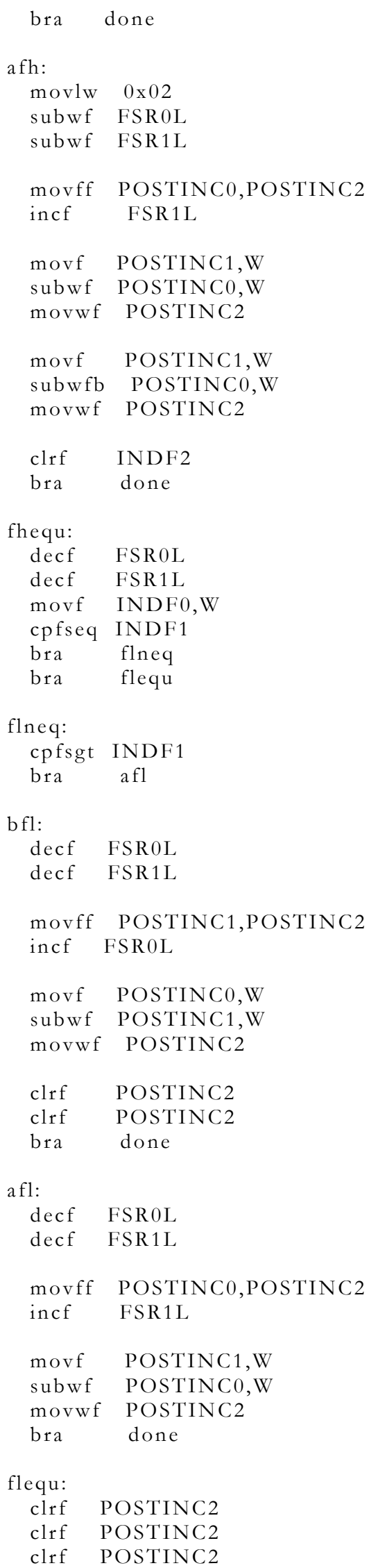




\section{APPENDIX D}

C-Code for the Clock Signal to the Switched Cap filter 
// The following code generates a $50000 \mathrm{~Hz}$ Clock signal of $55555 \mathrm{~Hz}$ // Allowing the Switched capasitor MAX 297 to have a cutoff of //1 KHz.

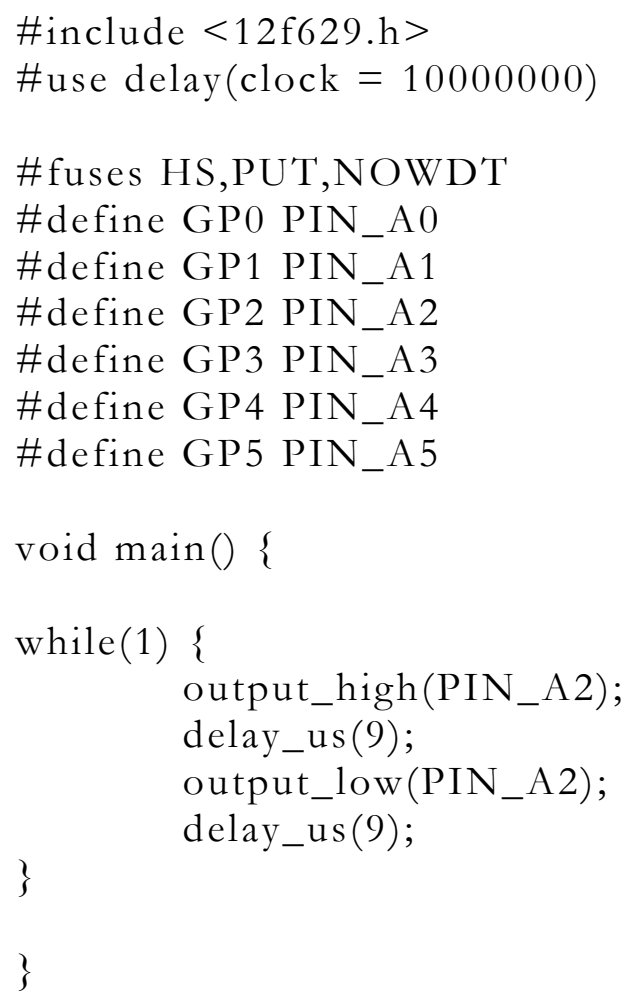




\section{APPENDIX E}

C-Code for the PORTC HD44780 LCD DEVICE 


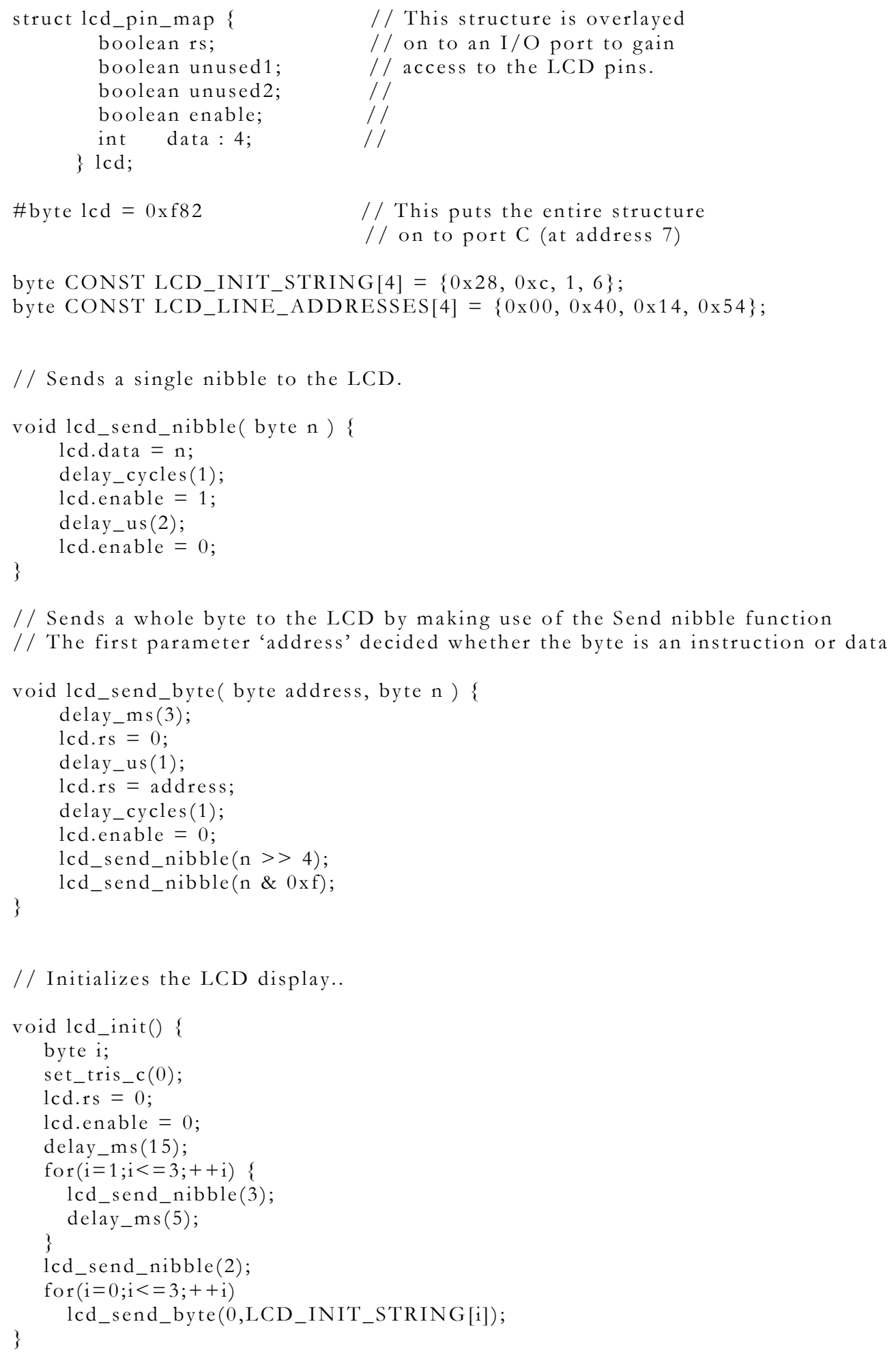


// Sets the cursor on the screen where the character is to be printed.

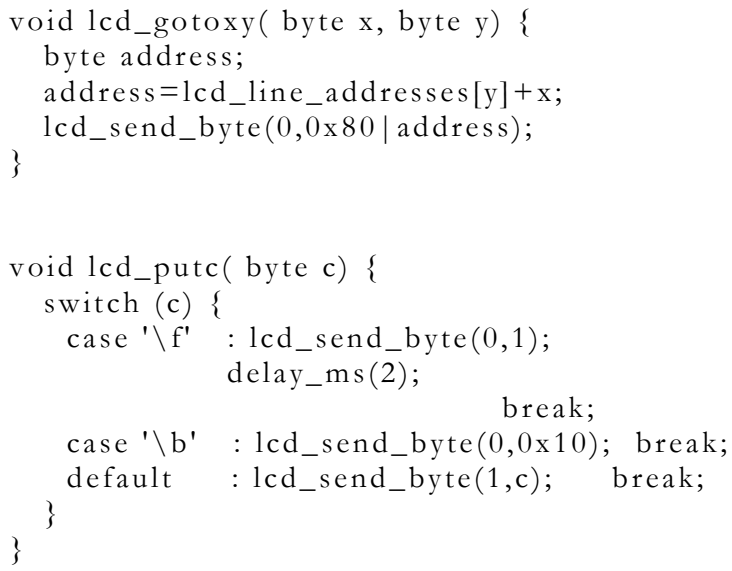




\section{VITA}

I was born in Lucknow, India and I spent most of my childhood in New Delhi. I received my undergraduate in Electrical Engineering from West Virginia University and continued on to finish my masters degree

from here as well. During my graduate years, I have held both GTA and GRA positions and spend my time doing either research or teaching. My research interests, inherited from my teacher Dr. Klinkhachorn, are Neural Networks, Fuzzy Logic, Digital Filter design and implementation, and embedded control. 